\title{
A CHECKLIST OF THE FLOWERING PLANTS OF KATERNIAGHAT WiLDLIFE SANCtUARY, UtTAR PRADESh, INDIA
}

\section{Anoop Kumar ${ }^{1}$, Omesh Bajpai ${ }^{2}$, Ashish Kumar Mishra ${ }^{3}$, Nayan Sahu ${ }^{4}$, Soumit Kumar Behera ${ }^{5}$, Surendra Singh Bargali ${ }^{6} \&$ Lal Babu Chaudhary ${ }^{7}$}

1,2,3,4,5,7 Plant Diversity, Systematics and Herbarium Division, CSIR-National Botanical Research Institute, Lucknow, Uttar Pradesh 226001, India

${ }^{6}$ Department of Botany, Kumaun University, Nainital, Uttarakhand, India

${ }^{1}$ srivastava.a.k.80@gmail.com, ${ }^{2}$ omeshbajpai@gmail.com, ${ }^{3}$ ashishmishramlg@gmail.com , ${ }^{4}$ sahunayan60@gmail.com,

${ }^{5}$ soumitkbehera@gmail.com, ${ }^{6}$ surendrakiran@rediffmail.com, ${ }^{7} \mathrm{dr}$ _lbchaudhary@rediffmail.com (corresponding author)

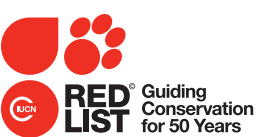

\begin{abstract}
Katerniaghat Wildlife Sanctuary, a tropical moist deciduous forest along the Indo-Nepal boarder comprises of 778 species of angiosperms, out of which 613 species are dicots under 386 genera and 91 families and 165 species are monocots under 103 genera and 23 families. It contains 82 species that are in cultivation and/or growing as alien invasives. The species include 149 trees, 81 shrubs, 445 herbs and 103 climbers. Fabaceae with 100 species and Poaceae with 65 species occupy the first position in dicots and monocots, respectively. Cyperus with 14 species has been found to be the largest genus represented while 355 genera are represented by solitary species. The present study enumerates all species of flowering plants occurring in the sanctuary area with their correct name along with first citation and some important references pertaining to the flora of the study area. Important synonyms have also been provided. For majority of species the representative voucher specimens have also been supplied. The paper also briefly deals with the vegetation types of the area. The outcome of the work is based on extensive field survey of the area conducted during 2008-2011, study of literature and examination of specimens of earlier collections housed at BSA, BSIP, CDRI and LWG.
\end{abstract}

Keywords: Angiosperms, checklist, flora, Katerniaghat Wildlife Sanctuary, nomenclature.

DOI: http://dx.doi.org/10.11609/JoTT.03257.7309-408

Editor: Ravi Prasad Rao, Sri Krishnadevaraya University, Anantapur, India.

Date of publication: 19 June 2015 (online \& print)

Manuscript details: Ms \# o3257 | Received 07 March 2013 | Final received 30 January 2014 | Finally accepted 05 March 2015

Citation: Kumar, A., O. Bajpai, A.K. Mishra, N. Sahu, S.K. Behera, S.S. Bargali \& L.B. Chaudhary (2015). A checklist of the flowering plants of Katerniaghat Wildlife Sanctuary, Uttar Pradesh, India. Journal of Threatened Taxa 7(7): 7309-7408; http://dx.doi.org/10.11609/JoTT.03257.7309-408

Copyright: (c) Kumar et al. 2015. Creative Commons Attribution 4.0 International License. JoTT allows unrestricted use of this article in any medium, reproduction and distribution by providing adequate credit to the authors and the source of publication.

Funding: CSIR, New Delhi for financial support under NWP-020.

Competing Interest: The authors declare no competing interests.

Author Details: Mr. Anoop Kumar works on angiosperm taxonomy since last four years on different aspects including flora of KWS; Mr. Omesh Bajpai works on taxonomy and phenology of tree flora of terai region including KWS since last four years; Mr. Ashish K. Mishra works on plant ecology and phytosociology of KWS since last four years; Mr. Nayan Sahu works on phytosociology and ecophysiology of KWS since last four years; Mr. Soumit K. Behera works on plant ecology since last 10 years including KWS; Prof. Surendra Singh Bargali works on different aspects of ecology and guides PhD students; Dr. Lal Babu Chaudhary works on angiosperm taxonomy since last 24 years including tree flora of Uttar Pradesh and KWS.

Author Contribution: AK - survey, collection, identification and writing manuscript; OB - survey, collection, identification and phenological data; AKM - survey, collection, provided ecological information; NS - survey, collection, provided ecological information; SKB - survey, collection, provided ecological information and management of the project; SSB - provided guidelines and discussion on the subject; LBC - survey, collection, identification, structure of manuscript and overall management of the work.

Acknowledgements: The authors are sincerely grateful to Dr. C.S. Nautiyal, Director, CSIR - National Botanical Reseach Institute, Lucknow for facilities and encouragement and CSIR, New Delhi for financial support under NWP-020. Thanks are due to the in-charge of herbaria mentioned in the work for granting permission for herbarium consultation. The DFO and all staff of Katerniaghat Wildlife Sanctuary are greatefully acknowledged for their hospitality and assistance provided during the field work. We are also thankful to Ms. Shriti Dutta for her help in computer work.

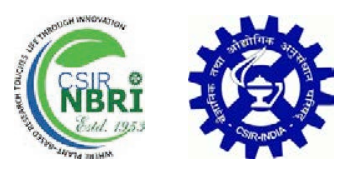




\section{INTRODUCTION}

After the Rio de Janeiro Convention on Biological Diversity in 1992, exploration of biodiversity becomes more important than ever to know the cause of rapid depletion of biodiversity, their conservation management and to study the effects of climate change. In addition to this, the updated list of plants of a particular region helps to prevent bio-piracy and safeguard traditional knowledge. In this perspective, the Katerniaghat Wildlife Sanctuary was selected for its proper documentation and assessment.

Uttar Pradesh is one of the largest provinces of India occupying an area of about $2,40,928 \mathrm{~km}^{2}$, however, its recorded forest area is about $16,583 \mathrm{~km}^{2}$ which is only ca. $7 \%$ of its geographical area because its maximum area is occupied by agriculture lands and human populations (Anonymous 2011). For safeguarding biodiversity, 23 sanctuaries and one national park have been declared in the province (Anonymous 2011; Srivastava 2004, 2011). Katerniaghat, declared as wildlife sanctuary in May 1976 comprises about $551.64 \mathrm{~km}^{2}$ area (ca. $45 \mathrm{~km}$ long and $12 \mathrm{~km}$ wide) which includes Katerniaghat, Nishangarah, Dharmapur, Murthia, Kakraha and Motipur ranges (Fig. 1). The sanctuary is one of the most diverse tropical moist deciduous forests in the upper Gangetic plain of Bahraich District in the Terai region along the southern border of Nepal between $27^{\circ} 41^{\prime}-27^{0} 56^{\prime} \mathrm{N}$ and $81^{\circ} 48^{\prime}-81^{\circ} 56^{\prime} \mathrm{E}$ with elevation ranges from $116-165 \mathrm{~m}$. The forest division is intersected by an approximately $40 \mathrm{~km}$ long metallic road from east to west parallel to the railway track which also passes through the forest. The river Girwa flows adjoining to the sanctuary area which meets with Kauriala River at Girijapuri irrigation barrage adjacent to Katerniaghat range. The forest area is also surrounded by several villages from all sides. This imposes anthropogenic pressure on the forest resulting in rapid depletion of plant diversity. Hence, in the present study the whole sanctuary area has been selected to document its entire native plant diversity of angiosperm flora, and cultivated and invasive species.

\section{VEGETATION AND CLIMATE}

On the basis of floristic composition the entire sanctuary consists of chiefly four types of forest communities like sal mixed forest, swamp forest, miscellaneous forest and grassland, and an artificial type -- teak plantation. Sal mixed forest, which covers the major portion of the sanctuary, is easily recognized by their large trees which constitute the top most canopy of the forest. Although, in Sal forest, Shorea robusta Gaertn. f. (Sal) is the main dominant species, Terminalia elliptica Willd., Diospyros exculpta Buch.-Ham., Haldina cordifolia (Roxb.) Ridsdale, Mitragyna parvifolia (Roxb.) Korth., Syzygium cumini (L.) Skeels, Hymenodictyon orixense (Roxb.) Mabberley, Lagerstroemia parviflora Roxb., and Ehretia laevis Roxb. are also frequently found. The teak plantation has some ground vegetation dominated by climbers like Tiliacora acuminata (Lam.) Miers., and some tree species such as Mallotus philippensis (Lam.) Muell.-Arg., Ficus hispida L.f. and F. squamosa Roxb. are represented here occasionally. The low land forest community is chiefly dominated by Syzygium cumini (L.) Skeels, Ficus hispida L.f., Barringtonia acutangula (L.) Gaertn., Mallotus nudiflorus (L.) Kulju \& Welzen, Bridelia stipularis (L.) Bl., Glycosmis pentaphylla (Retz.) DC. and Calamus tenuis Roxb. etc. Mixed forest occupies a large stretch of the sanctuary area chiefly dominated by Syzygium cumini (L.) Skeels, Haldina cordifolia (Roxb.) Ridsdale, Madhuca longifolia (L.) Macbr. var. latifolia (Roxb.) Chev., Schleichera oleosa (Lour.) Merr., Ficus rumphii Blume, F. hispida L. f., Haldina cordifolia (Roxb.) Ridsdale, Mitragyna parvifolia (Roxb.) Korth., Aegle marmelos (L.) Correa, Cassia fistula L., Bauhinia spp., Miliusa tomentosa (Roxb.) Sinclair, Bridelia retusa (L.) A. Juss., Ehretia laevis Roxb., Buchanania cochinchinensis (Lour.) Almeida, Diospyros exculpta Buch.-Ham., Bombax ceiba L., Dalbergia sissoo Roxb. ex DC. among others. The savanna-grassland community mainly found in core zone of the sanctuary area is mainly represented by the members of Poaceae like Desmostachya bipinnata (L.) Stapf., Dendrocalamus strictus (Roxb.) Nees, and Saccharum spontaneum L. along with some trees of Grewia tillifolia Vahl, Sterculia villosa Roxb. ex Sm., Ceriscoides turgida (Roxb.) Tirveng. and Hymenodictyon orixense (Roxb.) Mabberley.

Vertically, the forest vegetation may be divided into upper, middle and lower strata and ground vegetation. The upper stratum constitutes the canopy of large trees like Shorea robusta Gaertn. f., Tectona grandis L.f., Terminalia elliptica Willd., Madhuca longifolia (L.) Macbr. var. latifolia (Roxb.) Chev., Ficus benghalensis L., Ficus racemosa L., Bombax ceiba L., Sterculia villosa Roxb. ex Sm. and Lannea coromandelica (Houtt.) Merr. The midddle stratum is represented by Hymenodictyon orixense (Roxb.) Mabberley, Syzygium cumini (L.) Skeels, Ehretia laevis Roxb., Lagerstroemia parviflora Roxb., Diospyros exculpta Buch.-Ham., Mallotus philippensis (Lam.) Muell.-Arg., M. nudiflorus (L.) Kulju \& Welzen, Ficus hispida L.f. and Streblus asper Lour. The lower 


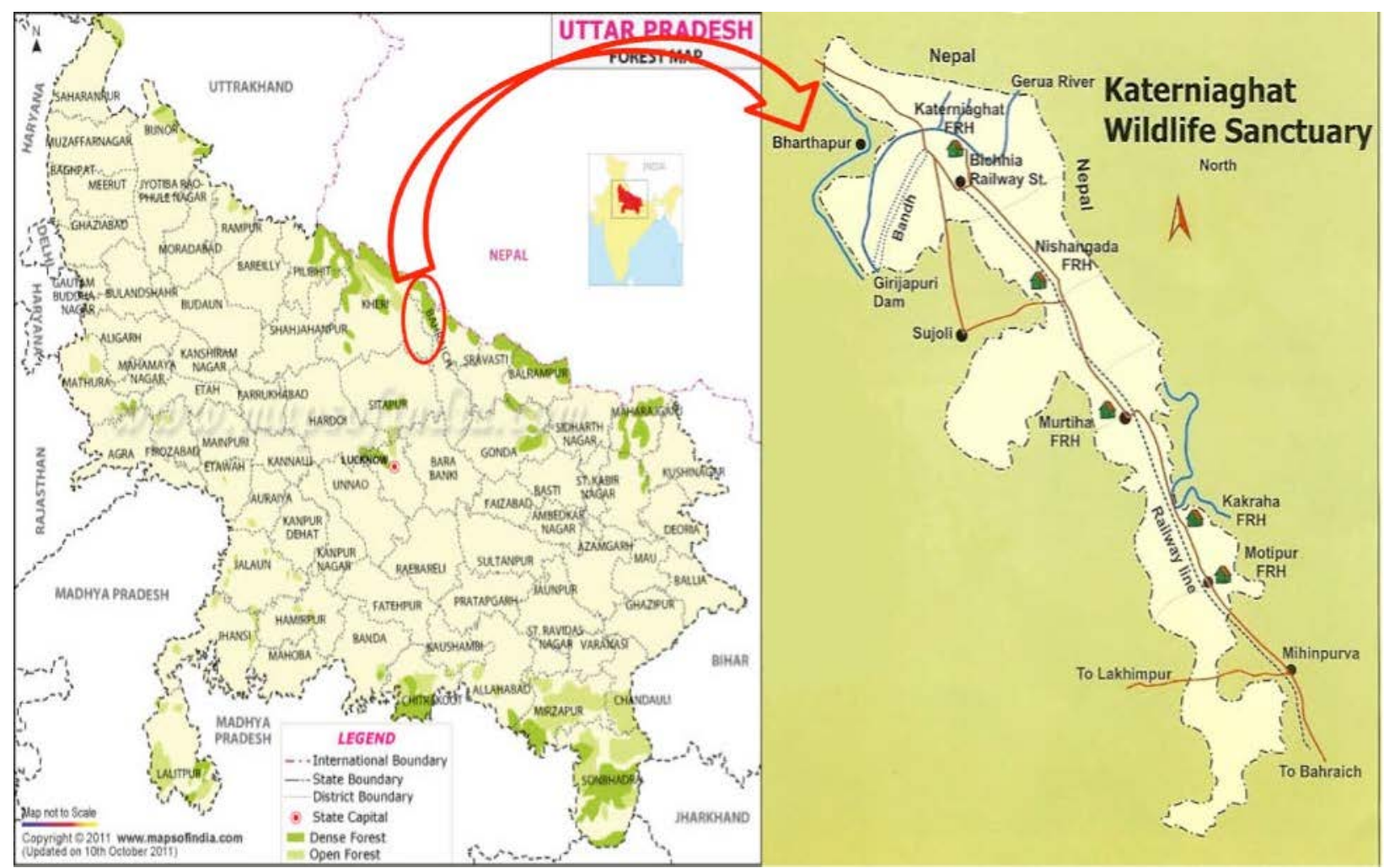

Figure 1. Location of Katerniaghat Wildlife Sanctuary in Uttar Pradesh. Source: Uttar Pradesh map from http://www.mapsofindia.com/maps/ uttarpradesh/uttarpradesh-forest-map.htm and KWS from: http://dudhwatigerreserve.com/images/dudhwamap.jpg

stratum where the minimal light is available consists of Bergera koenigii L., Glycosmis pentaphylla (Retz.) DC., Clerodendrum viscosum Vent., Grewia hirsuta Vahl and Lantana camara L. The ground vegetation is mainly covered by annual and perennial herbs like Polygala arvensis Willd., Primula umbellata (Lour.) Bentv., Anagallis arvensis L., Fumaria indica (Hausskn.) Pugsley, Salvia plebia R. Br. and Melilotus indicus (L.) All. Tiliacora acuminata (Lam.) Miers, Ichnocarpus frutescens (L.) Ait., Smilax ovalifolia Roxb. ex D. Don, Hippocratea arborea Roxb., Bauhinia vahlii Wight. \& Arn. and Bridelia stipularis (L.) BI. are some of the dominant climbers and creepers in the forest.

The climate of the area is monsoonal. The area witnesses three distinct seasonal variations: winter (November-February), summer (April-June) and warmrainy (July-September). The mean minimum and maximum temperature varied from $8-22{ }^{\circ} \mathrm{C}$ in January and to $27-40{ }^{\circ} \mathrm{C}$ in May-June. The annual rainfall varies from 36-142 $\mathrm{mm}$ in winter, 34-662 $\mathrm{mm}$ in summer and 1294-1689 mm in warm-rainy seasons (Bajpai et al. 2012). Detailed studies on ecological parmeters and micro-climatic variables like photosynthetically active radiation (PAR), ambient $\mathrm{CO}_{2}$, air temperature and soil surface temperature in Katerniaghat Wildlife Sanctuary have already been done recently by Behera et al. (2012).

\section{LITERATURE REVIEW}

The information on the flora of Katerniaghat Wildlife Sanctuary are available in scattered form in different publications (Panigrahi et al. 1969; Saini 2005; Pandey \& Mall 2009; Pandey et al. 2008, 2009; Tripathi \& Singh 2009; Behera et al. 2012). Recently Maliya \& Datt (2010) and Maliya (2011, 2012, 2012a) have prepared a preliminary list of plants occurring in the sanctuary area which include only about 433 species. With more field trips conducted and different herbaria consulted in the present study, a large numer of species have been added. Kumar et al. (2012) have currently produced a very illustrative account of 11 species of Ficus available in Katerniaghat Wildlife Sanctuary. 


\section{MATERIALS AND METHODS}

From 2008 to 2011 Katerniaghat Wildlife Sanctuaty was thoroughly explored in different seasons to collect and document the plant diversity. Plants were randomly collected from all kinds of habitats and vegetations following standard herbarium techniques (Lawrence 1951; Jain \& Rao 1977). All collected plant materials have been deposited at LWG in the form of herbarium specimens for future record. All species have been correctly identified with the help of flora, revision and published work and compared with earlier authentic collections housed at BSA, BSIP, CDRI and LWG. The correct nomenclature has been provided after consulting large number of recent regional and national floras, literature and different websites like GRIN, IPNI, ILDIS, The Plant List, Wikipedia, Tropicos, etc. In the present study the species have been assigned to the family and genera as per recent taxonomic treatments. The families, the genera under each family and species under each genus have been arranged alphabetically for the sake of convenience. Each species has been provided with correct name along with first citation and some important references pertaining to the flora of the study area. Important synonyms have also been provided. For majority of species the representative voucher specimens have also been included. Some species have been listed only based on previous reports as their specimens were not observed either in any herbarium or in the field. Cultivated species included in the list have been marked with an '*' and also indicated in the habit of the plant. Escapees and alien invasives have been marked with '**'. Colour photographs and images of herbarium specimens have been provided for some native species.

\section{RESULT}

After the critical study of our own collections, survey of literature and examination of previous collections housed at BSA, BSIP, CDRI and LWG, 778 species of native and introduced plants have been included here out of which 613 species under 386 genera and 91 families belong to dicots and 165 species under 103 genera and 23 families to monocots (Fig. 2). These species include 149 trees belonging to 105 genera and 41 families, 81 shrubs belonging to 54 genera and 23 families, 445 herbs belonging to 287 genera and 73 families and 103 climbers belonging to 77 genera and 31 families (Fig. 3). Among different families in dicots,
Fabaceae occupies the highest position with 100 species and 46 genera followed by Asteraceae ( 51 spp. and 40 genera), Malvaceae (36 spp. and 12 genera), Lamiaceae (29 spp. and 19 genera) and Acanthaceae (26 spp. and 14 genera) and so on (Fig. 4) while in monocots the largest family is Poaceae having 65 species under 44 genera followed by Cyperaceae (34 spp. and 10 genera), Orchidaceae ( 9 spp. and 7 genera), Zingiberaceae (6 spp. and 5 genera), Asparagaceae ( 6 spp. and 4 genera) and Hydrocharitaceae ( $6 \mathrm{spp}$. and 6 genera) and so on (Fig. 5). In Fig. 6, the 20 dominant families of both dicots and monocots have been included based on number of species. Cyperus L. (14 spp.) has been found as the largest genus among all groups followed by Ficus L. (13 spp.), Grewia L. (10 spp.), Crotalaria L. (9 spp.), Solanum L. (9 spp.), and Ipomoea L. (8 spp.) (Fig. 7). On the other hand, about 48 families and 355 genera are represented by a solitary genus and species respectively. Aegle marmelos (L.) Correa, Diospyros exsculpta Buch.-

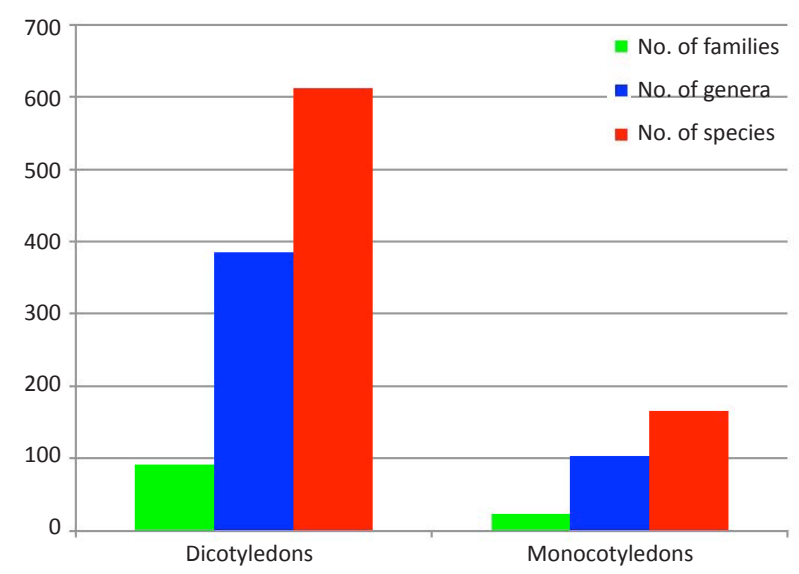

Figure 2. Floristic analysis of plants of Katerniaghat Wildlife Sanctuary

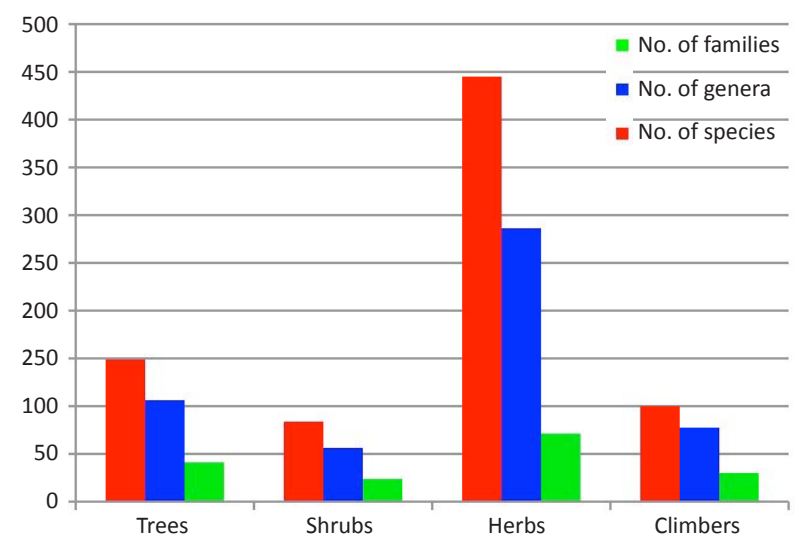

Figure 3. Different life forms 


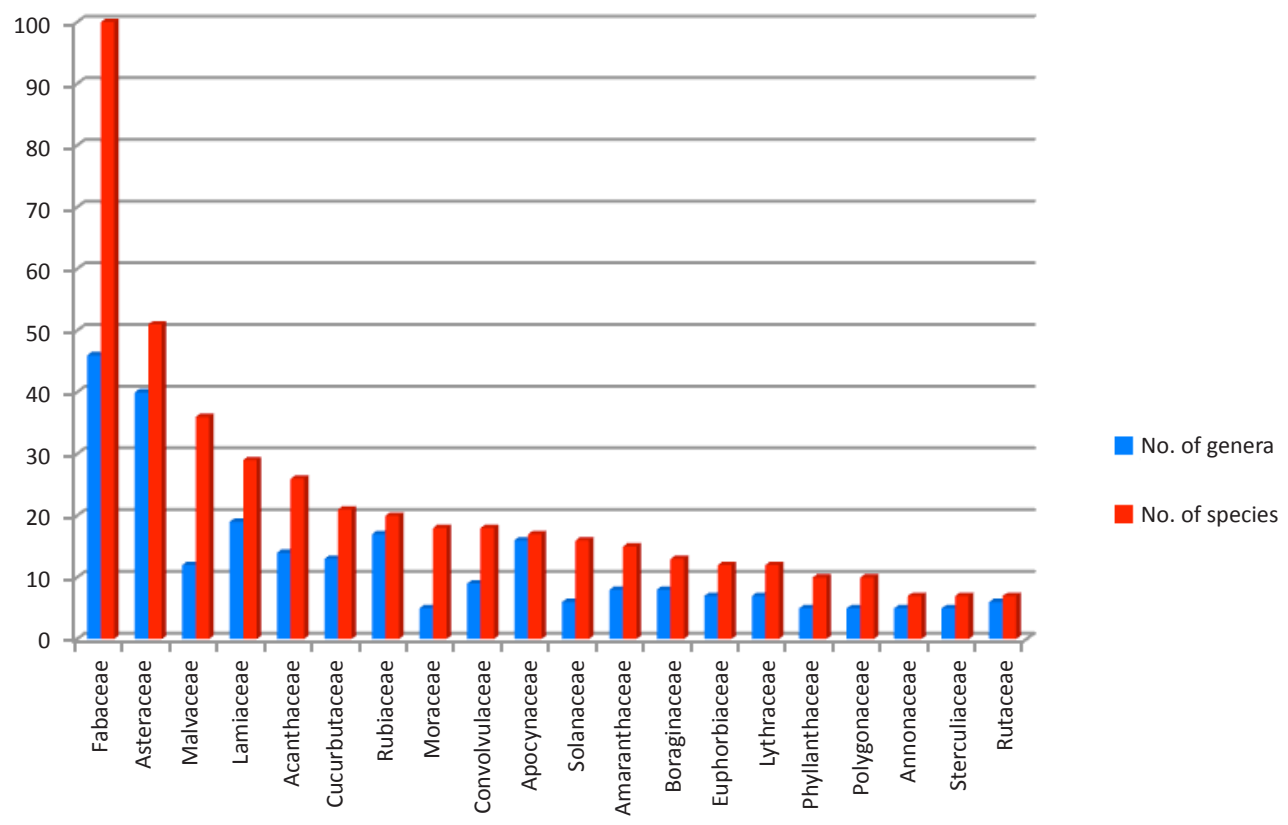

Figure 4. Largest families in dicots

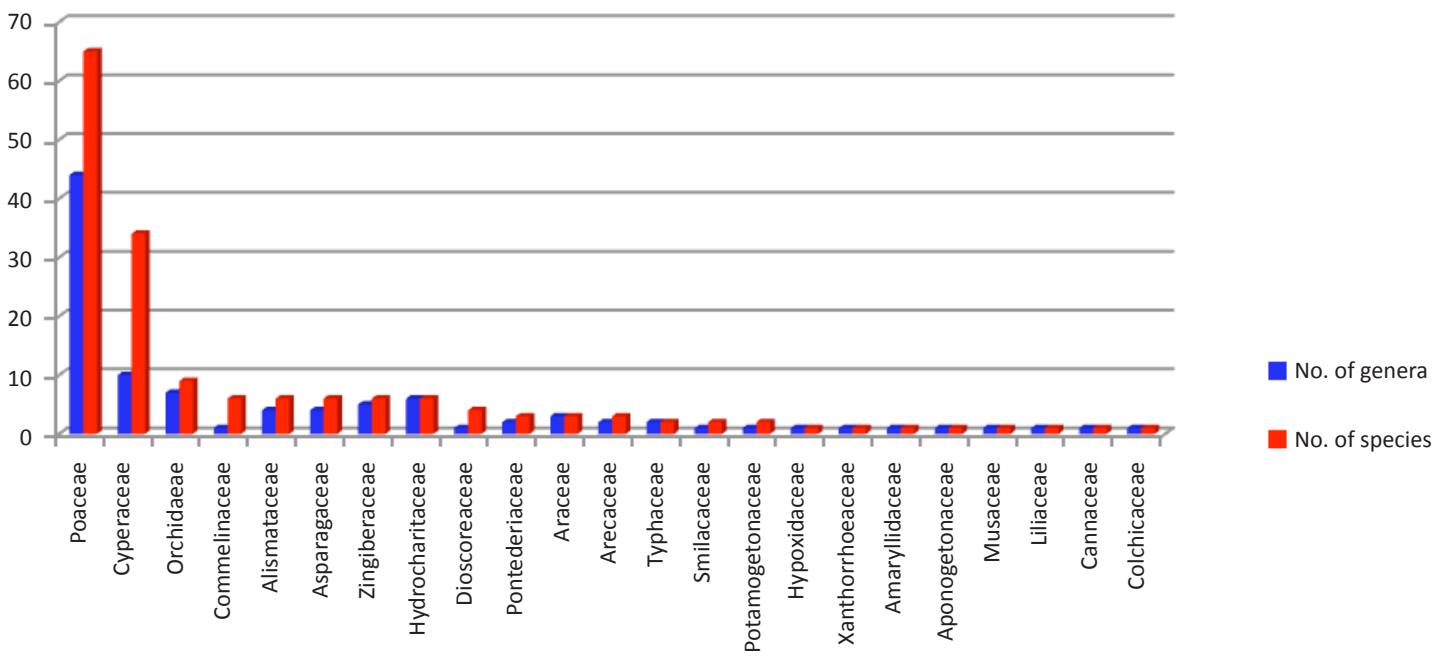

Figure 5. Largest families in monocots

Ham., Ehretia laevis Roxb., Ficus hispida L. f, Madhuca longifolia (L.) Macbr. var. Iatifolia (Roxb.) Chev., Mallotus philippensis (Lam.) Muell.-Arg., M. nudiflorus (L.) Kulju \& Welzen, Shorea robusta Gaertn. f., Syzygium cumini (L.) Skeels, Tectona grandis L. f., Terminalia elliptica Willd. etc. are some of the dominant tree species in the forest. The species which are cultivated and invasives in the forest area constitute about 10.5\% (approximate 82 species) of the total flora. Since the forest area is surrounded by agricultural fields and many villages, many ornamental and cultivated species are seen in the forest. In addition, the forest department also plant non-native ornamentals near rest houses and along roadsides for beautification of the sanctuary.

\section{DICOTYLEDONS}

\section{ACANTHACEAE}

Andrographis paniculata (Burm. f.) Wall. ex Nees in Wall., PI. Asiat. Rar. 3: 116. 1832; Saini, J. Econ. Taxon. Bot. 29 (4): 846. 2005; Maliya \& Datt, J. Econ. Taxon. Bot. 34 (1): 59. 2010.

Justicia paniculata Burm f., FI. Ind. 9. 1768.

Habit: Annual herb. 


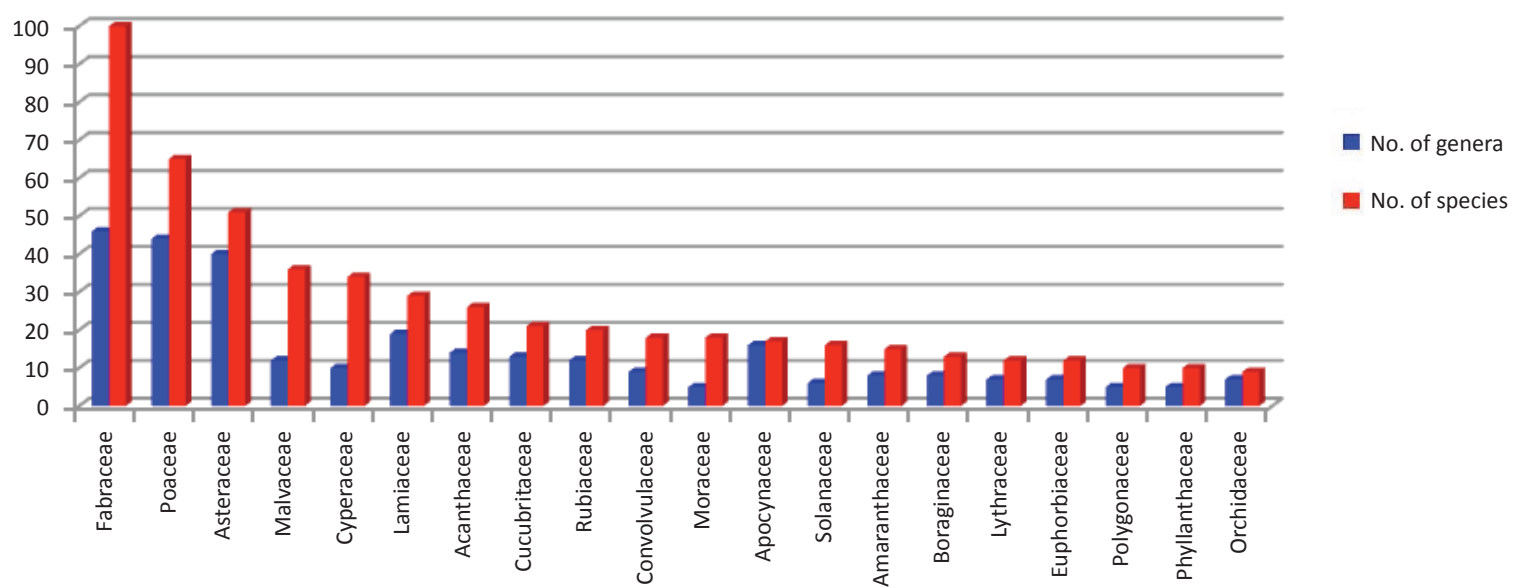

Figure 6. Largest families with their genera and species in dicots and monocots
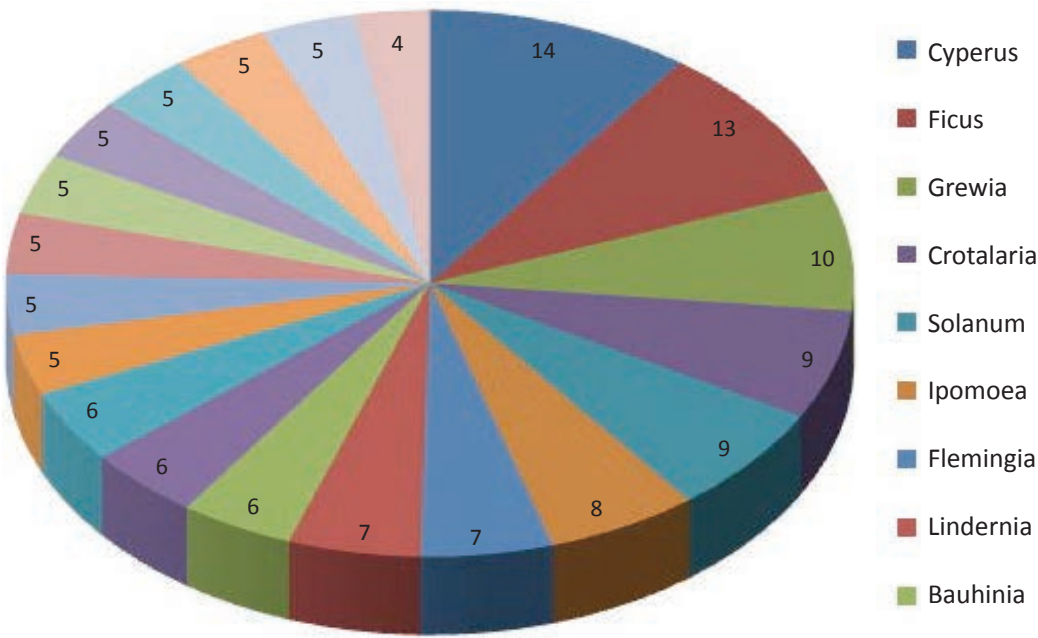

Figure 7. Largest genera

Specimens examined: Not observed. Record based on Saini (2005) and Maliya \& Datt (2010).

Barleria cristata L., Sp. PI. 636. 1753; Clarke in Hook. f., Fl. Brit. India 4: 488. 1884; Duthie, Fl. Upp. Gang. Plain 2: 70. repr. ed. 1960; Panigrahi et al., Bull. Bot. Surv. India 11 (1 \& 2): 107. 1969; Maliya \& Datt, J. Econ. Taxon. Bot. 34 (1): 59. 2010.

Habit: Undershrub.

Specimens examined: 13448 (LWG), 26.v.1954, Motipur forest, coll. Hira Lal; 224568 (LWG), 10.ii.2002, Murtiha range, coll. S.D. Maliya; 224642 (LWG), 12.ii.2002, Nishangara forest range, coll. S.D. Maliya.

Dicliptera bupleuroides Nees in Wall., Pl. Asiat. Rar. 3: 111. 1832; Maliya \& Datt, J. Econ. Taxon. Bot. 34 (1): 59. 2010.
Habit: Herb.

Specimens examined: Not observed. Record based on Maliya \& Datt (2010).

Dicliptera chinensis (L.) Juss., Ann. Mus. Hist. Nat. 9: 268. 1807.

Justicia chinensis L., Sp. PI. 16. 1753. Dicliptera roxburghiana Nees in Wall., PI. Asiat. Rar. 3: 111. 1832; Maliya \& Datt, J. Econ. Taxon. Bot. 34 (1): 59. 2010.

Habit: Herb.

Specimens examined: 224568 (LWG), 10.ii.2002, Murtiha range, coll. S.D. Maliya; 224642 (LWG), 12.ii.2002, Nishangara range, coll. S.D. Maliya; 224917 (LWG), 17.iv.2003, roadside, Katerniaghat range, coll. S.D. Maliya; 226606 (LWG), 29.xii.2005, Kuthiyaghat forest, coll. S.D. Maliya. 
Elytraria acaulis (L.f.) Lindau, Nat. Pflanzenfam 1: 304. 1897; Brem., Reinwardtia 3: 250. 1955; Saini, J. Econ. Taxon. Bot. 29 (4): 848. 2005.

Justisia acaulis L. f., Suppl. PI. 84. 1781. Tubiflora acaulis (L. f.) Kuntze, Rev. Gen. PI. 2: 500. 1891; Duthie, FI. Upp. Gang. Plain 2: 51. repr. ed. 1960. Elytraria crenata Vahl, Enum. PI. 1: 106. 1804; Clarke in Hook. f., Fl. Brit. India 4: 394. 1884.

Habit: Herb.

Specimens examined: Not observed. Record based on Saini (2005)

Eranthemum pulchellum Andrews, Bot. Repos. 2: t. 88. 1800; Raizada \& Saxena, Fl. Muss. 1: 551. 1978; Maliya \& Datt, J. Econ. Taxon. Bot. 34 (1): 59. 2010.

Justicia nervosa Vahl, Enum. PI. 1: 164. 1804. Eranthemum nervosum (Vahl) R. Br. ex Roem. \& Schult., Syst. Veg. 1: 174. 1817; Saini, J. Econ. Taxon. Bot. 29 (4): 848. 2005. Daedalacanthus nervosus (Vahl) Anders., J. Linn. Soc. 9: 487. 1867; Clarke in Hook. f., Fl. Brit. India 4: 418. 1884; Duthie, Fl. Upp. Gang. Plain 2: 63. repr. ed. 1960.

Habit: Herb.

Specimens examined: 225910 (LWG), 18.iii.2004, Kuthiyaghat forest, coll. S.D. Maliya.

Eranthemum purpurascens Nees in Wall., PI. Asiat. Rar. 3: 106. 1832; Maliya \& Datt, J. Econ. Taxon. Bot. 34 (1): 59. 2010.

Habit: Herb.

Specimens examined: 225916 (LWG), 19.iii.2004, near Bichhiya Railway Station, coll. S.D. Maliya.

Hemigraphis hirta (Vahl) T. Anders., J. Linn. Soc. Bot. 9: 462. 1867; Clarke in Hook. f., Fl. Brit. India 4: 422. 1884; Duthie, FI. Upp. Gang. Plain 2: 60. repr. ed. 1960; Panigrahi et al., Bull. Bot. Surv. India 11 (1 \& 2): 107. 1969; Maliya \& Datt, J. Econ. Taxon. Bot. 34 (1): 59. 2010.

Ruellia hirta Vahl, Symb. Bot. 3: 84. t. 67. 1796.

Habit: Herb.

Specimens examined: 13430 (LWG), 26.v.1954, Motipur range, coll. Hira Lal; 225945 (LWG), 24.iii.2004, Murtiha range, Katerniaghat Wildlife Sanctuary, Bahraich coll. S.D. Maliya; 227187 (LWG), 27.xii.2005, near Lahaurea Village, Nishangara range, coll. S.D. Maliya; 226609 (LWG), 11.iv.2006, near Lahaurea Village, Nishangara range, coll. S.D. Maliya.

Hemigraphis rupestris Heyne ex T. Anders., J. Linn. Soc. Bot. 9: 462. 1867; Bemk., Vern. Kon. Ned. Ak. Wet. A dam Sect. 2. 41: 1. 141. 1944; Maliya \& Datt, J. Econ.
Taxon. Bot. 34 (1): 59. 2010.

Habit: Herb.

Specimens examined: 224999 (LWG), 22.iv.2003, Nishangara range, coll. S.D. Maliya.

Hygrophila auriculata (Schumach.) Heine, Kew Bull. 16: 172. 1962.

Barleria auriculata Schumach., Beskr. Guin. PI. 285. 1827.

Habit: Annual herb.

Specimens examined: 263688 (LWG), 07.xi.2011, Indo-Nepal border, Nishangara range.

Hygrophila pinnatifida (Dalz.) Sreem., Bull. Bot. Surv. India 10: 222. 1969; Kuntze, Gen. PI. 2: 500. 1891; Heinein, Kew Bull. 16: 177 et Seq. 1962; Saini, J. Econ. Taxon. Bot. 29 (4): 850. 2005.

Nomaphila pinnatifidium Dalz., Kew J. Bot. 3: 38. 1851. Adensoma pinnatifidium (Dalz.) T. Anders., J. Linn. Soc. 9: 445. 1867.

Habit: Herb.

Specimens examined: Not observed. Record based on Saini (2005).

Hygrophila polysperma (Roxb.) Anders., J. Linn. Soc. Bot. 9: 456. 1867; Clarke in Hook. f., Fl. Brit. India 4: 406. 1884; Duthie, Fl. Upp. Gang. Plain 2: 56. repr. ed. 1960.

Justicia polysperma Roxb., Fl. Ind. 1: 119. 1832. Hemiadelphis polyspermus (Roxb.) Nees in Wall., PI. Asiat. Rar. 3: 80. 1832; Panigrahi et al., Bull. Bot. Surv. India 11 (1 \& 2): 107. 1969.

Habit: Herb.

Specimens examined: 2873 (BSA), 12.iii.1964, Murtiha gate, coll. G. Panigrahi; 8023 (BSA), 16.ii.1965, Katrniaghat wildlife sanctuary, Bahraich, O.P. Mishra; 252848 (LWG), 04.iii.2011, Kakrha range, coll. A. Kumar, O. Bajapi \& L.B. Chaudhary.

Hygrophila ringens (L.) R. Br. ex Steud., Nomencl. Bot. 1: 418. 1821.

Ruellia ringens L., Sp. PI. 2: 635. 1753. R. salicifolia Vahl, Symb. Bot. 3: 84. 1794. Hygrophila salicifolia (Vahl) Nees in Wall., PI. Asiat. Rar. 3: 81. 1832; Maliya \& Datt, J. Econ. Taxon. Bot. 34 (1): 59. 2010.

Habit: Herb.

Specimens examined: Not observed. Record based on Maliya \& Datt (2010).

Justicia adhatoda L., Sp. PI. 15. 1753; Duthie, Fl. Upp. Gang. Plain 2: 76. repr. ed. 1960.

Adhatoda vasica Nees in Wall., PI. Asiat. Rar. 3: 
102. 1832; Clarke in Hook. f. Fl. Brit. India 4: 540. 1885; Panigrahi et al., Bull. Bot. Surv. India 11(1 \& 2): 107. 1969. Habit: Shrub.

Specimens examined: 7924 (BSA), 10.ii.1965 Nishangara range, coll. O.P. Mishra.

Justicia gendarussa Burm. f., Fl. Ind. 10. 1768; Duthie, Fl. Upp. Gang. Plain 2: 78. repr. ed. 1960; Maliya \& Datt, J. Econ. Taxon. Bot. 34(1): 59. 2010.

Gendarussa vulgaris Nees in Wall., PI. Asiat. Rar. 3: 104. 1832; Bremk., Densk Bot. Arkiv. 20: 81. 1961.

Habit: Shrub.

Specimens examined: 225933 (LWG), 20.iii.2004, Girijapuri forest, coll. S.D. Maliya.

Justicia micrantha (Oerst.) Graham, Kew Bull. 43: 603 1988; Panigrahi et al., Bull. Bot. Surv. India 11 (1 \& 2): 107. 1969.

Chaetothylopsis micrantha Oerst. Vidensk. Meddel. Dansk Naturhist. Foren. Kjøbenhavn 1854 (8-12): 163164. 1854.

Habit: Herb.

Specimens examined: 09.ii.1965, Bhagahan nala, Motipur range, O.P. Mishra 7768 (BSA).

Justicia procumbens L., Sp. PI. 15. 1753; Maliya \& Datt, J. Econ. Taxon. Bot. 34 (1): 60. 2010.

Habit: Herb.

Specimens examined: 26.x.2004, Murtiha range, coll. S.D. Maliya 227125 (LWG).

Justicia quinqueangularis Koenig ex Roxb., Fl. Ind. 1: 134. 1820; Duthie, Fl. Upp. Gang. Plain 2: 77. repr. ed. 1960; Panigrahi et al., Bull. Bot. Surv. India 11 (1 \& 2): 107. 1969.

Habit: Herb.

Specimens examined: 22.xi.1964, Motipur range, G. Panigrahi \& O.P. Mishra 6539 (BSA).

Lepidagathis incurva Buch.-Ham. ex D. Don, Prodr. Fl. Nepal. 119. 1825; Maliya, J. Econ. Taxon. Bot. 36 (2): 423. 2012.

Habit: Perennial herb.

Specimens examined: 15.iii.2010, Rampurwa, Katerniaghat Wildlife Sanctuary, Bahraich coll. S.D. Maliya 252615 (LWG); 21.iv.2010, road side, Bichhiya to Katerniaghat road, Katerniaghat Wildlife Sanctuary, L.B. Chaudhary, A. Kumar \& O. Bajpai 252233 (LWG).

Nelsonia canescens (Lam.) Spreng., Syst. Veg. 1: 42 1824
Justicia canescens Lam., Encycl. 1: 41. 1791. Nelsonia campestris R. Br., Prodr. Fl. Nov. Holl. 481. 1810; Duthie, Fl. Upp. Gang. Plain 2: 52. repr. ed. 1960; Panigrahi et al., Bull. Bot. Surv. India 11 (1 \& 2): 107. 1969.

Habit: Herb or under shrub.

Specimens examined: 14.iii.1964, Rampurwa, G. Panigrahi 2894 (BSA).

Peristrophe paniculata (Forssk.) Brumitt, Kew Bull. 38: 451. 1983; Maliya \& Datt, J. Econ. Taxon. Bot. 34 (1): 60. 2010

Dianthera paniculata Forssk., Fl. Aegypt.-Arab. 7. 1775. D. bicalyculata Retz., Acta Holm. 1775: 279. 1776. Peristrophe bicalyculata (Retz.) Nees. in Wall., Pl. Asiat. Rar. 3: 113. 1832; Clarke in Hook. f., Fl. Brit. India 4: 554. 1885; Duthie, Fl. Upp. Gang. Plain 2: 79. repr. ed. 1960; Panigrahi et al., Bull. Bot. Surv. India 11 (1 \& 2): 107. 1969; Saini, J. Econ. Taxon. Bot. 29 (4): 851. 2005.

Habit: Herb or undershrub.

Specimens examined: 28.xii.2003, Katerniaghat range, coll. S.D. Maliya 225280 (LWG).

Ruellia prostrata Poir., Encycl. 6: 349. 1804.

Dipteracanthus prostratus (Poir.) Nees in Wall., PI. Asiat. Rar. 3: 81. 1832; Panigrahi et al., Bull. Bot. Surv. India 11 (1 \& 2): 107. 1969; Saini, J. Econ. Taxon. Bot. 29 (4): 848. 2005

Habit: Undershrub.

Specimens examined: Not observed. Record based on Panigrahi et al. (1969) and Saini (2005).

Rungia pectinata (L.) Nees in DC, Prodr. 11: 470. 1847; Panigrahi et al., Bull. Bot. Surv. India 11 (1 \& 2): 107. 1969.

Justicia pectinata L., Amoen. Acad. 4: 299. 1760. Rungia parviflora var. pectinata (L.) Clarke in Hook. f., Fl. Brit. India 4: 550. 1885; Duthie, FI. Upp. Gang. Plain 2: 80. repr. ed. 1960

Habit: Annual herb.

Specimens examined: Not observed. Record based on Panigrahi et al. (1969).

Rungia repens (L.) Nees in Wall., PI. Asiat. Rar. 3: 110. 1832; Duthie, Fl. Upp. Gang. Plain 2: 80. repr. ed. 1960; Panigrahi et al., Bull. Bot. Surv. India 11 (1 \& 2): 107. 1969; Saini, J. Econ. Taxon. Bot. 29 (4): 851. 2005.

Justicia repens L., Sp. PI. 15. 1753.

Habit: Herb.

Specimens examined: Not observed. Record based on Panigrahi et al. (1969) and Saini (2005). 
Staurogyne glauca Kuntze, Revis. Gen. PI. 2: 497. 1891.

Ebermaiera glauca Nees, Companion Bot. Mag. 2: 310. 1837; Panigrahi et al., Bull. Bot. Surv. India 11 (1 \& 2): 107. 1969.

Habit: Herb.

Specimens examined: Not observed. Record based on Panigrahi et al. (1969).

Staurogyne glutinosa Kuntze, Revis. Gen. PI. 2: 497 1891; Duthie, Fl. Upp. Gang. Plain 2: 53. repr. ed. 1960.

Ebermaiera glutinosa Wall. ex Clarke in Hook. f., Fl. Brit. India 4: 396. 1884; Panigrahi et al., Bull. Bot. Surv. India 11 (1 \& 2): 107. 1969.

Habit: Herb.

Specimens examined: 14.ii.1965, Uraital, O.P. Mishra 8000 (BSA).

\section{AMARANTHACEAE}

Achyranthes aspera L., Sp. PI. 204. 1753; Saini, J. Econ. Taxon. Bot. 29 (4): 860. 2005; Maliya \& Datt, J. Econ. Taxon. Bot. 34 (1): 61.2010.

Habit: Herb.

Specimens examined: 21.v.1987, K.K. Singh \& party 5985 (LWG); 9.xi.2002, near Bichhiya railway station, Katerniaghat range, coll. S.D. Maliya 224516 (LWG); 10.xi.2002, Murtiha range, coll. S.D. Maliya 224584 (LWG).

Achyranthes aspera L. var. prophyristachya (Wall. ex Moq.) Hook. f., Fl. Brit. India 4: 730. 1885; Raizada, Suppl. FI. Upp. Gang. Plain 228. 1976; Saini, J. Econ. Taxon. Bot. 29 (4): 860. 2005; Maliya \& Datt, J. Econ. Taxon. Bot. 35 (1): 61.2010.

Achyranthes porphyristachya Wall. ex Moq., Prodr. 13(2): 316. 1849.

Habit: Herb.

Specimens examined: 07.iv.2009, Kakraha range, L.B. Chaudhary, A. Kumar \& O. Bajapi 250763 (LWG); 24.x.2009, Kakraha range, L.B. Chaudhary, A. Kumar \& O. Bajpai 250686 (LWG).

Aerva javanica (Burm. f.) Juss. (Ann. Mus. Natl. Hist Nat. 2: 131. 1803) ex Schult., Ann. Mus. Hist. Nat. 11: 131 1808; Hook. f. in Hook. f., Fl. Brit. India 4: 727. 1885; Saini, J. Econ. Taxon. Bot. 29 (4): 861. 2005.

Iresine javanica Burm. f., Fl. Ind. 212, t. 65, f. 2. 1768.

Habit: Herb or undershrub.

Specimens examined: Not observed. Record based on Saini (2005)
Aerva lanata (L.) Juss. ex Schult., Syst. Veg. 5: 564. 1819; Hook. f. in Hook. f., Fl. Brit. India 4: 728. 1885; Duthie, Fl. Upp. Gang. Plain 3: 139. repr. ed. 1960; Maliya \& Datt, J. Econ. Taxon. Bot. 34 (1): 61. 2010.

Achyranthes lanata L., Sp. PI. 204. 1753.

Note: A. lanata (L.) Juss., Ann. Mus. Natl. Hist. Nat. 2: 131. 1803, comb. invl.

Habit: Herb or undershrub.

Specimens examined: 12.xi.2002, Nishangara range, coll. S.D. Maliya 224639 (LWG).

Aerva sanguinolenta (L.) BI., Bijdr. 547. 1826; Panigrahi et al., Bull. Bot. Surv. India 11 (1 \& 2): 103. 1969; Maliya \& Datt, J. Econ. Taxon. Bot. 34 (1): 61. 2010.

Achyranthes sanguinolenta L., Sp. PI. 294. 1762.

Habit: Herb.

Specimens examined: 08.ii.1965, Motipur range, O.P. Mishra 7732 (BSA); 23.viii.2002, Nishangara range, coll. S.D. Maliya 223606 (LWG); 10.xi.2002, Murtiha range, coll. S.D. Maliya 224577 (LWG); 11.xi.2002, Murtiha range, coll. S.D. Maliya 224607 (LWG); 16.iii.2008, near Bichhiya railway station, Katerniaghat range, coll. S.D. Maliya 218513 (LWG).

Alternanthera sessilis (L.) DC., Cat. PI. Horti Monsp. 77. 1813; Panigrahi et al., Bull. Bot. Surv. India 11 (1 \& 2): 104. 1969; Maliya \& Datt, J. Econ. Taxon. Bot. 34 (1): 61. 2010.

Gomphrena sessilis L., Sp. PI. 225. 1753.

Habit: Prostrate herb.

Specimens examined: 29.viii.2002, Murtiha range, coll. S.D. Maliya 223799 (LWG); 31.viii.2003, Fakirpur village, Nishangara range, coll. S.D. Maliya 214878 (LWG).

*Amaranthus caudatus L., Sp. PI. 990. 1753; Duthie, Fl. Upp. Gang. Plain 3: 134. repr. ed. 1960; Panigrahi et al., Bull. Bot. Surv. India 11 (1 \& 2): 104. 1969.

Habit: Herb, cultivated.

Specimens examined: 25.xi.1964, Plantation area, Katerniaghat range, G. Panigrahi \& O.P. Mishra 6612 (BSA).

**Amaranthus hybridus L., Sp. PI. 990. 1753.

A. hybridus L. var. paniculatus (L.) Uline \& Bray, Mem. Torrey Bot. Club 5 (11): 145. 1894; Panigrahi et al., Bull. Bot. Surv. India 11 (1 \& 2): 104. 1969.

A. paniculatus L., Sp. PI. ed. 2. 2: 1406. 1763.

Habit: Herb.

Specimens examined: 25.xi.1964, Katernighat plantation area, G. Panigrahi \& O.P. Mishra 6613 (LWG). 
Amaranthus spinosus L., Sp. PI. 991. 1753; Hook. f. in Hook. f., Fl. Brit. India 4: 718. 1885; Duthie, Fl. Upp. Gang. Plain 3: 133. repr. ed. 1960; Panigrahi et al., Bull. Bot. Surv. India 11 (1 \& 2): 104. 1969; Maliya \& Datt, J. Econ. Taxon. Bot. 34 (1): 61. 2010.

Habit: Herb.

Specimens examined: 24.viii.2002, Nishangara range, coll. S.D. Maliya 223672 (LWG); 09.xi.2002, near Bichhiya town, Katerniaghat range, Bahraich, coll. S.D. Maliya 224507 (LWG); 24.xii.2003, Kuthiyaghat forest, coll. S.D. Maliya 225257 (LWG).

**Amaranthus tricolor L., Sp. PI. 989. 1753.

A. gangeticus L., Syst. Nat. ed. 10. 2: 1268. 1759.

Habit: Herb.

Specimens examined: 26.v.1954, Motipur range, Hira Lal 13437 (LWG).

Amaranthus viridis L., Sp. Pl. 1405. 1763; Duthie, FI. Upp. Gang. Plain 3: 136. repr. ed. 1960; Maliya \& Datt, J. Econ. Taxon. Bot. 34 (1): 61.2010.

Habit: Herb.

Specimens examined: 29.viii.2002, Murtiha range, coll. S.D. Maliya 223798 (LWG).

**Celosia argentea L., Sp. Pl. 205. 1753; Hook. f. in Hook. f., Fl. Brit. India 4: 714. 1885; Duthie, Fl. Upp. Gang. Plain 3: 131. repr. ed. 1960; Panigrahi et al., Bull. Bot. Surv. India 11 (1 \& 2): 104. 1969; Maliya \& Datt, J. Econ. Taxon. Bot. 34 (1): 61. 2010.

Habit: Annual herb.

Specimens examined: 28.x.2004, Lahaura village, Nishangara range, coll. S.D. Maliya 227132 (LWG).

*Celosia argentea L. var. cristata (L.) Kuntze, Revis. Gen. PI. 2: 541. 1891; Panigrahi et al., Bull. Bot. Surv. India 11 (1 \& 2): 104. 1969.

C. cristata L., Sp. PI. 205. 1753; Duthie, Fl. Upp. Gang. Plain 3: 131. repr. ed. 1960.

Habit: Herb, cultivated.

Specimens examined: 26.x.2004, Murtiha range, coll. S.D. Maliya 227115 (LWG); 27.xii.2005, Lahaura village, Nishangara range, coll. S.D. Maliya 227194 (LWG).

**Deeringia amaranthoides (Lam.) Merr., Interpr. Herb. Amboin. 211. 1917; Panigrahi et al., Bull. Bot. Surv. India 11 (1 \& 2): 104. 1969.

Achyranthes amaranthoides Lam., Encycl. 1: 548. 1785.

Habit: Scandent shrub.

Specimens examined: 23.xii.2003, Girijapuri forest, coll. S.D. Maliya 225238 (LWG).

Digera muricata (L.) Mart., Nova Acta Phys.-Med. Acad. Caes. Leop.-Carol. Nat. Cur. 13: 285. 1826; Maliya \& Datt, J. Econ. Taxon. Bot. 34 (1): 61. 2010.

Achyranthes muricata L., Sp. PI. 295. 1762. Digera arvensis Forssk., FI. Aegypt.- Arab. 65. 1775; Hook. f. in Hook. f., Fl. Brit. India 4: 717. 1885; Duthie, Fl. Upp. Gang. Plain 3: 132. repr. ed. 1960.

Habit: Herb.

Specimens examined: 08.ix.2005, Kuthiyaghat forest, coll. S.D. Maliya 227162 (LWG).

\section{ANACARDIACEAE}

Buchanania cochinchinensis (Lour.) Almeida, Fl. Maharashtra 1: 287. 1996.

Toluifera cochinchinensis Lour., Fl. Cochinch. 1: 262. 1790. Buchanania lanzan Spreng., J. Bot. (Schrader) 1800 (2): 234. 1801; Panigrahi et al., Bull. Bot. Surv. India 11 (1 \& 2): 101. 1969; Saini, J. Econ. Taxon. Bot. 29 (3): 556. 2005. B. latifolia Roxb., Fl. Ind. 2: 385. 1832; Hook. f. in Hook. f., Fl. Brit. India 2: 23. 1879.

Habit: Tree.

Specimens examined: 12.ii.1965, Rampurwa, Nishangara range, O.P. Mishra 7942 (BSA).

Lannea coromandelica (Houtt.) Merr., J. Arn. Arb. 19: 353. 1938; Panigrahi et al., Bull. Bot. Surv. India 11 (1 \& 2): 101. 1969; Maliya \& Datt, J. Econ. Taxon. Bot. 34 (1): 50. 2010.

Dialium coromandelicum Hautt., Nat. Hist. 2(2): 39, t. 5, f. 2. 1774. Odina wodier Roxb., Fl. Ind. 2: 293. 1832; Hook. f. in Hook. f., Fl. Brit. India 2: 29. 1879.

Habit: Tree.

Specimens examined: 15.iii.1964, Rampurwa, Nishangara range, G. Panigrahi 2910 (BSA); 17.iv.2003, road side, Katerniaghat range, coll. S.D. Maliya 224904 (LWG); 20.iv.2003, Nepal border, Murtiha range, coll. S.D. Maliya 224944 (LWG); 22.iv.2003, Nishangara range, coll. S.D. Maliya 224984 (LWG) (Image 1).

*Mangifera indica L., Sp. Pl. 200. 1753; Hook. f. in Hook. f., Fl. Brit. India 2: 13. 1879; Duthie, Fl. Upp. Gang. Plain 1: 176. repr. ed. 1960; Maliya \& Datt, J. Econ. Taxon. Bot. 34 (1): 50. 2010.

Habit: Tree, cultivated

Specimens examined: 17.iv.2003, Katerniaghat range, coll. S.D. Maliya 224905 (LWG) 24.iv.2003, Murtiha range, coll. S.D. Maliya 214826 (LWG). 
Semecarpus anacardium L. f., Suppl. PI. 182. 1781; Hook. f. in Hook. f., Fl. Brit. India 2: 30. 1879; Saini, J. Econ. Taxon. Bot. 29 (3): 557. 2005; Maliya \& Datt, J. Econ. Taxon. Bot. 34 (1): 50. 2010.

Habit: Tree.

Specimens examined: 08.xii.1986, Bichhia, K.K. Khanna \& R. Saran 37635 (BSA); 25.viii.2002, Murtiha range, coll. S.D. Maliya 223718 (LWG).

*Spondias pinnata (J. Koenig ex L. f.) Kurz, Prelim. Rep. Forest Pegu, App. A: 44. 1875.

Mangifera pinnata J. Koenig ex L. f., Suppl. PI. 156. 1782.

Habit: Tree, cultivated.

Specimens examined: 04.iv.2011, rest house, Katerniaghat range, A. Kumar \& O. Bajpai 263620 (LWG).

\section{ANNONACEAE}

*Annona squamosa L., Sp. PI. 537. 1753; Hook. f. \& Thoms. in Hook. f., Fl. Brit. India 1: 78. 1872; Mitra in Sharma et al., Fl. India 1: 207. 1993; Maliya \& Datt, J. Econ. Taxon. Bot. 34 (1): 46. 2010.

Habit: Shrub or small tree, cultivated.

Specimens examined: 24.iv.2003, chhawni forest, Murtiha range, coll. S.D. Maliya 214837 (LWG); 27.x.2004, Katerniaghat range, coll. S.D. Maliya 227130 (LWG).

*Artabotrys hexapetalus (L. f.) Bhandari, Baileya 12: 147. 1964; Mitra in Sharma et al., FI. India 1: 251. 1993; Saini, J. Econ. Taxon. Bot. 29 (3): 534. 2005.

Annona hexapetala L. f., Suppl. PI. 270. 1781.

Habit: Scandent shrub, cultivated.

Specimens examined: Not observed. Record based on Saini (2005)

Miliusa tomentosa (Roxb.) Sinclair, Gard. Bull. Singapore 14: 378. 1955; Mitra in Sharma et al., Fl. India 1: 221. 1993; Saini, J. Econ. Taxon. Bot. 29 (3): 534. 2005.

Uvaria tomentosa Roxb., Pl. Coromandel 1: $31, \mathrm{t}$ 35. 1795. Saccopetalum tomentosum (Roxb.) Hook. f. \& Thoms., Fl. India 152. 1855 \& in Hook. f ., Fl. Brit. India 1: 88. 1872.

Habit: Tree.

Specimens examined: 27.iv.2010, near Balcha barrier, Kakraha range, A. Kumar \& O. Bajpai 252280 (LWG).

Miliusa velutina (Dunal.) Hook. f. \& Thoms., Fl. Ind. 1: 151. 1855 \& in Hook. f., Fl. Brit. India 1: 87. 1872; Mitra in Sharma et al., Fl. India 1: 251. 1993; Saini, J. Econ. Taxon. Bot. 29 (3): 535. 2005.

Uvaria velutina Dunal., Anon. 91. 1817. U. villosa

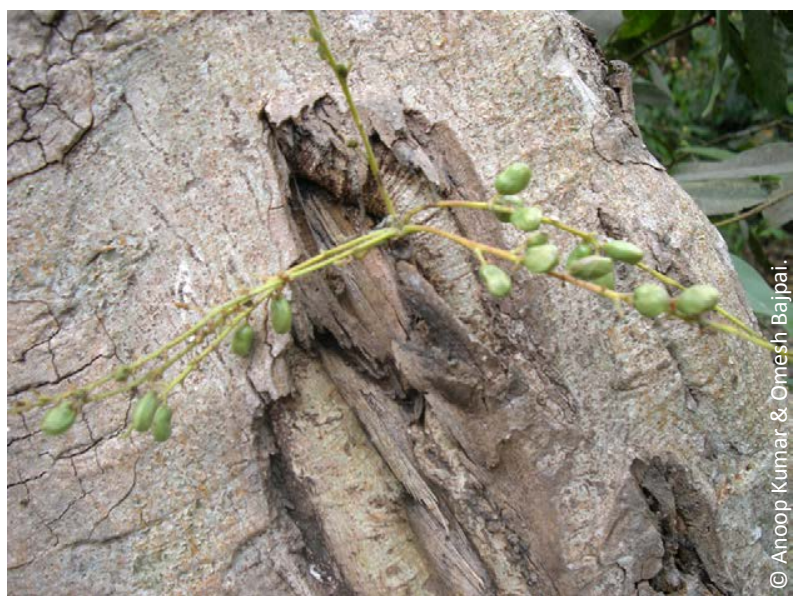

Image 1. Lannea coromandelica (Houtt.) Merr.

Roxb., Fl. Ind. 2: 668. 1825. Guatteria velutina (Dunal.) A. DC., Mem. Soc. Genev. 5: 42. 1932.

Habit: Tree.

Specimens examined: 08.vi.2009, Tiger chowk, Nishangara range, L.B. Chaudhary, A. Kumar \& O. Bajpai 250620 (LWG); 08.vi.2009, near Balcha barrier, Kakraha range, L.B. Chaudhary, A. Kumar \& O. Bajpai 252825 (LWG).

*Polyalthia longifolia (Sonn.) Thw., Enum. Pl. Zeyl. 398. 1864; Hook. f. \& Thoms. in Hook. f., Fl. Brit. India 1: 62. 1872; Mitra in Sharma et al., Fl. India 1: 274. 1993; Saini, J. Econ. Taxon. Bot. 29 (3): 535. 2005; Maliya \& Datt, J. Econ. Taxon. Bot. 34 (1): 46. 2010.

Uvaria longifolia Sonn., Vey. Indes. 2: 233. t. 131. 1782.

Habit: Tree, cultivated.

Specimens examined: 22.iv.2010, Bichhiya railway station, A. Kumar \& O. Bajapi 252269 (LWG).

Polyalthia suberosa (Roxb.) Thwaites, Enum. Pl. Zeyl. 398. 1864; Hook. f. \& Thoms. in Hook. f., Fl. Brit. India 1: 65. 1872; Panigrahi et al., Bull. Bot. Surv. India 11 (1 \& 2): 93. 1969; Mitra in Sharma et al., FI. India 1: 278. 1993.

Uvaria suberosa Roxb., PI. Coromandel 1: t. 34. 1795.

Habit: Shrub or small tree.

Specimens examined: 25.v.1954, Motipur range, Hira Lal 13405 (LWG); 21.xi.1964, Nishangara range, G. Panigrahi \& O.P. Mishra 6520 (BSA); 15.iv.1967, S. L. Kapoor 70045 (LWG); 24.viii.2002, Nishangara range, K.K. Singh \& coll. S.D. Maliya 223682 (LWG); 09.xi.2002, road side, Katerniaghat range, K.K. Singh \& coll. S.D. Maliya 224542 (LWG); 27.xii.2005, Girijapuri forest, coll. S.D. Maliya 227200 (LWG); 24.iv.2010, near Lakkadshah baba majar, A. Kumar, O. Bajpai \& L.B. Chaudhary 252210 


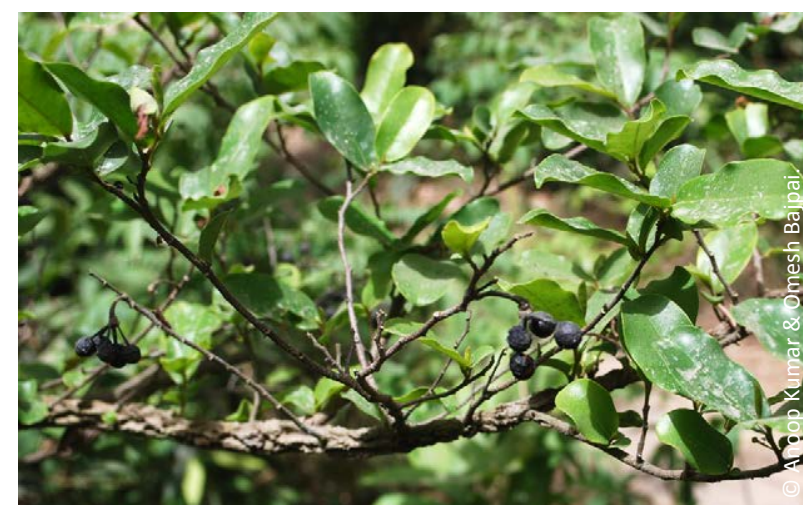

Image 2. Polyalthia suberosa (Roxb.) Thwaites

(LWG) (Image 2).

Uvaria hamiltoni Hook. f. \& Thoms., Fl. Ind. 1: 96. 1855 \& in Hook. f., Fl. Brit. India 1: 48. 1872; Mitra in Sharma et al., Fl. India 1: 290. 1993; Saini, J. Econ. Taxon. Bot. 29 (3): 535. 2005.

Habit: Evergreen scandent shrub.

Specimens examined: Not observed. Record based on Saini (2005).

\section{APIACEAE}

Centella asiatica (L.) Urban in Mart., Fl. Brass. 11(1): 287, t. 78. 1879; Panigrahi et al., Bull. Bot. Surv. India 11 (1 \& 2): 105. 1969; Saini, J. Econ. Taxon. Bot. 29 (3): 588. 2005; Maliya \& Datt, J. Econ. Taxon. Bot. 34 (1): 54.2010.

Hydrocotyle asiatica L., Sp. PI. 234. 1753; Clarke in Hook. f., Fl. Brit. India 2: 669. 1879; Duthie, Fl. Upp. Gang. Plain 1: 358. repr. ed. 1960.

Habit: Prostrate herb.

Specimens examined: 20.xi.1964, Nishangara range, G. Panigrahi \& O.P. Mishra 6523 (BSA); 23.v.1990, Vardia, K.K. Khanna 89 (BSA); 23.viii.2002, Nishangara range, coll. S.D. Maliya 223627 (LWG); 17.iv.2003, rest house, Katerniaghat range, coll. S.D. Maliya 224915 (LWG); 20.iv.2003, Murtiha range, coll. S.D. Maliya 224949 (LWG); 22.iv.2003, Nishangara range, coll. S.D. Maliya 224987 (LWG).

*Coriandrum sativum L., Sp. PI. 1: 256. 1753; Maliya, J. Econ. Taxon. Bot. 36 (2): 421. 2012.

Habit: Annual herb, cultivated, found as an escape.

Specimens examined: Not observed. Record based on Maliya \& Datt (2012).

*Cuminum cyminum L., Sp. PI. 254. 1753; Clarke in Hook. f., Fl. Brit. India 2: 718. 1879; Saini, J. Econ. Taxon. Bot. 29(3): 588. 2005.
C. odorum Salisb., Prodr. Stirp. Chap. Allerton 165. 1796.

Habit: Herb, cultivated, found as an escape.

Specimens examined: Not observed. Record based on Saini (2005).

*Foeniculum vulgare Mill., Gard. dict. ed. 8: Foeniculum no. 1. 1768; Clarke in Hook. f., Fl. Brit. India 2: 695. 1879; Saini, J. Econ. Taxon. Bot. 29(3): 588. 2005.

Habit: Herb, cultivated, found as an escape.

Specimens examined: Not observed. Record based on Saini (2005).

**Oenanthe javanica (BI.) DC., Prodr. 4: 138. 1830; Saini, J. Econ. Taxon. Bot. 29 (3): 589. 2005; Maliya \& Datt, J. Econ. Taxon. Bot. 34 (1): 54. 2010.

Sium javanicum Bl., Bijdr. Fl. Ned. Ind. 15: 881. 1826. Oenanthe stolonifera Wall. ex DC., Prodr. 4: 138. 1830.

Habit: Herb.

Specimens examined: Not observed. Record based on Saini (2005) and Maliya \& Datt (2010).

\section{APOCYNACEAE}

*Allamanda cathartica L., Mant. PI. 2: 214. 1771; Saini, J. Econ. Taxon. Bot. 29 (3): 609. 2005.

Habit: Scandent shrub, cultivated.

Specimens examined: Not observed. Record based on Saini (2005).

*Alstonia scholaris (L.) R. Br., Asclepiadeae 64. 1810; Hook. f. in Hook. f., Fl. Brit. India 3: 642. 1882; Duthie, Fl. Upp. Gang. Plain 1: 486. repr. ed. 1960; Saini, J. Econ. Taxon. Bot. 29 (3): 609. 2005; Maliya \& Datt, J. Econ. Taxon. Bot. 34 (1): 56. 2010.

Echites scholaris L., Mant. PI. 1: 53. 1767.

Habit: Small or medium sized evergreen tree, cultivated.

Specimens examined: 20.iii.2004, Girijapuri forest, coll. S.D. Maliya 225918 (LWG); 16.vii.2004, Kuthiyaghat forest, coll. S.D. Maliya 225953 (LWG); 19.vii.2004, Kuthiyaghat forest, coll. S.D. Maliya 225973 (LWG); 20.iv.2004, Girijapuri forest, coll. S.D. Maliya 227107 (LWG).

Calotropis gigantea (L.) R. Br. in W. T. Aiton, Hort. Kew. (ed. 2) 2: 78. 1811; Hook. f. in Hook. f., Fl. Brit. India 4: 17. 1883; Duthie, Fl. Upp. Gang. Plain 1: 497. repr. ed. 1960.

Asclepias gigantea L., Sp. PI. 214. 1753.

Habit: Shrub or small tree.

Specimens examined: 26.xi.1987, K.K. Singh \& party 


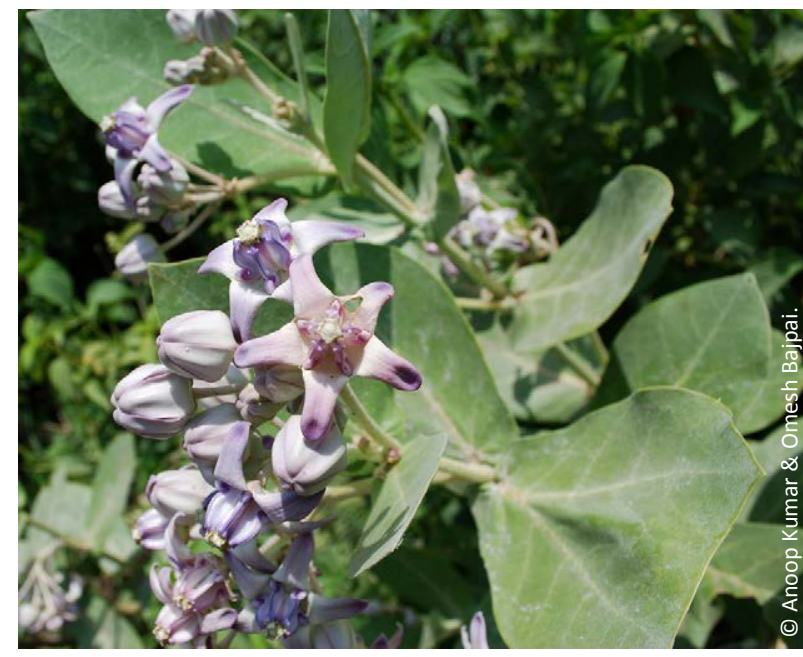

Image 3. Calotropis gigantea (L.) R. Br.

6901 (LWG); 17.iii.2004, Kuthiyaghat forest, coll. S.D. Maliya 225904 (LWG) (Image 3).

Calotropis procera (Ait.) Ait., Hortus Kew. (ed. 2) 2: 78. 1811; Duthie, Fl. Upp. Gang. Plain 1: 498. repr. ed. 1960; Saini, J. Econ. Taxon. Bot. 29 (3): 613. 2005; Maliya \& Datt, J. Econ. Taxon. Bot. 34 (1): 56. 2010.

Asclepias procera Ait., Hort. Kew. 1: 305. 1789.

Habit: Shrub.

Specimens examined: 22.v.1986, Hasulia, K.K. Khanna \& R. Saran 37111 (BSA); 09.xi.2002, near Bichhiya, coll. S.D. Maliya 224520 (LWG); 17.iv.2003, Katerniaghat range, coll. S.D. Maliya 224909 (LWG); 22.iv.2003, Nishangara range, coll. S.D. Maliya 224973 (LWG); 16.iii.2008, Murtiha range, coll. S.D. Maliya 218516 (LWG) (Image 4).

Carissa spinarum L., Mant. PI. 2: 559. 1771; Duthie, FI. Upp. Gang. Plain 1: 483. repr. ed. 1960.

C. congesta Wight, Icon. PI. Ind. Orient. 4: t. 1289. 1848; Maliya \& Datt, J. Econ. Taxon. Bot. 34 (1): 56. 2010. Habit: Shrub.

Specimens examined: 28.i.2005, Murtiha range, coll. S.D. Maliya 224690 (LWG).

Cryptolepis buchanani Schult. in Roem. \& Schult., Syst. Veg. 4: 409. 1819; Maliya \& Datt, J. Econ. Taxon. Bot. 34 (1): 57. 2010.

Habit: Large twining shrub.

Specimens examined: 26.x.2004, Murtiha range, coll. S.D. Maliya 227126 (LWG).

Dregea volubilis (L. f.) Benth. ex Hook. f. in Hook. f., FI. Brit. India 4: 46. 1883; Maliya \& Datt, J. Econ. Taxon. Bot.

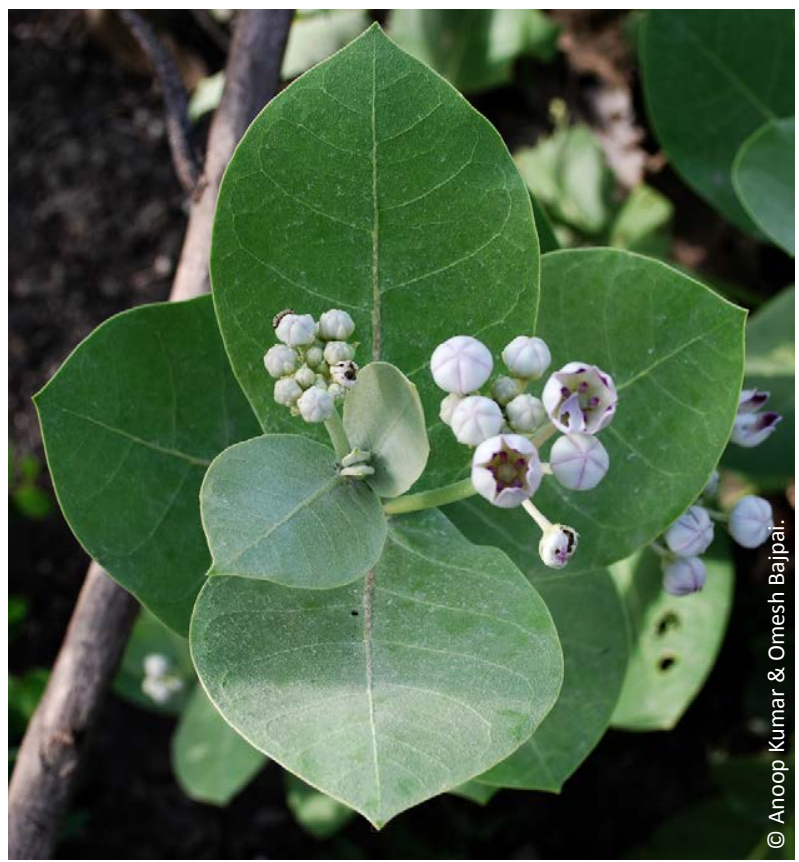

Image 4. Calotropis procera (Ait.) Ait.

34 (1): 57. 2010.

Asclepias volubilis L. f., Suppl. PI. 170. 1782. Marsdenia volubilis (L. f.) Cooke, Fl. Bombay 2:166. 1904. Habit: Twining shrub.

Specimens examined: 13.xi.2002, Nishangara range, coll. S.D. Maliya 224666 (LWG).

Holarrhena pubescens Wall. ex G. Don, Gen. Hist. 4: 78. 1837; Maliya \& Datt, J. Econ. Taxon. Bot. 34 (1): 56. 2010.

Echites antidysentericus Roth in Roem. \& Schult., Syst. Veg. 4: 394. 1819. E. pubescens Buch.-Ham., Trans. Linn. Soc. London 13: 524. 1822, nom. illeg. Holarrhena antidysenterica (Roth) Wall. ex A. DC. in A. P. de Candolle, Prodr. 8: 413. 1844; Saini, J. Econ. Taxon. Bot. 29 (3): 611. 2005.

Habit: Tree.

Specimens examined: 25.v.1954, Motipur forest, Hira Lal 13402 (LWG); 13.ii.1965, Motipur range, K.K. Khanna 39892 (BSA); 27.viii.2002, Murtiha range, coll. S.D. Maliya 223787 (LWG); 08.x.2009, road side, Murtiha range, coll. S.D. Maliya 226659 (LWG) (Image 5).

Ichnocarpus frutescens (L.) Ait., Hort. Kew. 2: 69. 1811; Maliya \& Datt, J. Econ. Taxon. Bot. 34 (1): 56. 2010. Apocynum frutescens L., Sp. PI. 312. 1753.

Habit: Evergreen climbing shrub.

Specimens examined: 65.v.1954, Motipur forest, Hira Lal 13457 (LWG); 08.xii.1986, Nishangara range, K.K. 
Khanna \& R. Saran 37610 (BSA); 16.v.1987, K.K. Singh \& party 5964 (LWG); 24.xi.1987, Rampurwa forest, K.K. Singh \& party 6187 (LWG); 09.xi.2002, near Bichhiya, coll. S.D. Maliya 221519 (LWG); 10.xi.2002, Murtiha range, coll. S.D. Maliya 224589 (LWG); 12.xi.2002, Nishangara range, coll. S.D. Maliya 224657 (LWG); 18.iii.2004, Kuthiyaghat forest, coll. S.D. Maliya 225911 (LWG); 05.xi.2008, Katerniaghat range, coll. S.D. Maliya 226636 (LWG).

**Marsdenia lucida Edgew. ex Madden, J. Asiat. Soc. Bengal, Pt. 2, Nat. Hist. 17(1): 370. 1848; Maliya, J. Econ. Taxon. Bot. 36 (2): 422.2012.

Habit: Evergreen climber.

Specimens examined: 24.viii.2002, Nishangara range, coll. S.D. Maliya 223687 (LWG).

Oxystelma esculentum (L. f.) Sm., Cycl. 25: (unnumbered). 1813.

Periploca esculenta L. f., Suppl. Pl. 168. 1781. Oxystelma esculentum (L. f.) R. Br. ex Schult., Mem. Wern. Soc. 1: 40. 1810; Hook. f. in Hook. f., Fl. Brit. India 4: 17. 1883; Duthie, Fl. Upp. Gang. Plain 1: 500. repr. ed. 1960; Maliya \& Datt, J. Econ. Taxon. Bot. 34 (1): 57. 2010.

Habit: Climbing perennial herb.

Specimens examined: 02.ix.2003, Kuthiyaghat forest, coll. S.D. Maliya 225206 (LWG).

Rauvolfia serpentina (L.) Benth. ex Kurz, Forest FI. Burma 2: 171. 1877; Hook. f. in Hook. f., Fl. Brit. India 3: 632. 1882; Duthie, Fl. Upp. Gang. Plain 1: 484. repr. ed. 1960; Maliya \& Datt, J. Econ. Taxon. Bot. 34 (1): 56. 2010.

Ophioxylon serpentinum L., Sp. PI. 1043. 1753.

Habit: Small shrub.

Specimens examined: 24.xi.1987, K.K. Singh \& party

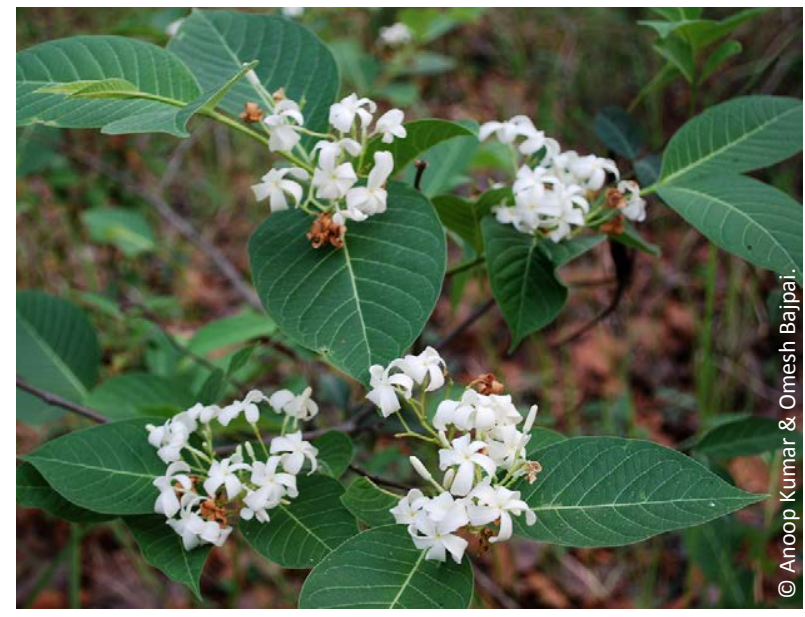

Image 5. Holarrhena pubescens Wall. ex G. Don.
6919 (LWG); 21.v.1990, Bichhiya, K.K. Khanna 39912 (BSA); 26.viii.2002, Katerniaghat range, coll. S.D. Maliya 223765 (LWG); 24.iv.2003, near chawani, Murtiha range, coll. S.D. Maliya 214835 (LWG); 19.vii.2004, Kutihyaghat forest, coll. S.D. Maliya 225967 (LWG); 09.x.2009, Nishangara range, coll. S.D. Maliya 226663 (LWG); 23.x.2010, Girijapuri forest, coll. S.D. Maliya 252628 (LWG).

*Tabernaemontana divaricata (L.) R. Br., Roem. \& Schult., Syst. Veg. 4: 427. 1819; Maliya \& Datt, J. Econ. Taxon. Bot. 34 (1): 56. 2010.

Nerium divaricatum L., Sp. PI. 209. 1753. N. coronarium Jacq., Collectanea 1: 138.1787. Tabernaemontana coronaria (Jacq.) Willd., Enum. PI. 275. 1809; Hook. f. in Hook. f., Fl. Brit. India 3: 646. 1882. Ervatamia coronaria (Jacq.) Stapf in Thiselton-Dyer, Fl. Trop. Afr. 4(1): 127. 1902, Duthie, Fl. Upp. Gang. Plain 1: 488. repr. ed. 1960. E. divaricata (L.) Burkill, Rec. Bot. Surv. India 10: 320. 1925.

Habit: Evergreen shrub, cultivated for ornamental purpose.

Specimens examined: 24.iv.2003, rest house, Murtiha range, coll. S.D. Maliya 214830 (LWG); 16.iii.2004, Karmohini forest, coll. S.D. Maliya 225295 (LWG).

**Thevetia peruviana (Pers.) Schum. in Engler \& Prantl, Nat. Pflanzenfam. 4(2): 159. 1895; Saini, J. Econ. Taxon. Bot. 29 (3): 612.2005

Cerbera peruviana Pers., Syn. PI. 267. 1805. C. thevetia L., Sp. PI. 209. 1753. Cascabela thevetia (L.) Lippold, Feddes Repert. 91: 52. 1980; Maliya \& Datt, J. Econ. Taxon. Bot. 34 (1): 56. 2010.

Note: Sometimes this species is placed in the genus Cascabela.

Habit: Evergreen shrub or small tree, cultivated.

Specimens examined: 21.v.1987, K.K. Singh \& party 5980 (LWG); 09.xi.2002, road side, Katerniaghat range, coll. S.D. Maliya 224561 (LWG); 24.iv.2003, Murtiha range, coll. S.D. Maliya 214832 (LWG); 16.iii.2008, Murtiha range, coll. S.D. Maliya 218515 (LWG).

**Tylophora himalaica Hook. f. in Hook. f., Fl. Brit. India 4: 42. 1883; Maliya \& Datt, J. Econ. Taxon. Bot. 34 (1): 57.2010.

Habit: Twining undershrub.

Specimens examined: 20.iv.2003, road side, Murtiha range, coll. S.D. Maliya 224953 (LWG).

Vallaris solanacea (Roth) Kuntz., Rev. Gen. 2: 417. 1891; Panigrahi et al., Bull. Bot. Surv. India 11 (1 \& 2): 101. 
1969.

Peltanthera solanacea Roth., Nov. PI. Sp. 132. 1821. Vallaris heynei Spreng., Syst. Veg. 1: 635. 1824; Hook. f. in Hook. f., Fl. Brit. India 3: 650. 1882; Duthie, FI. Upp. Gang. Plain 1: 488. repr. ed. 1960.

Habit: Twining shrub.

Specimens examined: 15.iii.1964, Rampurwa, G. Panigrahi 2907 (BSA); 20.xi.1964, Nishangara range, G. Panigrahi 6494 (BSA); 13.ii.1965, Nishangara range, O.P. Mishra 7967 (BSA) (Image 6).

Wrightia arborea (Dennst.) Mabberley, Taxon 26: 533. 1977; Maliya \& Datt, J. Econ. Taxon. Bot. 34 (1): 56. 2010.

Periploca arborea Dennst., Schlussel Hort. Malab. 13, 23, 35. 1818. Wrightia tomentosa (Roxb.) Roem. \& Schult., Syst. Veg. 4: 414. 1819. Nerium tomentosum Roxb., FI. ind. 2:6. 1824 (Hort. bengal. 84. 1814, nom. nud.).

Habit: Small deciduous tree.

Specimens examined: 20.xi.1964, Nishangara range, G. Panigrahi 6488 (BSA); 13.ii.1965, Nishangara range, O.P. Mishra 7966 (BSA); 13.xi.2002, Murtiha range, coll. S.D. Maliya 224667 (LWG); 21.xii.2003, Katerniaghat range, Katerniaghat Wildlife Sanctuary, coll. S.D. Maliya 225225 (LWG).

\section{ARISTOLOCHIACEAE}

Aristolochia indica L., Sp. PI. 960. 1753; Hook. f. in Hook. f., Fl. Brit. India 5: 75. 1886; Duthie, Fl. Upp. Gang. Plain 3: 161. repr. ed. 1960; Panigrahi et al., Bull. Bot.

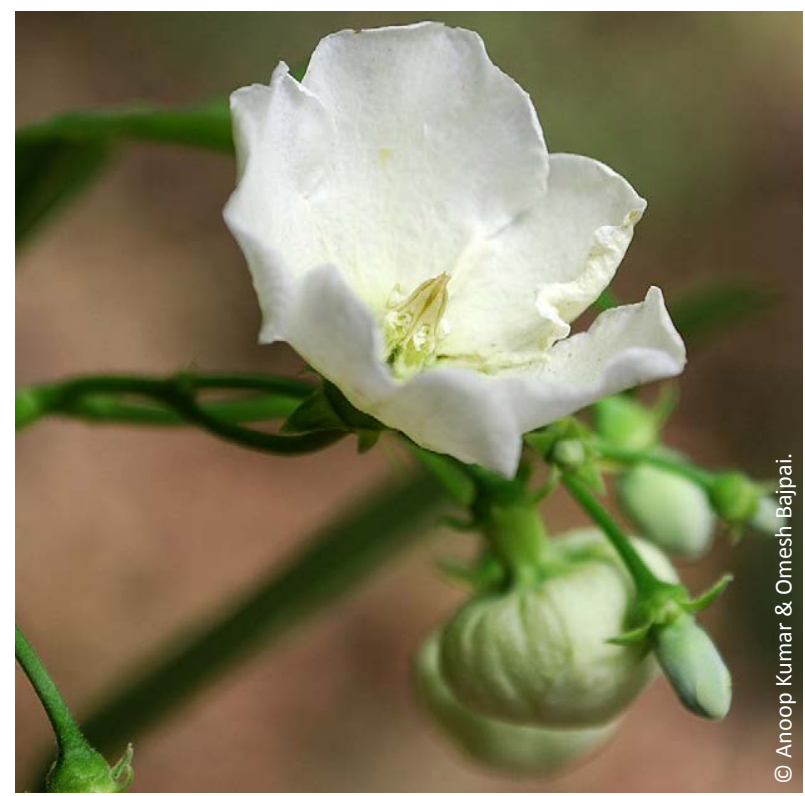

Image 6. Vallaris solanacea (Roth) Kuntz.
Surv. India 11 (1 \& 2): 103. 1969.

A. lanceolata Wight, Ic. PI. Ind. Orient. 5: t. 1858. 1852.

Habit: Climbing perennial herb.

Specimens examined: Not observed. Record based on Panigrahi et al. (1969).

\section{Asteraceae}

Acmella calva (DC.) Jansen, Syst. Bot. Monogr. 8: 41. 1985.

Spilanthes calva DC. in Wight., Contr. Bot. India 19. 1834; Maliya \& Datt, J. Econ. Taxon. Bot. 34 (1): 55. 2010. S. acmella var. calva (DC.) Clarke ex Hook. f. in Hook. f., Fl. Brit. India 3: 307. 1881; Gamble, Fl. Pres. Madras 709. 1921.

Habit: Annual herb.

Specimens examined: 23.viii.2002, Nishangara range, coll. S.D. Maliya 223640 (LWG); 09.xi.2002, near Bichhiya town, railway track, coll. S.D. Maliya 224513 (LWG); 12.xi.2002, Nishangara range, coll. S.D. Maliya 224652 (LWG); 13.xi.2002, Nishangara range, coll. S.D. Maliya 224674 (LWG); 14.xi.2002, Murtiha range, coll. S.D. Maliya 224672 (LWG); 31.viii.2003, Fakirpur village, coll. S.D. Maliya 214885 (LWG).

Adenostemma lavenia (L.) Kuntze, Revis. Gen. PI. 1: 304. 1891; Maliya \& Datt, J. Econ. Taxon. Bot. 34 (1): 55. 2010.

Verbesina lavenia L., Sp. PI. 902. 1753.

Habit: Annul herb.

Specimens examined: 12.xi.2002, Nishangara range, coll. S.D. Maliya 224623 (LWG).

Ageratum conyzoides L., Sp. PI. 839. 1753; Hook. f. in Hook. f., Fl. Brit. India 3: 243. 1881; Duthie, Fl. Upp. Gang. Plain 1: 405. repr. ed. 1960; Panigrahi et al., Bull. Bot. Surv. India 11 (1 \& 2): 105. 1969; Saini, J. Econ. Taxon. Bot. 29 (3): 594. 2005; Maliya \& Datt, J. Econ. Taxon. Bot. 34 (1): 55.2010.

Habit: Annual herb.

Specimens examined: 16.iii.2008, Murtiha range, coll. S.D. Maliya 218512 (LWG).

**Artemisia indica Willd., Sp. PI. 3: 1846. 1800; Maliya \& Datt, J. Econ. Taxon. Bot. 34 (1): 55. 2010.

Habit: Aromatic perennial herb.

Specimens examined: 22.x.2004, Kuthiyaghat forest, coll. S.D. Maliya 225996 (LWG); 26.x.2010, Kuthiyaghat forest, coll. S.D. Maliya 252637 (LWG).

Artemisia nilagirica (Clarke) Pamp., Nuovo Giorn. Bot. Ital., n.s., 33(3): 452. 1926; Maliya \& Datt, J. Econ. Taxon. 
Bot. 34 (1): 55. 2010.

A. vulgaris var. nilagirica Clarke, Compos. Ind. 162. 1876.

Habit: Perennial herb.

Specimens examined: 27.xii.2005, village Lahaura, Nishangara range, coll. S.D. Maliya 227191 (LWG).

**Ayapana triplinervis (Vahl) R. M. King \& H. Rob., Phytologia 20: 212. 1970.

Eupatorium triplinerve Vahl, Symb. Bot. 3: 97. 1794; Maliya \& Datt, J. Econ. Taxon. Bot. 34 (1): 55. 2010.

Habit: Herb.

Specimens examined: 09.xi.2002, road side, Katerniaghat range, coll. S.D. Maliya 224552 (LWG).

Blumea axillaris (Lam.) DC., Prodr. 5: 434. 1836.

Conyza axillaris Lam., Encycl. 2(1): 84. 1786. Erigeron mollis D.Don, Prodr. Fl. Nepal. 172. 1825. Blumea mollis (D.Don) Merr., Philipp. J. Sci. 5(5): 395. 1910.

Habit: Herb.

Specimens examined: 26.v.1954, Motipur range, Hira Lal 13429 (LWG) (Image 7).

Blumea eriantha DC. in Wight, Contrib. Bot. Ind. 15. 1834; Hook. f. in Hook. f., Fl. Brit. India 3: 266. 1881; Duthie, Fl. Upp. Gang. Plain 1: 413. repr. ed. 1960; Saini, J. Econ. Taxon. Bot. 29 (3): 595. 2005.

Habit: Herb.

Specimens examined: Not observed. Record based on Saini (2005)

Blumea fistulosa (Roxb.) Kurz, J. Asiat. Soc. Bengal 46 (2): 187. 1877; Randeria, Blumea 10: 256. 1960; Saini, J. Econ. Taxon. Bot. 29 (3): 595. 2005.

Conyza fistulosa Roxb., Fl. Ind. 3: 429. 1832.

Habit: Herb.

Specimens examined: Not observed. Record based on Saini (2005).

Blumea membranacea DC., Prodr. 5: 440. 1836; Hook. f. in Hook. f., Fl. Brit. India 3: 265. 1881; Saini, J. Econ. Taxon. Bot. 29 (3): 596. 2005.

Habit: Annual herb.

Specimens examined: 26.v.1954, Motipur farm, Hira Lal 13421 (LWG) (Image 8).

Blumea obliqua (L.) Druce, Rep. Bot. Exch. Club 1916: 609. 1917; Panigrahi et al., Bull. Bot. Surv. India 11 (1 \& 2): 105.1969.

Erigeron obliquus L., Mant. PI. 573. 1771.

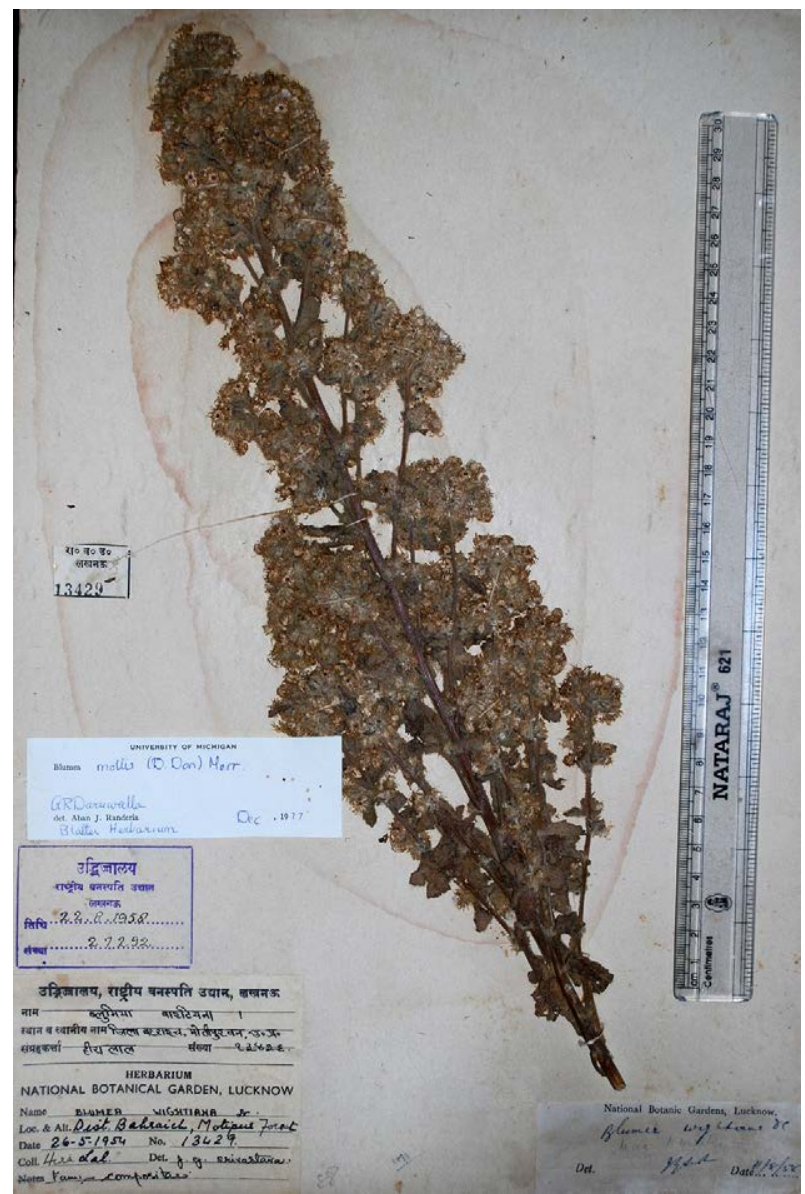

Image 7. Blumea axillaris (Lam.) DC.

Habit: Herb.

Specimens examined: 09.ii.1965, Jairampurwa, Motipur range, O.P. Mishra 7767 (BSA) (Image 9).

Blumeopsis falcata (D. Don) Merr., J. Arn. Arb. 19: 70. 1938; Panigrahi et al., Bull. Bot. Surv. India 11 (1 \& 2): 105. 1969.

Erigeron falcatum D. Don, Prodr. Fl. Nepal. 172. 1825. Conyza leucantha (D. Don) Ludlow \& P. H. Raven, Kew Bull. 17: 71. 1963.

Note: Sometimes (Global Compositae Checklist list) this species is treated under the genus Conyza.

Habit: Herb.

Specimens examined: 10.ii.1965, Nishangara range, O.P. Mishra 7908 (BSA) (Image 10).

Caesulia axillaris Roxb., Pl. Coromandel 1: 64, t. 93. 1795; Maliya, J. Econ. Taxon. Bot. 36 (1): 158. 2012 \& 36 (2): 421.2012.

Habit: Semiaquatic herb.

Specimens examined: 27.x.2010, Fakirpuri village, 


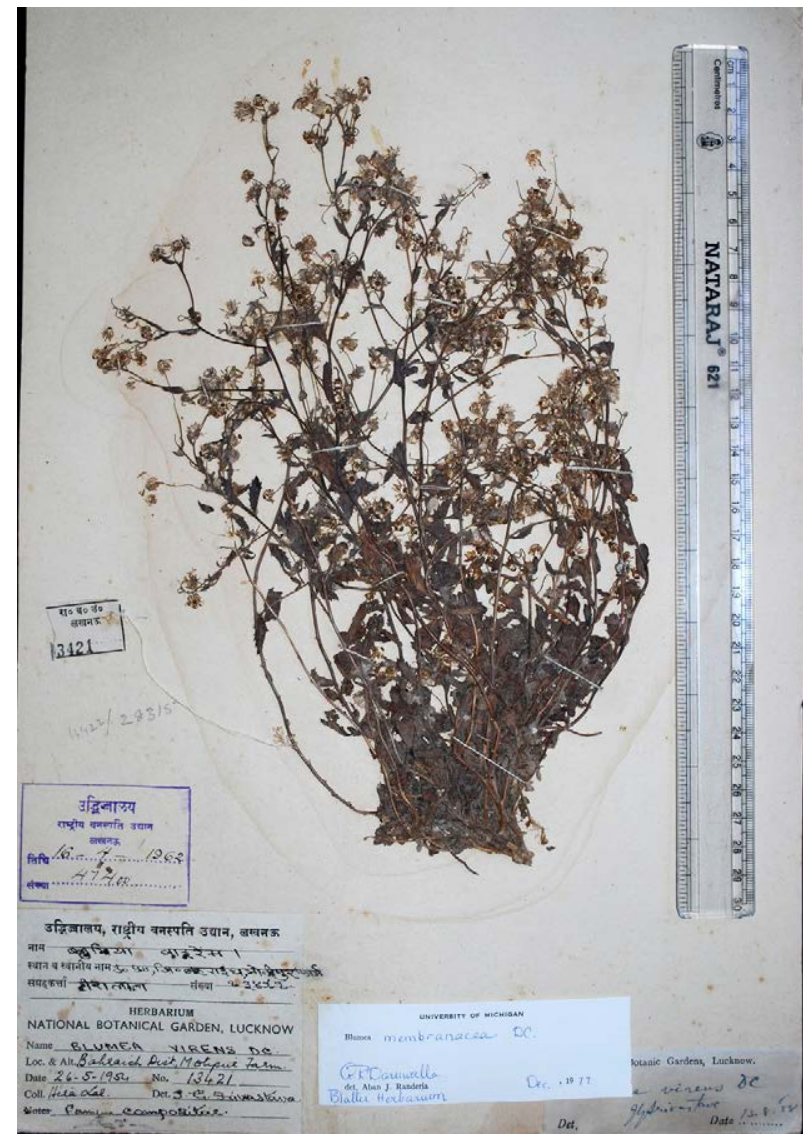

Image 8. Blumea membranacea DC.

near Rampurwa forest, Nishangara range, coll. S.D. Maliya 252639 (LWG).

**Cirsium arvense (L.) Scop., Fl. Carn. (ed. 2) 2: 126. 1772; Saini, J. Econ. Taxon. Bot. 29 (3): 597. 2005.

Serratula arvensis L., Sp. PI. 820. 1753. Cnicus arvensis Hoffm., Deutschl. Fl. 2: 130. 1804; Hook. f. in Hook. f., Fl. Brit. India 3: 362. 1881; Duthie, Fl. Upp. Gang. Plain 1: 438. repr. ed. 1960.

Habit: Erect herb.

Specimens examined: 12.iii.1964, Murtiha gate, G. Panigrahi 2868 (LWG).

**Cissampelopsis corifolia C. Jeffrey \& Y. L. Chen, Kew Bull. 39(2): 342, f. 21. 1984; Maliya, J. Econ. Taxon. Bot. 36 (2): 421. 2012.

Habit: Climber.

Specimens examined: Not observed. Record based on Maliya (2012).

**Conyza aegyptiaca (L.) Dryand. in Ait., Hort. Kew. 3: 183. 1789; Duthie, Fl. Upp. Gang. Plain 1: 409. repr. ed. 1960; Saini, J. Econ. Taxon. Bot. 29 (3): 597. 2005; Maliya

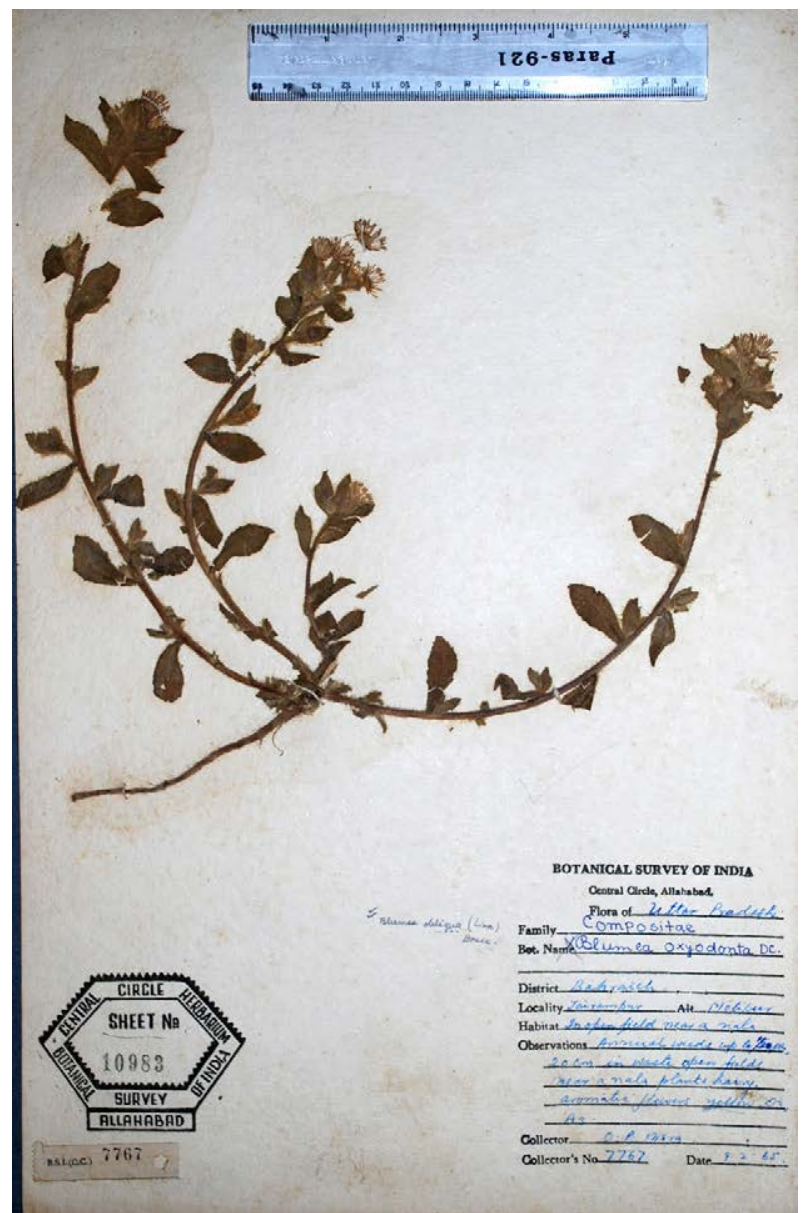

Image 9. Blumea obliqua (L.) Druce

\& Datt, J. Econ. Taxon. Bot. 34 (1): 55. 2010.

Erigeron aegyptiacus L., Mant. PI. 112. 1767.

Habit: Annual herb.

Specimens examined: 25.viii.2002, Murtiha range, coll. S.D. Maliya 223711 (LWG).

Conyza japonica Less., Syn. Campos. 204. 1832; Hook. f. in Hook. f., Fl. Brit. India 3: 258. 1881; Saini, J. Econ. Taxon. Bot. 29 (3): 598. 2005.

Habit: Annual herb.

Specimens examined: Not observed. Record based on Saini (2005).

Cotula anthemoides L., Sp. PI. 891. 1753; Hook. f. in Hook. f., Fl. Brit. India 3: 316. 1881; Duthie, Fl. Upp. Gang. Plain 1: 434. repr. ed. 1960; Panigrahi et al., Bull. Bot. Surv. India 11 (1 \& 2): 105. 1969; Saini, J. Econ. Taxon. Bot. 29(3): 598. 2005.

Habit: Annual herb.

Specimens examined: 12.iii.1964, Murtiha gate, G. Panigrahi 2883 (LWG). 


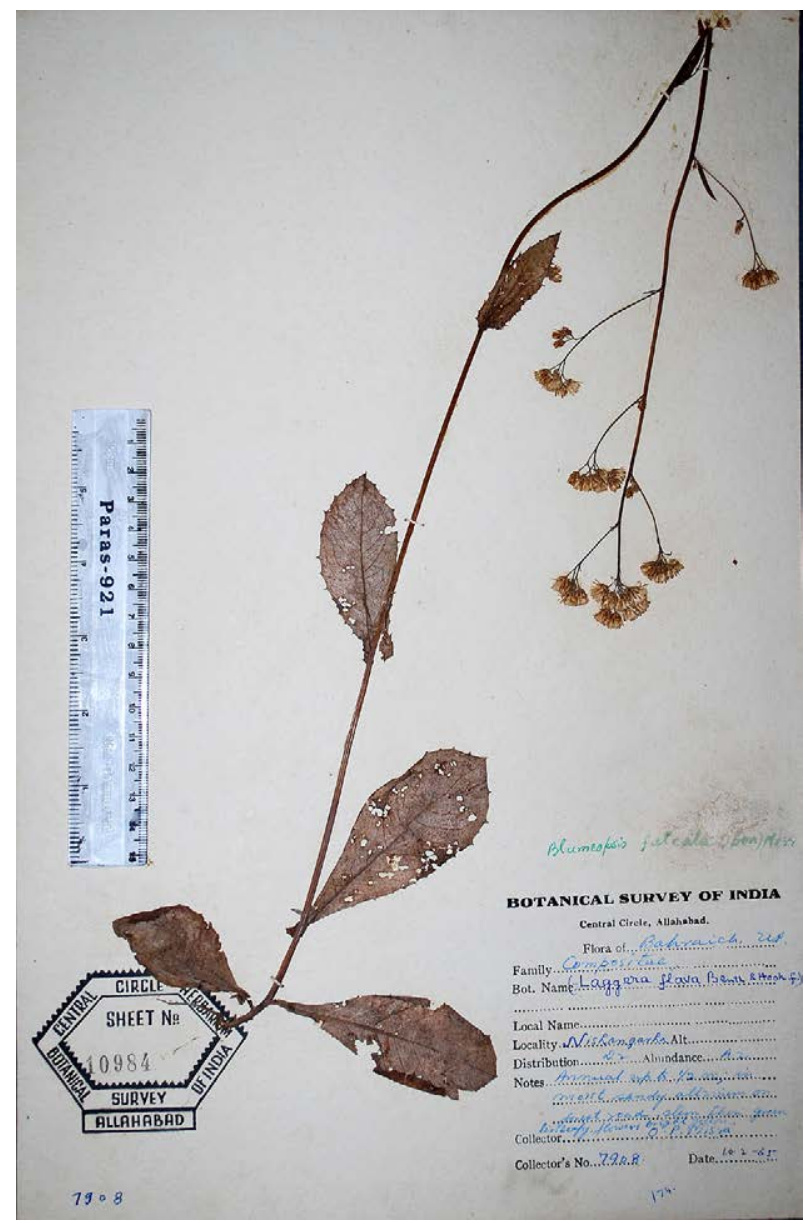

Image 10. Blumeopsis falcata (D. Don) Merr.

Cyanthillium cinereum (L.) H. Rob., Proc. Biol. Soc. Washington 103: 252. 1990.

Conyza cinerea L., Sp. PI. 862. 1753. Vernonia cinerea (L.) Less, Linnaea 4: 291. 1829; Hook. f. in Hook. f., Fl. Brit. India 3: 233. 1881; Duthie, Fl. Upp. Gang. Plain 1: 404. repr. ed. 1960; Maliya \& Datt, J. Econ. Taxon. Bot. 34 (1): 55. 2010.

Habit: Herb.

Specimens examined: 09.xi.2002, near Bichhiya town, coll. S.D. Maliya 224512 (LWG); 24.xii.2003, Kuthiyaghat forest, coll. S.D. Maliya 225258 (LWG).

Cyathocline purpurea (Buch.-Ham. ex D. Don) Kuntze, Revis. Gen. PI. 1:333. 1891; Maliya, J. Econ. Taxon. Bot. 36 (1): 158. 2012 \& 36 (2): 422. 2012.

Tanacetum purpureum Buch.-Ham. ex D. Don, Prodr. FI. Nepal. 181. 1825.

Habit: Annual herb.

Specimens examined: 15.iii.2010, Rampurwa forest, coll. S.D. Maliya 252614 (LWG).
Echinops echinatus Roxb., Fl. Ind. 447. 1832; Duthie, Fl. Upp. Gang. Plain 1: 437. repr. ed. 1960; Maliya \& Datt, J. Econ. Taxon. Bot. 34 (1): 55. 2010.

Habit: Annual herb.

Specimens examined: 17.iv.2003, Katerniaghat range, coll. S.D. Maliya 224913 (LWG); 22.iv.2003, Nishangara range, coll. S.D. Maliya 224970 (LWG).

Eclipta prostrata (L.) L., Mant. PI. 2: 286. 1771; Saini, J. Econ. Taxon. Bot. 29 (3): 598. 2005; Maliya \& Datt, J. Econ. Taxon. Bot. 34 (1): 55. 2010.

Verbesina prostrata L., Sp. PI. 902. 1753. Eclipta erecta L., Mant. PI. 2: 286. 1771; Duthie, Fl. Upp. Gang. Plain 1: 427. repr. ed. 1960.

Habit: Herb.

Specimens examined: 25.viii.2002, rest house, Murtiha range, coll. S.D. Maliya 223745 (LWG).

Elephantopus scaber L., Sp. PI. 814. 1753; Hook. f. in Hook. f., Fl. Brit. India 3: 242. 1881; Maliya \& Datt, J. Econ. Taxon. Bot. 34 (1): 55. 2010.

Habit: Herb.

Specimens examined: 23.viii.2002, Nishangara range, coll. S.D. Maliya 223609 (LWG); 10.xi.2002, Murtiha range, coll. S.D. Maliya 224588 (LWG); 16.vii.2004, Kuthiyaghat forest, coll. S.D. Maliya 225946 (LWG); 07.x.2009, Kakraha range, coll. S.D. Maliya 226652 (LWG).

Emilia sonchifolia (L.) DC. in Wight., Contr. Bot. India 24. 1834; Hook. f. in Hook. f., Fl. Brit. India 3: 336. 1881; Saini, J. Econ. Taxon. Bot. 29 (3): 599. 2005

Cacalia sonchifolia L., Sp. PI. 835. 1753.

Habit: Annual herb.

Specimens examined: Not observed. Record based on Saini (2005).

**Enhydra fluctuans Lour., Fl. Cochinch. 2: 511. 1790; Hook. f. in Hook. f., Fl. Brit. India 3: 304. 1881; Raizada, Suppl. Fl. Upp. Gang. Plain 111. 1976; Saini, J. Econ. Taxon. Bot. 29 (3): 599. 2005; Maliya \& Datt, J. Econ. Taxon. Bot. 34 (1): 55.2010.

Habit: Floating or marshy herb.

Specimens examined: 22.iv.2003, Nishangara range, coll. S.D. Maliya 224991 (LWG).

**Gnaphalium polycaulon Pers., Syn. PI. 2(2): 421. 1807 "policaulon"; Maliya, J. Econ. Taxon. Bot. 36 (2): 422. 2012.

Habit: Herb.

Specimens examined: 08.iii.2010, near urai nala, Nishangara range, coll. S.D. Maliya 226693 (LWG). 
Note: In some cases Blumea obliqa (L.) Druce is as synonym of the same.

**Gamochaeta purpurea (L.) Cabrera, Bol. Soc. Argent. Bot. 9: 377. 1961.

Gnaphalium purpureum L., Sp. PI. 854. 1753. G. pensylvanicum Willd., Enum. Hort. Berd. 867. 1809; Saini, J. Econ. Taxon. Bot. 29 (3): 599. 2005. Gamochaeta pensylvanica (Willd.) Cabrera, Bol. Soc. Argent. Bot. 9: 375. 1961.

Habit: Herb.

Specimens examined: Not observed. Record based on Saini (2005)

Note: Sometimes Gamochaeta pensy/vanica (Willd.) Cabrera is erroneously accepted as correct name.

Gnomophalium pulvinatum (Delile) Greuter, Willdenowia 33 (2): 242. 2003.

Gnaphalium pulvinatum Delile, Descr. Egypte, Hist. Nat. Tom. Second 122, t. 44, f. 1. 1812; Hook. f. in Hook. f., Fl. Brit. India 3: 289. 1881; Panigrahi et al., Bull. Bot. Surv. India 11 (1 \& 2): 105. 1969; Saini, J. Econ. Taxon. Bot. 29 (3): 600. 2005.

Habit: Annual herb.

Specimens examined: 12.iii.1964, Murtiha gate, G. Panigrahi 2882 (LWG).

Ixeris polycephala Cass. in Cuvier, Dict. Sci. Nat. 24: 50. 1822.

Lactuca polycephala (Cass.) Benth., Gen. PI. 2: 526. 1873; Panigrahi et al., Bull. Bot. Surv. India 11 (1 \& 2): 105. 1969.

Habit: Annual herb.

Specimens examined: 14.ii.1965, Urai tal, Bichhiya, G. Panigrahi 7989 (LWG).

Lactuca dissecta D. Don, Prodr. fl. Nepal. 164. 1825. Habit: Annual herb.

Specimens examined: 14.iii.2010, Rampurwa rest house, Nishangara range, coll. S.D. Maliya 252612 (LWG).

**Laggera aurita (L. f.) Benth. ex Clarke, Compos. Ind. 92. 1876; Hook. f. in Hook. f., Fl. Brit. India 3: 271. 1881; Duthie, FI. Upp. Gang. Plain 1: 417. repr. ed. 1960; Saini, J. Econ. Taxon. Bot. 29 (3): 600. 2005.

Conyza aurita L. f., Sp. PI. 367. 1782.

Habit: Perennial herb.

Specimens examined: Not observed. Record based on Saini (2005)
**Laphangium luteoalbum (L.) Tzvelev, Byull. Moskovsk. Obshch. Isp. Prir. Otd. Biol. 98(6): 105. 1994.

Gnaphalium luteoalbum L., Sp. PI. 851. 1753. G. multiceps Wall. ex DC., Prodr. 6: 222. 1838. G. luteoalbum L. var. multiceps (Wall. ex DC.) Hook. f. in Hook. f., FI. Brit. India 3: 288. 1881; Duthie, Fl. Upp. Gang. Plain 1: 288. repr. ed. 1960; Panigrahi et al., Bull. Bot. Surv. India 11 (1 \& 2): 105. 1969. Gnaphalium luteoalbum L. var. pallidum Hook. f. in Hook. f., Fl. Brit. India 3: 288. 1881; Duthie, Fl. Upp. Gang. Plain 1: 421. repr. ed. 1960; Panigrahi et al., Bull. Bot. Surv. India 11 (1 \& 2): 105. 1969; Saini, J. Econ. Taxon. Bot. 29 (3): 599. 2005. Pseudognaphalium luteoalbum (L.) Hilliard \& B. L. Burtt, Bot. J. Linn. Soc. 82 (3): 206. 1981.

Habit: Annual herb.

Specimens examined: 14.ii.1965, Bichhhiya, Nishangara range, O.P. Mishra 7982 (BSA).

Launaea acaulis (Roxb.) Babc. ex Kerr. in Craib, Fl. Siam. Enum. 2: 299. 1936; Saini, J. Econ. Taxon. Bot. 29 (3): 599. 2005; Maliya \& Datt, J. Econ. Taxon. Bot. 34 (1): 55. 2010

Prenanthes acaulis Roxb., FI. Ind. 3: 403. 1832. Crepis acaulis (Roxb.) Hook. f. in Hook. f., Fl. Brit. India 3: 396. 1881.

Habit: Perennial herb.

Specimens examined: 22.iv.2003, Nishangara range, coll. S.D. Maliya 224997 (LWG).

Launaea aspleniifolia (Willd.) Hook. f. in Hook. f., Fl. Brit. India 3: 415. 1881; Maliya \& Datt, J. Econ. Taxon. Bot. 34 (1): 55. 2010.

Prenanthes asplenifolia Willd., Sp. PI. 3(3): 1540. 1803.

Habit: Herb.

Specimens examined: 17.iii.2004, Kuthiyaghat forest, coll. S.D. Maliya 225903 (LWG); 09.iii.2010, Katerniaghat range, coll. S.D. Maliya 226699 (LWG).

Launaea procumbens (Roxb.) Ramaya \& Rajgopal, Kew Bull. 23: 465. 1969; Saini, J. Econ. Taxon. Bot. 29 (3): 600. 2005; Maliya \& Datt, J. Econ. Taxon. Bot. 34 (1): 55. 2010.

Prenanthes procumbens Roxb., FI. Ind. 3: 404. 1832. Microrynchus fallax J. \& S., III. PI. Orient. 3: 106. 1848. Launaea fallax (J. \& S.) Kuntze, Rev. Gen. PI. 1: 350. 1891; Panigrahi et al., Bull. Bot. Surv. India 11 (1 \& 2): 105. 1969. Habit: Herb.

Specimens examined: 23.iv.2003, Kuthiyaghat forest, coll. S.D. Maliya 225941 (LWG). 
Oligochaeta ramosa (Roxb.) Wagenitz, Veroff. Geobot. Inst. ETH Stiftung Rubel Zurich 37: 323. 1962.

Carduus ramosus Roxb., Fl. Ind. 3: 407. 1832. Volutarella divaricata Benth. \& Hook. f., Gen. PI. 2: 476. 1873 pp. \& in Hook. f., Fl. Brit. India 3: 383. 1881; Duthie, Fl. Upp. Gang. Plain 1: 442. repr. ed. 1960. Amberboa ramosa (Roxb.) Jafri, Scientist 3: 29. 1959; Panigrahi et al., Bull. Bot. Surv. India 11 (1 \& 2): 105. 1969; Saini, J. Econ. Taxon. Bot. 29 (3): 594. 2005. Volutarella ramosa (Roxb.) Sant., Fl. Saur. 22. 1963.

Habit: Annual herb.

Specimens examined: Not observed. Record based on Panigrahi et al. (1969) and Saini (2005).

**Parthenium hysterophorus L., Sp. PI. 988. 1753; Raizada, Suppl. FI. Upp. Gang. Plain 127. 1976; Maliya \& Datt, J. Econ. Taxon. Bot. 34 (1): 55. 2010.

Habit: Herb.

Specimens examined: 13.xi.2011. near Geruwa river, Katerniaghat range, A. Kumar \& O. Bajpai 252789 (LWG).

Pentanema indicum (L.) Y. Ling, Acta Phytotax. Sin. 10: 179. 1965.

Inula indica L., Sp. pl. 1236. 1763. Vicoa indica (L.) DC. in Wight, Contr. bot. India 10. 1834; Panigrahi et al., Bull. Bot. Surv. India 11 (1 \& 2): 106. 1969.

Habit: Herb.

Specimens examined: 13.ii.1965, Nishangara range, O.P. Mishra 7976 (BSA).

Pentanema vestitum (Wall. ex DC.) Ling, Acta Phytotax. Sin. 10(2): 180. 1965; Saini, J. Econ. Taxon. Bot. 29 (3): 601. 2005; Maliya \& Datt, J. Econ. Taxon. Bot. 34 (1): 55. 2010.

Inula vestita Wall. ex DC., Prodr. 5: 470. 1836. Vicoa vestita (Wall. ex DC.) Benth. ex Hook. f. in Hook. f., Fl. Brit. India 3: 297. 1881.

Habit: Herb.

Specimens examined: 26.v.1954, Motipur range, Hira Lal 13423 (LWG); 20.iv.2003, Murtiha range, coll. S.D. Maliya 224955 (LWG); 18.iii.2004, Kuthiyaghat forest, coll. S.D. Maliya 225914 (LWG); 20.iii.2008, Lahauri village, Nishangara range, coll. S.D. Maliya 218577 (LWG).

Pseudognaphalium affine (D. Don) Anderb., Opera Bot. 104: 146. 1991.

Gnaphalium affine D. Don., Prodr. Fl. Nepal 173. 1825. G. luteo-album L. ssp. affine (D. Don) Koster, Blumea 4 (3): 484. 1941; Saini, J. Econ. Taxon. Bot. 29 (3): 599. 2005; Maliya \& Datt, J. Econ. Taxon. Bot. 34 (1): 55. 2010.

Habit: Annual herb.
Specimens examined: 17.iv.2003, Katerniaghat range, coll. S.D. Maliya 224914 (LWG).

Pulicaria angustifolia DC., Prodr. 5: 479. 1836; Duthie, Fl. Upp. Gang. Plain 1: 425. repr. ed. 1960; Panigrahi et al., Bull. Bot. Surv. India 11 (1 \& 2): 105. 1969.

Habit: Herb.

Specimens examined: 13.ii.1965, Nishangara range, O.P. Mishra 7973 (LWG).

** Sigesbeckia orientalis L., Sp. PI. 900. 1753; Hook. f. in Hook. f., Fl. Brit. India 3: 304. 1881; Panigrahi et al., Bull. Bot. Surv. India 11 (1 \& 2): 105. 1969; Saini, J. Econ. Taxon. Bot. 29 (3): 602. 2005; Maliya \& Datt, J. Econ. Taxon. Bot. 34 (1): 55. 2010.

S. brachiata Roxb., FI. Ind. 3: 439. 1832.

Habit: Annual herb.

Specimens examined: 09.xi.2002, road side, Katerniaghat range, coll. S.D. Maliya 224564 (LWG); 23.xii.2003, Girijapuri forest, coll. S.D. Maliya 225243 (LWG).

*Solidago canadensis L., Sp. Pl. 878. 1753; Saini, J. Econ. Taxon. Bot. 29 (3): 602. 2005.

Habit: Perennial herb, Cultivated.

Specimens examined: Not observed. Record based on Saini (2005).

**Soliva anthemifolia (Juss.) Sweet, Hort. Brit. 243. 1826; Raizada, Suppl. Fl. Upp. Gang. Plain 133. 1976; Saini, J. Econ. Taxon. Bot. 29 (3): 602. 2005.

Gymnostyles anthemifolia Juss., Ann. Mus. Natl. Hist. Nat. 4: 262, t. 61, f. 1. 1804.

Habit: Annual herb.

Specimens examined: Not observed. Record based on Saini (2005).

**Sonchus asper (L.) Hill, Herb. Brit. 1: 47. 1769; Jeffrey, Kew Bull. 18: 481. 1966.

S. oleraceus var. asper L., Sp. PI. 794. 1753; Saini, J. Econ. Taxon. Bot. 29 (3): 602. 2005.

Habit: Annual herb.

Specimens examined: 20.xi.1964, Murtiha gate, G. Panigrahi 2869 (BSA).

** Tridax procumbens L., Sp. PI. 900. 1753; Hook. f. in Hook. f., Fl. Brit. India 3: 311. 1881; Duthie, Fl. Upp. Gang. Plain 1: 433. repr. ed. 1960; Maliya \& Datt, J. Econ. Taxon. Bot. 34 (1): 55. 2010.

Habit: Straggling herb.

Specimens examined: 12.xii.1986, Motipur range, K.K. 
Khanna \& R. Saran 38664 (BSA).

Vernonia anthelmintica (L.) Willd., Sp. PI. 3(3): 1634 1803; Duthie, Fl. Upp. Gang. Plain 1: 402. repr. ed. 1960.

Conyza anthelmintica L., Sp. Pl. 1207. 1763. Centratherum anthelminticum (L.) Kuntze ex Gamble, Fl. Madras 667. 1929; Panigrahi et al., Bull. Bot. Surv. India 11 (1 \& 2): 105. 1969. Baccharoides anthelmintica (L.) Moench, Methodus 578. 1794.

Habit: Annual herb.

Specimens examined: 20.xi.1964, Nishangara range, G. Panigrahi \& O.P. Mishra 6449 (BSA) (Image 11).

Vernonia aspera (Roxb.) Buch.-Ham., Trans. Linn. Soc. London 14(2): 219. 1824.

Eupatorium asperum Roxb., Fl. Ind. 3: 415. 1832. Vernonia roxburghii Less., Linnaea 6: 674. 1831; Duthie, Fl. Upp. Gang. Plain 1: 402. repr. ed. 1960; Panigrahi et al., Bull. Bot. Surv. India 11 (1 \& 2): 106. 1969.

Habit: Herb or under shrub.

Specimens examined: Not observed. Record based on Panigrahi et al. (1969).

Vernonia divergens (DC.) Edg., J. Asiat. Soc. Bengal 21: 172. 1853; Hook. f. in Hook. f., Fl. Brit. India 3: 234. 1881; Duthie, Fl. Upp. Gang. Plain 1: 403. repr. ed. 1960; Saini, J. Econ. Taxon. Bot. 29 (3): 604. 2005.

Decaneurum divergens DC., Contr. Bot. India 8. 1834.

Habit: Perennial herb.

Specimens examined: Not observed. Record based on Saini (2005).

**Xanthium strumarium L., Sp. PI. 987. 1753; Hook. f. in Hook. f., Fl. Brit. India 3: 303. 1881; Duthie, FI. Upp. Gang. Plain 1: 426. repr. ed. 1960; Saini, J. Econ. Taxon. Bot. 29(3): 604. 2005.

X. indicum J. Koenig ex Roxb., Fl. Ind. 3: 601. 1832.

Habit: Herb.

Specimens examined: 12.iii.1964, Murtiha gate, G. Panigrahi 2861 (BSA).

**Youngia japonica (L.) DC., Prodr. 7: 194. 1838; Hara, Fl. East. Himal. 347. 1966; Maliya \& Datt, J. Econ. Taxon. Bot. 34 (1): 56. 2010.

Prenanthes japonica L., Mant. PI. 1: 107. 1767.

Habit: Herb.

Specimens examined: 17.iv.2003, road side, Katerniaghat range, coll. S.D. Maliya 224920 (LWG).

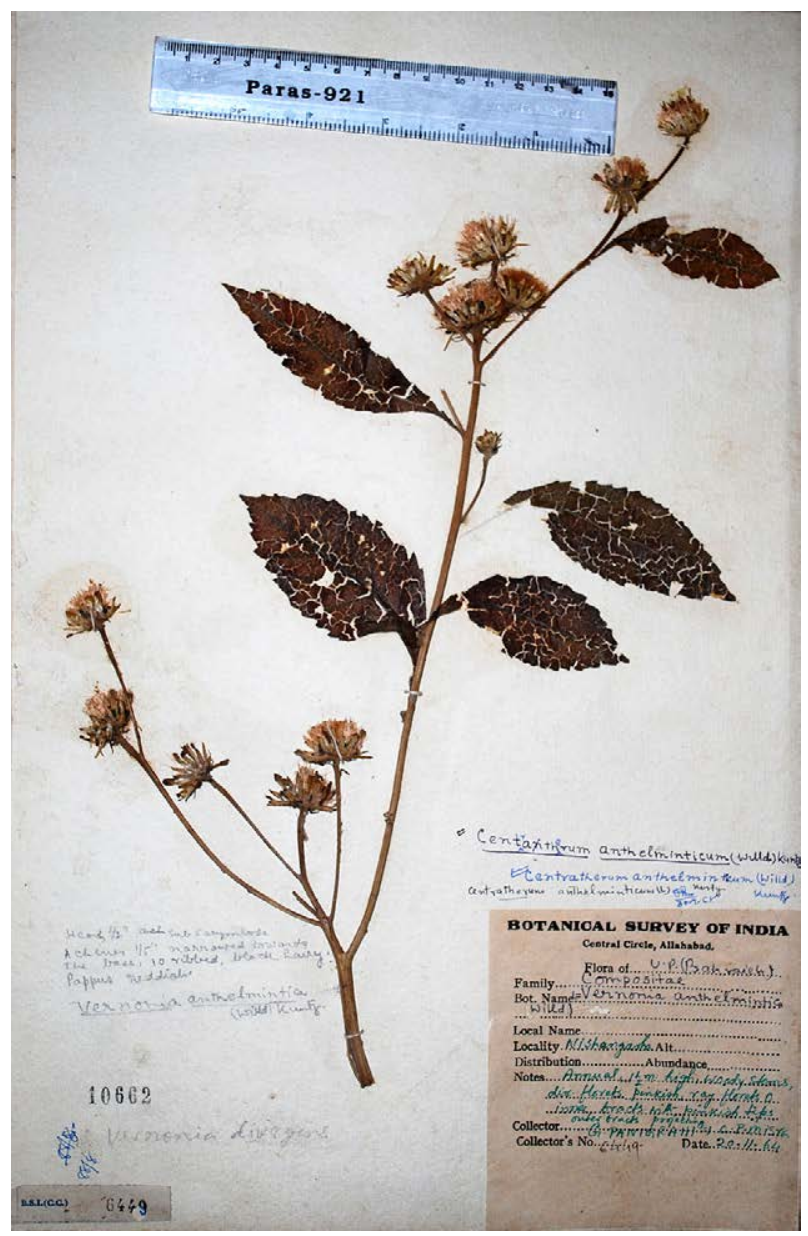

Image 11. Vernonia anthelmintica (L.) Willd.

\section{BALSAMINACEAE}

Impatiens balsamina L., Sp. PI. 938. 1753; Edg. \& Hook. f. in Hook. f., Fl. Brit. India 1: 453. 1874; Vivekananthan et al. in Sharma et al., Fl. India 4: 123. 1997; Maliya \& Datt, J. Econ. Taxon. Bot. 34 (1): 48. 2010.

Habit: Herb.

Specimens examined: 26.x.2004, Nepal border, Murtiha range, coll. S.D. Maliya 227116 (LWG).

\section{BASELLACEAE}

Basella alba L., Sp. PI. 272. 1753; Saini, J. Econ. Taxon. Bot. 29(4): 864. 2005.

B. rubra L., Sp. PI. 272. 1753; Hook. f. in Hook. f., Fl. Brit. India 5: 20. 1886; Duthie, Fl. Upp. Gang. Plain 3: 147. repr. ed. 1960; Panigrahi et al., Bull. Bot. Surv. India 11 (1 \& 2): 104. 1969; Maliya \& Datt, J. Econ. Taxon. Bot. 34 (1): 61. 2010.

Habit: Fleshy perennial climbing herb.

Specimens examined: 25.xi.1964, Plantation area, G. Panigrahi \& O.P. Mishra 6611A (BSA); 09.ii.1965, Motipur range, Katerniaghat Wildlife Sanctuary, Bharaich, O.P. 
Mishra 7776 (BSA).

\section{BIGNONIACEAE}

*Jacaranda mimosifolia D. Don, Bot. Reg. 8: 631, t. 631. 1822

J. chelonia Griseb. Abh. Konigl. Ges. Wiss. Gottingen 19: 223. 1874. J. ovalifolia R. Br., Bot. Mag. 49: t. 2327. 1822.

Habit: Tree, cultivated.

Specimens examined: 25.iv.2010, rest house, Nishangarha range, L.B. Chaudhary, A. Kumar \& O. Bajpai 252285 (LWG).

*Kigelia africana (Lam.) Benth., Hook. f. \& Benth. in Hook. Niger Fl. 463. 1849.

Bignonia africana Lam., Encycl. 1: 424. 1785.

Habit: Tree, cultivated.

Specimens examined: 04.iv.2011, Katerniaghat rest house, L.B. Chaudhary, A. Kumar \& O. Bajpai 263613 (LWG).

Oroxylum indicum (L.) Vent., Decas Gen. Nov. 8. 1808; Maliya \& Datt, J. Econ. Taxon. Bot. 34 (1): 59. 2010.

Bignonia indica L., Sp. PI. 625. 1753.

Habit: Tree.

Specimens examined: 31.viii.2003, Fakirpur village, coll. S.D. Maliya 214888 (LWG); 30.x.2009, right side of road of Katerniaghat to Bichhia road, between $5-7 \mathrm{Km}$. milestones L.B. Chaudhary, A. Kumar \& O. Bajpai 252147 (LWG).

Stereospermum chelonoides (L.f.) DC., Biblioth. Universelle Geneve 17: 124. 1838; Maliya \& Datt, J. Econ. Taxon. Bot. 34 (1): 59. 2010.

Bignonia chelonoides L. f., Suppl. PI. 282. 1782. B. suaveolens Roxb., Fl. Ind. 3: 104. 1832. Stereospermum suaveolens (Roxb.) DC., Biblioth. Universelle Geneve 17: 124. 1838; Panigrahi et al., Bull. Bot. Surv. India 11 (1 \& 2): 102. 1969.

Habit: Tree.

Specimens examined: 16.iv.1967, S. L. Kapoor \& party 70055 (LWG); 27.viii.2003, Katerniaghat range, coll. S.D. Maliya 214845 (LWG)

*Tecoma stans (L.) Juss. ex Kunth, Nov. Gen. Sp. 3: 144. 1819; Duthie, Fl. Upp. Gang. Plain 2: 42. repr. ed. 1960; Saini, J. Econ. Taxon. Bot. 29 (4): 845. 2005; Maliya \& Datt, J. Econ. Taxon. Bot. 34 (1): 59. 2010.

Begnonia stans L., Sp. PI. 871. 1763. Stenolobium stans (L.) Seem., J. Bot. 1: 88. 1863.

Habit: Small tree.
Specimens examined: 23.i.2005, road side, near inspection house, Girijapuri forest, coll. S.D. Maliya 227146 (LWG).

\section{BOMBACACEAE}

Bombax ceiba L., Sp. PI. 511. 1753 nom. cons.; Nayar \& Biswas in Sharma et al., Fl. India 3: 398. 1993; Bachulkar, Phytotax. 10: 143. 2010; Maliya \& Datt, J. Econ. Taxon. Bot. 34 (1): 48. 2010.

B. malabaricum DC., Prodr. 1: 479. 1824; Mast. in Hook. f., Fl. Brit. India 1: 349. 1874; Duthie, Fl. Upp. Gang. Plain 1: 92. repr. ed. 1960. Salmalia malabarica (DC.) Schott \& Endl., Melt. 35. 1832.

Habit: Tree.

Specimens examined: 27.viii.2003, road side, Katerniaghat range, coll. S.D. Maliya 214850 (LWG); 28.iv.2010, near Vardia chauki, Nishangara range, L.B. Chaudhary, A. Kumar \& O. Bajpai 252805 (LWG).

\section{BORAGINACEAE}

Arnebia hispidissima (Sieber ex Lehm.) DC., Prodr. 10: 94. 1846; Duthie, Fl. Upp. Gang. Plain 1: 541. repr. ed. 1960; Panigrahi et al., Bull. Bot. Surv. India 11 (1 \& 2): 108. 1969; Maliya \& Datt, J. Econ. Taxon. Bot. 34 (1): 57. 2010. Lithospermum hispidissimum Sieber ex Lehm., Ic. Nov. Stirp. 1: 23, t. 39. 1821.

Habit: Herb.

Specimens examined: 17.iv.2003, road side, Katerniaghat range, Murtiha range, coll. S.D. Maliya 224922 (LWG); 05.iii.2011, Katerniaghat dam, A. Kumar \& O. Bajpai 252870 (LWG); 04.iv.2011, Near Katerniaghat old railway station, Katerniaghat range, A. Kumar \& O. Bajpai 263628 (LWG) (Image 12).

Bothriospermum zeylanicum (Jacq.) Druce, Rep. Bot. Soc. Exch. Club Brit. Isles 4 (Suppl. 2): 610. 1917.

Anchusa zeylanica Jacq., Ecl. Pl. Rar. 1: 47, t. 29. 1812. A. tenella Hornem., Hort. Bot. Hafn. 1: 176. 1813. Bothriospermum tenellum (Hornem.) Fisch. \& Mey., Ind. Sem. Hort. Petrop. 1: 24. 1836; Clarke in Hook. f., Fl. Brit. India 4: 167. 1883; Duthie, Fl. Upp. Gang. Plain 1: 540. repr. ed. 1960; Panigrahi et al., Bull. Bot. Surv. India 11 (1 \& 2): 108.1969.

Habit: Herb.

Specimens examined: Not observed. Record based on Panigrahi et al. (1969).

Cordia dichotoma G. Forst., Fl. Ins. Austr. 18, no. 110. 1786; Maliya \& Datt, J. Econ. Taxon. Bot. 34 (1): 57. 2010. Habit: Tree.

Specimens examined: 02.ix.2003, Girijapuri forest, 


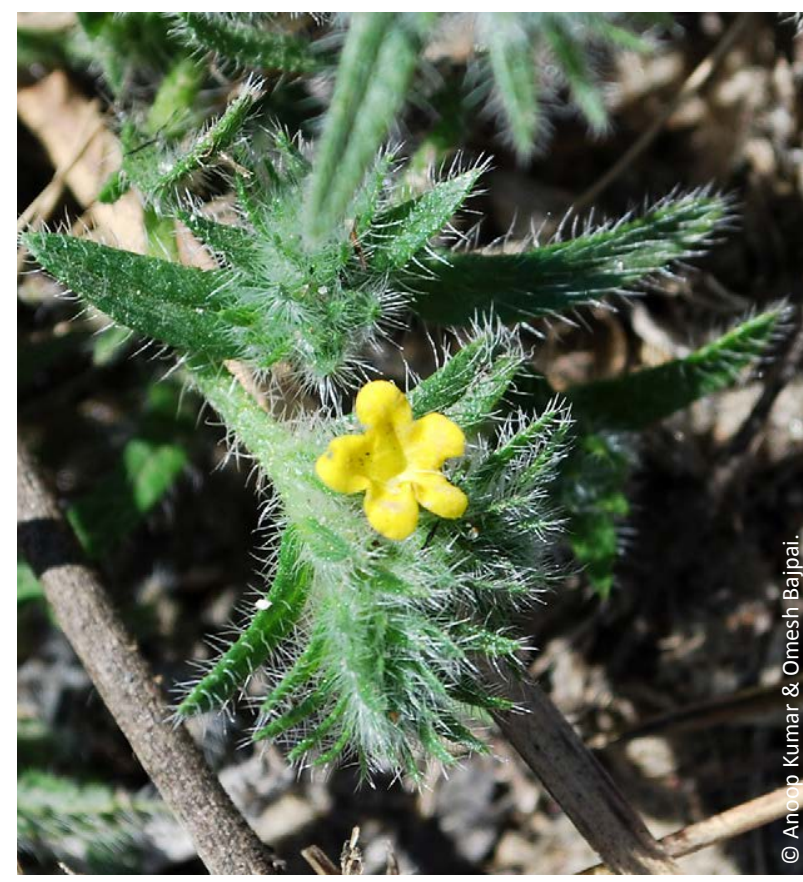

Image 12. Arnebia hispidissima (Sieber ex Lehm.) DC.

coll. S.D. Maliya 214896 (LWG).

Cordia grandis Roxb., FI. Ind. 2: 335. 1824; Panigrahi et al., Bull. Bot. Surv. India 11 (1 \& 2): 102. 1969.

Habit: Tree.

Specimens examined: Not observed. Record based on Panigrahi et al. (1969).

Cynoglossum lanceolatum Forssk., Fl. Aegypt. Arab. 41. 1775; Clarke in Hook. f., Fl. Brit. India 4: 156. 1883; Duthie, Fl. Upp. Gang. Plain 1: 540. repr. ed. 1960; Panigrahi et al., Bull. Bot. Surv. India 11 (1 \& 2): 108. 1969; Saini, J. Econ. Taxon. Bot. 29 (3): 618. 2005; Maliya \& Datt, J. Econ. Taxon. Bot. 34 (1): 57. 2010.

Cynoglossum micranthum Desf., Tabl. Ecole Bot. 1: 220. 1804.

Habit: Herb.

Specimens examined: 12.iii.1964, Motipur range, G. Panigrahi 2843 (BSA).

**Cynoglossum meeboldii Brand, Repert. Spec. Nov. Regni Veg.14: 323. 1916; Saini, J. Econ. Taxon. Bot. 29 (3): 619. 2005

Habit: Herb.

Specimens examined: Not observed. Record based on Saini (2005).

Cynoglossum zeylanicum (Vahl ex Hornem.) Thunb. ex Lehm., Neue Schriften Naturf. Ges. Halle 3(2): 20.
1817; Maliya \& Datt, J. Econ. Taxon. Bot. 34 (1): 57. 2010. Anchusa zeylanica Vahl ex Hornem., Hort. Bot. Hafn. 1: 176. 1813. Cynoglossum denticulatum DC. var. zeylanicum (Vahl ex Hornem.) Clark in Hook. f., Fl. Brit. India 4: 157. 1883; Duthie, Fl. Upp. Gang. Plain 1: 540. repr. ed. 1960; Saini, J. Econ. Taxon. Bot. 29 (3): 619. 2005.

Habit: Herb.

Specimens examined: 20.xi.1964, G. Panigrahi \& O.P. Mishra 6450 (BSA); 26.viii.2002, coll. S.D. Maliya 223769 (LWG); 13.xi.2002, Nishangara range, coll. S.D. Maliya 224664 (LWG).

Ehretia acuminata R. Br., Prodr. 1: 497. 1810.

Ehretia serrata Roxb., Fl. Ind. 2: 340. 1824. Ehretia acuminata var. serrata (Roxb.) Johnst., J. Arnold Arbor. 32: 23. 1951. Meliosma simplicifolia sensu Maliya \& Datt, J. Econ. Taxon. Bot. 34 (1): 50. 2010.

Habit: Tree.

Specimens examined: 21.iv.2003, Nishangara range, coll. S.D. Maliya 224958 (LWG).

Ehretia laevis Roxb., Pl. Coromandel 1: 42, t. 56. 1795; Clarke in Hook. f., Fl. Brit. India 4: 141. 1883; Duthie, Fl. Upp. Gang. Plain 1: 531. repr. ed. 1960.

Pittosporum napaulense sensu Maliya \& Datt, J. Econ. Taxon. Bot. 34 (1): 47. 2010.

Habit: Tree.

Specimens examined: .iv.1993, Motipur range, D.C. Saini 11519 (BSIP); 19.iv.2003, Murtiha range, coll. S.D. Maliya \& B. Datt 224936 (LWG); 21.iv.2003, Nishangara range, coll. S.D. Maliya 224957 (LWG); 09.iii.2010, roadside, Girijapuri forest, coll. S.D. Maliya 226700 (LWG) (Image 13).

Heliotropium marifolium J. Koenig ex Retz., Observ. Bot. 2: 8. 1781; Maliya \& Datt, J. Econ. Taxon. Bot. 34 (1): 57. 2010.

Habit: Procumbent perennial herb.

Specimens examined: 26.viii.2002, coll. S.D. Maliya 223776 (LWG).

Heliotropium ovalifolium Forssk., Fl. Aegypt.- Arab. 38. 1775; Clarke in Hook. f., Fl. Brit. India 4: 150. 1883; Duthie, Fl. Upp. Gang. Plain 1: 536. repr. ed. 1960; Saini, J. Econ. Taxon. Bot. 29 (3): 619. 2005.

H. coromadelianum Retz., Observ. Bot. 2: 9. 1781.

Habit: Herb.

Specimens examined: Not observed. Record based on Saini (2005). 


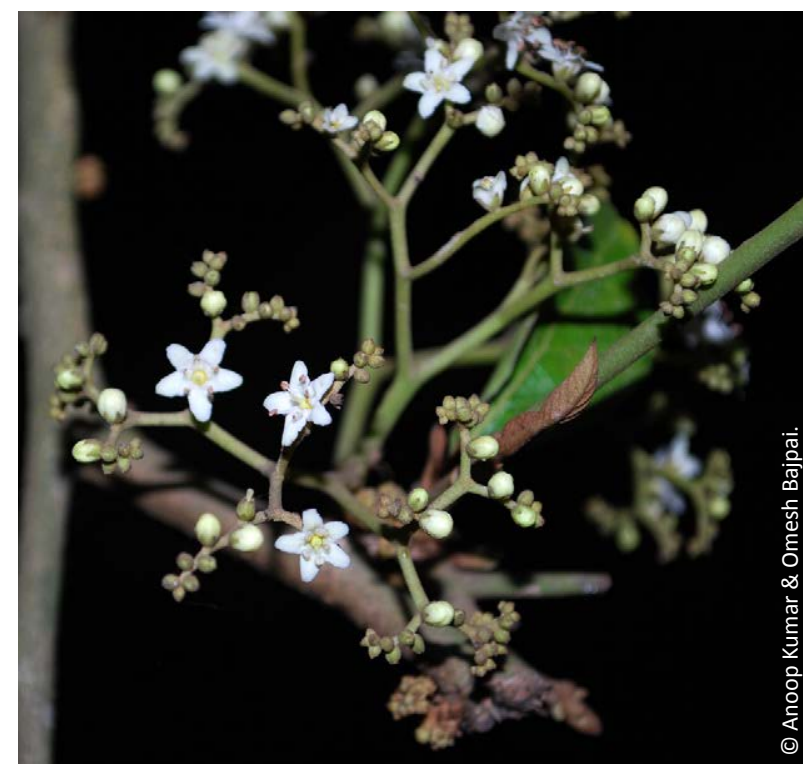

Image 13. Ehretia laevis Roxb.

**Heliotropium strigosum Willd., Sp. PI.1 (2): 743. 1798.

Habit: Perennial herb.

Specimens examined: 26.v.1954, Motipur range, Hira Lal 13435 (LWG).

Trichodesma indicum (L.) Sm., A. Rees Cycl. 36: Trichodesma no. 1. 1817 (R. Br., Prodr. 149. 1810, nom. inval.); Lehm., Pl. Asperif. Nucif. 193. 1818; Duthie, Fl. Upp. Gang. Plain 1: 538. repr. ed. 1960; Panigrahi et al., Bull. Bot. Surv. India 11 (1 \& 2): 108. 1969.

Borago indica L., Sp. PI. 137. 1753. Trichodesma amplexicaule Roth, Nov. Sp. PI. 104. 1821; Clarke in Hook. f., Fl. Brit. India 4: 153. 1883. T. sedgewickianum Banerjee, Bull. Bot. Soc. Beng. 16: 10. 1962; Maliya \& Datt, J. Econ. Taxon. Bot. 34 (1): 57. 2010. T. indicum (L.) Sm. var. amplexicaule (Roth) Cooke, Fl. Bombay 2: 281. 1967; Maliya \& Datt, J. Econ. Taxon. Bot. 34 (1): 57. 2010.

Habit: Annual herb.

Specimens examined:26.viii.2002, Katerniaghat range, coll. S.D. Maliya 223763 (LWG); 26.viii.2002, Katerniaghat range, coll. S.D. Maliya 223774 (LWG); 09.xi.2002, road side, Katerniaghat range, coll. S.D. Maliya 224550 (LWG); 12.xi.2002, Nishangara range, coll. S.D. Maliya 224651 (LWG); 17.iv.2003, road side, Katerniaghat range, coll. S.D. Maliya 224916 (LWG); 22.iv.2003, Nishangara range, coll. S.D. Maliya 224972 (LWG); 05.xi.2008, Katerniaghat range, coll. S.D. Maliya 226645 (LWG).

\section{BRASSICACEAE}

**Brassica rapa L. subsp. campestris (L.) Clapham in Clapham et al., Fl. Brit. Isles 153. 1952; Hajra et al. in Sharma et al., Fl. India 2: 138. 1993; Maliya \& Datt, J. Econ. Taxon. Bot. 34 (1): 46. 2010.

B. campestris L., Sp. PI. 666. 1753.

Habit: Annual herb, cultivated.

Specimens examined: 21.xii.2003, Katerniaghat range, coll. S.D. Maliya 225231 (LWG).

*Brassica rapa L. subsp. trilocularis (Roxb.) Hanelt. in R. Mansfeld, Verz. Landwirtsch. u. Gartn. Kulturpfl., Auf. 2, ed. J. Schultze-Motel, 1: 302. 1986.

Sinapis trilocularis Roxb., Fl. Ind. 3: 121. 1832. Brassica campestris L. var. sarson Prain, Dept. Land Res. \& Agri. Bengal Bull. No. 4: 24, t. 5. 1899; Saini, J. Econ. Taxon. Bot. 29 (3): 537. 2005.

Habit: Herb, cultivated.

Specimens examined: Not observed. Record based on Saini (2005).

**Capsella bursa-pastoris (L.) Medik., Pfl.-Gatt. 1: 85. 1792; Hook. f. \& Anderson in Hook. f., Fl. Brit. India 1: 159. 1872; Bhaumik in Sharma et al., Fl. India 2: 189. 1993; Saini, J. Econ. Taxon. Bot. 29 (3): 537. 2005.

Thlaspi bursa-pastoris L., Sp. PI. 647. 1753.

Habit: Herb.

Specimens examined: Not observed. Record based on Saini (2005).

**Eruca vesicaria (L.) Cav. subsp. sativa (Mill.) Thell., G. Hegi, III. Fl. Mitt.-Eur. 4 (1): 201. 1918.

E. sativa Mill., Gard. Dict. Ed. 8: 1. 1768; Hook. f. \& Anderson in Hook. f., Fl. Brit. India 1: 158. 1872; Hajra et al. in Sharma et al., Fl. India 2: 143. 1993; Maliya \& Datt, J. Econ. Taxon. Bot. 34 (1): 47. 2010.

Habit: Herb, cultivated.

Specimens examined: 21.xii.2003, Katerniaghat range, coll. S.D. Maliya 225233 (LWG).

**Raphanus sativus L., Sp. Pl. 669. 1753; Hook. f. \& Anderson in Hook. f., Fl. Brit. India 1: 166. 1872; Duthie, Fl. Upp. Gang. Plain 1: 47. repr. ed. 1960; Hajra et al. in Sharma et al., Fl. India 2: 145. 1993; Maliya \& Datt, J. Econ. Taxon. Bot. 34 (1): 47. 2010.

Habit: Herb, cultivated.

Specimens examined: 20.iii.2004, Girijapuri forest, coll. S.D. Maliya 225923 (LWG). 


\section{BURSERACEAE}

Garuga pinnata Roxb., Hort. Beng. 33. 1814. \& PI. Coromandel 3: 5, t. 208. 1819; Bennett in Hook. f., Fl. Brit. India 1: 528. 1875; Chitra \& Henry et al. in Hajra et al., FI. India 4: 448. 1997; Maliya \& Datt, J. Econ. Taxon. Bot. 34 (1): 49. 2010.

Habit: Tree.

Specimens examined: 13.iv.1967, Katerniaghat range, S. L. Kapoor \& party 70033 (LWG); 22.xii.2005, Nishangara range, coll. S.D. Maliya 227178 (LWG).

\section{CACTACEAE}

**Hylocereus trigonus (Haw.) Saff., Rep. (Annual) Board Regents Smithsonian Inst. 1908:553. 1909.

Cereus trigonus Haw., Syn. PI. Succ. 3: 181. 1812.

Habit: Herb.

Specimens examined: 25.x.2010, Fakirpuri, near Rampurwa village, coll. S.D. Maliya 252636 (LWG).

* Opuntia cochenillifera (L.) Mill., Gard. dict. ed. 8: Opuntia no. 6. 1768.

Cactus cochenillifera L., Sp. PI. 468. 1753. Nopalea cochenillifera (L.) Solm-Dycl, Cact. Horkl. Dyck. 2: 64. 1850; Saini, J. Econ. Taxon. Bot. 29 (3): 586. 2005.

Habit: Succulent shrub, cultivated.

Specimens examined: Not observed. Record based on Saini (2005)

**Opuntia elatior Mill., Gard. dict. ed. 8: Opuntia no. 4. 1768; Maliya \& Datt, J. Econ. Taxon. Bot. 34 (1): 54. 2010.

Habit: Shrub.

Specimens examined: 17.v.1990, Faquirpur, Motipur range, K.K. Khanna 80 (BSA); 19.vii.2004, Bardia village, coll. S.D. Maliya 225982 (LWG).

\section{CAMPANULACEAE}

Lobelia alsinoides Lam., Encycl. 3: 588. 1792; Panigrahi et al., Bull. Bot. Surv. India 11 (1 \& 2): 105. 1969.

Habit: Succulent annual herb.

Specimens examined: 24.iv.1964, Nishangara range, G. Panigrahi \& O.P. Mishra 6596 (BSA).

Wahlenbergia marginata (Thunb.) A. DC., Monogr. Campan. 143. 1830; Panigrahi et al., Bull. Bot. Surv. India 11 (1 \& 2): 105.1969.

Campanula marginata Thunb. in Murray, Syst. Veg. ed. 14: 211. 1784. C. gracilis Frost., Prodr. 84. 1786. Wahlenbergia gracilis A. DC., Monogr. Campan. 142. 1830; Clarke in Hook. f., Fl. Brit. India 3: 429. 1881.

Habit: Annual herb.
Specimens examined: 12.iii.1964, Murtiha range, G. Panigrahi \& O.P. Mishra 2852 (BSA).

\section{CANNABACEAE}

Cannabis sativa L., Sp. Pl. 1027. 1753; Hook. f. in Hook. f., Fl. Brit. India 5: 487. 1888; Maliya \& Datt, J. Econ. Taxon. Bot. 34 (1): 63. 2010.

Habit: Herb.

Specimens examined: 16.v.1987, K.K. Singh \& team 5968 (LWG); 23.viii.2002, Nishangara range, coll. S.D. Maliya 223635 (LWG); 26.viii.2002, coll. S.D. Maliya 223782 (LWG); 13.xi.2002, Katerniaghat range, coll. S.D. Maliya 224680 (LWG) (Image 14).

**Celtis australis L., Sp. PI. 1043. 1753; Duthie, Fl. Upp. Gang. Plain 3: 218. repr. ed. 1960.

Rhamnus triquetra sensu Maliya \& Datt, J. Econ. Taxon. Bot. 34 (1): 49. 2010.

Habit: Tree.

Specimens examined: 13.iv.1967, S. L. Kapoor 70037 (LWG); 18.iv.2003, Bichhiya, Katerniaghat range, coll. S.D. Maliya 224927 (LWG).

Celtis tetrandra Roxb., Fl. Ind. 2: 63. 1832; Panigrahi et al., Bull. Bot. Surv. India 11 (1 \& 2): 96. 1969.

Habit: Tree.

Specimens examined: 12.ii.1965, Nishangara range,

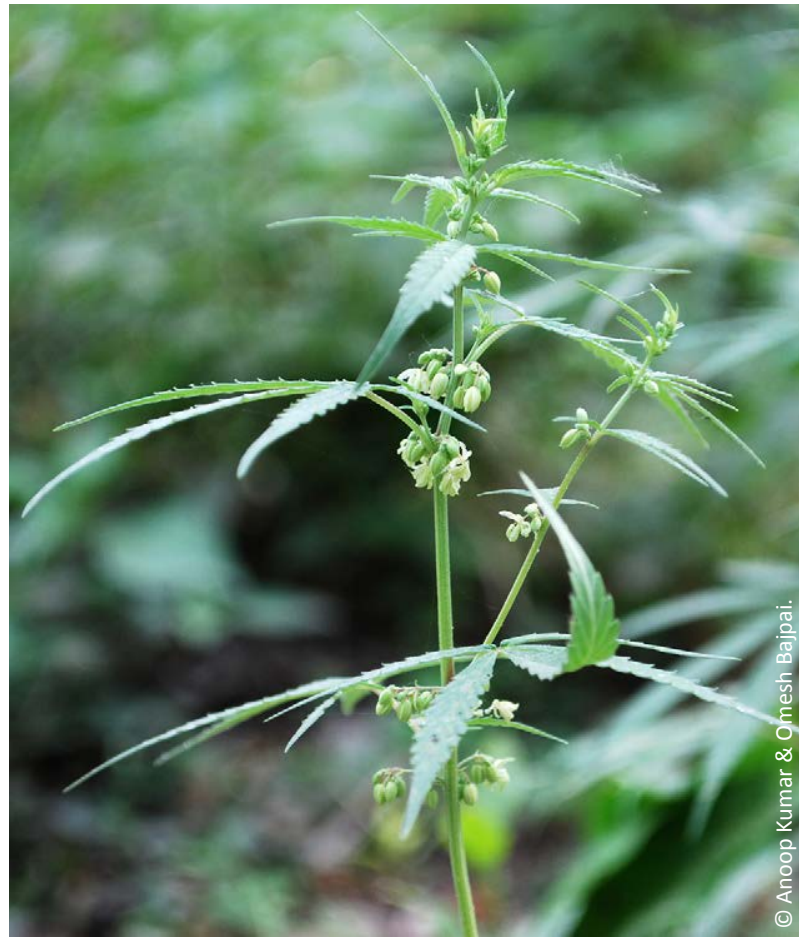

Image 14. Cannabis sativa L. 
O.P. Mishra 7920 (BSA); 05.iii.2011, Behind custom office building, Katerniaghat range, L.B. Chaudhary, A. Kumar \& O. Bajpai, 252861.

Celtis timorensis Spam., Linnaea 15: 343. 1841.

Habit: Tree.

Specimens examined: 09.xii.1986, K.K. Khanna \& R. Saran 37660 (BSA).

\section{CAPPARACEAE}

Capparis zeylanica L., Sp. PI. 720. 1762; Panigrahi et al., Bull. Bot. Surv. India 11 (1 \& 2): 97. 1969; Raghavan in Sharma et al., FI. India 2: 298. 1993; Maliya \& Datt, J. Econ. Taxon. Bot. 34 (1): 47. 2010.

C. horrida L. f., Supp. 264. 1781; Hook. f. \& Thoms. in Hook. f., Fl. Brit. India1: 178. 1872.

Habit: Climbing shrub.

Specimens examined: 25.v.1954, Motipur range, Hira Lal 13409 (LWG); 19.iv.2003, road side, Murtiha range, coll. S.D. Maliya \& B. Datt 224941 (LWG); 22.iv.2003, Nishangara range, coll. S.D. Maliya \& B. Datt 224996 (LWG); 28.viii.2003, Murtiha range, coll. S.D. Maliya 214852 (LWG); 07.x.2009, Kakraha range, coll. S.D. Maliya 226651 (LWG) (Image 15).

\section{CARICACEAE}

*Carica papaya L., Sp. PI. 1036. 1753; Maliya \& Datt, J. Econ. Taxon. Bot. 34 (1): 53. 2010.

Habit: Tree like herb, cultivated.

Specimens examined: Not observed. Record based on Maliya \& Datt (2010).

\section{CARYOPHYLLACEAE}

**Polycarpon prostratum (Forssk.) A. \& S., Osterr. Bot. Z. 39: 128. 1889; Majumdar in Sharma et al., FI. India 2: 553. 1993; Saini, J. Econ. Taxon. Bot. 29 (3): 541. 2005. Alsine prostrata Forssk., Fl. Aegypt-Arab. 207. 1775. Habit: Prostrate herb.

Specimens examined: Not observed. Record based on Saini (2005).

**Spergula arvensis L., Sp. PI. 440. 1753; Edgeworth \& Hook. f. in Hook. f., Fl. Brit. India1: 243. 1874; Duthie, FI. Upp. Gang. Plain 1: 63. repr. ed. 1960; Majumdar in Sharma et al., FI. India 2: 578. 1993; Saini, J. Econ. Taxon. Bot. 29 (3): 541. 2005.

Spergularia arvensis (L.) Cambess, Fl. Bras. Merid. (quarto ed.) 2: 180. 1829.

Habit: Annual herb.

Specimens examined: Not observed. Record based on Saini (2005).

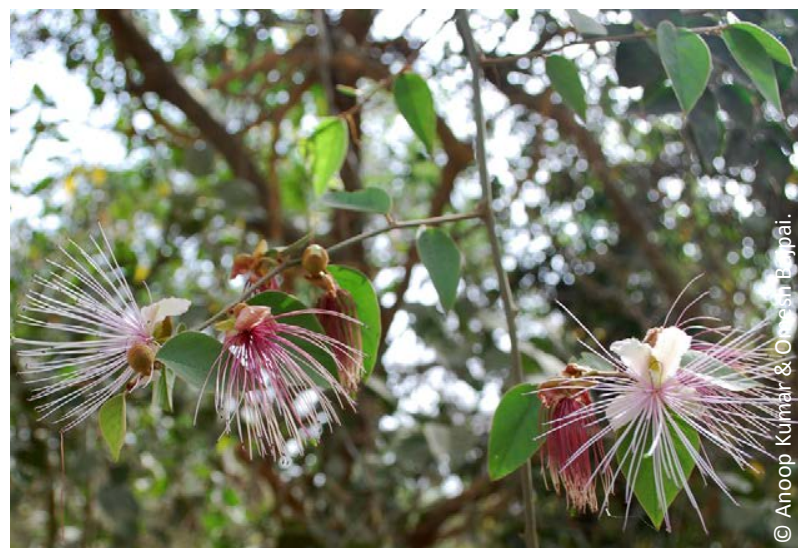

Image 15. Capparis zeylanica L.

**Stellaria media (L.) Vill., Hist. PI. Dauphine. 3(1): 615. 1789; Majumdar in Sharma et al., FI. India 2: 585. 1993; Saini, J. Econ. Taxon. Bot. 29 (3): 541. 2005.

Alsine media L., Sp. PI. 272. 1753.

Habit: Annual herb.

Specimens examined: Not observed. Record based on Saini (2005).

\section{CASUARINACEAE}

*Casuarina equisetifolia L., Amoen. Acad. 4:143. 1759 "equisefolia"; Maliya \& Datt, J. Econ. Taxon. Bot. 34 (1): 64.2010

Habit: Tree, cultivated.

Specimens examined: Not observed. Record based on Maliya \& Datt (2010).

\section{Celastraceae}

Celastrus paniculatus Willd., Sp. PI. 1 (2): 1125. 1798; Lawson in Hook. f., Fl. Brit. India 1: 617. 1875; Saini, J. Econ. Taxon. Bot. 29 (3): 553. 2005; Maliya \& Datt, J. Econ. Taxon. Bot. 34 (1): 49.2010.

Habit: Climber.

Specimens examined: 27.iv.2010, near Bichhiya railway track, pole no. 156/17, A. Kumar \& O. Bajpai 252298 (LWG) (Image 16).

Hippocratea arborea Roxb., Pl. Coromandel 3: 3, pl. 205. 1819; Duthie, Fl. Upp. Gang. Plain 1: 150. repr. ed. 1960.

Habit: Climber.

Specimens examined: 31.x.2009, near Lakkad Shah Baba majr, beat 20, Murtiha range, A. Kumar \& O. Bajpai 252166 (LWG) (Image 17). 


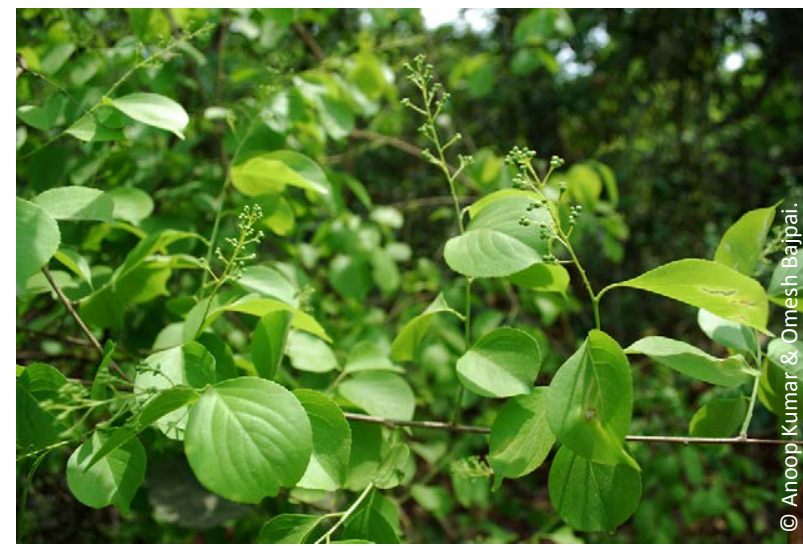

Image 16. Celastrus paniculatus Willd.

\section{Ceratophyllaceae}

Ceratophyllum demersum L., Sp. PI. 992. 1753; Hook. f. in Hook. f. Fl. Brit. India 5: 639. 1888; Duthie, Fl. Upp. Gang. Plain 3: 255. repr. ed. 1960; Panigrahi et al., Bull. Bot. Surv. India 11 (1 \& 2): 102. 1969; Maliya \& Datt, J. Econ. Taxon. Bot. 34 (1): 64. 2010.

Habit: Submerged aquatic herb.

Specimens examined: 12.iii.1964, Motipur range, G. Panigrahi 2851 (BSA); 22.xi.1964, Orla, Motipur range, G. Panigrahi \& O.P. Mishra 6570 (BSA); 24.x.2004, road side, Kailashpuri forest, coll. S.D. Maliya 227103 (LWG).

\section{CHENOPODIACEAE}

Chenopodium album L., Sp. Pl. 219. 1753; Hook. f. in Hook. f., Fl. Brit. India 5: 3. 1886; Duthie, Fl. Upp. Gang. Plain 3: 143. repr. ed. 1960; Panigrahi et al., Bull. Bot. Surv. India 11 (1 \& 2): 103. 1969; Maliya \& Datt, J. Econ. Taxon. Bot. 34 (1): 61.2010.

Habit: Erect herb.

Specimens examined: 12.iii.1964, Murtiha gate, Katerniaghat Wildlife Sanctuary, Bharaich, G. Panigrahi 2866 (BSA); 21.xii.2003, Katerniaghat range, coll. S.D. Maliya 225234 (LWG); 27.xii.2005, Lahaura village, Nishangara village, coll. S.D. Maliya 227190 (LWG).

Chenopodium murale L., Sp. PI. 219. 1753; Hook. f. in Hook. f., Fl. Brit. India 5: 4. 1886; Duthie, Fl. Upp. Gang. Plain 3: 144. repr. ed. 1960; Panigrahi et al., Bull. Bot. Surv. India 11 (1 \& 2): 103. 1969.

Habit: Herb.

Specimens examined: Not observed. Record based on Panigrahi et al. (1969).

**Dysphania ambrosioides (L.) Mosyakin \& Clemants, Ukrayins'k. Bot. Zhurn. new ser., 59: 382. 2002.

Chenopodium ambrosioides L., Sp. PI. 219. 1753; Hook.

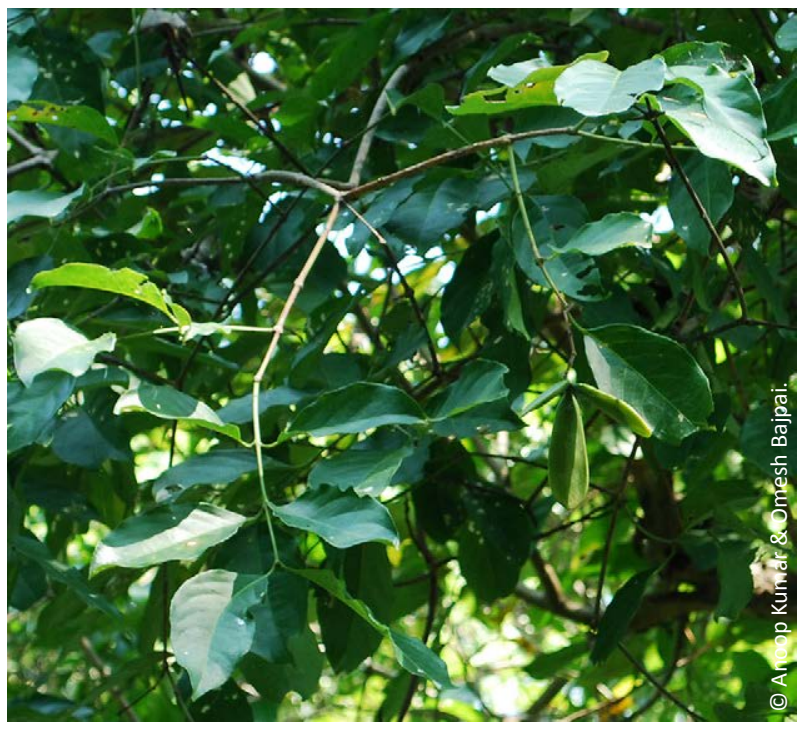

Image 17. Hippocratea arborea Roxb.

f. in Hook. f., Fl. Brit. India 5: 4. 1886; Raizada, Suppl. Fl. Upp. Gang. Plain 336. 1976; Maliya \& Datt, J. Econ. Taxon. Bot. 34 (1): 61.2010.

Habit: Herb.

Specimens examined: 16.vii.2004, Kuthiyaghat forest, coll. S.D. Maliya 225954 (LWG).

\section{Cleomaceae}

Cleome viscosa L., Sp. PI. 672. 1753; Hook. f. \& Thoms. in Hook. f., Fl. Brit. India 1: 170. 1872; Duthie, Fl. Upp. Gang. Plain 1: 48. repr. ed. 1960; Raghavan in Sharma et al., FI. India 2: 317. 1993; Saini, J. Econ. Taxon. Bot. 29 (3): 539. 2005; Maliya \& Datt, J. Econ. Taxon. Bot. 34 (1): 47. 2010.

Arivela viscosa (L.) Raf., Sylva tellur. 110. 1838.

Habit: Annual herb.

Specimens examined: 23.viii.2002, Nishangara range, coll. S.D. Maliya 22654 (LWG); 25.viii.2002, Murtiha range, coll. S.D. Maliya 223727 (LWG); 10.xi.2002, Murtiha range, coll. S.D. Maliya 224586 (LWG).

**Cleome gynandra L., Sp. PI. 671. 1753; Panigrahi et al., Bull. Bot. Surv. India 11 (1 \& 2): 97. 1969; Raghavan in Sharma et al., Fl. India 2: 309. 1993.

Gynandropsis gynandra (L.) Briq., Annuaire Conserv. Jard. Bot. Geneve 17:382. 1914.

Habit: Herb.

Specimens examined: 24.xi.1964, Plantation area, G. Panigrahi \& O.P. Mishra 6610A (BSA). 


\section{COMBRETACEAE}

Anogeissus acuminata (Roxb. ex DC.) Wall. ex Guill. \& Perr. in A. Guillemin, Fl. Seneg. Tent. 1: 280. 1832.

Conocarpus acuminatus Roxb. ex DC., Prodr. 3: 17. 1828 "acuminata". Anogeissus pendula Edgew., (Catal. PI. Banda 47. 1851) J. Asiat. Soc. Bengal 21: 171. 1853; Clarke in Hook. f., Fl. Brit. India 2: 451. 1879; Saini, J. Econ. Taxon. Bot. 29 (3): 576. 2005.

Habit: Tree.

Specimens examined: Not observed. Record based on Saini (2005).

Combretum indicum (L.) De Filipps, Useful plants of the Commonwealth of Dominica, West Indies 277. 1998.

Quisqualis indica L., Sp. Pl. 556. 1762; Clarke in Hook. f., Fl. Brit. India 2: 459. 1879; Saini, J. Econ. Taxon. Bot. 29 (3): 577. 2005

Habit: Scandent shrub.

Specimens examined: Not observed. Record based on Saini (2005)

Combretum roxburghii Spreng., Syst. Veg. 2: 331. 1825; Saini, J. Econ. Taxon. Bot. 29 (3): 576. 2005.

C. decandrum Roxb., Pl. Coromandel 1: 43, t. 59. 1796 (non Jacq.1760); Clarke in Hook. f., Fl. Brit. India 2: 452. 1879.

Habit: Large shrub.

Specimens examined: Not observed. Record based on Saini (2005)

Terminalia arjuna (Roxb. ex DC.) Wight \& Arn., Prodr. FI. Ind. Orient. 314. 1834; Clarke in Hook. f., Fl. Brit. India 2: 447.1879.

Pentaptera arjuna Roxb. ex DC., Prodr. 3: 14. 1828. P. glabra Roxb., FI. Ind. 2: 440. 1832. Terminalia glabra (Roxb.) Wight \& Arn., Prodr. Fl. Ind. Orient. 314. 1834.

Habit: Tree, plantation.

Specimens examined: 25.iv.2010, compartment-8, Goitoiha plantation, Kakraha range, L.B. Chaudhary, A. Kumar \& O. Bajpai 252278 (LWG).

Terminalia bellirica (Gaertn.) Roxb., Pl. Coromande 2: 54, t. 198. 1805; 'bellerica' Clarke in Hook. f., Fl. Brit. India 2: 445. 1879.

Myrobalanus bellirica Gaertn., Fruct. Sem. PI. 2: 90. t. 97. 1790 .

Habit: Tree.

Specimens examined: 25.iv.2010, Goitoiha plantation, Kakraha range, L.B. Chaudhary, A. Kumar \& O. Bajpai 252277 (LWG); 28.iv.2010 Piller no. 76, Indo Nepal Border, L.B. Chaudhary, A. Kumar \& O. Bajpai 252300
(LWG) (Image 18).

Terminalia elliptica Willd., Sp. PI. 4 (2): 969. 1806; Maliya \& Datt, J. Econ. Taxon. Bot. 34 (1): 52. 2010.

T. alata Heyne ex Roth., Nov. PI. Sp. 379. 1821; Kanai, Fl. East Himalaya, 220. 1966; Saini, J. Econ. Taxon. Bot. 29 (3): 577. 2005. Pentaptera tomentosa Roxb., Fl. Ind. 2: 440. 1832. Terminalia tomentosa (Roxb.) Wight \& Arn., Prodr. Fl. India Orient. 314. 1834; Clarke in Hook. f., Fl. Brit. India 2: 447. 1879.

Habit: Tree.

Specimens examined: 27.viii.2002, Murtiha range, coll. S.D. Maliya 223793 (LWG); 20.vii.2004, road side, near bus stand, Nishangara range, coll. S.D. Maliya 225969 (LWG); 26.x.2004, Murtiha range, coll. S.D. Maliya 227123 (LWG).

\section{CONVOLVULACEAE}

Convolvulus arvensis L., Sp. Pl. 153. 1753; Clarke in Hook. f., Fl. Brit. India 4: 219. 1883; Duthie, Fl. Upp. Gang. Plain 1: 548. repr. ed. 1960; Saini, J. Econ. Taxon. Bot. 29 (3): 621. 2005; Maliya \& Datt, J. Econ. Taxon. Bot. 34 (1): 57. 2010.

Habit: Herb with creeping root stock.

Specimens examined: 10.xii.1986, Motipur range, K.K. Khanna \& R. Saran 37699 (BSA); 20.iii.2004, Girijapuri forest, coll. S.D. Maliya 225928 (LWG).

Convolvulus prostratus Forssk., Fl. Aegypt.-Arab. 203. 1775; Maliya, J. Econ. Taxon. Bot. 36 (2): 422. 2012.

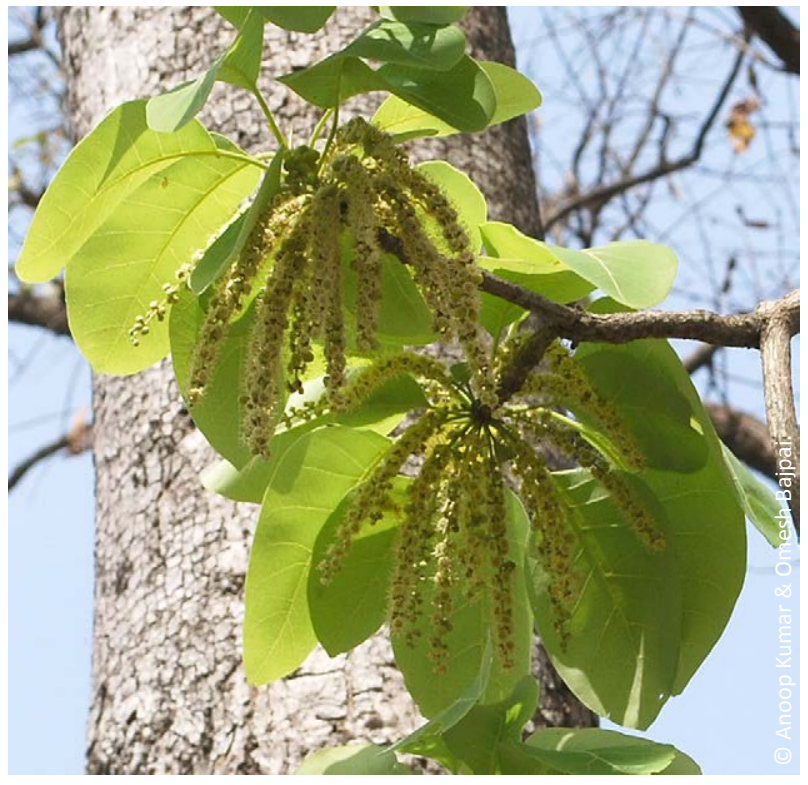

Image 18. Terminalia bellirica (Gaertn.) Roxb. 
Habit: Herb.

Specimens examined: Not observed. Record based on Maliya \& Datt (2012).

Cuscuta reflexa Roxb., PI. Coromandel 2: 3, t. 104. 1798; Clarke in Hook. f., Fl. Brit. India 4: 225. 1883; Duthie, Fl. Upp. Gang. Plain 1: 543. repr. ed. 1960; Panigrahi et al., Bull. Bot. Surv. India 11 (1 \& 2): 108. 1969; Maliya \& Datt, J. Econ. Taxon. Bot. 34 (1): 58. 2010.

C. macrantha G. Don, Gen. Syst. 4: 305. 1838.

Habit: Twining parasitic herb.

Specimens examined: 21.v.1987, K.K. Singh \& party 5979 (LWG); 31.viii.2003, Amba forest, coll. S.D. Maliya 214891 (LWG); 24.xii.2003, Kuthiyaghat forest, coll. S.D. Maliya 225255 (LWG); 10.x.2009, near Bichhiya town, coll. S.D. Maliya 226676 (LWG).

Erycibe wightiana Graham., Cat. PI. Bomb. 137. 1839; Panigrahi et al., Bull. Bot. Surv. India 11 (1 \& 2): 106.1969. Habit: Climber.

Specimens examined: 12.iii.1964, Murtiha gate, Murtiha range, G. Panigrahi 2877 (BSA) (Image 19).

Evolvulus alsinoides (L.) L., Sp. PI. 392. 1762; Clarke in Hook. f., Fl. Brit. India 4: 220. 1883; Duthie, Fl. Upp. Gang. Plain 1: 526. repr. ed. 1960; Maliya \& Datt, J. Econ. Taxon. Bot. 34 (1): 57. 2010.

Convolvulus alsinoides L., Sp. PI. 157. 1753.

Habit: Annual herb.

Specimens examined: 16.iii.2004, Karmohini forest, coll. S.D. Maliya 225290 (LWG).

Evolvulus nummularius (L.) L., Sp. PI. 391. 1762.

Convolvulus nummularis L., Sp. PI. 157. 1753. Volvulopsis nummularium (L.) Robert, Candollea 14: 28. 1952; Raizada, Suppl. Fl. Upp. Gang. Plain 166. 1976; Panigrahi et al., Bull. Bot. Surv. India 11 (1 \& 2): 106. 1969; Maliya \& Datt, J. Econ. Taxon. Bot. 34 (1): 57. 2010.

Habit: Herb.

Specimens examined: 23.viii.2002, Nishangara range, coll. S.D. Maliya 223639 (LWG).

*Ipomoea batatas (L.) Lam., Encycl. 1: 465. 1793, Maliya \& Datt, J. Econ. Taxon. Bot. 34 (1): 58. 2010.

Convolvulus batatas L., Sp. PI. 154. 1753.

Habit: Herb, cultivated.

Specimens examined: 27.xii.2005, Lahaura village, Nishangara range, coll. S.D. Maliya 227196 (LWG).

*Ipomoea cairica (L.) Sweet., Hort. Brit. 2: 287. 1827; Raizada, Suppl. FI. Upp. Gang. Plain 162. 1976; Maliya \&

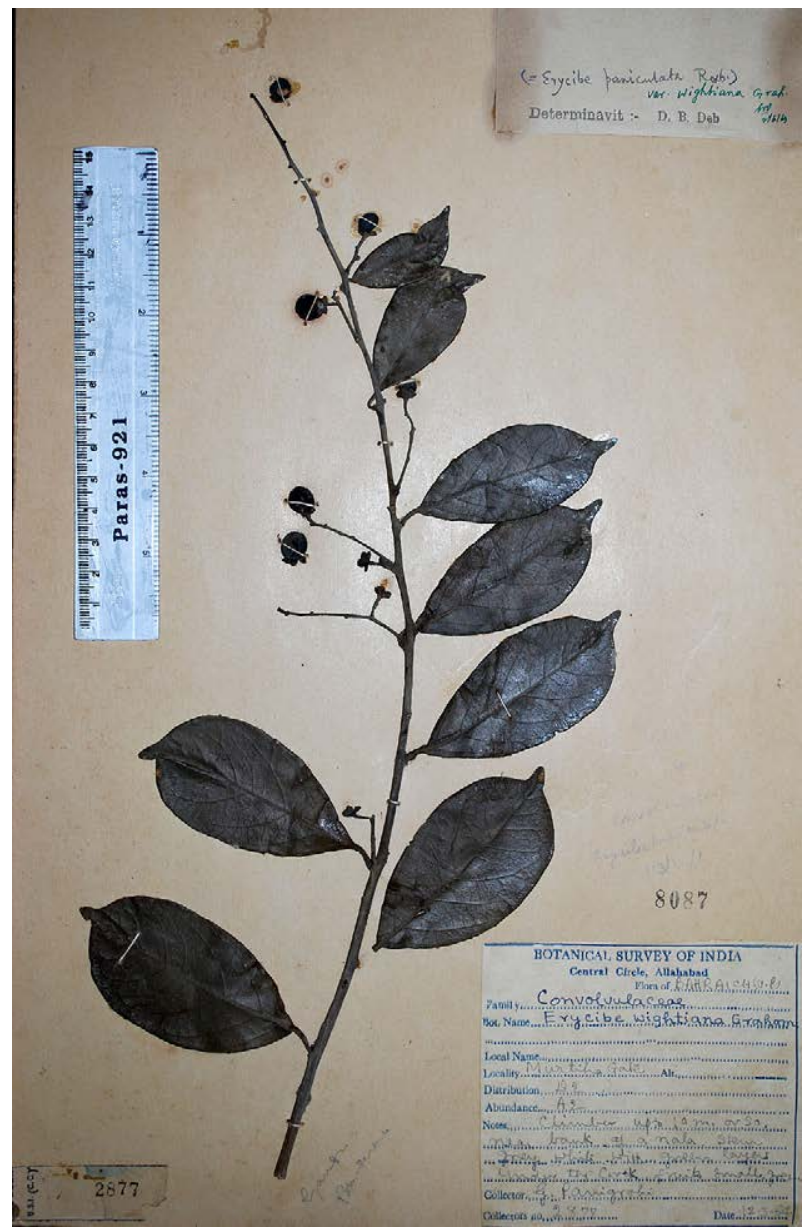

Image 19. Erycibe wightiana Graham.

Datt, J. Econ. Taxon. Bot. 34 (1): 58. 2010.

Convolvulus caricus L., Syst. Nat. 2: 922. 1759. Ipomoea palmata Forssk., Fl. Aegypt.- Arab. 43. 1775; Clarke in Hook. f., FI. Brit. India 4: 214. 1883.

Habit: Climbing shrub, cultivated, ornamental.

Specimens examined: 26.viii.2002, Katerniaghat range, coll. S.D. Maliya 223770 (LWG); 09.xi.2002, rest house, Katerniaghat range, coll. S.D. Maliya 224526 (LWG); 27.x.2004, Katerniaghat range, coll. S.D. Maliya 227128 (LWG).

**Ipomoea eriocarpa R. Br., Prodr. 484. 1810.

I. sindica Stapf., Bull. Misc. Inform. Kew 346. 1894; Duthie, Fl. Upp. Gang. Plain 1: 554. repr. ed. 1960; Saini, J. Econ. Taxon. Bot. 29 (3): 624. 2005.

Habit: Twining annual herb.

Specimens examined: Not observed. Record based on Saini (2005).

**Ipomoea fistulosa Mart. ex Choisy., Prodr. 9: 349. 1845; Saini, J. Econ. Taxon. Bot. 29 (3): 622. 2005. 
I. carnea Jacq. ssp. fistulosa (Mart. ex Choisy) Austin, Taxon 26 (2-3): 237. 1977; Maliya \& Datt, J. Econ. Taxon. Bot. 34 (1): 58. 2010.

Habit: Large straggling shrub.

Specimens examined: 27.viii.2003, near Geruwa river, Katerniaghat range, coll. S.D. Maliya 214844 (LWG).

Ipomoea nil (L.) Roth, Catal. Bot. 1: 36. 1797; Saini, J. Econ. Taxon. Bot. 29 (3): 623. 2005.

Convolvulus nil L., Sp. Pl. 219. 1762.

Habit: Twining annual herb.

Specimens examined: Not observed. Record based on Saini (2005)

**Ipomoea pes-tigridis L., Sp. Pl. 162. 1753; Clarke in Hook. f., Fl. Brit. India 4: 204. 1883; Duthie, Fl. Upp. Gang. Plain 1: 557. repr. ed. 1960; Maliya \& Datt, J. Econ. Taxon. Bot. 34 (1): 58. 2010.

Habit: Twining annual herb.

Specimens examined: 12.xi.2002, Nishangara range, coll. S.D. Maliya 224647 (LWG).

**Ipomoea quamoclit L., Sp. PI. 159. 1753; Maliya \& Datt, J. Econ. Taxon. Bot. 34 (1): 58. 2010.

Habit: Scandent climbing herb.

Specimens examined: 09.xi.2002, near Bichhiya town, coll. S.D. Maliya 224505 (LWG); 12.xi.2002, Nishangara range, coll. S.D. Maliya 224629 (LWG).

**Ipomoea reptans Poir., Encycl. 3(2): 460. 1814; Duthie, Fl. Upp. Gang. Plain 1: 555. repr. ed. 1960.

I. aquatica Forssk., Fl. Aegypt.- Arab. 44. 1775; Clarke in Hook. f., Fl. Brit. India 4: 210. 1883; Panigrahi et al., Bull. Bot. Surv. India 11 (1 \& 2): 106. 1969; Maliya \& Datt, J. Econ. Taxon. Bot. 34 (1): 57. 2010.

Habit: Aquatic herb.

Specimens examined: 24.x.2004, Kailashpuri forest, coll. S.D. Maliya 227102 (LWG); 27.xii.2005, near Lahaura village, Nishangara village, coll. S.D. Maliya 227195 (LWG).

Merremia hederacea (Burm. f.) Hallier f. in Engl. Bot. Jahrb. Syst. 18: 118. 1893; Panigrahi et al., Bull. Bot. Surv. India 11 (1 \& 2): 106. 1969.

Evolvulus hederaceus Burm. f., Fl. Ind.77. t. 30, f. 2. 1768. Ipompea chryseides Ker Gawl., Bot. Reg. 4: t. 270. 1818. Merremia chryseides (Ker Gawl.) Hallier f. in Engl., Bot. Jahrb. 16: 552. 1893; Duthie, Fl. Upp. Gang. Plain 1: 550. repr. ed. 1960.

Habit: Herb.

Specimens examined: 07.xii.1986, Motipur range, K.K. Khanna \& R. Saran 38571 (BSA); 07.x.2009, Kakraha range, coll. S.D. Maliya 226654 (LWG).

Merremia umbellata (L.) Hallier f., Bot. Jahrb. Syst. 16: 552. 1893; Panigrahi et al., Bull. Bot. Surv. India 11 (1 \& 2): 106.1969.

Convolvulus umbellatus L., Sp. PI. 155. 1753.

Habit: Climber.

Specimens examined: 22.xi.1964, Motipur range, G. Panigrahi \& O.P. Mishra 6545 (BSA) (Image 20).

Operculina turpethum (L.) S. Manso, Enum. subst. braz. 16. 1836 "turpetum"; Panigrahi et al., Bull. Bot. Surv. India 11 (1 \& 2): 106. 1969; Maliya \& Datt, J. Econ. Taxon. Bot. 34 (1): 58. 2010.

Convolvulus turpethum L., Sp. PI. 155. 1753. Ipomoea turpethum (L.) R. Br., Prodr. 485. 1810.

Habit: Climbing perennial herb.

Specimens examined: 09.xi.2002, near Bichhiya town, coll. S.D. Maliya 224503 (LWG); 05.xi.2008, coll. S.D. Maliya 226633 (LWG); 10.x.2009, near Bichhiya town, Katerniaghat range, coll. S.D. Maliya 226675 (LWG).

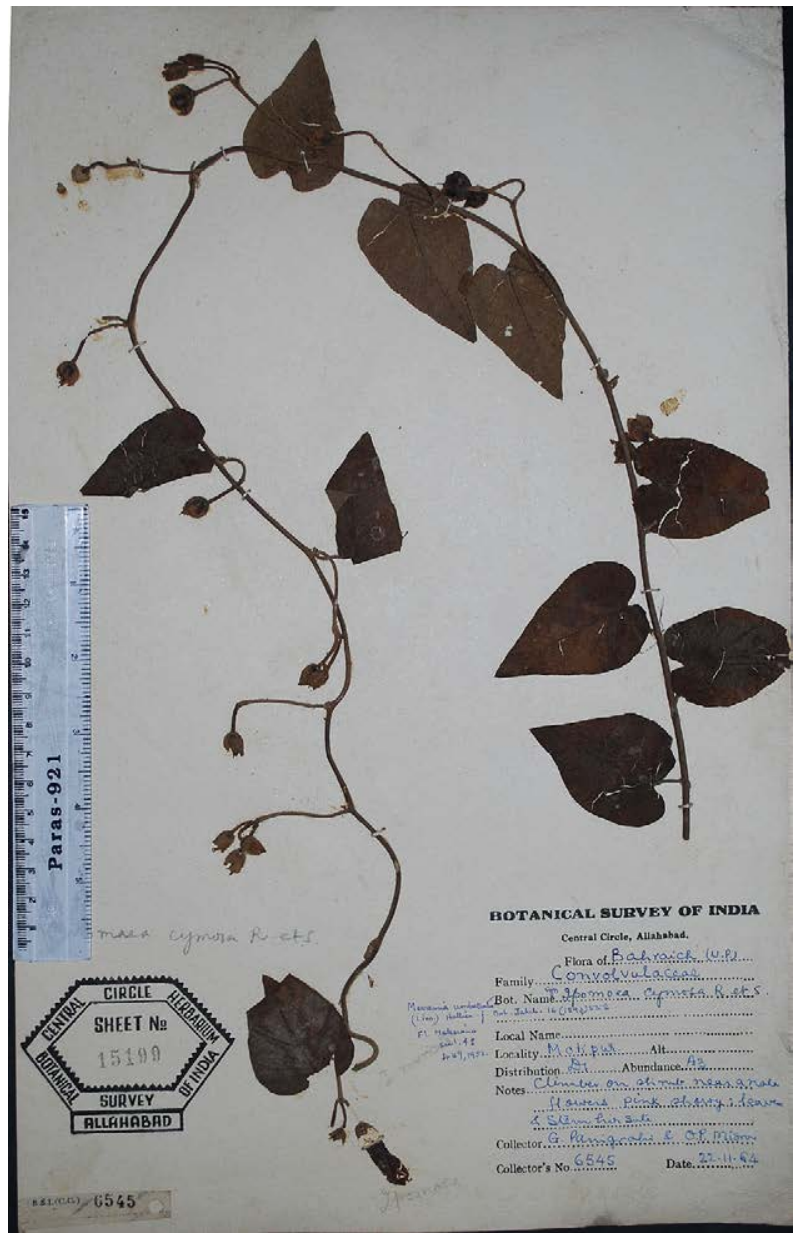

Image 20. Merremia umbellata (L.) Hallier f. 
Porana paniculata Roxb., PI. Coromandel 3: 31, t. 235. 1819; Clarke in Hook. f., Fl. Brit. India 4: 222. 1883; Duthie, Fl. Upp. Gang. Plain 1: 544. repr. ed. 1960; Maliya \& Datt, J. Econ. Taxon. Bot. 34 (1): 58. 2010.

Habit: Large climbing shrub.

Specimens examined: 09.xi.2002, near Bichhiya town railway track, coll. S.D. Maliya 224515 (LWG).

\section{CORNACEAE}

Alangium salviifolium (L. f.) Wang. in Engler, Pflanzenr. IV. 220 B(Heft 41): 9. 1910; Panigrahi et al., Bull. Bot. Surv. India 11 (1 \& 2): 96. 1969.

Grewia salviifolia L. f., Suppl. PI. 409. 1781. Alangium salviifolium subsp. decapetalum (Lam.) Wangerin., Pflanzenr. IV. 220b: 11. 1910; Saini, J. Econ. Taxon. Bot. 29(3): 589. 2005. A. decapetalum Lam., Encycl. 1(1): 174. 1783.

Habit: Shrub or small tree.

Specimens examined: 26.v.1954, Motipur forest, Hira Lal 13463 (LWG); 13.iii.1964, Motipur range, G. Panigrahi 2533 (BSA) (Image 21).

\section{Crassulaceae}

*Kalanchoe integra (Medik.) Kuntze, Rev. Gen. PI. 1: 229. 1891; Becker, Fl. Malesiana 4: 202. 1951; Maliya \& Datt, J. Econ. Taxon. Bot. 34 (1): 52. 2010.

Cotyledon integra Medic., Acta Acad. Theod. Palat. 3: 200, t. 9. 1775. Kalanchoe spathulata DC., Hist. PI. Grasses, t. 65. 1799; Clarke in Hook. f., Fl. Brit. India 2: 414. 1879.

Habit: Succulent perennial herb, cultivated.

Specimens examined: 24.iv.2003, Murtiha chawani, Murtiha range, coll. S.D. Maliya 214834 (LWG).

\section{CUCURBITACEAE}

Actinostemma tenerum Griff., Account Bot. Coll. Cantor 25, PI. 3. 1837; Duthie, Fl. Upp. Gang. Plain 1: 351. repr. ed. 1960; Panigrahi et al., Bull. Bot. Surv. India 11 (1 \& 2): 97.1969.

Habit: Climbing scandent herb.

Specimens examined: 22.xi.1964, Motipur range, G. Panigrahi \& O.P. Mishra 6542 (BSA).

Benincasa hispida (Thunb.) Cogn. in Murray, Syst. Veg. ed. 14: 868. 1784; DC., Mongr. Phan. 3: 513. 1881; Duthie, Fl. Upp. Gang. Plain 1: 338. repr. ed. 1960; Maliya \& Datt, J. Econ. Taxon. Bot. 34 (1): 53. 2010.

Cucurbita hispida Thunb., Fl. Jap. 322. 1784. Benincasa cerifera Savi, Bibl. Ital. 9: 158. 1818; Clarke in Hook. f., Fl. Brit. India 2: 616. 1879.

Habit: Annual climber.

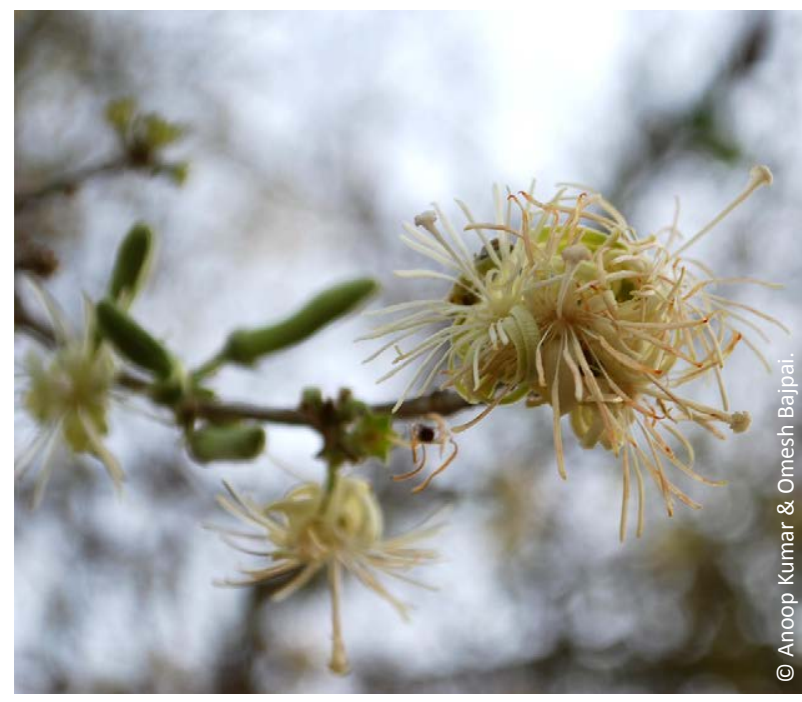

Image 21. Alangium salviifolium (L. f.) Wang.

Specimens examined: 28.xii.2005, Kuthiyaghat, coll. S.D. Maliya 226603 (LWG); 30.x.2009, Murtiha range, A. Kumar, O. Bajpai 252159 (LWG).

*Coccinia grandis (L.) Voigt, Hort. Suburb Calc. 59. 1845; Maheshwari, Bull. Bot. Surv. India 5: 128. 1963; Maliya \& Datt, J. Econ. Taxon. Bot. 34 (1): 53. 2010.

Bryonia grandis L., Mant. PI. 1: 126. 1767. Cephalandra indica Naudin, Ann. Sci. Nat. (Ser. 5) 5: 16. 1866; Clarke in Hook. f., Fl. Brit. India 2: 621. 1879; Duthie, Fl. Upp. Gang. Plain 1: 345. repr. ed. 1960. Coccinia cordifolia (L.) Cogn. Monogr. Phan. 3: 529. 1881.

Habit: Scandent herb, cultivated.

Specimens examined: 21.v.1987, K.K. Singh \& party 5973 (LWG); 31.viii.2003, Fakirpur village, coll. S.D. Maliya 214879 (LWG); 27.xii.2005, Lahura village, coll. S.D. Maliya 227188 (LWG); 26.vi.2011, Chawni, Murtiha range, Awadhesh Srivastava 263543 (LWG); 26.vi.2011, NaiBasti, Awadhesh Srivastava 263546 (LWG); 13.ix.2011, Rampurwa, Awadhesh Srivastava 263558 (LWG).

Cucumis melo forma agrestis Wilde \& Duy., Fl. Malesiana ser. 1. 19: 55. 2010.

Cucumis melo sensu Maliya \& Datt, J. Econ. Taxon. Bot. 34 (1): 53. 2010.

Habit: Climbing herb.

Specimens examined: 26.v.1954, Motipur range, Hira Lal 13420 (LWG); 31.viii.2003, Fakirpur village, coll. S.D. Maliya 214880 (LWG); 19.vii.2004, Kutiyaghat forest, coll. S.D. Maliya 225974 (LWG); 26.vi.2011, Nai Basti, Awadhesh Srivastava 263548 (LWG). 
*Cucumis sativus L., Sp. PI. 1012. 1753.

Habit: Annual climbing herb, cultivated.

Specimens examined: 26.vi.2011, Nai Basti, Awadhesh Srivastava 263550 (LWG).

**Diplocyclos palmatus (L.) Jeffrey, Kew Bull. 15 (3): 352. 1962; Maheshwari, Bull. Bot. Surv. India 5 (2): 128. 1963; Panigrahi et al., Bull. Bot. Surv. India 11 (1 \& 2): 97. 1969; Maliya \& Datt, J. Econ. Taxon. Bot. 34 (1): 53. 2010.

Bryonia palmata L., Sp. PI. 1012. 1753. B. laciniosa auct. non (L.) Naudin, Ann. Sci. Nat., Bot., ser. 56. 1866.

Habit: Climbing herb.

Specimens examined: 20.xi.1964, Nishangara range, G. Panigrahi \& O.P. Mishra 6496 (BSA); 21.xi.1964, Nishangara range, G. Panigrahi \& O.P. Mishra 6512 (BSA); 12.xi.2002, Nishangara range, coll. S.D. Maliya 224631 (LWG); 27.xii.2003, Nishangara range, coll. S.D. Maliya 225274 (LWG); 22.x.2004, Kutiyaghat forest, coll. S.D. Maliya 225990 (LWG); 08.x.2009, near railway line, Murtiha range, coll. S.D. Maliya 226660 (LWG); 23.x.2009, rest house, Kakraha range, A. Kumar \& O. Bajpai 250691 (LWG); 15.ix.2011, Nai Basti, Kakraha range, Awadhesh Srivastava 263593 (LWG).

Gymnopetalum chinense (Lour.) Merr., Phillipp. J. Sci.256. 1919; Wilde \& Duy., Blumea 51. 283. 2006.

Evonymus chinensis Lour., Fl. Cochinch. 156. 1790. Bryonia cochinchinensis Lour., Fl. Cochinch. 2: 595596. 1790. Gymnopetalum cochinchinense (Lour.) Kurz, J. Asiat. Soc. Bengal, Pt. 2, Nat. Hist. 40 (2): 57. 1871. Trichosanthes dicaelospermum sensu Maliya \& Datt, J. Econ. Taxon. Bot. 34 (1): 54. 2010.

Habit: Climbing herb.

Specimens examined: 02.ix.2003, Kuthiyaghat forest, coll. S.D. Maliya 225209 (LWG); 08.ix.2005, Kuthiyaghat forest, coll. S.D. Maliya 227161 (LWG).

Luffa acutangula (L.) Roxb., Fl. Ind. 3: 713.1832 (Hort. Beng. 70. 1814); Wilde \& Duy., Fl. Malesiana ser. 1. 19: 101. 2010.

Cucumis acutangulus L., Sp. PI. 1011. 1753.

Habit: Climbing herb.

Specimens examined: 13.ix.2011, Rampurwa, Awadhesh Srivastava 263555 (LWG).

* Luffa aegyptiaca Mill., Gard. Dict. (ed. 8) Luffa. 1768; Clarke in Hook. f., Fl. Brit. India 2: 614. 1879; Wilde \& Duy., FI. Malesiana ser. 1. 19: 103. 2010.

Luffa cylindrica (L.) M. Roem., Fam. Nat. Syn. Monogr. 2: 63. 1846; Maliya \& Datt, J. Econ. Taxon. Bot. 34 (1): 53. 2010. Momordica cylindrica L., Sp. PI. 1009. 1753.
Habit: Climbing herb, cultivated.

Specimens examined: 21.xii.2003, coll. S.D. Maliya 225221 (LWG); 26.vi.2011, Nidhipurwa, Awadhesh Srivastava 263549 (LWG); 26.vi.2011, Nidhipurwa, Awadhesh Srivastava 263552 (LWG); 13.ix.2011, Rampurwa, Awadhesh Srivastava 263559 (LWG); 30.x.2009, Murtiha range, A. Kumar \& O. Bajpai 252160 (LWG).

** Luffa graveolens Roxb., Fl. Ind. 3:716.1832

Habit: Climber.

Specimens examined: 14.xi.2002, Murtiha range, coll. S.D. Maliya 224678 (LWG); 31.xi.2009, Sujauli road, A. Kumar \& O. Bajpai 252168 (LWG).

* Luffa hermaphrodita Singh \& Bhandari, Baileya 11(4): 133. 1963.

Habit: Climber, cultivated.

Specimens examined: 13.ix.2011, Rampurwa, Awadhesh Srivastava 263556 (LWG); 13.ix.2011, Rampurwa, Awadhesh Srivastava 263557 (LWG); 13.ix.2011, Rampurwa, Awadhesh Srivastava 263561 (LWG).

Momordica charantia L., Sp. Pl. 1009. 1753; Clarke in Hook. f., Fl. Brit. India 2: 616. 1879; Duthie, FI. Upp. Gang. Plain 1: 338. repr. ed. 1960; Maliya \& Datt, J. Econ. Taxon. Bot. 34 (1): 53. 2010.

Habit: Climber.

Specimens examined: 24.viii.2002, Nishangara range, coll. S.D. Maliya 223676 (LWG); 26.viii.2002, Katerniaghat range, coll. S.D. Maliya 223775 (LWG); 10.xi.2002, Murtiha range, coll. S.D. Maliya 224581 (LWG); 05.ix.2005, Girijapuri forest, coll. S.D. Maliya 227152 (LWG); 23.x.2009, rest house, Kakraha range, A. Kumar \& O. Bajpai 250692 (LWG).

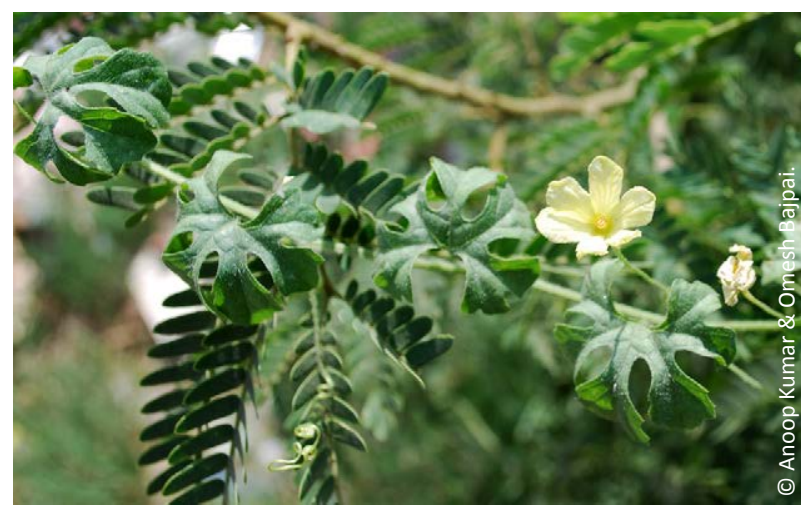

Image 22. Momordica charantia L. forma abbreviata (Ser.) Wild. \& Duy. 
Momordica charantia L. forma abbreviata (Ser.) Wild. \& Duy., Sandakania 17: 73, f. 8. 2008 (2007) \& FI. Malesiana ser. 1. 19: 111. 2010.

Habit: Climber.

Specimens examined: 28.xii.2005, Kutiyaghat forest, coll. S.D. Maliya 226602 (LWG); 27.iv.2010, Murtiha range, A. Kumar \& O. Bajpai 252802 (LWG); 26.iii.2011, rest house, Kakraha range, Awadhesh Srivastava 263506 (LWG); 26.iii.2011, rest house, Kakraha range, Awadhesh Srivastava 263504 (LWG); 14.ix.2011, Kailashpuri forest, Awadhesh Srivastava 263575 (LWG) (Image 22).

Momordica dioica Roxb. ex Willd., Sp. PI. 4 (1): 605. 1805; Clarke in Hook. f., Fl. Brit. India 2: 617. 1879; Duthie, FI. Upp. Gang. Plain 1: 339. repr. ed. 1960; Maliya \& Datt, J. Econ. Taxon. Bot. 34 (1): 53. 2010.

Habit: Climber.

Specimens examined: 28.viii.2003, rest house, Murtiha range, coll. S.D. Maliya 214855 (LWG); 28.viii.2003, rest house, Katerniaghat Wildlife Sanctuary, Murtiha range, Bahraich, coll. S.D. Maliya 214856 (LWG); 29.viii.2003, Nishangara range, coll. S.D. Maliya 214861 (LWG); 30.viii.2003, rest house, Murtiha range, coll. S.D. Maliya 214865 (LWG); 07.ix.2005, Kailashpuri forest, coll. S.D. Maliya 227158 (LWG).

Mukia maderaspatana (L.) M. Roem., Fam. Nat. Syn. Monogr. 2: 47. 1846; Maliya \& Datt, J. Econ. Taxon. Bot. 34 (1): 53. 2010.

Cucumis maderaspatanus L., Sp. PI. 1012. 1753. Melothria maderaspatana (L.) Cogn., DC. Monogr. Phan. 3: 623. 1881; Duthie, Fl. Upp. Gang. Plain 1: 347. repr. ed. 1960.

Bryonia scabrella L., Suppl. PI. 424. 1781.

Habit: Annual scandent herb.

Specimens examined: 04.xi.2008, Murtiha range, Kateniaghat wildlife sanctuary, Bahraich coll. S.D. Maliya 226627 (LWG); 26.x.2004, Murtiha range, Kateniaghat wildlife sanctuary, Bahraich, coll. S.D. Maliya 227119 (LWG).

Solena amplexicaulis (Lam.) Gandhi, Fl. Hassan Dist. 179. 1976: Jeffery, Kew Bull. 34: 793. 1980; Maliya \& Datt, J. Econ. Taxon. Bot. 34 (1): 53. 2010.

Bryonia amplexicaulis Lam., Encycl. 1 (2): 496. 1785 Bryonia umbellata Klein ex Willd., Sp. PI. 4 (1): 618. 1805. Zehneria umbellata (Klein ex Willd.) Thw., Enum. PI. Zeyl. 125. 1859; Clarke in Hook. f., Fl. Brit. India 2: 625. 1879.

Habit: Climber.

Specimens examined: 24.viii.2002, Nishangara range, K.K. Singh \& coll. S.D. Maliya 223674 (LWG).
Trichosanthes cucumerina L., Sp. PI. 1008. 1753; Clarke in Hook. f., Fl. Brit. India 2: 609. 1879; Duthie, Fl. Upp. Gang. Plain 1: 333. repr. ed. 1960; Panigrahi et al., Bull. Bot. Surv. India 11 (1 \& 2): 97. 1969; Maliya \& Datt, J. Econ. Taxon. Bot. 34 (1): 53. 2010.

Habit: Climbing herb.

Specimens examined: 24.viii.2002, Nishangara range, coll. S.D. Maliya 223686 (LWG); 05.ix.2005, Girijapuri forest, coll. S.D. Maliya 227150 (LWG).

*Trichosanthes cucumerina L. var. anguina (L.) Haines, Bot. Bihar Orissa 3: 388. 1922.

Trichosanthes anguina L., Sp. PI. 1008. 1753; Clarke in Hook. f., Fl. Brit. India 2: 610. 1879; Saini, J. Econ. Taxon. Bot. 29 (3): 586. 2005.

Habit: Climbing herb, cultivated, found as an escape.

Specimens examined: Not observed. Record based on Saini (2005).

*Trichosanthes dioica Roxb., Fl. Ind. 3: 701. 1832; Maliya \& Datt, J. Econ. Taxon. Bot. 34 (1): 54. 2010.

Habit: Climbing herb, cultivated.

Specimens examined: 24.iv.2003, Murtiha range, coll. S.D. Maliya 214825 (LWG); 30.x.2009, near Geruwa river, A. Kumar \& O. Bajpai 252157 (LWG); 26.vi.2011, Nai Basti, Awadhesh Srivastava 263544 (LWG); 26.vi.2011, Nai Basti, Awadhesh Srivastava 263547 (LWG); 26.vi.2011, Nai Basti, Awadhesh Srivastava 263551 (LWG); 13.ix.2011, Rampurwa, Awadhesh Srivastava 263562 (LWG); 15.ix.2011, Nai Basti, Awadhesh Srivastava 263595 (LWG); 15.ix.2011, Nai Basti, Awadhesh Srivastava 263596 (LWG).

Trichosanthes tricuspidata Lour., Fl. Cochinch. 2: 589. 1790; Maliya \& Datt, J. Econ. Taxon. Bot. 34 (1): 54. 2010. Modecca bracteata Lam., Encycl. 4: 210. 1797. Trichosanthes palmata Roxb., Fl. Ind. 3: 704. 1832. T. bracteata (Lam.) Voigt., Hort. suburb. Calcutt. 58. 1845.

Habit: Climber.

Specimens examined: 19.vii.2004, Girijapuri forest, coll. S.D. Maliya 225980 (LWG); 09.ix.2005, rest house, Murtiha range, coll. S.D. Maliya 227171 (LWG); 02,ix.2009, near Girijapuri petrol pump, A. Kumar \& O. Bajpai 252178 (LWG).

Zehneria scabra (L. f.) Sond., Fl. Cap. 2: 486. 1862; Maliya \& Datt, J. Econ. Taxon. Bot. 34 (1): 54. 2010.

Bryonia scabra L. f., Suppl. PI. 423. 1871.

Habit: Climber.

Specimens examined: 09.xi.2002, Bichhiya along the railway station, coll. S.D. Maliya 224510 (LWG). 


\section{Dilleniaceae}

Dillenia indica L., Sp. PI. 535. 1753; Hook. f. \& Thoms. in Hook. f., Fl. Brit. India1: 36. 1872; Majumdar in Sharma et al., Fl. India 1: 155. 1993; Saini, J. Econ. Taxon. Bot. 29 (3): 534. 2005.

Habit: Tree.

Specimens examined: Not observed. Record based on Saini (2005).

Dillenia pentagyna Roxb., PI. Coromandel 1: 121, t. 20. 1795; Hook. f. \& Thoms. in Hook. f., Fl. Brit. India 1: 38. 1872; Majumdar in Sharma et al., FI. India 1: 156. 1993; Maliya \& Datt, J. Econ. Taxon. Bot. 34 (1): 46. 2010.

Habit: Tree.

Specimens examined: 27.v.1954, Motipur forest, coll. Hira Lal 13475 (LWG); 29.i.2005, Nishangara forest, coll. S.D. Maliya 224692 (LWG); 09.ix.2005, Murtiha range, coll. S.D. Maliya 227168 (LWG) (Image 23).

\section{DIPTEROCARPACEAE}

Shorea robusta Gaertn. f., Suppl. Carp. 48, t. 186, f. 1. 1805; Dyer in Hook. f., Fl. Brit. India 1: 306. 1874; Janardhanan in Sharma et al., Fl. India 3: 237. 1993; Panigrahi et al., Bull. Bot. Surv. India 11 (1 \& 2): 99. 1969; Maliya \& Datt, J. Econ. Taxon. Bot. 34 (1): 47. 2010.

Habit: Tree.

Specimens examined: 25.v.1954, Motipur range, Hira Lal 13481 (LWG); iii.1963, Rampurwa, Nepal Border, G. Panigrahi 2898 (BSA); 18.iv.2003, coll. S.D. Maliya 224925 (LWG); 22.iv.2003, Nishangara range, coll. S.D. Maliya 214819 (LWG) (Image 24).

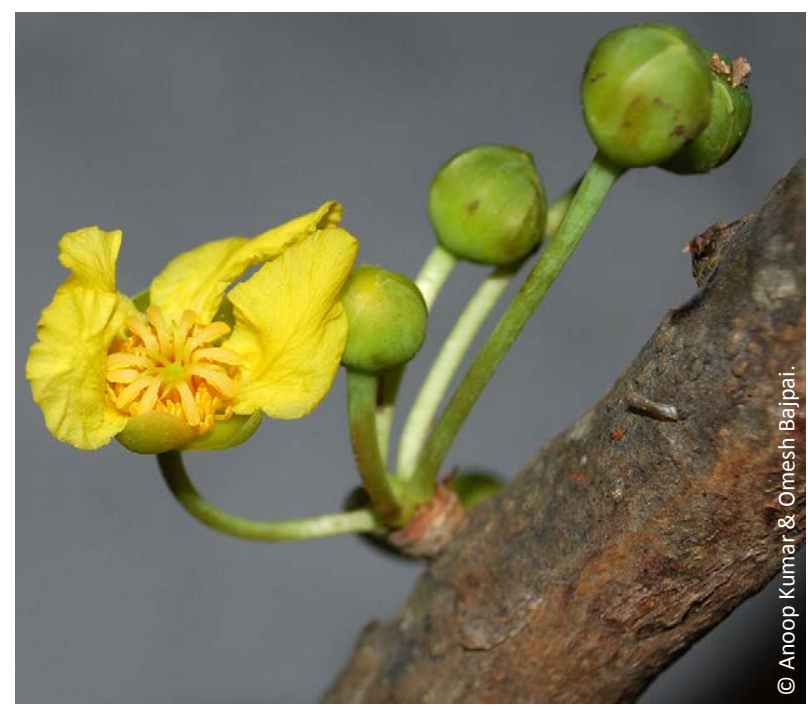

Image 23. Dillenia pentagyna Roxb.

\section{EBENACEAE}

Diospyros exculpta Buch.-Ham., Trans. Linn. Soc. London 15: 110. 1826.

Diospyros tomentosa Roxb., Fl. Ind. 2: 532. 1832.

Habit: Tree.

Specimens examined: 27.iv.2010, Balcha chowki, Kakraha range, L.B. Chaudhary, A. Kumar \& O. Bajpai 252247 (LWG) (Image 25).

Diospyros melanoxylon Roxb., PI. Coromandel 1: 36. 1795.

Habit: Tree.

Specimens examined: 22.v.1986, Dharmapur, K.K. Khanna \& party 37104 (BSA).

\section{EUPHORBIACEAE}

Acalypha indica L., Sp. PI. 1003. 1753; Hook. f. in Hook. f., Fl. Brit. India 5: 416. 1887; Duthie, Fl. Upp. Gang. Plain 3: 209. repr. ed. 1960; Maliya \& Datt, J. Econ. Taxon. Bot. 34 (1): 62.2010.

Habit: Annual herb.

Specimens examined: 22.xii.2003, Nishangara range, coll. S.D. Maliya 225236 (LWG).

Chrozophora tinctoria (L.) A. Juss., Euphorb. gen. 28, 110, t. 7. 1824 (Rafinesque, Chlor. aetn. 4. 1813, nom. inval.

Croton tinctorius L., Sp. PI. 1004. 1753.

Habit: Herb.

Specimens examined: 27.v.1954, Motipur range, Hira Lal 13467 (LWG) (Image 26).

Croton bonplandianus Baill., Adansonia 4: 339. 1864; Raizada, Suppl. Fl. Upp. Gang. Plain 247. 1976; Maliya \&

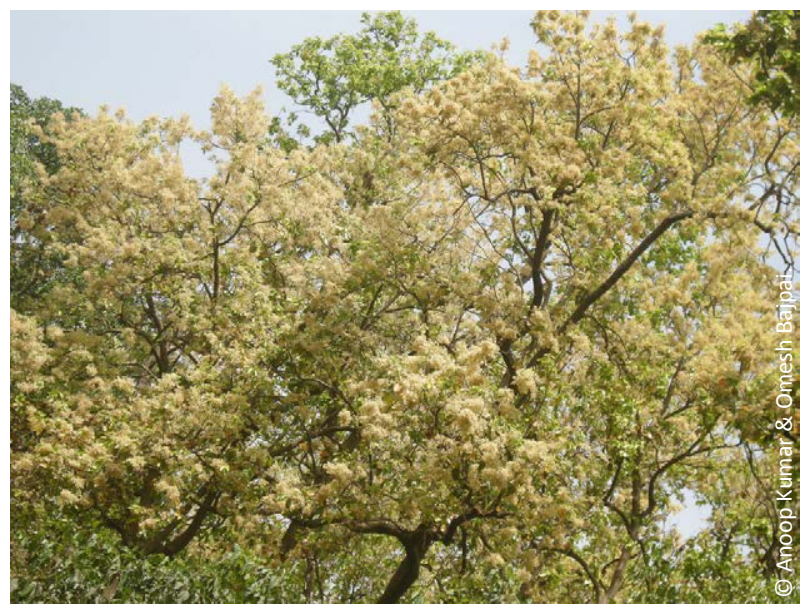

Image 24. Shorea robusta Gaertn. f. 


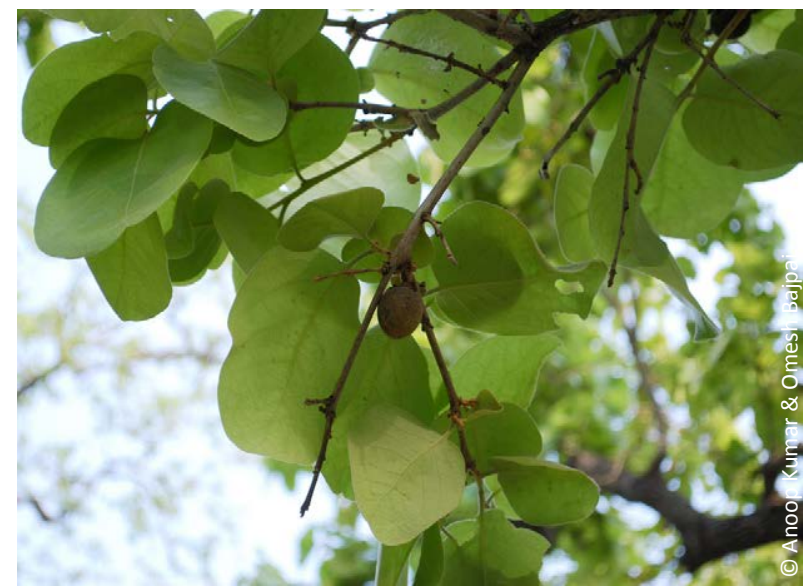

Image 25. Diospyros exculpta Buch.-Ham.

Datt, J. Econ. Taxon. Bot. 34 (1): 62.2010.

C. sparsiflorus Morong., Ann. N. Y. Acad. Sci. 7: 221. 1893.

Habit: Herb or undershrub.

Specimens examined: 25.viii.2002, Murtiha range, coll. S.D. Maliya 223728 (LWG).

Euphorbia helioscopia L., Sp. PI. 459. 1753; Hook. f. in Hook. f., Fl. Brit. India 5: 262. 1887; Raizada, Suppl. Fl. Upp. Gang. Plain 248. 1976; Maliya \& Datt, J. Econ. Taxon. Bot. 34 (1): 62.2010. 1812.

Galarhoeus helioscopius (L.) Haw., Syn. PI. Succ. 152.

Habit: Herb.

Specimens examined: 20.iii.2004, Girijapuri forest, coll. S.D. Maliya 225930 (LWG).

Euphorbia hirta L., Sp. PI. 454. 1753; Duthie, Fl. Upp. Gang. Plain 3: 184. repr. ed. 1960; Saini, J. Econ. Taxon. Bot. 29 (4): 872. 2005; Maliya \& Datt, J. Econ. Taxon. Bot. 34 (1): 62.2010.

Chamaesyce hirta (L.) Millsp., Publ. Field Columb. Mus. Nat. Hist. Bot. ser. 2 (7): 303. 1909.

Habit: Annual herb.

Specimens examined: 23.viii.2002, Nishangara range, coll. S.D. Maliya 223628 (LWG); 25.viii.2002, rest house, Murtiha range, coll. S.D. Maliya 223751 (LWG); 25.viii.2002, Murtiha range, coll. S.D. Maliya 223740 (LWG); 29.viii.2002, Murtiha range, coll. S.D. Maliya 223800 (LWG); 09.xi.2002, road side, Katerniaghat range, coll. S.D. Maliya 224544 (LWG); 16.xii.2004, Kuthiyaghat forest, coll. S.D. Maliya 225951 (LWG).

Euphorbia neriifolia L., Sp. PI. 451. 1753; Duthie, FI. Upp. Gang. Plain 3: 184. repr. ed. 1960; Maliya \& Datt, J.

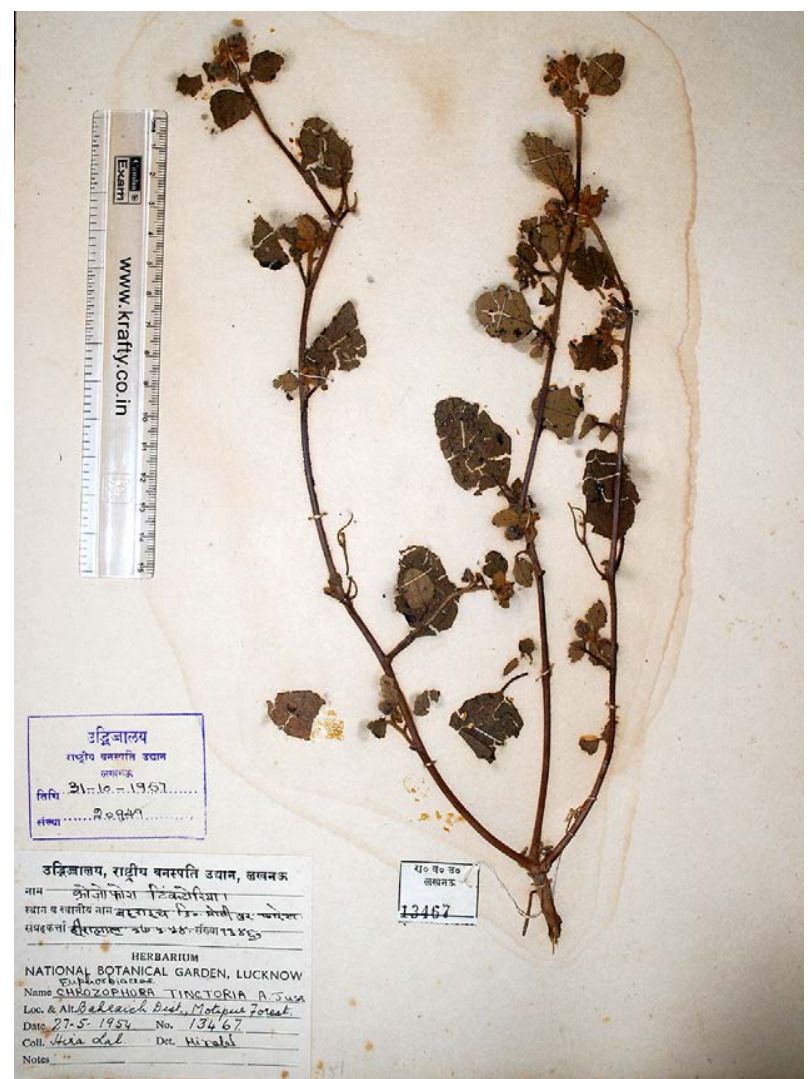

Image 26. Chrozophora tinctoria (L.) A. Juss.

Econ. Taxon. Bot. 34 (1): 62.2010.

E. ligularia Roxb. ex Buch. - Ham., Trans. Linn. Soc. London 14: 285. 1825.

Habit: Shrub or small tree.

Specimens examined: 31.viii.2003, Fakirpuri, Rampurva village, coll. S.D. Maliya 214889 (LWG).

Euphorbia parviflora L., Syst. Nat. 2: 1047. 1759, nom. cons. prop.; Hook. f. in Hook. f., Fl. Brit. India 5: 250. 1887; Panigrahi et al., Bull. Bot. Surv. India 11 (1 \& 2): 99. 1969.

E. pilulifera L., Sp. PI. 454. 1753, p.p. as to lectotype, nom. rejic. prop. Chamaesyce pilulifera (L.) Small, FI. SE. U.S. 708. 1903.

Habit: Annual herb.

Specimens examined: Not observed. Record based on Panigrahi (1969).

*Euphorbia pulcherrima Willd. ex Klotzsch, Allg. Gartenzeitung 2: 27. 1834; Saini, J. Econ. Taxon. Bot. 29 (4): 873. 2005.

Habit: Shrub, cultivated.

Specimens examined: Not observed. Record based on Saini (2005). 
**Jatropha curcas L., Sp. PI. 1006. 1753; Hook. f. in Hook. f., Fl. Brit. India 5: 383. 1887; Duthie, Fl. Upp. Gang. Plain 3: 215. repr. ed. 1960; Panigrahi et al., Bull. Bot. Surv. India 11 (1 \& 2): 99. 1969; Maliya \& Datt, J. Econ. Taxon. Bot. 34 (1): 62.2010.

Habit: Shrub or small tree.

Specimens examined: 16.vii.2004, Kuthiyaghat forest, coll. S.D. Maliya 225955 (LWG); 24.xii.2004, Kuthiyaghat forest, coll. S.D. Maliya 225256 (LWG).

Mallotus nudiflorus (L.) Kulju \& Welzen, Blumea 52: 124. 2007.

Trewia nudiflora L., Sp. Pl. 1193. 1753; Duthie, Fl. Upp. Gang. Plain 3: 210. repr. ed. 1960; Maliya \& Datt, J. Econ. Taxon. Bot. 34 (1): 63. 2010. T. polycarpa Benth., Gen. PI. 3: 318. 1880; Hook. f. in Hook. f., Fl. Brit. India 5: 424. 1887; Saini, J. Econ. Taxon. Bot. 29(5): 876. 2005.

Habit: Tree.

Specimens examined: 26.v.1954, Motipur range, Hira Lal 13426 (LWG); 14.iv.1967, S. L. Kapoor \& party 70041 (LWG); 21.v.1987, K.K. Singh \& party 5975 (LWG); 24.xi.1987, Nishangara range, K.K. Singh \& party 6195 (LWG); 26.viii.2002, Katerniaghat range, coll. S.D. Maliya 223756 (LWG); 20.iv.2003, Murtiha range, coll. S.D. Maliya 224948 (LWG); 28.viii.2003, Murtiha range, coll. S.D. Maliya 214851 (LWG); 22.iv.2003, Nishangara range, coll. S.D. Maliya 224994 (LWG); 28.viii.2003, Murtiha range, coll. S.D. Maliya 214851 (LWG); 16.iii.2004, Karmohini forest, coll. S.D. Maliya 225284 (LWG) (Image 27).

Mallotus philippensis (Lam.) Muell.-Arg., Linnaea, 34: 196. 1865; Hook. f. in Hook. f., Fl. Brit. India 5: 442. 1887; Duthie, Fl. Upp. Gang. Plain 3: 210. repr. ed. 1960; Maliya \& Datt, J. Econ. Taxon. Bot. 34 (1): 62.2010.

Croton philippensis Lam., Encycl. 2: 206. 1786.

Habit: Tree.

Specimens examined: 17.v.1987, K.K. Singh \& party 5950 (LWG); 24.xi.1987, Nishangara range, K.K. Singh 6179 (LWG); 23.viii.2002, Nishangara range, coll. S.D. Maliya 223659 (LWG); 09.xi.2002, near Bichhiya, coll. S.D. Maliya 224502 (LWG); 10.xi.2002, Murtiha range, coll. S.D. Maliya 224595 (LWG); 11.xi.2002, Murtiha range, coll. S.D. Maliya 224601 (LWG); 12.xi.2002, Nishangara range, coll. S.D. Maliya 224659 (LWG); 16.iii.2008, Murtiha range, coll. S.D. Maliya 218509 (LWG) (Image 28).

Ricinus communis L., Sp. PI. 1007.1753.

Habit: Shrub.

Specimens examined: 21.v.1987, K.K. Singh \& party 5981 (LWG) (Image 29).

\section{FABACEAE (CAESALPINIOIDEAE)}

Bauhinia acuminata L., Sp. PI. 375. 1753; Baker in Hook. f., Fl. Brit. India 2: 276. 1879.

Habit: Tree.

Specimens examined: 31.x.2009, Sujauli road, near oripurwa, L.B. Chaudhary, A. Kumar \& O. Bajpai, 252172(LWG).

Bauhinia malabarica Roxb., Fl. Ind. 2: 321. 1832; Baker in Hook. f., Fl. Brit. India 2: 277. 1879; Maliya \& Datt, J. Econ. Taxon. Bot. 34 (1): 51. 2010.

Piliostigma malabarica (Roxb.) Benth. in Miq., PI. Jungh. 261. 1852; Panigrahi et al., Bull. Bot. Surv. India 11 (1 \& 2): 94.1969.

Habit: Tree.

Specimens examined: 21.xi.1964, Nishangara range, G. Pannigrahi \& O.P. Mishra 6490 (BSA); 11.ii.1965, Bichhiya, O.P. Mishra 7935 (BSA); 16.ii.1965, Katerniaghat mandi, O.P. Mishra 8024 (BSA); 08.xii.1986, Nishangara

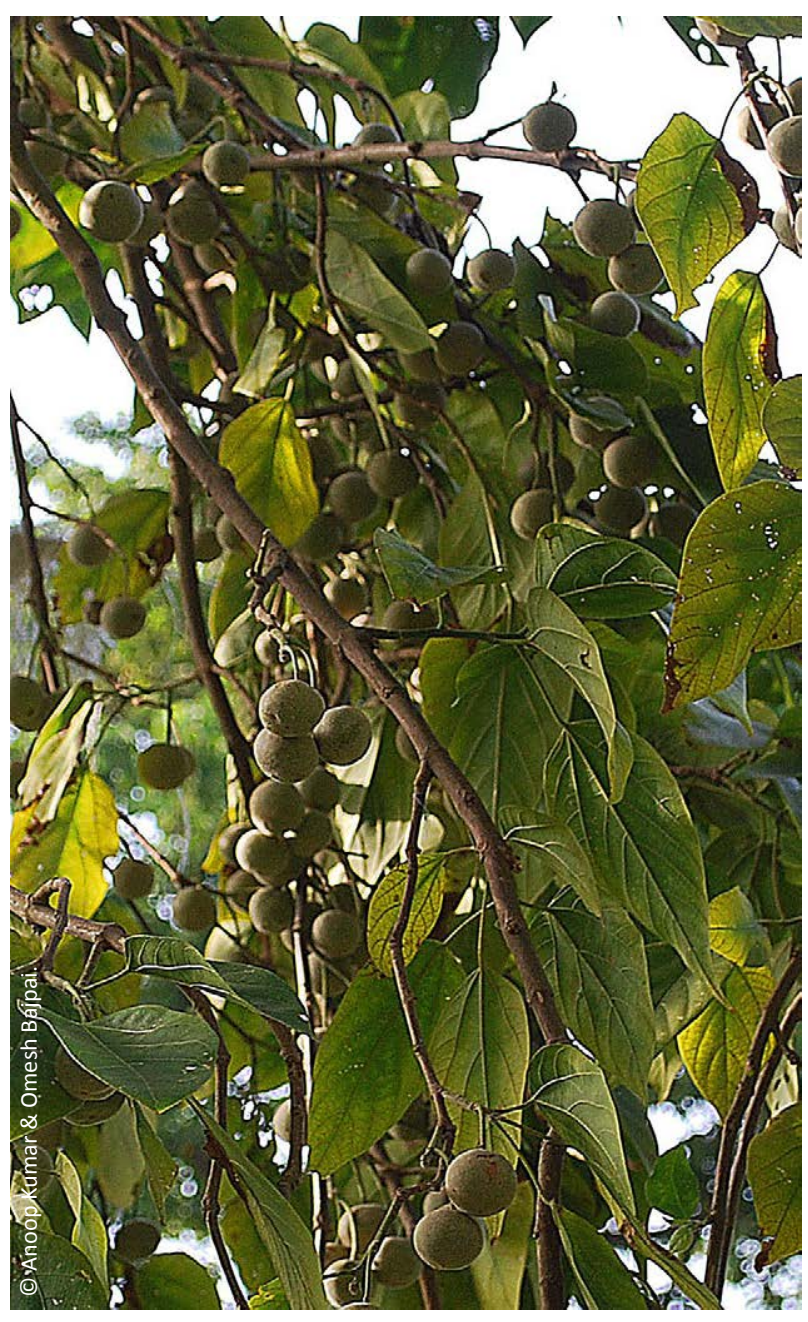

Image 27. Mallotus nudiflorus (L.) Kulju \& Welzen 


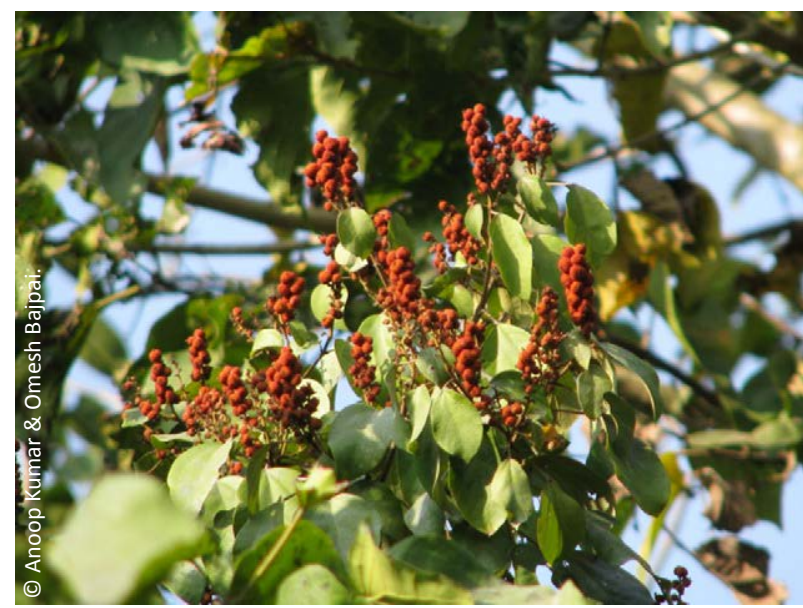

Image 28. Mallotus philippensis (Lam.) Muell.-Arg.

range, K.K. Khanna \& R. Saran 37604 (BSA); 11.xi.2002, road side, Murtiha range, coll. S.D. Maliya 224617 (LWG); 22.iv.2003, Nishangara range, coll. S.D. Maliya 224983 (LWG).

Bauhinia purpurea L., Sp. Pl. 375. 1753; Baker in Hook. f., Fl. Brit. India 2: 284. 1879; Duthie, Fl. Upp. Gang. Plain 1: 218. repr. ed. 1960.

B. triandra Roxb., Fl. Ind. 2: 320. 1832. Phanera purpurea (L.) Benth. in Miq., Pl. Jungh. 262. 1852.

Habit: Tree.

Specimens examined: 28.x.2009, In front of Kakraha rest house, L.B. Chaudhary, A. Kumar \& O. Bajpai 252140 (LWG).

*Bauhinia roxburghiana Voigt, Hort. Suburb. Calc. 254. 1845; Larsen \& Larsen, Taxon 28: 591. 1979 pp.; Saini, J. Econ. Taxon. Bota. 29 (3): 569. 2005.

B. retusa Ham. ex Roxb., Fl. Ind. 2: 322. 1832; Baker in Hook. f., Fl. Brit. India 2: 279. 1879.

Habit: Tree, cultivated.

Specimens examined: Not observed. Record based on Saini (2005).

Bauhinia vahlii Wight. \& Arn., Prodr. Fl. India Orient. 1: 297. 1834; Baker in Hook. f., Fl. Brit. India 2: 279. 1879 ; Saini, J. Econ. Taxon. Bot. 29 (3): 569. 2005; Maliya \& Datt, J. Econ. Taxon. Bot. 34 (1): 51. 2010.

Phanera vahlii (Wight. \& Arn.) Benth. in Miq., PI. Jungh. 263. 1852.

Habit: Climber.

Specimens examined: 16.v.1987, K.K. Singh \& party 5990 (LWG) (Image 30).

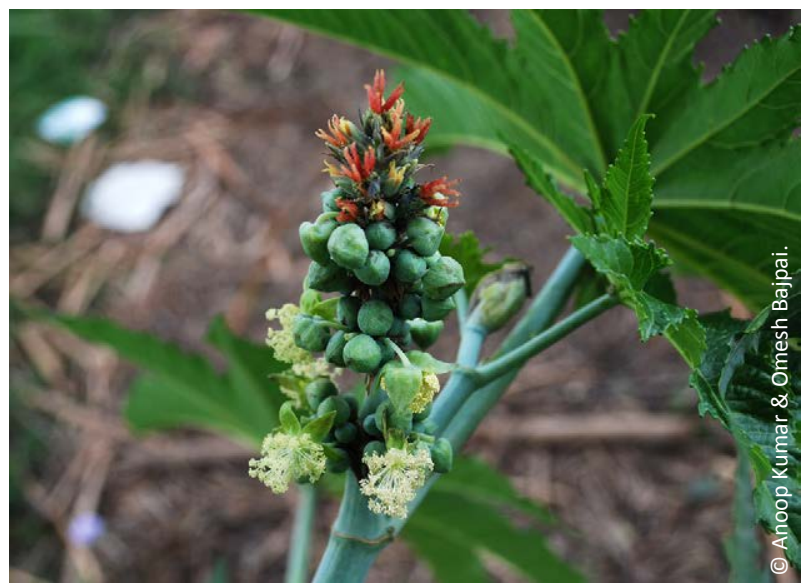

Image 29. Ricinus communis L.

Bauhinia variegata L., Sp. pl. 1:375. 1753; Maliya, J. Econ. Taxon. Bot. 36 (2): 420. 2012.

Habit: Tree.

Specimens examined: 25.i.2005, Kuthiyaghat, coll. S.D. Maliya 224688 (LWG).

Caesalpinia bonduc (L.) Roxb., Fl. Ind. 2: 362. 1832; Maliya \& Datt, J. Econ. Taxon. Bot. 34 (1): 51. 2010.

Guilandina bonduc L., Sp. PI. 1:381. 1753.

Habit: Scandent shrub.

Specimens examined: Not observed. Record based on Maliya \& Datt (2010).

Caesalpinia crista L., Sp. PI. 380. 1753 pp; Panigrahi et al., Bull. Bot. Surv. India 11 (1 \& 2): 94.1969.

Habit: Scandent shrub.

Specimens examined: 22.xi.1964, Orla, Motipur range, G. Panigrahi \& O.P. Mishra 6586 (BSA).

Caesalpinia cucullata Roxb., Fl. Ind. 2: 358. 1832; Hattink, Reinwardtia 9: 22. 1974; Saini, J. Econ. Taxon. Bot. 29 (3): 569. 2005.

Mezoneurum cucullatum (Roxb.) Wight \& Arn., Prodr. FI. India Orient. 283. 1834; Baker in Hook. f., FI. Brit. India 2: 258.1879.

Habit: Climber.

Specimens examined: Not observed. Record based on Saini (2005).

Cassia fistula L., Sp. PI. 377. 1753; Baker in Hook. f., Fl. Brit. India 2: 261. 1879; Duthie, Fl. Upp. Gang. Plain 1: 268. repr. ed. 1960; Saini, J. Econ. Taxon. Bota. 29 (3): 570. 2005; Maliya \& Datt, J. Econ. Taxon. Bot. 34 (1): 52. 2010. 


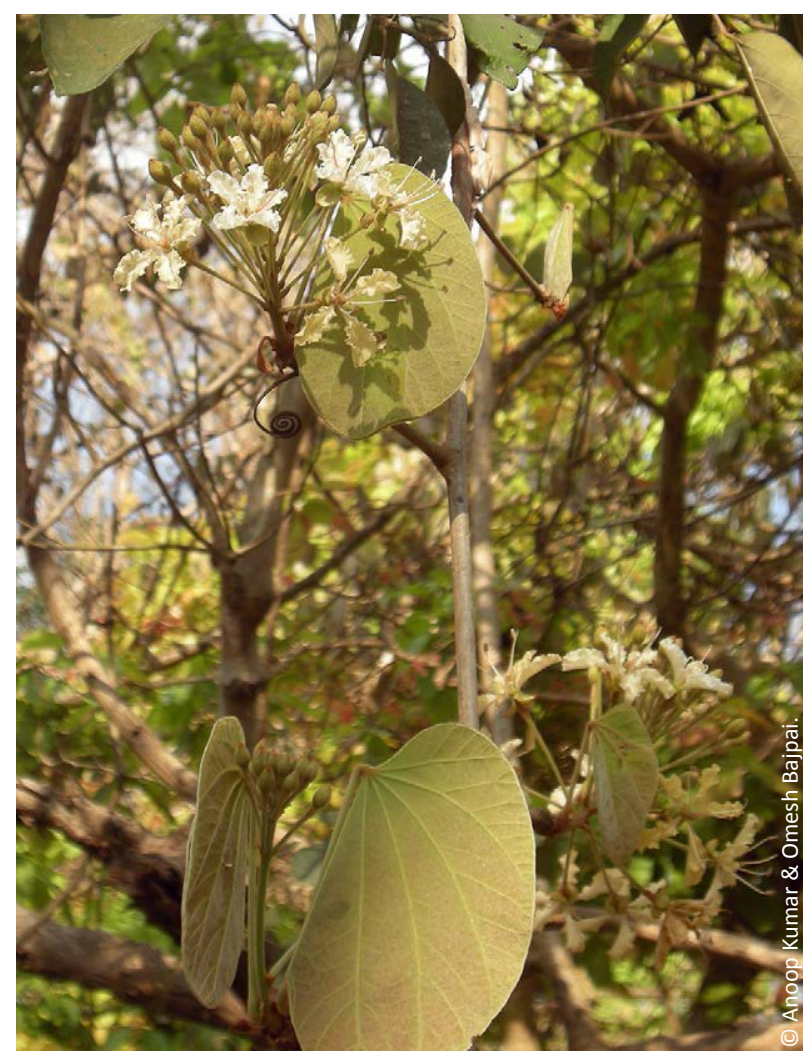

Image 30. Bauhinia vahlii Wight. \& Arn.

Habit: Tree.

Specimens examined: 26.viii.2002, Katerniaghat range, coll. S.D. Maliya 22753 (LWG); 10.xi.2002, Murtiha range, coll. S.D. Maliya 224593 (LWG).

Chamaecrista absus (L.) Irwin \& Barneby, Mem. New York Bot. Gard. 35:664. 1982; Maliya \& Datt, J. Econ. Taxon. Bot. 34 (1): 52. 2010.

Cassia absus L., Sp. PI. 376. 1753; Baker in Hook. f., FI. Brit. India 2: 265. 1876.

Habit: Herb.

Specimens examined: 11.xi.2002, Murtiha range, coll. S.D. Maliya 224612 (LWG).

**Chamaecrista nictitans (L.) Moench., Methodus, 272. 1794.

Cassia nictitans L., Sp. PI. 380. 1753. C. patellaria var. glabrata Vogel, Gen. Cass. Syn. 66. 1837. C. mimosoides subsp. leschenaultiana (DC.) H. Ohashi, J. Jap. Bot., 50(10): 308. 1975. C. lechenaultiana DC., Mém. Soc. Phys. Genève 2(2): 132. 1824.

Habit: Herb.

Specimens examined: 09.ix.2005, rest house, Murtiha range, coll. S.D. Maliya 227170 (LWG).
*Delonix regia (Bojer ex Hook.) Raf., Fl. Tell. 2: 92. 1837; Maliya \& Datt, J. Econ. Taxon. Bot. 34 (1): 52. 2010.

Poinciana regia Bojer ex Hook., Bot. Mag. 56: t. 2884. 1829.

Habit: Tree, cultivated.

Specimens examined: 26.viii.2002, Katerniaghat range, coll. S.D. Maliya 223783 (LWG).

*Parkinsonia aculeata L., Sp. PI. 375. 1753; Baker in Hook. f., Fl. Brit. India 2: 260. 1879; Duthie, Fl. Upp. Gang. Plain 1: 280. repr. ed. 1960.

Habit: Tree, cultivated.

Specimens examined: 26.vii.2009, Near Balcha barrier, Kakraha range, L.B. Chaudhary, A. Kumar \& O. Bajpai 250678 (LWG).

Senna occidentalis (L.) Link, Handbuch 2: 140. 1829.

Cassia occidentalis L., Sp. PI. 377. 1753; Baker in Hook. f., Fl. Brit. India 2: 262. 1879; Panigrahi et al., Bull. Bot. Surv. India 11 (1 \& 2): 94.1969.

Habit: Undershrub or herb.

Specimens examined: 21.xi.1964, Nishangara range, O.P. Mishra 6491 (BSA); 08.xii.1986, Girijapuri forest, K.K. Khanna \& R. Saran 37654 (BSA).

Senna siamea (Lam.) Irwin \& Barneby, Mem. New York Bot. Gard. 35: 98. 1982; Panigrahi et al., Bull. Bot. Surv. India 11 (1 \& 2): 94. 1969; Maliya \& Datt, J. Econ. Taxon. Bot. 34 (1): 52. 2010.

Cassia siamea Lam., Encycl. 1: 648. 1785; Baker in Hook. f., Fl. Brit. India 2: 264. 1879; Saini, J. Econ. Taxon. Bota. 29 (3): 571.2005.

Habit: Tree.

Specimens examined: 08.ii.1965, Kauriala, O.P. Mishra 7751 (BSA); 08.xii.1986, Bichhiya, K.K. Khanna 37651 (BSA); 14.xi.2002, Murtiha range, coll. S.D. Maliya 224679 (LWG).

Senna sophera (L.) Roxb., Fl. Ind. 2: 347. 1832.

Cassia sophera L., Sp. PI. 379. 1753; Baker in Hook. f., FI. Brit. India 2: 262. 1879; Saini, J. Econ. Taxon. Bota. 29 (3): 571.2005.

Habit: Shrub or undershrub.

Specimens examined: Not observed. Record based on Saini (2005).

Senna tora (L.) Roxb., Fl. Ind. 2: 340. 1832; Maliya \& Datt, J. Econ. Taxon. Bot. 34 (1): 52. 2010.

Cassia tora L., Sp. PI. 376. 1753; Baker in Hook. f., Fl. Brit. India 2: 263. 1879.

Habit: Annual herb. 
Specimens examined: 25.viii.2002, Murtiha range, coll. S.D. Maliya 223712 (LWG).

Tamarindus indica L., Sp. PI. 34. 1753; Baker in Hook. f., Fl. Brit. India 2: 273. 1879; Duthie, Fl. Upp. Gang. Plain 1: 277. repr. ed. 1960; Saini, J. Econ. Taxon. Bota. 29 (3): 572. 2005.

Habit: Tree.

Specimens examined: Not observed. Record based on Saini (2005)

\section{FABACEAE (MIMOSOIDEAE)}

*Acacia auriculiformis A. Cunn. ex Benth. in Hook., London J. Bot. 1: 377. 1842; Maliya \& Datt, J. Econ. Taxon. Bot. 34 (1): 52. 2010.

Racosperma auriculiforme (A. Cunn. ex Benth.) Pedley, Bot. J. Linn. Soc. 92: 247. 1986.

Habit: Tree, cultivated.

Specimens examined: 27.x.2004, Katerniaghat range, coll. S.D. Maliya 227129 (LWG).

Acacia catechu (L. f.) Willd., Sp. Pl. 4: 1079. 1806; Baker in Hook. f., Fl. Brit. India 2: 295. 1879; Duthie, Fl. Upp. Gang. Plain 1: 290. repr. ed. 1960; Panigrahi et al., Bull. Bot. Surv. India 11 (1 \& 2): 94. 1969; Maliya \& Datt, J. Econ. Taxon. Bot. 34 (1): 52. 2010.

Mimosa catechu L. f., Suppl. PI. 439. 1782.

Habit: Tree.

Specimens examined: 21.xi.1964, Nishangara range, G. Panigrahi \& O.P. Mishra 6515 (BSA); 23.viii.2002, Nishangara range, coll. S.D. Maliya 223634 (LWG); 09.xi.2002, on road, Katerniaghat range, coll. S.D. Maliya 224537 (LWG); 02.ix.2003, Kuthiyaghat forest, coll. S.D. Maliya 225208 (LWG) (Image 31).

Acacia concinna (Willd.) DC., Prodr. 2: 264. 1825; Baker in Hook. f., Fl. Brit. India 2: 296. 1879.

Mimosa concinna Willd., Sp. PI. 1039. 1806. Acacia rugata Lam., Encycl. 1: 20. 1783. Mimosa rugata Lam., Encycl. 1(1): 20. 1783. Mimosa sinuata Lour., Fl. Cochinch. 2: 653. 1790. Acacia rugata (Lam.) Buch. - Ham. ex Benth. in Hook., London J. Bot. 1: 514. 1842. Acacia concinna DC. var. rugata (Buch.-Ham. ex Benth.) Baker in Hook. f., Fl. Brit. India 2: 297. 1879; Panigrahi et al., Bull. Bot. Surv. India 11 (1 \& 2): 94. 1969. Acacia sinuata (Lour.) Merr., Trans. Amer. Philos. Soc. n. s. 24(2): 186. 1935; Maliya \& Datt, J. Econ. Taxon. Bot. 34 (1): 52. 2010.

Habit: Climbing shrub.

Specimens examined: 11.ii.1965, Bichhiya, Motipur range, O.P. Mishra 7933 (BSA); 24.xi.1987, Nishangara range, K.K. Singh \& team 6180 (LWG); 20.iv.2003, Murtiha range, coll. S.D. Maliya 224945 (LWG); 22.iv.2003, Nishangara range, coll. S.D. Maliya 214817 (LWG); 31.viii.2004, Amba forest coll. S.D. Maliya 214890 (LWG); 22.x.2005, Saryu link canal, Mihinpurwa road, coll. S.D. Maliya 227177 (LWG) (Image 32).

Acacia nilotica (L.) Willd. ex Delile, Descr. Egypte, Hist. Nat. 79. 1813.

Mimosa nilotica L., Sp. PI. 521. 1753. Acacia arabica (Lam.) Willd., Sp. PI. 4(2): 1085. 1806. Mimosa arabica Lam., Encycl. 1 (1): 19. 1783.

Habit: Tree.

Specimens examined: 31.x.2009, Sujauli road, Katerniaghat range, L.B. Chaudhary, A. Kumar \& O. Bajpai 252169 (LWG).

Acacia pennata (L.) Willd., Sp. PI. 1090. 1806; Panigrahi et al., Bull. Bot. Surv. India 11 (1 \& 2): 94.1969.

Mimosa pennata L., Sp. PI. 522. 1753.

Habit: Scandent bush or creeper.

Specimens examined: Not observed. Record based on Panigrahi et al. (1969).

Albizia chinensis (Osbeck) Merr., Amer. J. Bot. 3: 575. 1916; Ali, Fl. W. Pak. 36: 21. 1973; Saini, J. Econ. Taxon. Bot. 29 (3): 573. 2005.

Mimosa chinensis Osbeck, Dagb. Ostind. Resa 233. 1757. Acacia stipulata DC., Prodr. 2: 469. 1825. Albizia stipulata (DC.) Boiv., Encycl. 19. Siecle 2: 33. 1838; Baker in Hook. f., Fl. Brit. India 2: 300. 1879. pp.

Habit: Tree.

Specimens examined: Not observed. Record based on Saini (2005).

Albizia lebbeck (L.) Benth., Hook. London J. Bot. 3: 87. 1844; Baker in Hook. f., Fl. Brit. India 2: 298. 1879; Duthie,

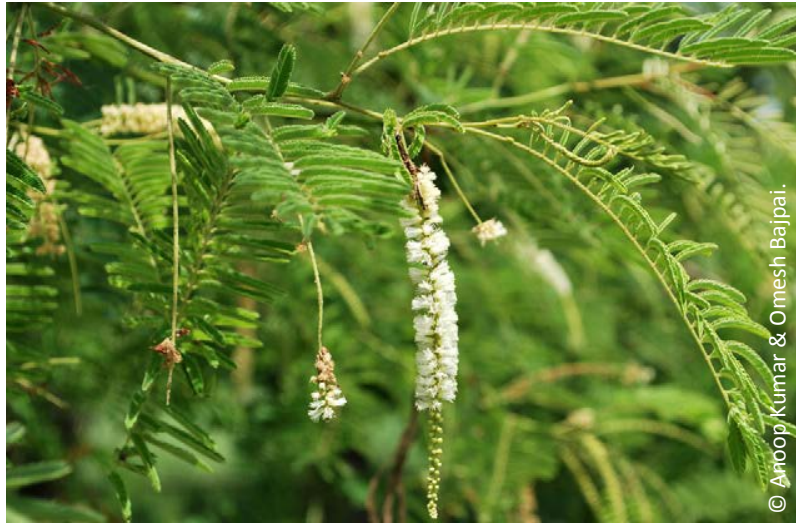

Image 31. Acacia catechu (L. f.) Willd. 


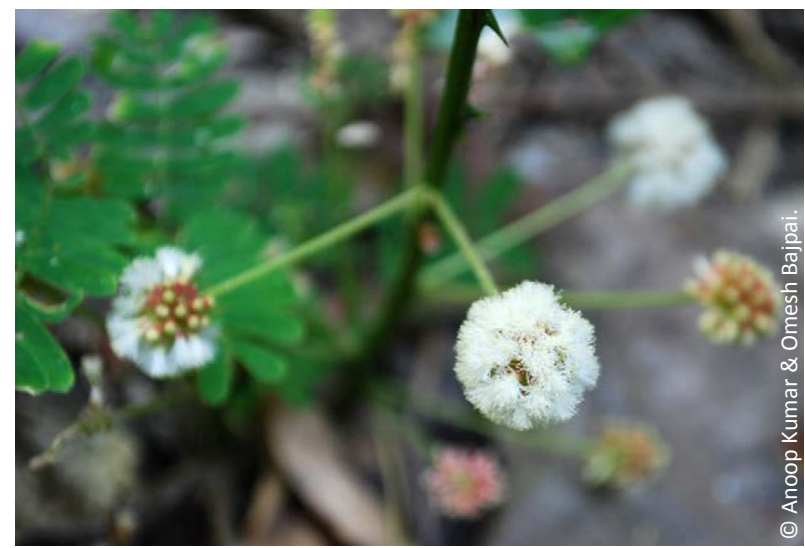

Image 32. Acacia concinna (Willd.) DC.

Fl. Upp. Gang. Plain 1: 295. repr. ed. 1960; Maliya \& Datt, J. Econ. Taxon. Bot. 34 (1): 52. 2010.

Mimosa lebbeck L., Sp. PI. 516. 1753.

Habit: Tree.

Specimens examined: 11.iv,2009, Barsha fata area, L.B. Chaudhary 250770 (LWG); 28.x.2009, near rest house, Kakraha range, L.B. Chaudhary, A. Kumar \& O. Bajpai 252141 (LWG).

Albizia odoratissima (L. f.) Benth., London J. Bot. 3: 88. 1844; Baker in Hook. f., Fl. Brit. India 2: 299. 1879; Nielsen, Adansonia 19 (2): 225. 1979; Saini, J. Econ. Taxon. Bot. 29 (3): 573. 2005.

Mimosa odoratissima L. f., Suppl. PI. 437. 1781. Albizia odoratissma var. mollis Benth. ex Baker in Hook. f., Fl. Brit. India 2: 299. 1879.

Habit: Tree.

Specimens examined: Not observed. Record based on Saini (2005).

Albizia procera (Roxb.) Benth. in Hook., London J. Bot. 3: 89. 1844; Saini, J. Econ. Taxon. Bot. 29 (3): 574. 2005; Maliya \& Datt, J. Econ. Taxon. Bot. 34 (1): 52. 2010.

Mimosa procera Roxb., PI. Coromandel 2: 12, t. 121. 1798.

Habit: Tree.

Specimens examined: 20.iv.2003, Murtiha range, coll. S.D. Maliya 224950 (LWG); 21.iv.2003, Nishangara range, coll. S.D. Maliya 224967 (LWG); 24.xii.2003, Kuthiyaghat forest, coll. S.D. Maliya 225250 (LWG); 22.x.2004, Kuthiyaghat forest, coll. S.D. Maliya 225986 (LWG).

**Leucaena leucocephala (Lam.) de Wit., Taxon 10: 54. 1961; Panigrahi et al., Bull. Bot. Surv. India 11 (1 \& 2): 94. 1969.

Mimosa leucocephala Lam., Encycl. 1: 12. 1783.
Lysiloma latisiliquum sensu Maliya \& Datt, J. Econ. Taxon. Bot. 34 (1): 52. 2010.

Habit: Tree.

Specimens examined: 27.viii.2003, near railway station, Bichhiya town, coll. S.D. Maliya 214849 (LWG).

Mimosa himalayana Gamble, Bull. Misc. Inform. Kew 1920: 4. 1920; Panigrahi et al., Bull. Bot. Surv. India 11 (1 \& 2): 94.1969.

M. rubicaulis Lam. subsp. himalayana (Gamble) Ohashi, Enum. FI. PI. Nepal. 2: 126. 1979; Saini, J. Econ. Taxon. Bot. 29 (3): 574. 2005.

Habit: Shrub.

Specimens examined: 21.xi.1964, Nishangara range, G. Panigrahi \& O.P. Mishra 6489 (BSA); 07.xii.1986, Motipur, K.K. Khanna \& R. Saran 38587 (BSA); 25.viii.2002, Murtiha range, coll. S.D. Maliya 223694 (LWG); 23.iii.2004, Kuthiyaghat forest, coll. S.D. Maliya 225938 (LWG).

**Mimosa pudica L., Sp. Pl. 518. 1753; Baker in Hook. f., Fl. Brit. India 2; 291. 1879; Maliya \& Datt, J. Econ. Taxon. Bot. 34 (1): 52. 2010.

Habit: Herb or undershrub.

Specimens examined: Not observed. Record based on Maliya \& Datt (2010).

\section{fabaceae (PAPilionoideae)}

Abrus precatorius L., Syst. Nat. 472. 1767; Baker in Hook. f., FI. Brit. India 2: 175. 1879; Duthie, Fl. Upp. Gang. Plain 1: 241. repr. ed. 1960; Saini, J. Econ. Taxon. Bot. 29 (3): 557. 2005; Maliya \& Datt, J. Econ. Taxon. Bot. 34 (1): 50. 2010.

Glycine abrus L., Sp. PI. 753. 1753.

Habit: Woody climber.

Specimens examined: 13.xi.2002, Nishangara range, coll. S.D. Maliya 224668 (LWG); 22.iv.2003, Nishangara range, coll. S.D. Maliya 224986 (LWG); 05.xi.2008, Katerniaghat range, coll. S.D. Maliya 226642 (LWG).

Aeschynomene indica L., Sp. PI. 713. 1753; Baker in Hook. f., Fl. Brit. India 2: 151. 1879; Duthie, Fl. Upp. Gang. Plain 1: 249. repr. ed. 1960; Saini, J. Econ. Taxon. Bot. 29 (3): 558. 2005.

A. pumila L., Sp. PI. 1061. 1763.

Habit: Annual herb.

Specimens examined: Not observed. Record based on Saini (2005).

Alysicarpus bupleurifolius (L.) DC., Prodr. 2: 352. 1825; Baker in Hook. f., FI. Brit. India 2: 158. 1879; Duthie, FI. Upp. Gang. Plain 1: 255. repr. ed. 1960; Maliya \& Datt, 
J. Econ. Taxon. Bot. 34 (1): 50. 2010.

Hedysarum bupleurifolium L., Sp. PI. 745. 1753.

Habit: Herb.

Specimens examined: 09.xi.2004, road side, Katerniaghat range, coll. S.D. Maliya 224555 (LWG); 22.x.2010, Nishangara range, coll. S.D. Maliya 252622 (LWG).

Alysicarpus longifolius (Rottl. ex Spreng.) Wight. \& Arn., Prodr. Fl. Ind. Orient. 1: 233. 1834; Baker in Hook. f., Fl. Brit. India 2: 159. 1879; Maliya \& Datt, J. Econ. Taxon. Bot. 34 (1): 50.2010

Hedysarum longifolium Rottl. ex Spreng., Syst. Veg. 3: 319. 1826.

Habit: Herb.

Specimens examined: 24.viii.2002, Nishangara range, coll. S.D. Maliya 223689 (LWG).

Alysicarpus vaginalis (L.) DC., Prodr. 2: 353. 1825; Baker in Hook. f., Fl. Brit. India 2: 158. 1879; Duthie, FI. Upp. Gang. Plain 1: 255. repr. ed. 1960; Saini, J. Econ. Taxon. Bot. 29 (3): 558. 2005.

Hedysarum vaginale L., Sp. PI. 747. 1753.

Habit: Undershrub.

Specimens examined: Not observed. Record based on Saini (2005)

**Arachis hypogaea L., Sp. PI. 741. 1753; Baker in Hook. f., Fl. Brit. India 2: 161. 1879; Panigrahi et al., Bull. Bot. Surv. India 11 (1 \& 2): 94. 1969.

Habit: Herb.

Specimens examined: Not observed. Record based on Panigrahi et al. (1969).

Butea monosperma (Lam.) Taub. in Engl. \& Prantl. Nat. Pflanzen. 3 (3): 366. 1894; Hook. f. Fl. Brit. India 2: 194. 1876; Duthie, Fl. Upp. Gang. Plain 1: 221. repr. ed. 1960; Panigrahi et al., Bull. Bot. Surv. India 11 (1 \& 2): 95. 1969; Maliya \& Datt, J. Econ. Taxon. Bot. 34 (1): 50. 2010.

Erythrina monosperma Lam., Encycl. 2: 391. 1786. Butea frondosa Roxb. ex Willd., Sp. PI. 917. 1802; Baker in Hook. f., Fl. Brit. India 2: 194. 1879.

Habit: Tree.

Specimens examined: 15.iii.1964, Rampurwa, G. Panigrahi \& O.P. Mishra 2911 (BSA); 11.ii.1965, Bichhiya, O.P. Mishra 7930 (BSA); 02.ix.2003, Girijapuri forest, coll. S.D. Maliya 214897 (LWG); 20.iii.2004, Girijapuri forest, coll. S.D. Maliya 225919 (LWG).

*Cajanus cajan (L.) Huth., Helios 11:133. 1893 'Cajan cajan'; Maliya \& Datt, J. Econ. Taxon. Bot. 34 (1): 50. 2010.
Cytisus cajan L., Sp. PI. 739. 1753.

Habit: Shrub, cultivated.

Specimens examined: 20.iii.2004, Rana farm Girijapuri, coll. S.D. Maliya 225924 (LWG).

Cajanus crassus (Prain ex King) Maesen, Agric. Univ. Wageningen Pap. 85 - 4: 105. 1985.

Atylosia crassa Prain ex King, J. Asiat. Soc. Bengal, Pt. 2, Nat. Hist. 66 (2): 45. 1897; Panigrahi et al., Bull. Bot. Surv. India 11 (1 \& 2): 94.1969.

Habit: Twining herb.

Specimens examined: 12.ii.1965, Nishangara range, O.P. Mishra 7947 (BSA).

Cajanus scarabaeoides (L.) Thouars, Dict. Sci. Nat. 6: 167. 1817; Maliya \& Datt, J. Econ. Taxon. Bot. 34 (1): 50. 2010.

Dolichos scarabaeoides L., Sp. PI. 726. 1753. Atylosia scarabaeoides (L.) Benth. in Miq., PI. Jungh. 242. 1852; Panigrahi et al., Bull. Bot. Surv. India 11 (1 \& 2): 95. 1969.

Habit: Twining herb.

Specimens examined: 26.v.1954, Motipur range, Hira Lal 13452 (LWG); 12.xii.1986, Motipur range, K.K. Khanna \& R. Saran 38663 (BSA); 09.xi.2002, road side, Katerniaghat range, coll. S.D. Maliya 224556 (LWG); 12.xi.2002, Nishangara range, coll. S.D. Maliya 224648 (LWG); 22.x.2010, Nishangara range, coll. S.D. Maliya 252619 (LWG).

Clitoria ternatea L., Sp. PI. 753. 1753; Baker in Hook. f., Fl. Brit. India 2: 208. 1879; Duthie, FI. Upp. Gang. Plain 1: 212. repr. ed. 1960; Saini, J. Econ. Taxon. Bot. 29 (3): 559. 2005.

Habit: Climber.

Specimens examined: Not observed. Record based on Saini (2005).

Crotalaria alata Buch. - Ham. ex D. Don, Prodr. Fl. Nepal. 241. 1825; Panigrahi et al., Bull. Bot. Surv. India 11 (1 \& 2): 95. 1969; Maliya \& Datt, J. Econ. Taxon. Bot. 34 (1): 50. 2010.

Habit: Shrub or undershrub.

Specimens examined: 20.xi.1964, Nshangara range, Katerniaghat Wildlife Sanctuary, Bahraich G. Panigrahi 6484 (BSA); 22.iv.2003, Nishangara range, coll. S.D. Maliya 224969 (LWG).

Crotalaria albida Heyne ex Roth., Nov. PI. Sp. 333. 1821; Panigrahi et al., Bull. Bot. Surv. India 11 (1 \& 2): 95. 1969; Maliya \& Datt, J. Econ. Taxon. Bot. 34 (1): 50. 2010. Habit: Perennial herb or undershrub. 
Specimens examined: 11.xi.2002, Murtiha range, coll. S.D. Maliya 224610 (LWG); 12.xi.2002, Nishangara range, coll. S.D. Maliya 224650 (LWG).

Crotalaria calycina Schrank, PI. Rar. Hort. Monac. t. 12. 1817; Panigrahi et al., Bull. Bot. Surv. India 11 (1 \& 2): 95. 1969; Maliya \& Datt, J. Econ. Taxon. Bot. 34 (1): 50. 2010.

Habit: Herb.

Specimens examined: 23.viii.2002, Nishangara range, coll. S.D. Maliya 223605 (LWG); 12.xi.2002, Nishangara range, coll. S.D. Maliya 224633 (LWG); 22.x.2010, Nishangara range, coll. S.D. Maliya 252620 (LWG).

Crotalaria medicaginea Lam., Encycl. 2: 201. 1786; Duthie, Fl. Upp. Gang. Plain 1: 189. repr. ed. 1960; Panigrahi et al., Bull. Bot. Surv. India 11 (1 \& 2): 95.1969. Habit: Perennial herb.

Specimens examined: Not observed. Record based on Panigrahi et al. (1969).

Crotalaria mysorensis Roth., Nov. PI. Sp. 338. 1821; Baker in Hook. f., Fl. Brit. India 2: 70. 1879; Saini, J. Econ. Taxon. Bot. 29 (3): 559. 2005.

Habit: Herb.

Specimens examined: Not observed. Record based on Saini (2005).

**Crotalaria pallida Ait., Hort. Kew. 3: 20. 1789; Saini, J. Econ. Taxon. Bot. 29 (3): 560. 2005.

C. mucronata Desv., J. Bot. Agric. 3: 76. 1814. C. striata DC., Prodr. 2: 131. 1825; Baker in Hook. f., Fl. Brit. India 2: 84. 1879.

Habit: Undershrub.

Specimens examined: Not observed. Record based on Saini (2005)

Crotalaria prostrata Rottl. ex Willd., Enum. PI. 2: 747. 1809; Panigrahi et al., Bull. Bot. Surv. India 11 (1 \& 2): 95. 1969; Maliya \& Datt, J. Econ. Taxon. Bot. 34 (1): 50. 2010.

Habit: Perennial herb.

Specimens examined: 12.xi.2002, Nishangara range, coll. S.D. Maliya 224649 (LWG); 24.iv.2003, Murtiha range, coll. S.D. Maliya 214823 (LWG).

Crotalaria sessiliflora L., Sp. PI. 1004. 1763; Panigrahi et al., Bull. Bot. Surv. India 11 (1 \& 2): 95. 1969; Maliya \& Datt, J. Econ. Taxon. Bot. 34 (1): 50. 2010.

Habit: Herb.

Specimens examined: 31.viii.2003, Fakirpur village, coll. S.D. Maliya 214882 (LWG).
Crotalaria spectabilis Roth.,Nov. PI. Sp. 341. 1821.

C. sericea Retz., Observ. Bot. 5: 26. 1788; Baker in Hook. f., Fl. Brit. India 2: 75. 1879; Duthie, Fl. Upp. Gang. Plain 1: 189. repr. ed. 1960; Panigrahi et al., Bull. Bot. Surv. India 11 (1 \& 2): 95. 1969

Habit: Herb or undershrub.

Specimens examined: 25.v.1954, Motipur range, Hira Lal 13403 (LWG); 12.iii.1964, Rampurwa, G. Panigrahi 2857 (BSA); 08.xii.1986, K.K. Khanna \& R. Saran 37634 (BSA); 28.ii.2011, rest house, Nishangara range, coll. S.D. Maliya 214911 (LWG).

Dalbergia latifolia Roxb., PI. Coromandel 2: 7, t. 113. 1798; Baker in Hook. f., FI. Brit. India 2: 231. 1879; Saini, J. Econ. Taxon. Bot. 29 (3): 560. 2005.

Amerimnon latifolium (Roxb.) Kuntze, Revis. Gen. PI. 1: 159.1891.

Habit: Tree.

Specimens examined: Not observed. Record based on Saini (2005).

Dalbergia sissoo Roxb. ex DC., Prodr. 2: 416. 1825; Baker in Hook. f., Fl. Brit. India 2: 231. 1879; Duthie, Fl. Upp. Gang. Plain 1: 243. repr. ed. 1960; Maliya \& Datt, J. Econ. Taxon. Bot. 34 (1): 50. 2010.

Amerimnon sissoo (Roxb. ex DC.) Kuntze, Revis. Gen. PI. 1: 159. 1891.

Habit: Tree.

Specimens examined: 17.iv.2003, Katerniaghat range, Katernniaghat wildlife sanctuary, Bahraich, coll. S.D. Maliya 224906 (LWG), 20.iv.2003, Murtiha range, Katernniaghat wildlife sanctuary, Bahraich, coll. S.D. Maliya 224951 (LWG); 21.iv.2003, Nishangara range, Katernniaghat wildlife sanctuary, Bahraich, coll. S.D. Maliya 224962 (LWG); 19.vii.2003, Kuthiyaghat forest, Katernniaghat wildlife sanctuary, Bahraich, coll. S.D. Maliya 225968 (LWG); 16.iii.2008, Murtiha range, Katernniaghat wildlife sanctuary, Bahraich, coll. S.D. Maliya 218514 (LWG).

Dendrolobium triangulare (Retz.) Schindl., Repert. Spec. Nov. Regni Veg. 20: 279. 1924.

Hedysarum triangulare Retz., Observ. Bot. 3: 40. 1783. Desmodium cephalotes W. \& A., Prodr. 224. 1834; Baker in Hook. f., Fl. Brit. India 2: 161. 1879. D. triangulare (Retz.) Merr., J. Arnold Arbor. 23: 170. 1942; Panigrahi et al., Bull. Bot. Surv. India 11 (1 \& 2): 95. 1969; Maliya \& Datt, J. Econ. Taxon. Bot. 34 (1): 51. 2010.

Habit: Shrub or undershrub.

Specimens examined: 21.iv.1964, Nishangara range, G. Panigrahi \& O.P. Mishra 6503 (BSA); 12.ii.1965, 
Nishangara range, O.P. Mishra 7953 (BSA); 08.xii.1986, Nishangara range, K.K. Khanna \& R. Saran 37603 (BSA); 11.xi.2002, Murtiha range, Katernniaghat wildlife sanctuary, Bahraich, coll. S.D. Maliya 224615 (LWG).

Desmodium gangeticum (L.) DC., Prodr. 2: 327. 1825; Baker in Hook. f., Fl. Brit. India 2: 168. 1879; Duthie, Fl. Upp. Gang. Plain 1: 264. repr. ed. 1960; Panigrahi et al., Bull. Bot. Surv. India 11 (1 \& 2): 95. 1969; Maliya \& Datt, J. Econ. Taxon. Bot. 34 (1): 50. 2010.

Hedysarum gangeticum L., Sp. PI. 746. 1753. Desmodium gangeticum var. maculatum (L.) Baker in Hook. f., Fl. Brit. India 2(4): 168. 1876.

Habit: Shrub or undershrub.

Specimens examined: 24.xi.1964, Nishangara range, G. Panigrahi \& O.P. Mishra 6606A (BSA); 15.iv.1967, S. L. Kapoor \& party 70046 (LWG); 25.viii.2002, Murtiha range, coll. S.D. Maliya 223722 (LWG); 11.xi.2002, Murtiha range, coll. S.D. Maliya 224614 (LWG); 12.xi.2002, Nishangara range, coll. S.D. Maliya 224646 (LWG); 17.iv.2003, Katerniaghat range, coll. S.D. Maliya 224919 (LWG); 22.iv.2003, Nishangara range, coll. S.D. Maliya 225000 (LWG).

Desmodium heterocarpon (L.) DC., Prodr. 2: 337. 1825; Panigrahi et al., Bull. Bot. Surv. India 11 (1 \& 2): 95 1969; Maliya \& Datt, J. Econ. Taxon. Bot. 34 (1): 51.2010.

Hedysarum hetercarpon L., Sp. PI. 747. 1753. Desmodium polycarpon DC., Prodr. 2: 334. 1825; Baker in Hook. f., Fl. Brit. India 2: 171. 1879.

Habit: Undershrub.

Specimens examined: 21.ii.1965, Nishangara range, O.P. Mishra 7950 (BSA); 11.xi.2002, Murtiha range, Katernniaghat wildlife sanctuary, Bahraich, coll. S.D. Maliya 223608 (LWG).

**Desmodium triflorum (L.) DC., Prodr. 2: 334. 1825 ; Baker in Hook. f., Fl. Brit. India 2: 173. 1879; Duthie, Fl. Upp. Gang. Plain 1: 263. repr. ed. 1960; Maliya \& Datt, J. Econ. Taxon. Bot. 34 (1): 51. 2010.

Hedysarum triflorum L., Sp. PI. 749. 1753.

Habit: Perennial herb.

Specimens examined: 23.viii.2002, Nishangara range, Katernniaghat wildlife sanctuary, Bahraich, coll. S.D. Maliya 223632 (LWG); 09.xi.2002, Katerniaghat range, Katernniaghat wildlife sanctuary, Bahraich, coll. S.D. Maliya 224530 (LWG); 09.xi.2002, Katerniaghat range, Katernniaghat wildlife sanctuary, Bahraich, coll. S.D. Maliya 224541 (LWG);
Desmodium velutinum (Willd.) DC., Prodr. 2: 328. 1825; Knaap-vam Meeuwen, Reinwardtia (3): 264. 1962; Panigrahi et al., Bull. Bot. Surv. India 11 (1 \& 2): 95. 1969; Maliya \& Datt, J. Econ. Taxon. Bot. 34 (1): 51. 2010.

Hedysarum velutinum Willd., Sp. PI. 3: 117. 1803. Desmodium latifolium (Roxb.) DC., Prodr. 2:328. 1825; Baker in Hook. f., FI. Brit. India 2: 168. 1879. Hedysarum latifolium Roxb., Fl. Ind. 3: 350. 1832.

Habit: Undershrub.

Specimens examined: 24.viii.2002, Nishangara range, Katernniaghat wildlife sanctuary, Bahraich, coll. S.D. Maliya 223675 (LWG).

Dolichos trilobus L., Sp. PI. 726. 1753; Panigrahi et al., Bull. Bot. Surv. India 11 (1 \& 2): 95.1969.

D. falcatus Klein ex Willd., Sp. PI. 1047. 1802.

Habit: Climber.

Specimens examined: 21.iv.1964, Murtiha gate, G. Panigrahi 2890 (BSA) (Image 33).

Erythrina arborescens Roxb., Pl. Coromandel 3: 14, t. 219. 1819; Maliya \& Datt, J. Econ. Taxon. Bot. 34 (1): 51. 2010.

Habit: Tree.

Specimens examined: 22.iii.2004, Nishangara range, coll. S.D. Maliya 225936 (LWG).

Flemingia chappar Buch.-Ham. ex Benth. in Miq., PI. Jungh. 244. 1852.

Moghania chappar (Buch.-Ham. ex Benth.) Kuntze., Rev. Gen. PI. 1: 199. 1891; Panigrahi et al., Bull. Bot. Surv. India 11 (1 \& 2): 96. 1969.

Habit: Shrub.

Specimens examined: 21.iv.1964, Motipur range, G. Panigrahi 2845 (BSA); 22.xi.1964, Motipur on the way to Orla, G. Panigrahi \& O.P. Mishra 6532 (BSA); 07.xii.1986, Motipur range, K.K. Khanna \& R. Saran 38561 (BSA).

Flemingia lineate (L.) Roxb. ex Ait., Hort. Kew. 4: 350. 1812.

Hedysarum lineatum L., Syst. Nat. ed. 10. 2: 1170. 1759. Moghania lineata (L.) Kuntze., Revis. Gen. PI. 1: 199. 1891; Panigrahi et al., Bull. Bot. Surv. India 11 (1 \& 2): 96.1969.

Habit: Shrub.

Specimens examined: 14.ii.1965, Uraital range, O.P. Mishra 7992 (BSA); 09.xii.1986, K.K. Khanna \& R. Saran 37679 (BSA).

Flemingia macrophylla (Willd.) Kuntze ex Merr., Phillip. J. Sci. 5(2): 130. 1910. 
Crotalaria macrophylla Willd., Sp. PI. 3(2): 982. 1802. Flemingia semialata Roxb. ex Ait. f., Hort. Kew. 4: 349. 1812; Maliya \& Datt, J. Econ. Taxon. Bot. 34 (1): 51.2010. Moghania macrophylla (Willd.) Kuntze., Revis. Gen. PI. 1:199. 1891; Panigrahi et al., Bull. Bot. Surv. India 11 (1 \& 2): 96. 1969. M. semialata (Roxb. ex Ait. f.) Mukherjee, Bull. Bot. Soc. Bengal 6: 16. 1952.

Habit: Shrub.

Specimens examined: 21.xi.1964, Nishangara range, G. Panigrahi 6504 (BSA); 08.xii.1986, Nishangara range, K.K. Khanna \& R. Saran 37605 (BSA); 07.xii.1986, Motipur range, K.K. Khanna \& R. Saran 38573 (BSA).

Flemingia paniculata Wall. ex Benth., PI. Jungh. 2: 245. 1852

Moghania paniculata (Wall.) H. L. Li., Amer. J. Bot. 31(4): 227. 1944; Panigrahi et al., Bull. Bot. Surv. India 11 (1 \& 2): 96.1969.

Habit: Shrub.

Specimens examined: 15.iii.1964, Rampurwa, G. Panigrahi 2904 (BSA) (Image 34).

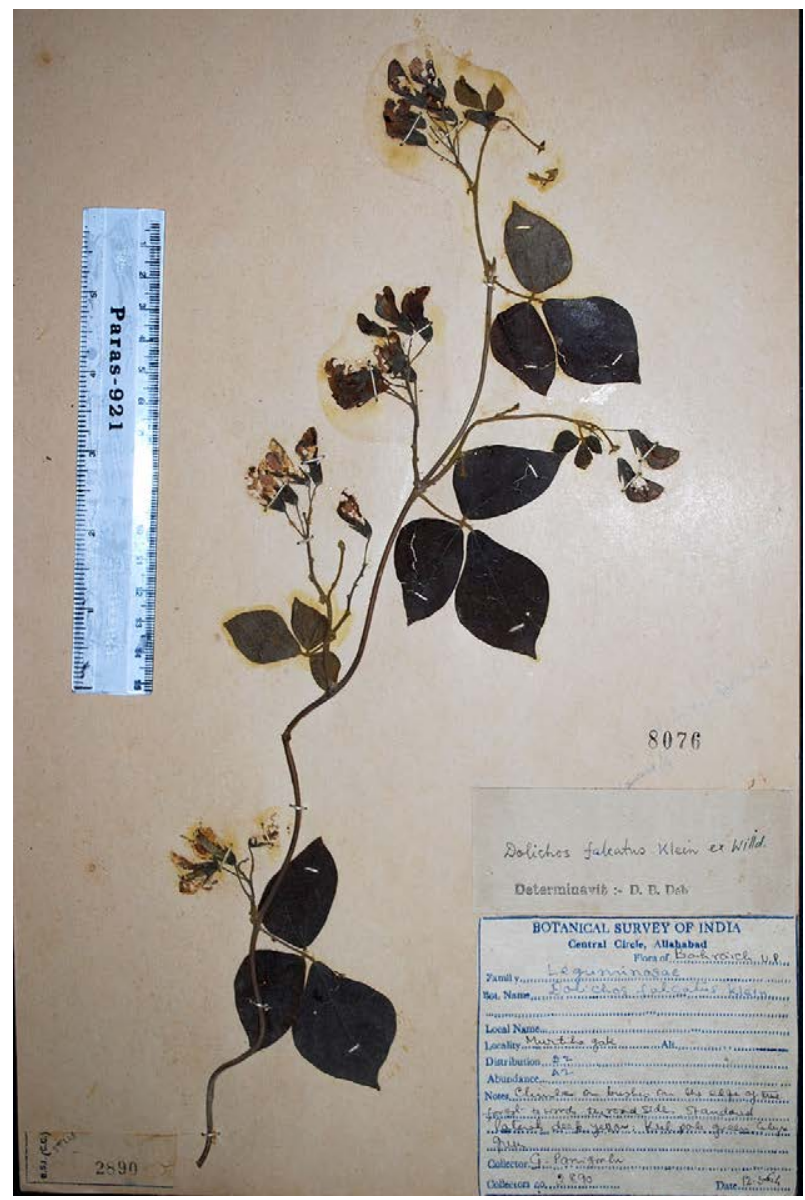

Image 33. Dolichos trilobus L.
Flemingia prostrata Roxb., Fl. Ind. 3: 338. 1832.

Moghania prostrata (Roxb.) Mukerjee, Bull. Bot. Soc. Bengal 6(1): 19. 1953; Panigrahi et al., Bull. Bot. Surv. India 11 (1 \& 2): 96.1969.

Habit: Undershrub.

Specimens examined: 12.iii.1964, Murtiha gate, G. Panigrahi 2888 (BSA); 20.xi.1964, Nishangara range, G. Panigrahi \& O.P. Mishra 6458 (BSA); 12.ii.1965, Nishangara range, O.P. Mishra 7946 (BSA); 13.ii.1965, Nishangara range, O.P. Mishra 7978 (BSA).

Flemingia strobilifera (L.) Ait., Hort. Kew 4: 350. 1812; Baker in Hook. f., Fl. Brit. India 2: 227. 1879; Saini, J. Econ. Taxon. Bot. 29 (3): 561. 2005; Maliya \& Datt, J. Econ. Taxon. Bot. 34 (1): 51. 2010.

Hedysarum strobiliferum L., Sp. PI. 746. 1753. Moghania strobilifera (L.) St. Hill ex Kuntze, Desv. J. Bot. Appl. 1: 62. 1813; Panigrahi et al., Bull. Bot. Surv. India 11 (1 \& 2): 96.1969.

Habit: Shrub.

Specimens examined: 14.ii.1965, Uraital range, O.P. Mishra 7993 (BSA); 07.xii.1986, Motipur range, K.K. Khanna \& R. Saran 38563 (BSA); 26.xi.1987, Nishangara range, K.K. Singh \& party 6911 (BSA); 10.xi.2002, Murtiha range, coll. S.D. Maliya 224587 (LWG); 12.xi.2002, Nishangara range, coll. S.D. Maliya 224634 (LWG); 18.03.2004, Kuthiyaghat forest, Katerniaghat Wildlife Sanctuary, Baharaich, coll. S.D. Maliya 225909 (LWG).

Flemingia strobilifera (L.) W. T. Aiton var. nudiflora Haines, Bot. Bihar \& Orissa 3: 280. 1922.

Moghania strobilifera (L.) St. Hill ex Kuntze. var. nudiflora (Haines) Mukerjee, Bull. Bot. Soc. Bengal 6: 11. 1952; Panigrahi et al., Bull. Bot. Surv. India 11 (1 \& 2): 96. 1969.

Habit: Shrub.

Specimens examined: 12.ii.1965, Nishangara range, O.P. Mishra 7945 (BSA).

Indigofera astrgalina DC., Prodr. 2: 228. 1825; Saini, J. Econ. Taxon. Bot. 29 (3): 562. 2005.

Habit: Herb.

Specimens examined: Not observed. Record based on Saini (2005).

Indigofera cassoides Rottl. ex DC., Prodr. 2: 225. 1825; Panigrahi et al., Bull. Bot. Surv. India 11 (1 \& 2): 95.1969.

Habit: Shrub.

Specimens examined: 12.iii.1964, Murtiha gate, G. Panigrahi 2887 (BSA); 10.ii.1965, Nishangara range, O.P. Mishra 7903 (BSA). 


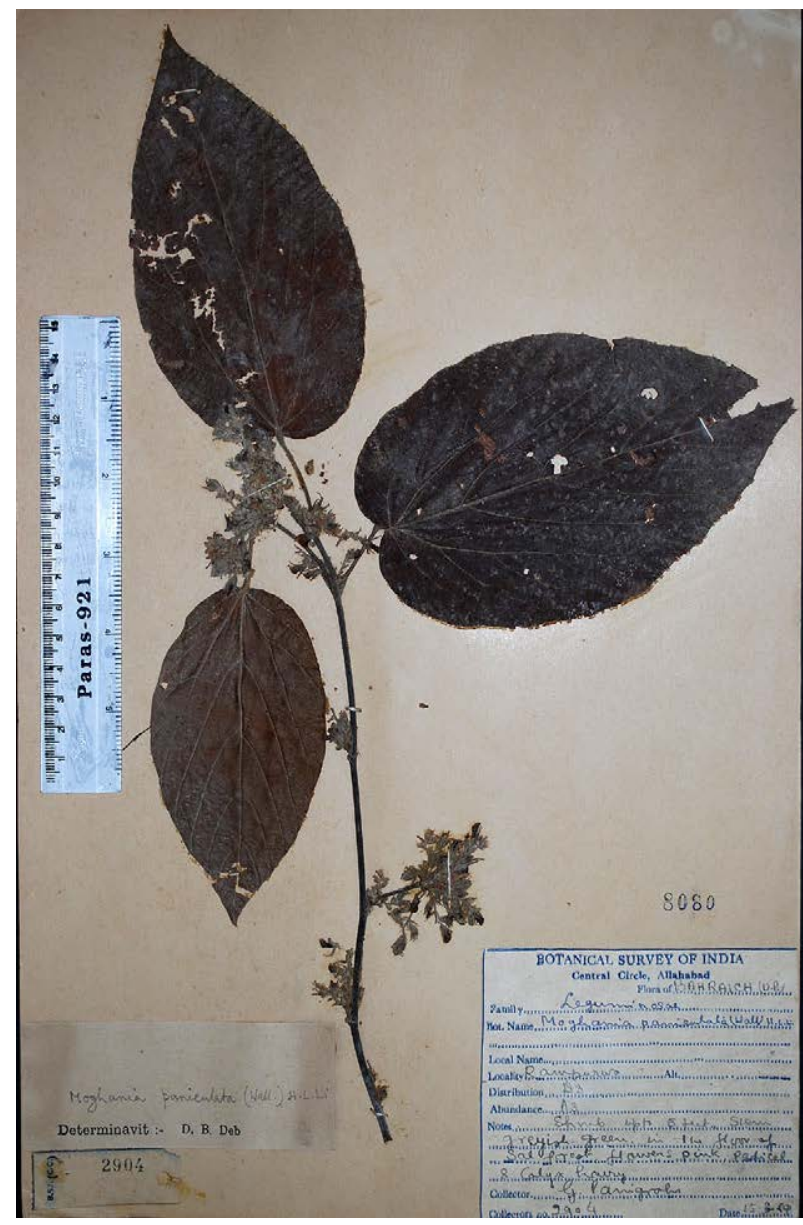

Image 34. Flemingia paniculata Wall. ex Benth.

Indigofera glandulosa Wendl., Bot. Beob. 55. 1798; Maliya \& Datt, J. Econ. Taxon. Bot. 34 (1): 51. 2010.

Habit: Perennial herb.

Specimens examined: 17.iv.2003, road side, Katerniaghat range, coll. S.D. Maliya 224921 (LWG).

Indigofera linifolia (L. f.) Retz., Observ. Bot. 4: 29. 1786; Baker in Hook. f., Fl. Brit. India 2: 92. 1879; Duthie, Fl. Upp. Gang. Plain 1: 229. repr. ed. 1960; Maliya \& Datt, J. Econ. Taxon. Bot. 34 (1): 51. 2010.

Hedysarum linifolium L. f., Suppl. PI. 331. 1782.

Habit: Perennial herb.

Specimens examined: 09.xi.2002, road side, Katerniaghat range, coll. S.D. Maliya 224540 (LWG).

Indigofera thothathrii Sanjappa, J. Jap. Bot. 50: 272. 1984 \& in Hajra et al., Fasc. Fl. India 21: 133, f. 50a-e. 1995. Habit: Herb.

Specimens examined: Not observed. Record based on Sanjappa (1984).
Indigofera trifoliate L., Cent. pl. II: 29. 1756 (Amoen. acad. 4:327. 1759).

Habit: Herb.

Specimens examined: 20.iii.2008, Lahaura village, coll. S.D. Maliya 218576 (LWG).

* Lablab purpureus (L.) Sweet, Hort. Brit. ed. 1. 481. 1826; Saini, J. Econ. Taxon. Bot. 29 (3): 562. 2005; Maliya \& Datt, J. Econ. Taxon. Bot. 34 (1): 51. 2010.

Dolichos purpureus L., Sp. PI. 1021. 1763. D. lablab L., Sp. PI. 725. 1753; Panigrahi et al., Bull. Bot. Surv. India 11 (1 \& 2): 95. 1969.

Habit: Twining herb, cultivated.

Specimens examined: 24.xi.1987, K.K. Singh \& party 6173 (LWG); 20.iii.2004, Rana farm, Girijapuri, coll. S.D. Maliya 225931 (LWG).

**Lathyrus aphaca L., Sp. Pl. 729. 1753; Baker in Hook. f., Fl. Brit. India 2: 179. 1876; Duthie, Fl. Upp. Gang. Plain 1: 239. repr. ed. 1960; Saini, J. Econ. Taxon. Bot. 29 (3): 562. 2005.

Habit: Annul herb.

Specimens examined: Not observed. Record based on Saini (2005).

** Lathyrus sativus L., Sp. PI. 730. 1753; Baker in Hook. f., Fl. Brit. India 2: 179. 1879; Duthie, Fl. Upp. Gang. Plain 1: 240. repr. ed. 1960; Maliya \& Datt, J. Econ. Taxon. Bot. 34 (1): 51.2010.

Habit: Annual herb.

Specimens examined: 20.iii.2004, Girijapuri forest, coll. S.D. Maliya 225927 (LWG).

Lathyrus sphaericus Retz., Observ. Bot. 3: 39. 1783; Panigrahi et al., Bull. Bot. Surv. India 11 (1 \& 2): 95. 1969.

L. coccineus All., Fl. Pedem. 1: 330. 1785.

Habit: Annual herb.

Specimens examined: 09.ii.1965, Jairampurwa, O.P. Mishra 7763 (BSA).

*Lens culinaris Medik., Vorles Churpfalz. Phys.-Okon. Ges. 2: 361. 1787; Maliya \& Datt, J. Econ. Taxon. Bot. 34 (1): 51.2010.

Ervum lens L., Sp. PI. 738. 1753.

Habit: Herb, cultivated.

Specimens examined: 20.iii.2004, Girijapuri forest, coll. S.D. Maliya 225922 (LWG).

Medicago lupulina L., Sp. PI. 779. 1753; Baker in Hook. f., Fl. Brit. India 2: 90. 1879; Duthie, Fl. Upp. Gang. Plain 1: 194. repr. ed. 1960; Panigrahi et al., Bull. Bot. Surv. India 
11 (1 \& 2): 95.1969.

Trifolium dubium sensu Panigrahi et al., Bull. Bot. Surv. India 11 (1 \& 2): 96. 1969.

Habit: Trailing biennial herb.

Specimens examined: 11.iii.1964, Sujauli, G. Panigrahi 2798 (BSA); 16.ii.1965, O.P. Mishra 8019 (BSA).

**Medicago polymorpha L., Sp. PI. 779. 1753; Steenis, Blumea 12: 15. 1962; Saini, J. Econ. Taxon. Bota. 29 (3): 563. 2005.

M. denticulata Willd., Sp. PI. 3 (2): 1414. 1802; Baker in Hook. f., Fl. Brit. India 2: 90. 1879; Duthie, Fl. Upp. Gang. Plain 1: 194. repr. ed. 1960.

Habit: Annual herb.

Specimens examined: Not observed. Record based on Saini (2005)

Melilotus indicus (L.) All., Fl. Pedem. 1: 308. 1785; Saini, J. Econ. Taxon. Bot. 29 (3): 563. 2005; Maliya \& Datt, J. Econ. Taxon. Bot. 34 (1): 51. 2010.

Trifolium indicum L., Sp. PI. 765. 1753 "Trifolium M. indica". Melilotus parviflorus Desf., FI. Atlant. 2: 192. 1799 "parviflora"; Baker in Hook. f., Fl. Brit. India 2: 89. 1879.

Habit: Herb.

Specimens examined: 09.ii.1965, Bhagara nala, Motipur, O.P. Mishra 7782 (BSA); 21.xii.2003, Katerniaghat range, coll. S.D. Maliya 225229 (LWG); 20.iii.2004, Girijapuri forest, coll. S.D. Maliya 225921 (LWG).

Millettia extensa (Benth.) Benth. ex Baker in Hook. f., FI. Brit. India 2(4): 109. 1879; Ohashi in Hara et al., Enum. FI. PI. Nepal 2: 125. 1979; Maliya \& Datt, J. Econ. Taxon. Bot. 34 (1): 51.2010.

Otosema extensa Benth in Miq., PI. Jungh. 249. 1825. Millettia auriculata Baker ex Brandis, For. FI. N. W. Ind. 138. 1874; Baker in Hook. f., Fl. Brit. India 2: 108. 1879; Panigrahi et al., Bull. Bot. Surv. India 11 (1 \& 2): 96.1969.

Habit: Climber.

Specimens examined: 12.iii.1964, Motipur range, G. Panigrahi 2856 (BSA); 08.xii.1986, Bichhiya, K.K. Khanna \& R. Saran 37637 (BSA); 21.v.1990, Bichhiya, K.K. Khanna 39923 (BSA); 29.iv.2003, road side, Murtiha range, coll. S.D. Maliya 224940 (LWG); 30.viii.2003, road side, Murtiha range, coll. S.D. Maliya 214870 (LWG).

Mucuna pruriens (L.) DC., Prodr. 2: 405. 1825; Baker in Hook. f., Fl. Brit. India 2: 187. 1879; Duthie, Fl. Upp. Gang. Plain 1: 218. repr. ed. 1960; Saini, J. Econ. Taxon. Bot. 29 (3): 563. 2005.

Dolichos pruriens L., Diss. Herb. Amb. 23. 1754. Mucuna prurita Hook., Bot. Misc. 2: 348. 1830; Panigrahi et al., Bull. Bot. Surv. India 11 (1 \& 2): 96. 1969.

Habit: Climber.

Specimens examined: Not observed. Record based on Panigrahi et al. (1969) and Saini (2005).

Ougeinia oojeinensis (Roxb.) Hochr., Annuaire Conserv. Jard. Bot. Geneve 13-14: 51. 1909.

Dalbergia oojeinensis Roxb., Fl. Ind. 3: 220. 1832. Desmodium oojeinense (Roxb.) Ohashi, Ginkgoana 1: 117. 1973.

Habit: Tree.

Specimens examined: 04.iii.2011, near guest house, Nishangarha range, Katerniaghat Wildlife Sanctuary, A. Kumar \& O. Bajpai 252853 (LWG).

Phyllodium pulchellum (L.) Desv., J. Bot. Agric. 1: 124, t. 5. 1813 "pulchrum".

Hedysarum pulchellum L., Sp. PI. 747. 1753. Desmodium pulchellum (L.) Benth., Fl. Hong Kong 86. 1861; Baker in Hook. f., Fl. Brit. India 2: 162. 1879; Panigrahi et al., Bull. Bot. Surv. India 11 (1 \& 2): 95. 1969; Maliya \& Datt, J. Econ. Taxon. Bot. 34 (1): 51. 2010.

Habit: Shrub.

Specimens examined: 14.iii.1964, Rmpurwa, near Nepal Border, G. Panigrahi 2896 (BSA); 07.xii.1986, Motipur range, K.K. Khanna \& R. Saran 38562 (BSA); 23.viii.2002, Nishangara range, Katernniaghat wildlife sanctuary, Bahraich, coll. S.D. Maliya 223610 (LWG).

Pongamia pinnata (L.) Pierr., Fl. Cochinch. Sub. t. 385. 1899; Thothathri, Bull Bot. Surv. India 3: 417. 1961.

Cytisus pinnatus L., Sp. Pl. 741. 1753. Pongamia glabra Went., Jard Malm. 28. 1803; Baker in Hook. f., Fl. Brit. India 2: 240. 1879. Millettia pinnata (L.) Panigrahi in Panigrahi \& Murti, Fl. Bilaspur Distr. 1: 210. 1989.

Habit: Tree.

Specimens examined: 20.iv.2010, Salarpur, L.B. Chaudhary, A. Kumar \& O. Bajpai 252213 (LWG) (Image 35).

Rhynchosia minima (L.) DC., Prodr. 2: 385. 1825; Baker in Hook. f., Fl. Brit. India 2: 223. 1879; Duthie, Fl. Upp. Gang. Plain 1: 204. repr. ed. 1960; Panigrahi et al., Bull. Bot. Surv. India 11 (1 \& 2): 96. 1969; Saini, J. Econ. Taxon. Bot. 29 (3): 564. 2005.

Dolichos minimus L., Sp. PI. 726. 1753.

Habit: Twining herb.

Specimens examined: 21.xi.1964, Nishangara range, G. Panigrahi \& O.P. Mishra 6507 (BSA). 


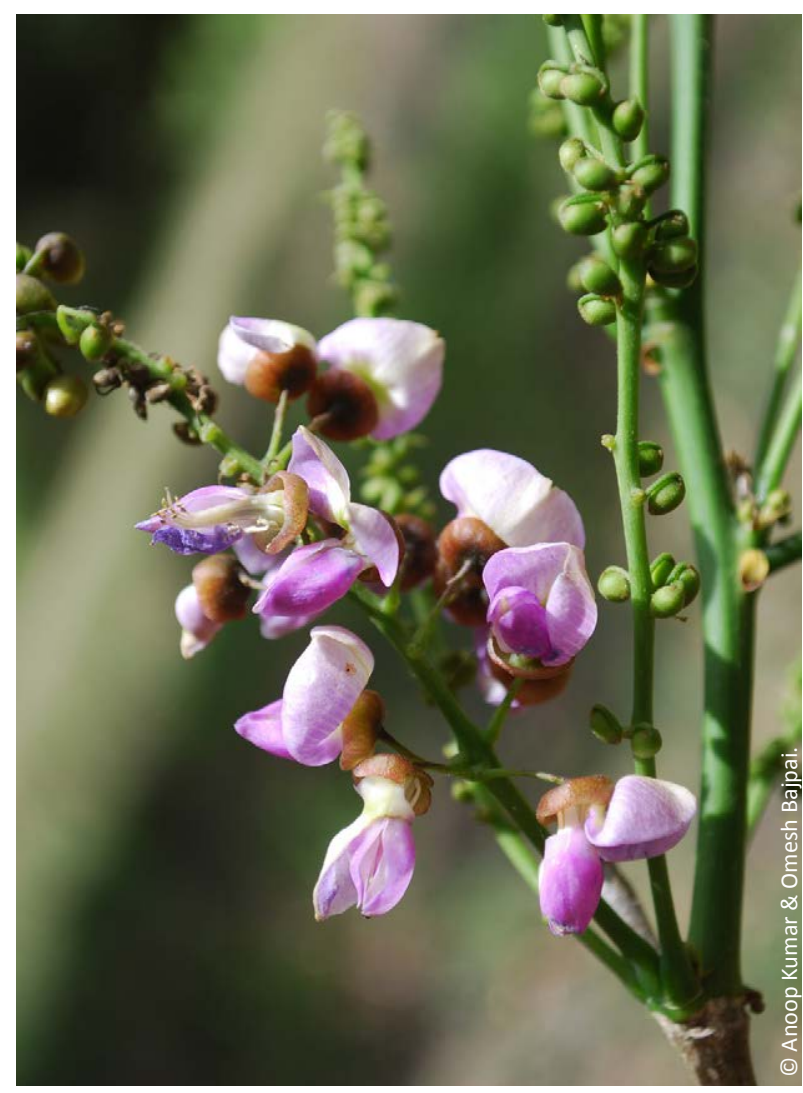

Image 35. Pongamia pinnata (L.) Pierr.

Rhynchosia rothii Benth. ex Aitch., Cat. PI. Punjab. 50. 1869.

R. sericea Span., Linnaea 15: 195. 1841; Panigrahi et al., Bull. Bot. Surv. India 11 (1 \& 2): 96. 1969.

Habit: Climber.

Specimens examined: 21.xi.1964, Nishangara range, G. Panigrahi \& O.P. Mishra 6509 (BSA).

Sesbania bispinosa (Jacq.) W. Wight, U.S.D.A. Bur. PI. Industr. Bull. 137: 15. 1909; Maliya \& Datt, J. Econ. Taxon. Bot. 34 (1): 51.2010.

Aeschynomene bispinosa Jacq., Icon. PI. Rar. 3: 13, t. 564. 1793. Coronilla aculeate Willd., Sp. PI. 3(2): 1147. 1802. Sesbania aculeata (Willd.) Pers., Syn. PI. 2(2): 316. 1807; Baker in Hook. f., Fl. Brit. India 2: 114. 1879.

Habit: Shrub.

Specimens examined: 31.viii.2003, road side, Fakirpur village, coll. S.D. Maliya 214871 (LWG).

Tephrosia purpurea (L.) Pers., Syn. PI. 2(2):329. 1807; Maliya, J. Econ. Taxon. Bot. 36 (2): 421. 2012.

Cracca purpurea L., Sp. PI. 2: 752. 1753.

Habit: Herb.

Specimens examined: 25.v.1954, Motipur range, Hira
Lal 13413 (LWG); 05.xi.2008, road side, coll. S.D. Maliya 226632 (LWG).

Teramnus labialis (L. f.) Spreng., Syst. 3: 235. 1826; Baker in Hook. f., Fl. Brit. India 2: 184. 1879; Duthie, Fl. Upp. Gang. Plain 1: 214. repr. ed. 1960; Saini, J. Econ. Taxon. Bot. 29 (3): 565. 2005.

Glycine labialis L. f., Suppl. 325. 1781.

Habit: Twining herb.

Specimens examined: Not observed. Record based on Saini (2005).

**Trigonella foenum-graecum L., Sp. PI. 777. 1753; Baker in Hook. f., Fl. Brit. India 2: 87. 1879; Duthie, Fl. Upp. Gang. Plain 1: 192. repr. ed. 1960; Panigrahi et al., Bull. Bot. Surv. India 11 (1 \& 2): 96. 1969.

Habit: Annual herb.

Specimens examined: 12.iii.1964, Murtihagate, G. Panigrahi 2865 (BSA).

Uraria lagopus var. lagopus DC., Ann. Sci. Nat. 4: 100. Jan. 1825 \& Prod. 2: 324. 1825; Sanjappa, Legumes of India. 267. 1992.

Uraria alopecuroides (Roxb.) Wight, Ic. PI. Ind.Or.t. 290. 1840. Doodia alopeuroides Roxb., Fl. Ind. 3: 368. 1832.

Habit: Undershrub.

Specimens examined: 11.xi.2002, Murtiha range, coll. S.D. Maliya 224618 (LWG).

Uraria lagopus DC. var. neglecta (Prain) Ohashi, Fl. E. Himalaya 70. 1975; Maliya \& Datt, J. Econ. Taxon. Bot. 34 (1): 51. 2010.

U. neglecta Prain, J. Asiat. Soc. Bengal, Pt. 2, Nat. Hist. 66: 382. 1897.

Habit: Undershrub.

Specimens examined: 09.xi.2002, road side, Katerniaghat range, coll. S.D. Maliya 224535 (LWG); 11.xi.2002, Murtiha range, coll. S.D. Maliya 224609 (LWG); 05.xi.2008, road side, Katerniaghat range, coll. S.D. Maliya 226638 (LWG).

Uraria picta (Jacq.) Desv. ex DC., Prodr. 2: 324. 1825; Baker in Hook. f., Fl. Brit. India 2: 155. 1879; Saini, J. Econ. Taxon. Bot. 29 (3): 566. 2005; Maliya \& Datt, J. Econ. Taxon. Bot. 34 (1): 51.2010.

Hedyserum pictum Jacq., Collectanea 2: 262. 1788.

Habit: Shrub.

Specimens examined: 11.xi.2002, Murtiha range, coll. S.D. Maliya 224611 (LWG); 11.xi.2002, Murtiha range, coll. S.D. Maliya 224616 (LWG); 12.xi.2002, Nishangara 
range, coll. S.D. Maliya 224644 (LWG).

**Vicia sativa L., Sp. PI. 736. 1753; Baker in Hook. f., FI. Brit. India 2: 178. 1879; Panigrahi et al., Bull. Bot. Surv. India 11 (1 \& 2): 96. 1969.

V. leucosperma Moench., Methodus 148. 1794.

Habit: Herb.

Specimens examined: 14.ii.1965, Urai tal, O.P. Mishra 7985 (BSA).

*Vigna mungo (L.) Hepper in Kew Bull. 11: 128. 1956; Verdc., Kew Bull. 24. 558. 1970; Saini, J. Econ. Taxon. Bot. 29 (3): 566. 2005.

Phaseolus mungo L., Mant. PI. 1: 101. 1767; Baker in Hook. f. Fl. Brit. India, 2: 203. 1879; Duthie, Fl. Upp. Gang. Plain 1: 208. repr. ed. 1960.

Habit: Twining herb, cultivated.

Specimens examined: Not observed. Record based on Saini (2005).

Vigna trilobata (L.) Verdc., Taxon 17: 172. 1968; Saini, J. Econ. Taxon. Bot. 29 (3): 566. 2005.

Dolichos trilobatus L., Mant. PI. 101. 1767.

Habit: Twinning herb.

Specimens examined: Not observed. Record based on Saini (2005)

*Vigna unguiculata (L.) Walp. subsp. cylindrica (L.) Van Zseltine in Hedrick, Veg. New York 1 (2): 11. 1931; Verdc., Kew Bull. 24: 554. 1970; Saini, J. Econ. Taxon. Bot. 29 (3): 567. 2005.

Phaseolus cylindricus L., Herb. Amb. 23. 1751 \& Amoen. Acad. 4: 132. 1759.

Habit: Twining herb, cultivated.

Specimens examined: Not observed. Record based on Saini (2005).

Zornia gibbosa Span., Linnaea 15. 192. 1841; Saini, J. Econ. Taxon. Bota. 29 (3): 567. 2005.

Habit: Herb.

Specimens examined: Not observed. Record based on Saini (2005)

\section{GENTIANACEAE}

Canscora diffusa (Vahl) R. Br. ex Roem. \& Schult., Syst. Veg. 3: 301. 1818; Clarke in Hook. f. in Fl. Brit. India 4: 103. 1883; Duthie, Fl. Upp. Gang. Plain 1: 522. repr. ed. 1960; Saini, J. Econ. Taxon. Bot. 29 (3): 616. 2005.

Gentiana diffusa Vahl, Symb. Bot. 3: 47. 1794.

Habit: Annual herb.

Specimens examined: 26.v.1954, Motipur range, Hira
Lal 13443 (LWG) (Image 36).

Exacum tetragonum Roxb., Fl. Ind. 1: 398. 1832; Panigrahi et al., Bull. Bot. Surv. India 11 (1 \& 2): 104. 1969. Habit: Annual herb.

Specimens examined: 20.xi.1964, Nishangara range, G. Panigrahi \& O.P. Mishra 6473 (BSA).

Hoppea dichotoma Willd., Ges. Naturf. Freunde Berlin Neue Schriften 3: 435. 1801; Clarke in Hook. f., Fl. Brit. India 4: 100. 1883; Duthie, Fl. Upp. Gang. Plain 1: 522. repr. ed. 1960; Panigrahi et al., Bull. Bot. Surv. India 11 (1 \& 2): 104. 1969.

Habit: Annual herb.

Specimens examined: 16.ii.1965, Katerniaghat range, O.P. Mishra 8020 (BSA).

\section{HYDROLEACEAE}

Hydrolea zeylanica (L.) Vahl, Symb. Bot. 2: 46. 1791; Hook. f. in Hook. f., Fl. Brit. India 4: 133; Duthie, Fl. Upp. Gang. Plain 1: 526. repr. ed. 1960; Backer in Fl. Males. Ser. 1, 4 (3): 207. 1957; Panigrahi et al., Bull. Bot. Surv. India 11 (1 \& 2): 108. 1969; Maliya \& Datt, J. Econ. Taxon. Bot. 34 (1): 57. 2010.

Nama zeylanica L., Sp. PI. 226. 1753.

Habit: Annual herb.

Specimens examined: 26.x.2004, Nepal border, Murtiha range, coll. S.D. Maliya 227124 (LWG).

\section{LAMIACEAE}

Anisomeles indica (L.) Kuntze, Revis. Gen. PI. 2: 512. 1891; Raizada, Suppl. FI. Upp. Gang. Plain 215. 1976; Panigrahi et al., Bull. Bot. Surv. India 11 (1 \& 2): 108. 1969; Maliya \& Datt, J. Econ. Taxon. Bot. 34 (1): 60. 2010.

Nepeta indica L., Sp. PI. 571. 1753. Anisomeles ovata R. Br., Hort. Kew ed. 2. 3: 364. 1811; Hook. f. in Hook. f., Fl. Brit. India 4: 672. 1885; Duthie, Fl. Upp. Gang. Plain 2: 109. repr. ed. 1960.

Habit Herb or undershrub.

Specimens examined: 26.v.1954, Motipur range, Hira Lal 13432 (LWG); 09.xi.2002, Katerniaghat range, coll. S.D. Maliya 224547 (LWG); 10.xi.2002, Murtiha range, coll. S.D. Maliya 224580 (LWG); 12.xi.2002, Nishangara range, coll. S.D. Maliya 224654 (LWG); 22.x.2004, Kuthiyaghat forest, Katernighat wildlife sanctuary, Bahraich, coll. S.D. Maliya 225993 (LWG).

Callicarpa macrophylla Vahl, Symb. Bot. 3: 13, t. 53. 1794; Clarke in Hook. f., Fl. Brit. India 4: 568. 1885; Duthie, FI. Upp. Gang. Plain 2: 86. repr. ed. 1960; Maliya \& Datt, J. Econ. Taxon. Bot. 34 (1): 60. 2010. 


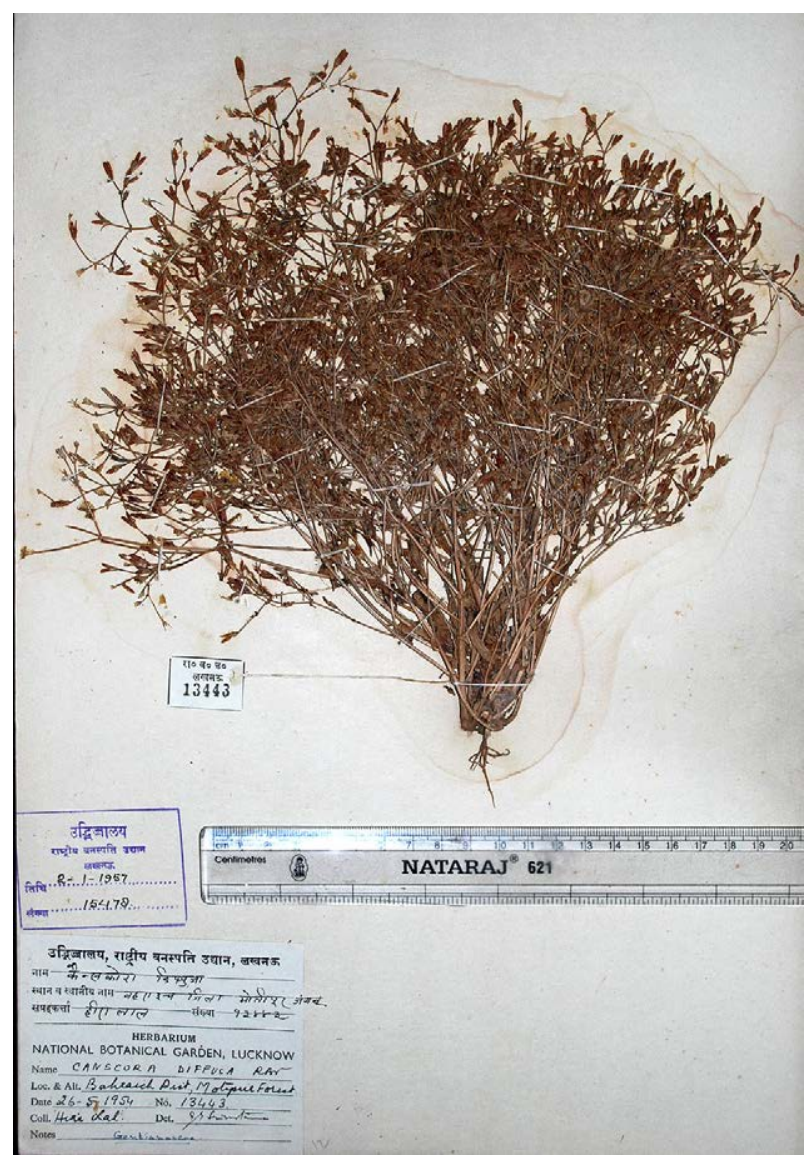

Image 36. Canscora diffusa (Vahl) R. Br. ex Roem. \& Schult.

Habit: Shrub.

Specimens examined: 13.iv.1967, S. L. Kapoor 70035 (LWG); 23.viii.2002, Nishangara range, coll. S.D. Maliya \& K.K. Singh 223611 (LWG) (Image 37).

Clerodendrum indicum (L.) Kuntze, Revis. Gen. PI. 2: 506. 1891; Panigrahi et al., Bull. Bot. Surv. India 11 (1 \& 2): 102. 1969; Maliya \& Datt, J. Econ. Taxon. Bot. 34 (1): 60. 2010

Siphonanthus indica L., Sp. PI. 109. 1753. Clerodendrum siphonanthus R. Br. in Ait., Hort. Kew 4: 65. 1812; Clarke in Hook. f., Fl. Brit. India 4: 595. 1885; Duthie, Fl. Upp. Gang. Plain 2: 93. repr. ed. 1960.

Habit: Shrub.

Specimens examined: 25.viii.2002, Murtiha range, coll. S.D. Maliya 223729 (LWG); 09.xi.2002, Near Bicchiya town, Katerniaghat range, coll. S.D. Maliya 224504 (LWG).

Clerodendrum inerme (L.) Gaertn., Fruct. Sem. PI. 1: 271. t. 75. 1788; Raizada, Suppl. Fl. Upp. Gang. Plain 212. 1976; Saini, J. Econ. Taxon. Bot. 29 (4): 853. 2005.

Volkameria inerme L., Sp. PI.637. 1753.
Habit: Shrub.

Specimens examined: Not observed. Record based on Saini (2005).

Clerodendrum viscosum Vent., Jard. Malm. 1: ad t. 25. 1803; Maliya \& Datt, J. Econ. Taxon. Bot. 34 (1): 60. 2010.

Habit: Shrub.

Specimens examined: 17.iv.2003, Katerniaghat range, Katerniaghat Wildlife Sanctuary, Bahraich coll. S.D. Maliya 224910 (LWG); 20.iv.2003, Murtiha range, Katerniaghat Wildlife Sanctuary, Bahraich coll. S.D. Maliya 224954 (LWG); 21.iv.2003, Katerniaghat range, Katerniaghat Wildlife Sanctuary, Bahraich coll. S.D. Maliya 224966 (LWG); 23.xii.2003, Girijapuri forest, coll. S.D. Maliya 225240 (LWG); 16.iii.2008, Murtiha range, coll. S.D. Maliya 218511 (LWG) (Image 38).

Colebrookea oppositifolia Sm., Exot. Bot. 2: 111, t. 115. 1850; Hook. f. in Hook. f., Fl. Brit. India 4: 642. 1885; Duthie, Fl. Upp. Gang. Plain 2: 107. repr. ed. 1960; Panigrahi et al., Bull. Bot. Surv. India 11 (1 \& 2): 108. 1969; Maliya \& Datt, J. Econ. Taxon. Bot. 34 (1): 60. 2010.

Habit: Shrub.

Specimens examined: 13.iv.1967, Katerniaghat range, S. L. Kapoor \& party 70048 (LWG); 09.xi.2002, Katerniaghat range, coll. S.D. Maliya 224548 (LWG); 17.iv.2003, Katerniaghat range, coll. S.D. Maliya 224924 (LWG); 24.xii.2003, Kutiaghat forest, coll. S.D. Maliya 225261 (LWG); 16.iii.2004, Karmohini forest, coll. S.D. Maliya 252283 (LWG); 16.iii.2008, Mutiha range, Katerniaghat Wildlife Sanctuary, Bahraich coll. S.D. Maliya 218510 (LWG) (Image 39).

Isodon coetsa (Buch.-Ham. ex D. Don) Kudo, Mem. Fac. Sci. Taihoku Imp. Univ. 2: 131. 1929.

Plectranthus coetsa Buch.-Ham. ex D. Don., Prodr. Fl.

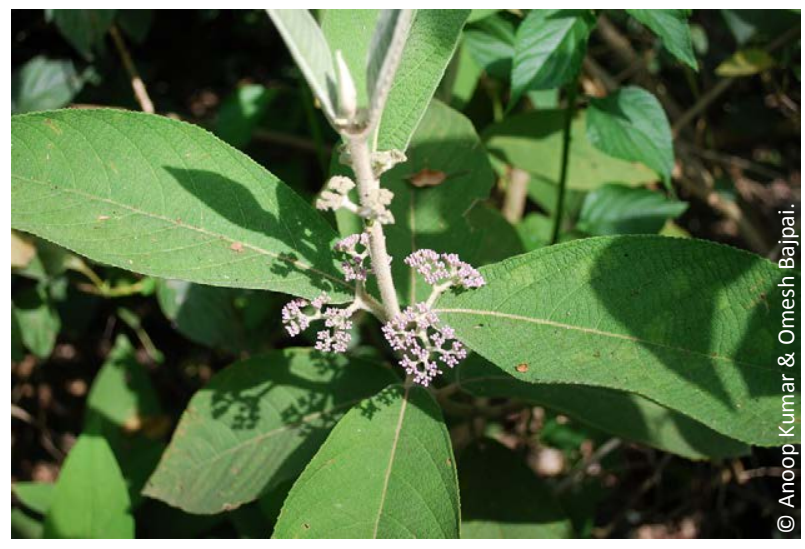

Image 37. Callicarpa macrophylla Vahl. 
Nepal. 117. 1825; Duthie, Fl. Upp. Gang. Plain 2: 103. repr. ed. 1960. Rabdosia coetsa (Buch. Ham. ex D. Don) Hara, J. Jap. Bot. 47: 194. 1972; Maliya \& Datt, J. Econ. Taxon. Bot. 34 (1): 61.2010.

Habit: Perennial herb.

Specimens examined: Not observed. Record based on Maliya \& Datt (2010).

**Isodon japonicus (Burm. f.) Hara, Enum. Spermatoph. Jap. 1: 206. 1948.

Scutellaria japonica Burm. f., Fl. Ind. 130. 1768. Plectranthus japonicus (Burm. f.) Koidz., Bot. Mag. (Tokyo) 43: 386. 1929.

Habit: Herb.

Specimens examined: 09.xi.2002, road side, Katerniaghat range, B. Datt \& coll. S.D. Maliya 224554 (LWG).

**Lantana camara L., Sp. PI. 627. 1753, nom. cons.; Maliya \& Datt, J. Econ. Taxon. Bot. 34 (1): 60. 2010.

L. aculeata L., Sp. PI. 627. 1753.

Habit: Shrub.

Specimens examined: 10.ii.2010, Amba village, L.B. Chaudhary, A. Kumar \& O. Bajpai 252195 (LWG).

Leonotis nepetifolia (L.) R. Br. in W. T. Aiton, Hort. Kew. 3: 409. 1811; Hook. f. in Hook. f., Fl. Brit. India 4: 691. 1885; Duthie, Fl. Upp. Gang. Plain 2: 116. repr. ed. 1960; Maliya \& Datt, J. Econ. Taxon. Bot. 34 (1): 60. 2010.

Phlomis nepetifolia L., Sp. PI. 586. 1753.

Habit: Annual herb.

Specimens examined: 22.xii.2005, Saryu link canal, Mihinpurva road, Katerniaghat Wildlife Sanctuary,

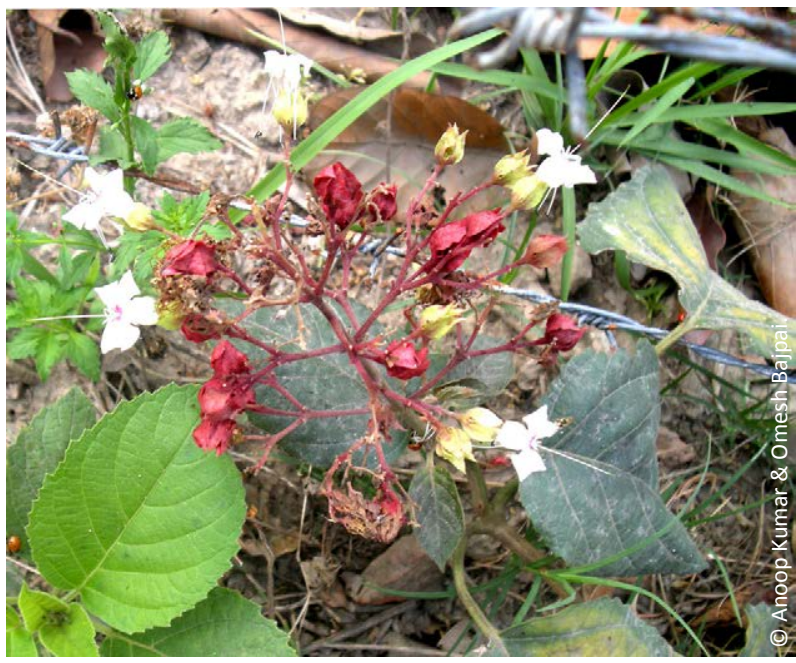

Image 38. Clerodendrum viscosum Vent.
Bahraich coll. S.D. Maliya 227173 (LWG).

Leucas aspera (Willd.) Link, Enum. Hort. Berol. Alt. 2: 113. 1822; Duthie, Fl. Upp. Gang. Plain 2: 113. repr. ed. 1960; Panigrahi et al., Bull. Bot. Surv. India 11 (1 \& 2): 108. 1969; Singh, Monogr. Indian Leucas R. Br. (Dronapushpi) Lamiaceae 43. 2001; Saini, J. Econ. Taxon. Bot. 29 (4): 857. 2005; Maliya \& Datt, J. Econ. Taxon. Bot. 34 (1): 60. 2010.

Phlomis aspera Willd., Enum. PI. 621. 1809.

Habit: Annual herb.

Specimens examined: Motipur range, O.P. Mishra 7725 (BSA); 09.ix.2005, Murtiha range, coll. S.D. Maliya 227169 (LWG).

Leucas cephalotes (Roth.) Spreng., Syst. Veg. 2: 743. 1825; Hook. f. in Hook. f., Fl. Brit. India 4: 689. 1885; Duthie, Fl. Upp. Gang. Plain 2: 114. repr. ed. 1960; Panigrahi et al., Bull. Bot. Surv. India 11 (1 \& 2): 108. 1969; Singh, Monogr. Indian Leucas R. Br. (Dronapushpi) Lamiaceae 53. 2001; Maliya \& Datt, J. Econ. Taxon. Bot. 34 (1): 60. 2010.

Phlomis cephalotes Roth., Nov. PI. Sp. 262. 1821.

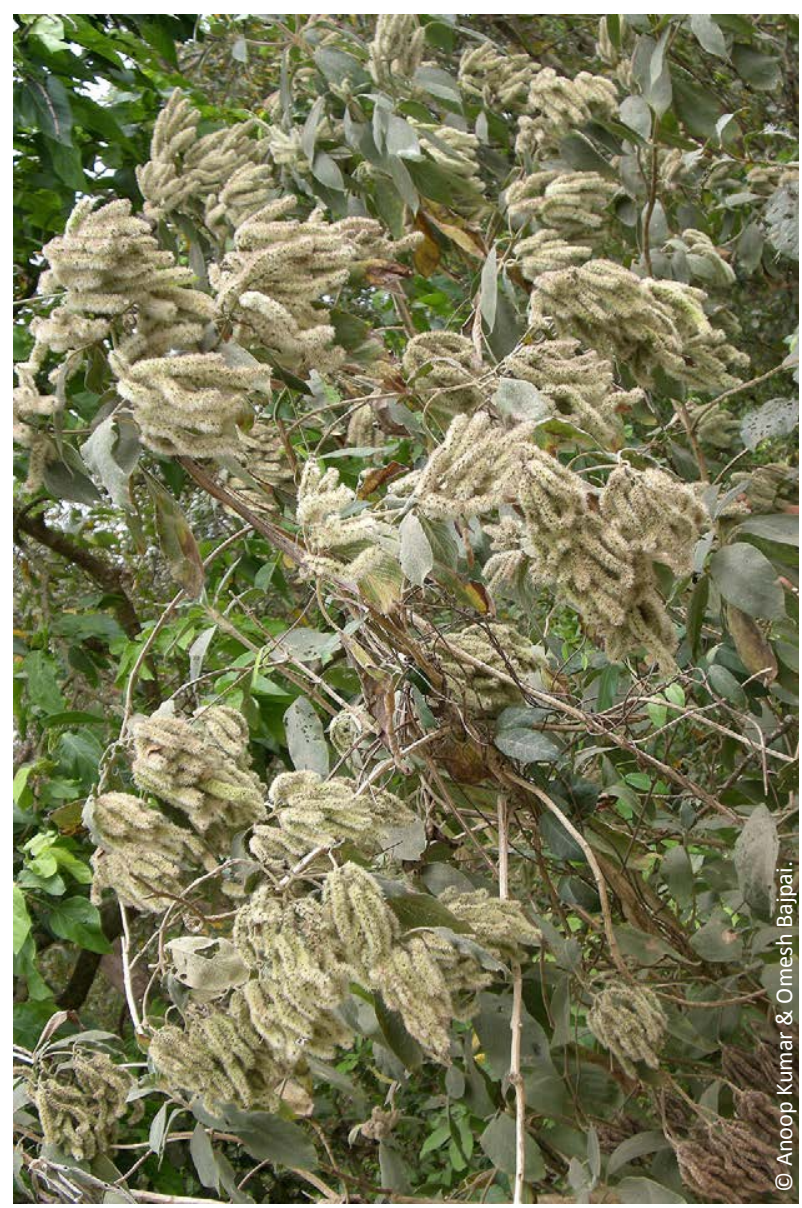

Image 39. Colebrookea oppositifolia Sm. 
Habit: Annual herb.

Specimens examined: 09.xi.2002, rest house, Katerniaghat range, coll. S.D. Maliya 242524 (LWG); 21.xii.2003, Katerniaghat range, coll. S.D. Maliya 225232 (LWG).

Leucas decemdentata (Willd.) Sm., Cycl. 20: 6. 1810; Singh, Monogr. Indian Leucas R. Br. (Dronapushpi) Lamiaceae 67. f. 10. 2001.

Phlomis decemdentata Willd., Sp. PI. 3: 124. 1800. Leucas montana var. pilosa Haines Bot. Bihar \& Orissa 4: 749. 1922. L. mollissima Wall. var. pilosa (Haines) Mukerjee, Rec. Bot. Surv. India 14 (1-2): 184. 1940; Saini, J. Econ. Taxon. Bot. 29 (4): 857. 2005.

Habit: Herb.

Specimens examined: Not observed. Record based on Saini (2005)

Nepeta graciliflora Benth., Pl. Asiat. Rar. 1: 65. 1830; Duthie, Fl. Upp. Gang. Plain 2: 118. repr. ed. 1960; Maliya \& Datt, J. Econ. Taxon. Bot. 34 (1): 60. 2010.

Habit: Annual herb.

Specimens examined: 24.xii.2003, Kuthiyaghat forest, coll. S.D. Maliya 225248 (LWG).

Nepeta hindostana (B. Heyne ex Roth) Haines, Bot. Bihar \& Orissa, 4: 744. 1922; Panigrahi et al., Bull. Bot. Surv. India 11 (1 \& 2): 108. 1969.

Glechoma hindostana B. Heyne ex Roth, Nov. PI. Sp. 258. 1821. Nepeta ruderalis Buch.-Ham. ex Benth. in Wall., PI. Asiat. Rar. 1: 64. 1830; Hook. f. in Hook. f., FI. Brit. India 4: 661. 1885; Duthie, Fl. Upp. Gang. Plain 2: 117. repr. ed. 1960.

Habit: Annual herb.

Specimens examined: 12.iii.1964, Murtiha gate, G. Panigrahi 2867 (BSA); 15.ii.1965, Girwa bridge, G. Panigrahi \& O.P. Mishra 8021 (BSA).

Ocimum americanum L., Cent. PI. 1: 15. 1755; Maliya \& Datt, J. Econ. Taxon. Bot. 34 (1): 60. 2010.

O. canum Sims. in Bot. Mag. 51: t. 2452. 1824; Hook. f. in Hook. f., FI. Brit. India 4: 607. 1885; Duthie, Fl. Upp. Gang. Plain 2: 99. repr. ed. 1960.

Habit: Herb.

Specimens examined: 23.viii.2002, Nishangara range, coll. S.D. Maliya 223661 (LWG).

** Ocimum basilicum L., Sp. PI. 597. 1753; Hook. f. in Hook. f., Fl. Brit. India 4: 608. 1885; Duthie, Fl. Upp. Gang. Plain 2: 99. repr. ed. 1960; Maliya \& Datt, J. Econ. Taxon. Bot. 34 (1): 60. 2010.
Habit: Herb, cultivation.

Specimens examined: 29.x.2004, Kutiyaghat forest, coll. S.D. Maliya 227135 (LWG).

Ocimum tenuiflorum L., Sp. PI. 597. 1753; Maliya \& Datt, J. Econ. Taxon. Bot. 34 (1): 60. 2010.

O. sanctum L., Mant. PI. 1: 85. 1767; Duthie, Fl. Upp. Gang. Plain 2: 98. repr. ed. 1960.

Habit: Herb, cultivation.

Specimens examined: 22.x.2004, Kuthiyaghat forest, coll. S.D. Maliya 225992 (LWG); 29.x.2004, Kuthiyaghat forest, coll. S.D. Maliya 227136 (LWG).

Orthosiphon pallidus Royle ex Benth., Hook. Bot. Misc. 3: 370. 1833; Hook. f. in Hook. f., Fl. Brit. India 613. 1885; Duthie, Fl. Upp. Gang. Plain 2: 100. repr. ed. 1960; Saini, J. Econ. Taxon. Bot. 29 (4): 859. 2005

Habit: Undershrub.

Specimens examined: Not observed. Record based on Saini (2005).

Perilla frutescens (L.) Britt., Mem. Torrey Bot. Club 5(18): 277. 1894; Maliya \& Datt, J. Econ. Taxon. Bot. 34 (1): 60. 2010.

P. ocimoides L., Gen. PI. ed. 6. 578. 1764. nom. illg. Habit: Herb.

Specimens examined: 09.xi.2002, road side, Katerniaghat range, B. Datt \& coll. S.D. Maliya 224557 (LWG).

**Phyla nodiflora (L.) Greene, Pittonia 4: 46. 1899; Meeuse, Blumea 5 (1): 69. 1942; Maliya \& Datt, J. Econ. Taxon. Bot. 34 (1): 60. 2010.

Verbena nodiflora L., Sp. PI. 20. 1753.

Habit: Creeping perennial herb.

Specimens examined: 22.iv.2003, Nishangara range, Katerniaghat Wildlife Sanctuary, Bahraich coll. S.D. Maliya 224993 (LWG).

*Plantago ovata Forssk., Fl. Aegypt.-Arab. 31. 1775; Hook. f. in Hook. f., Fl. Brit. India 4: 707. 1885; Duthie, Fl. Upp. Gang. Plain 2: 124. repr. ed. 1960; Saini, J. Econ. Taxon. Bot. 29(5): 860. 2005.

P. ispaghul Roxb. in J. Fleming, Asiat. Res. 11: 174. 1810.

Habit: Annual herb, cultivated.

Specimens examined: Not observed. Record based on Saini (2005).

Platostoma hispidum (L.) Paton, Kew Bull. 52: 273. 1997. 
Gomphrena hispida L., Sp. PI. 326. 1762. Acrocephalus capitatus Benth. in Wall., PI. As. Rar. 2: 28. 1831; Hook. f. in Hook. f., Fl. Brit. India 4: 611. 1885; Duthie, Fl. Upp. Gang. Plain 2: 102. repr. ed. 1960. Acrocephalus hispidus (L.) Nicolson \& Sivadasan, Taxon 29: 324. 1980; Maliya \& Datt, J. Econ. Taxon. Bot. 34 (1): 60. 2010.

Habit: Annual herb.

Specimens examined: Not observed. Record based on Maliya \& Datt (2010).

Pogostemon benghalensis (Burm. f.) Kuntze., Rev. Gen. PI. 2: 529. 1891; Raizada, Ind. For. 92. (5): 321. 1996; Panigrahi et al., Bull. Bot. Surv. India 11 (1 \& 2): 108. 1969; Saini, J. Econ. Taxon. Bot. 29 (4): 859. 2005; Maliya \& Datt, J. Econ. Taxon. Bot. 34 (1): 60. 2010.

Origanum benghalense Burm. f., Fl. Ind. 128, t. 38, f. 3. 1768. Pogostemon plectranthoides Desf., Ann. Mus. Natl. Hist. Nat. 2: 155. 1815; Hook. f. in Hook. f., Fl. Brit. India 4: 632. 1885; Duthie, Fl. Upp. Gang. Plain 2: 105. repr. ed. 1960.

Habit: Aromatic shrub.

Specimens examined: 08.ii.1965, Kharia nala, Motipur range, O.P. Mishra 7744 (BSA); 14.ii.1965, Urai tal, Bichhiya, Nishangara range, O.P. Mishra 7996 (BSA); 20.iv.2003, rest house, Murtiha range, coll. S.D. Maliya 224956 (LWG); 21.iv.2003, Nishangara range, coll. S.D. Maliya 224963 (LWG); 23.xii.2003, Girijapuri forest, coll. S.D. Maliya 225242 (LWG); 16.iii.2004, Karmohini forest, coll. S.D. Maliya 225296 (LWG); 17.iii.2004, Kuthiyaghat range, coll. S.D. Maliya 225902 (LWG); 29.xii.2005, Kuthiyaghat forest, coll. S.D. Maliya 226605 (LWG).

**Pogostemon pumilus (Graham) Press, Bull. Brit. Mus. (Nat. Hist.) Bot. 10: 74. 1982.

Mentha pumila Graham, Edinburgh New Philos. J. 4: 393. 1828. Dysophylla crassicaulis Benth., PI. Asiat. Rar. 1: 30. 1830; Duthie, Fl. Upp. Gang. Plain 2: 106. repr. ed. 1960; Panigrahi et al., Bull. Bot. Surv. India 11 (1 \& 2): 108. 1969.

Habit: Annual herb.

Specimens examined: 22.xi.1964, Motipur range, G. Panigrahi \& O.P. Mishra 6588 (BSA).

Pogostemon stellatus (Lour.) Kuntze, Revis. Gen. PI. 2: 429.1891.

Mentha stellata Lour., Fl. Cochinch. 2: 361-362. 1790. Dysophylla verticillata Benth., PI. Asiat. Rar. 1: 30 1830; Panigrahi et al., Bull. Bot. Surv. India 11 (1 \& 2): 108. 1969.

Habit: Herb.

Specimens examined: 21.xi.1964, Nishangara range, G. Panigrahi \& O.P. Mishra 6529 (BSA).
Salvia plebia R. Br., Prodr. 501. 1810; Hook. f. in Hook. f., Fl. Brit. India 4: 655. 1885; Duthie, FI. Upp. Gang. Plain 2: 118. repr. ed. 1960; Panigrahi et al., Bull. Bot. Surv. India 11 (1 \& 2): 108. 1969; Maliya \& Datt, J. Econ. Taxon. Bot. 34 (1): 61. 2010.

Habit: Annual herb.

Specimens examined: 26.v.1954, Motipur forest, Hira Lal 13424 (LWG); 21.iv.2003, Nishangara range, coll. S.D. Maliya 224961 (LWG); 18.iii.2004, Kuthiyaghat forest, coll. S.D. Maliya 225913 (LWG).

Tectona grandis L. f., Suppl. PI. 151. 1782; Clarke in Hook. f., Fl. Brit. India 4: 570. 1885; Duthie, Fl. Upp. Gang. Plain 2: 87. repr. ed. 1960; Panigrahi et al., Bull. Bot. Surv. India 11 (1 \& 2): 102. 1969; Maliya \& Datt, J. Econ. Taxon. Bot. 34 (1): 60. 2010.

Habit: Tree.

Specimens examined: 26.v.1954, Motipur range, Katerniaghat Wildlife Sanctuary, Bahraich Hira Lal 13451 (LWG); 23.viii.2002, Nishangara range, Katerniaghat Wildlife Sanctuary, Bahraich coll. S.D. Maliya 223673 (LWG); 26.viii.2002, Katerniaghat range, Katerniaghat Wildlife Sanctuary, Bahraich coll. S.D. Maliya 223755 (LWG).

**Verbena officinalis L., Sp. PI. 20. 1753; Clarke in Hook. f., Fl. Brit. India 4: 565. 1885; Duthie, Fl. Upp. Gang. Plain 2: 85. repr. ed. 1960; Saini, J. Econ.Taxon. Bot. 29 (4): 855. 2005.

Habit: Perennial herb.

Specimens examined: Not observed. Record based on Saini (2005).

\section{LAURACEAE}

Litsea glutinosa (Lour.) Rob., Philipp. J. Sci. 6(5): 321. 1911; Duthie, FI. Upp. Gang. Plain 3: 167. repr. ed. 1960. Sebifera glutinosa Lour., Fl. Cochinch. 2: 638. 1790. Habit: Tree.

Specimens examined: 13.xi.2011. Nishangara range, A. Kumar \& O. Bajpai 252787 (LWG).

Litsea monopetala (Roxb.) Pers., Syn. PI. 2(1): 4.1806. Tetranthera monopetala Roxb., PI. Coromandel 2: 26. 1798. Litsea polyantha Juss., Ann. Mus. Natl. Hist. Nat. 6: 211. 1805; Duthie, Fl. Upp. Gang. Plain 3: 168. repr. ed. 1960; Panigrahi et al., Bull. Bot. Surv. India 11 (1 \& 2): 93. 1969.

Habit: Tree.

Specimens examined: 14.iii.1964, Rampurwa, Katerniaghat Wildlife Sanctuary, Bahraich G. Panigrahi 2897 (BSA). 


\section{LECYTHIDACEAE}

Barringtonia acutangula (L.) Gaertn., Fruct. Sem. PI. 2: 97. t. 101. 1791; Clarke in Hook. f., Fl. Brit. India 2: 508. 1879; Duthie, Fl. Upp. Gang. Plain 1: 316. repr. ed. 1960; Saini, J. Econ. Taxon. Bot. 29 (3): 579. 2005; Maliya \& Datt, J. Econ. Taxon. Bot. 34 (1): 53. 2010.

Eugenia acutangula L., Sp. PI. 471. 1753.

Habit: Tree.

Specimens examined: 07.vi.2009, Near Lakkad Shah Baba Majar, Murtiha range, L.B. Chaudhary, A. Kumar \& O. Bajpai, 250605 (LWG); 31.x.2009, Beat no. 20, near Lakkad Shah Baba Majar, Murtiha range, L.B. Chaudhary, A. Kumar \& O. Bajpai, 252164 (LWG) (Image 40).

Careya arborea Roxb., Pl. Coromandel 3: 14, t. 218. 1819; Clarke in Hook. f., Fl. Brit. India 2: 511. 1879; Duthie, FI. Upp. Gang. Plain 1: 317. repr. ed. 1960; Saini, J. Econ. Taxon. Bot. 29 (3): 579. 2005.

Habit: Tree.

Specimens examined: 27.v.1954, Motipur range, Hira Lal 13470 (LWG).

\section{LENTIBULARIACEAE}

**Utricularia gibba L., Sp. PI.18. 1753.

U. exoleta R. Br., Prodr. 430. 1810; Clarke in Hook. f., FI. Brit. India 4: 329. 1884; Duthie, FI. Upp. Gang. Plain 2: 39. repr. ed. 1960; Panigrahi et al., Bull. Bot. Surv. India 11 (1 \& 2): 107. 1969.

Habit: Aquatic herb.

Specimens examined: 11.iii.1964, Motipur range, G. Panigrahi \& O.P. Mishra 2823 (BSA).

\section{LINACEAE}

*Linum usitatissimum L., Sp. PI. 277. 1753; Hook. f. in Hook. f., FI. Brit. India 1: 410. 1874; Duthie, Fl. Upp. Gang. Plain 1: 115. repr. ed. 1960; Hajra in Sharma et al., FI. India 3: 580. 1993; Saini, J. Econ. Taxon. Bot. 29 (3): 549. 2005; Maliya \& Datt, J. Econ. Taxon. Bot. 34 (1): 48. 2010.

Habit: Annual herb, cultivated.

Specimens examined: 21.xii.2003, Katerniaghat range, coll. S.D. Maliya 225235 (LWG).

\section{LINDERNIACEAE}

Lindernia anagallis (Burm. f.) Pennell, J. Arnold Arbor. 24 (3): 252. 1943; Philcox, Kew Bull. 17: 484. 1964; Saini, J. Econ. Taxon. Bot. 29 (3): 631. 2005; Maliya \& Datt, J. Econ. Taxon. Bot. 34 (1): 58. 2010.

Ruellia anagallis Burm. f., FI. Ind. 135. 1768. Vandellia pedunculata Benth., Scroph. Ind. 37. 1835; Duthie, FI. Upp. Gang. Plain 2: 24. repr. ed. 1960.

Habit: Decumbent or creeping herb.
Specimens examined: 10.xi.2002, Murtiha range, coll. S.D. Maliya 224576 (LWG); 30.viii.2003, rest house, Murtiha range, coll. S.D. Maliya 214867 (LWG).

Lindernia antipoda (L.) Alston in Trimen, Handb. Fl. Ceylon 6: 214. 1931; Philcox, Kew Bull. 17 (3): 484. 1964; Saini, J. Econ. Taxon. Bot. 29 (3): 632. 2005.

Ruellia antipoda L., Sp. PI. 635. 1753. Bonnaya veronicifolia (Retz.) Spreng, Syst. Veg. 1: 41. 1825; Hook. f. in Hook. f., Fl. Brit. India 4: 285. 1884; Duthie, Fl. Upp. Gang. Plain 2: 26. repr. ed. 1960.

Habit: Decumbent or creeping herb.

Specimens examined: Not observed. Record based on Saini (2005).

Lindernia ciliata (Colsm.) Pennell, J. Arnold Arbor. 24(3): 253. 1943 \& Scroph. West Himal., 32. 1943; Saini, J. Econ. Taxon. Bot. 29 (3): 632. 2005; Maliya \& Datt, J. Econ. Taxon. Bot. 34 (1): 58. 2010.

Gratiola ciliata Colsm., Prodr. Descr. Grat. 14. 1793. Bonnaya brachiata Link \& Otto, Icon. PI. select, 25, t. 11. 1821; Hook. f. in Hook. f., Fl. Brit. India 4: 284. 1884; Duthie, Fl. Upp. Gang. Plain 2: 26. repr. ed. 1960.

Habit: Annual herb.

Specimens examined: 23.viii.2002, Nishangara range, coll. S.D. Maliya 223646 (LWG).

**Lindernia crustacea (L.) F. Muell., Syst. Census Austral. PI. 97. 1882; Maliya \& Datt, J. Econ. Taxon. Bot. 34 (1): 59. 2010.

Capraria crustacea L., Mant. PI. 1: 87. 1767. Vandellia crustacea (L.) Benth., Scroph. Ind. 35. 1835; Hook. f. in

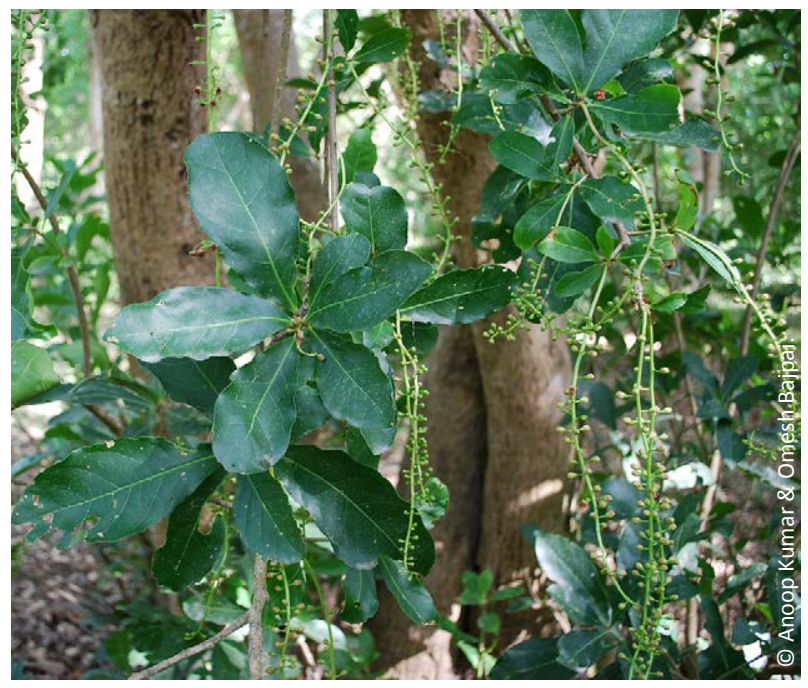

Image 40. Barringtonia acutangula (L.) Gaertn. 
Hook. f., Fl. Brit. India 4: 279. 1884; Duthie, FI. Upp. Gang. Plain 2: 23. repr. ed. 1960.

Habit: Annual herb.

Specimens examined: 09.xi.2002, road side, Katerniaghat range, coll. S.D. Maliya 224567 (LWG); 22.ii.2005, Kuthiyaghat forest, coll. S.D. Maliya 224681 (LWG).

** Lindernia nummulariifolia (D. Don) Wettst. in Engler \& Prantl, Nat. Pflanzenfam. 4(3b): 79. 1891; Maliya \& Datt, J. Econ. Taxon. Bot. 34 (1): 59. 2010.

Vandellia nummulariifolia D. Don., Prodr. Fl. Nepal. 86. 1825

Habit: Herb.

Specimens examined: 23.viii.2002, Nishangara range, coll. S.D. Maliya 223637 (LWG); 25.viii.2002, rest house, Murtiha range, coll. S.D. Maliya 223743 (LWG); 09.xi.2002, rest house, Katerniaghat range, coll. S.D. Maliya 224531 (LWG); 07.x.2009, rest house, Kakraha range, coll. S.D. Maliya 226656 (LWG).

Lindernia procumbens (Krock.) Philcox, Taxon 14: 30. 1965.

Anagalloides procumbens Krock., Fl. Siles. 2: 398. 1790. Lindernia pyxidaria L., Melanges Philos. Math. Soc. Roy. Turin 3: 181. 1766; Pennell in Acad. Nat. Sci. Philad. Monog. 5: 30. 1943; Raizada, Suppl. Fl. Upp. Gang. Plain 185. 1976; Saini, J. Econ. Taxon. Bot. 29 (3): 633. 2005. Vandellia erecta Benth., Scroph. Ind. 36. 1835; Hook. f. in Hook. f.,Fl. Brit. India 4: 281. 1884.

Habit: Annual herb.

Specimens examined: Not observed. Record based on Saini (2005).

Lindernia viscosa (Hornem.) Merr., Sp. Blancoan. 14. 1918.

Gratiola viscosa Hornem., Enum. PI. Hort. Hafn. (Rev. ed.) 19. 1807. Lindernia viscosa Bold., Zakfl. Java 165. 1916. (Invalid name).

Habit: Annual herb.

Specimens examined: 23.viii.2002, Nishangara range, coll. S.D. Maliya 223636 (LWG); 24.viii.2002, Nishangara range, coll. S.D. Maliya 223677 (LWG).

\section{LORANTHACEAE}

Dendrophthoe falcata (L. f.) Ettingsh., Denkschr. Kaiserl. Akad. Wiss, Wien. Math.- Naturwiss. KI. 32: 52. 1872; Panigrahi et al., Bull. Bot. Surv. India 11 (1 \& 2): 100. 1969; Saini, J. Econ. Taxon. Bot. 29 (4): 868. 2005; Maliya \& Datt, J. Econ. Taxon. Bot. 34 (1): 62. 2010.

Loranthus falcatus L. f., Suppl. PI. 211. 1781. L. longiflorus Desr. in Lam., Encycl. Meth. B. 3: 598. 1789; Hook. f. in Hook. f., Fl. Brit. India 5: 214. 1886; Duthie, Fl. Upp. Gang. Plain 3: 175. repr. ed. 1960.

Habit: Parasitic shrub.

Specimens examined: 20.xi.1964, Nishangara range, G. Panigrahi \& O.P. Mishra 6487 (BSA); 23.xii.2003, Nishangara range, coll. S.D. Maliya 225244 (LWG) (Image 41).

\section{LYTHRACEAE}

Ammannia auriculata Willd., Hort. Berol. 1: 7, t. 7. 1806; Saini, J. Econ. Taxon. Bot. 29 (3): 579. 2005.

A. senegalensis DC., Prodr. 3: 77. 1828; Clarke in Hook. f., Fl. Brit. India 2: 570. 1879.

Habit: Herb.

Specimens examined: Not observed. Record based on Saini (2005).

Ammannia baccifera L., Sp. Pl. 120. 1753; Clarke in Hook. f., Fl. Brit. India 2: 569. 1879; Duthie, Fl. Upp. Gang. Plain 1: 321. repr. ed. 1960; Maliya \& Datt, J. Econ. Taxon. Bot. 34 (1): 53. 2010.

Habit: Herb.

Specimens examined: 26.x.2004, Murtiha range, coll. S.D. Maliya 227117 (LWG); 29.x.2004, Kuthiyaghat forest, coll. S.D. Maliya 227140 (LWG).

Ammannia multiflora Roxb., Fl. Ind., 1: 447. 1820; Maliya, J. Econ. Taxon. Bot. 36 (1): 157. 2012 \& 36 (2): 421. 2012.

Habit: Herb.

Specimens examined: Not observed. Record based on Maliya \& Datt (2012).

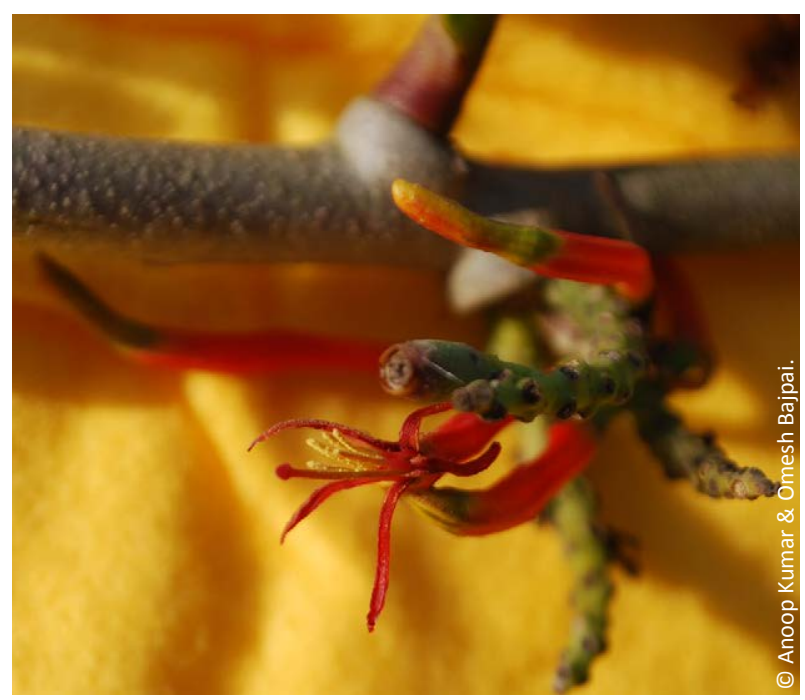

Image 41. Dendrophthoe falcata (L. f.) Ettingsh. 
Lagerstroemia parviflora Roxb., Pl. Coromandel 1: 47. t. 66. 1796; Clarke in Hook. f., FI. Brit. India 2: 575. 1879; Duthie, Fl. Upp. Gang. Plain 1: 324. repr. ed. 1960; Panigrahi et al., Bull. Bot. Surv. India 11 (1 \& 2): 104. 1969; Maliya \& Datt, J. Econ. Taxon. Bot. 34 (1): 53. 2010.

Habit: Tree.

Specimens examined: 09.xii.1986, Katerniaghat range, K.K. Khanna \& R. Saran 37687 (BSA); 20.v.1990, Murtiha range, K.K. Khanna 39896 (BSA); 12.xi.2002, Nishangara range, coll. S.D. Maliya 224625 (LWG); 23.viii.2002, Nishangara range, coll. S.D. Maliya 223655 (LWG); 24.xii.2003, Kuthiyaghat forest, coll. S.D. Maliya 225249 (LWG); 20.vii.2004, Girijapuri forest, coll. S.D. Maliya 225970 (LWG); 22.ii.2009, near Balcha barrier, Kakraha range, L.B. Chaudhary, A. Kumar \& O. Bajpai 250263 (LWG); 27.iv.2010, Kakraha range, L.B. Chaudhary, A. Kumar \& O. Bajpai 252806 (LWG) (Image 42).

Lagerstroemia speciosa (L. ex Murray) Pers., Syn. 2: 72. 1806; Merr., J. Arn. Arb. 25: 146. 1954; Maliya \& Datt, J. Econ. Taxon. Bot. 34 (1): 53. 2010.

Munchhausai speciosa L., Muench. Hausb. 5(1): 357, t. 2: 1770 \& Mant. PI. 2: 243. 1771. Lagerstroemia flosreginae Retz., Observ. Bot. 5: 25. 1789; Clarke in Hook. f., FI. Brit. India 2: 577. 1879. pp.; Duthie, Fl. Upp. Gang. Plain 1: 325. repr. ed. 1960.

Habit: Tree.

Specimens examined: 20.iv.2005, Near Bichhiya on Bisunpura road, coll. S.D. Maliya 224687 (LWG); 26.iv.2010, rest house, Nishangara range, L.B. Chaudhary, A. Kumar \& O. Bajpai 252295 (LWG).

*Punica granatum L., Sp. Pl. 1: 472. 1753.

Habit: Shrub or small tree, cultivated.

Specimens examined: 21.v.1987, K.K. Singh \& party 5976 (LWG).

Rotala indica (Willd.) Koehne, Bot. Jahrb. 1: 172. 1880; Saini, J. Econ. Taxon. Bot. 29 (3): 580. 2005.

Peplis indica Willd., Sp. PI. 2: 244. 1799. Ammannia peploides Spreng., Syst. Veg. 1: 444. 1825; Clarke in Hook. f., Fl. Brit. India 2: 566. 1879; Duthie, Fl. Upp. Gang. Plain 1: 320. repr. ed. 1960

Habit: Herb.

Specimens examined: Not observed. Record based on Saini (2005).

Rotala mexicana Schltdl. \& Cham., Linnaea 5: 567. 1830.

Habit: Herb.

Specimens examined: 08.xii.1986, Bichhiya, K.K.
Khanna \& R. Saran 37631 (BSA).

Rotala occultiflora Koehne, Bot. Jahrb. Syst. 1(2): 152. 1880; Panigrahi et al., Bull. Bot. Surv. India 11 (1 \& 2): 104. 1969.

Habit: Herb.

Specimens examined: 20.xi.1964, Nishangara range, G. Panigrahi \& O.P. Mishra 6452 (BSA).

** Rotala rotundifolia (Buch.-Ham. ex Roxb.) Koehne, Bot. Jahrb. 1: 175. 1881; Panigrahi et al., Bull. Bot. Surv. India 11 (1 \& 2): 104. 1969.

Ammannia rotundifolia Buch.-Ham. ex Roxb., Fl. Ind. 1: 446. 1820; D. Don, Fl. Nepal. 220. 1825; Clarke in Hook.

f., Fl. Brit. India 2: 266. 1879.

Habit: Herb.

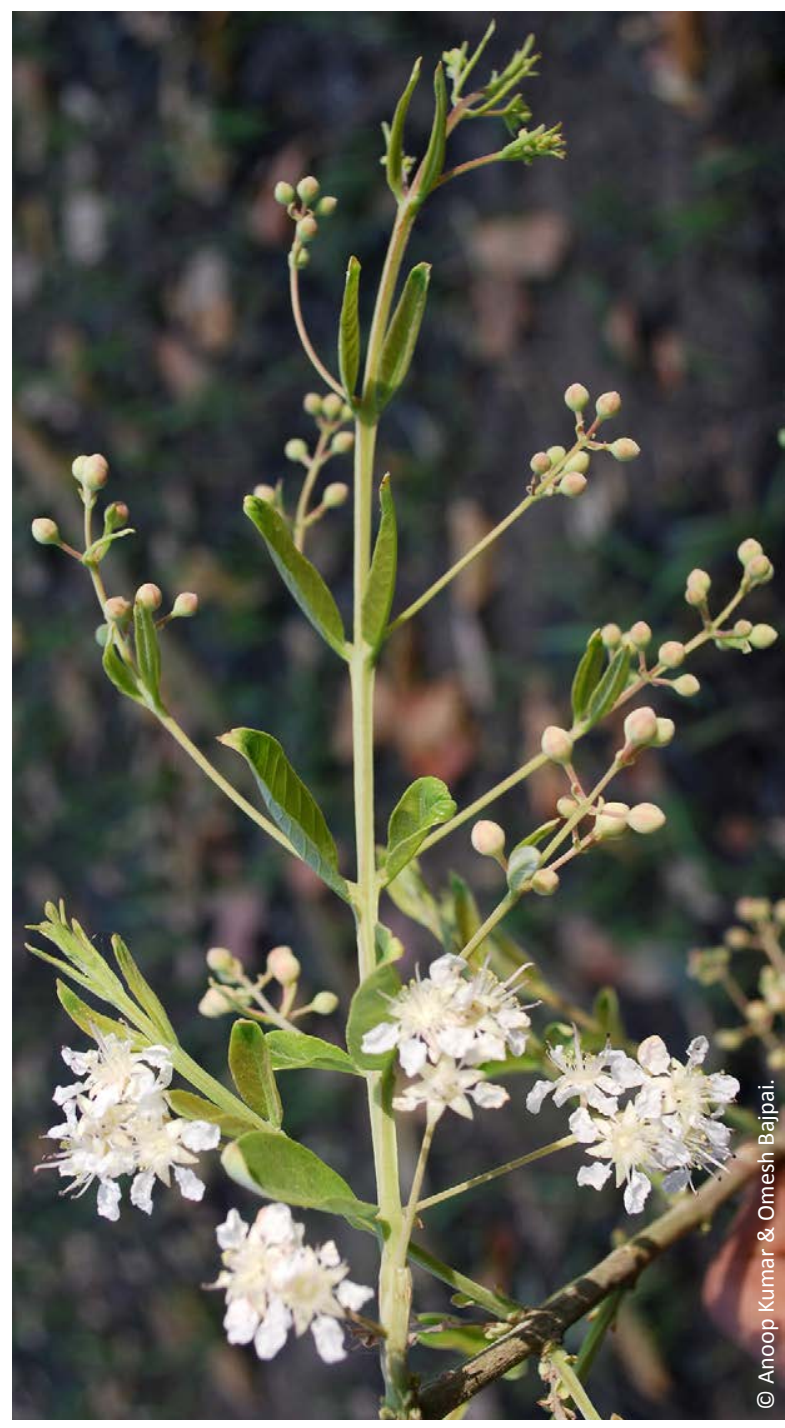

Image 42. Lagerstroemia parviflora Roxb. 
Specimens examined: Not observed. Record based on Panigrahi et al. (1969).

Trapa natans Linn. var. bispinosa (Roxb.) Makino in Bot. Mag. (Tokyo) 11: 283. 1897; Santapau \& Wagh, Bull. Bot. Surv. India 5 (2): 109. 1963; Panigrahi et al., Bull. Bot. Surv. India 11 (1 \& 2): 104. 1969.

T. bispinosa Roxb., Pl. Coromandel 3: t. 234. 1819; Clarke in Hook. f., Fl. Brit. India 2: 590. 1879; Duthie, FI. Upp. Gang. Plain 1: 329. repr. ed. 1960.

Habit: Aquatic herb.

Specimens examined: 22.xi.1964, Motipur on the way to Orla, G. Panigrahi \& O.P. Mishra 6553 (BSA).

Woodfordia fruticosa (L.) Kurz, J. Asiat. Soc. Bengal Pt. 2, Nat. Hist. 40(2): 56. 1871; Maliya \& Datt, J. Econ. Taxon. Bot. 34 (1): 53. 2010.

Lythrum fruticosum L., Syst. Nat. (ed. 10) 2: 1045. 1759 \& Sp. PI. 641. 1762. Woodfordia floribunda Salisb., Parad. London 1: 2, t. 42. 1806; Clarke in Hook., f., Fl. Brit. India 2: 572. 1879; Duthie, Fl. Upp. Gang. Plain 1: 323. repr. ed. 1960.

Habit: Shrub or small tree.

Specimens examined: 18.ii.1965, Kauriala nala, O.P. Mishra 8032 (BSA); 21.iv.2003, Nishangara range, coll. S.D. Maliya 224965 (LWG); 27.xii.2003, Nishangara range, coll. S.D. Maliya 225275 (LWG); 17.iii.2004, Kuthiyaghat forest, coll. S.D. Maliya 225300 (LWG); 04.iv.2011, near Gerwa river, A. Kumar, O. Bajpai \& K. Kishor 263633 (LWG).

\section{MALPIGHIACEAE}

Aspidopterys wallichii Hook. f. in Hook. f., Fl. Brit. India1: 421. 1874; Srivastava in Sharma et al., Fl. India 4: 12. 1997; Saini, J. Econ. Taxon. Bot. 29 (3): 549. 2005.

Habit: Climber.

Specimens examined: Not observed. Record based on Saini (2005).

Hiptage benghalensis (L.) Kurz, J. Asiat. Soc. Bengal. Pt. 2, Nat. Hist., 14: 136. 1874; Srivastava in Sharma et al., Fl. India 4: 14. 1997; Saini, J. Econ. Taxon. Bot. 29 (3): 549. 2005.

Benisteria benghalensis L., Sp. PI. 427. 1753. Hiptage madablota Gaertn., Fruct. 2: 169, t. 116. 1791; Hook. f. in Hook. f., Fl. Brit. India 1: 418. 1874.

Habit: Woody climber.

Specimens examined: Not observed. Record based on Saini (2005).

\section{MaLvaceae}

*Abelmoschus esculentus (L.) Moench, Methodus 2: 617. 1794; Saini, J. Econ. Taxon. Bot. 29 (3): 542. 2005; Maliya \& Datt, J. Econ. Taxon. Bot. 34 (1): 47. 2010.

Hibiscus esculantus L., Sp. PI. 696. 1753; Mast. in Hook. f., Fl. Brit. India 1: 343. 1874; Duthie, Fl. Upp. Gang. Plain 1: 87. repr. ed. 1960.

Habit: Herb, cultivated

Specimens examined: 19.vii.2004, Vardia village, coll. S.D. Maliya 225981 (LWG).

*Abelmoschus manihot (L.) Medik. subsp. tetraphyllus (Roxb. ex Hornem.) Borss. Waalk. var. pungens (Roxb.) Hochr., Candollea 2: 87. 1924; Paul in Sharma et al., FI. India 3: 307. 1993.

Hibiscus pungens Roxb. (Hort. Beng. 52. 1814, nom. nud.) Fl. Ind. 3: 213. 1832. H. manihot L. var. pungens (Roxb.) Hochr., Ann. Cons. Jard. Bot. Geneve 4: 155. 1900. Abelmoschus manihot (L.) Medik. var. pungens (Roxb.) Hochr., Ann. Cons. Jard. Bot. Geneve 4: 155. 1900; Panigrahi et al., Bull. Bot. Surv. India 11 (1 \& 2): 98.1969.

Habit: Herb, cultivated.

Specimens examined: 23.xi.1964, Motipur, G. Panigrahi \& O.P. Mishra 6590 (BSA).

Abutilon indicum (L.) Sweet, Hort. Brit. 54. 1826; Mast. in Hook. f., Fl. Brit. India 1: 326. 1874; Duthie, Fl. Upp. Gang. Plain 1: 78. repr. ed. 1960; Panigrahi et al., Bull. Bot. Surv. India 11 (1 \& 2): 98. 1969; Paul in Sharma et al., Fl. India 3: 266. 1993.

Sida indica L., Cent. PI. 2: 26. 1756.

Habit: Undershrub.

Specimens examined: Not observed. Record based on Panigrahi et al. (1969).

Abutilon ramosum (Cav.) Guill. \& Perr., Fl. Seneg. Tent. 1: 68. 1831; Duthie, Fl. Upp. Gang. Plain 1: 79. repr. ed. 1960; Paul in Sharma et al., FI. India 3: 271. 1993; Maliya \& Datt, J. Econ. Taxon. Bot. 34 (1): 47. 2010.

Sida ramosa Cav., Diss. 1: 28, t. 6. f. 1. 1785.

Habit: Shrub.

Specimens examined: 19.vii.2004, Kuthiyaghat, coll. S.D. Maliya 225977 (LWG)

Corchorus aestuans L., Syst. Nat. 2: 1079. 1759; Daniel \& Chandrabose in Sharma et al., Fl. India 3: 485. 1993; Maliya \& Datt, J. Econ. Taxon. Bot. 34 (1): 48. 2010.

C. acutangulus Lam., Encycl. 2: 104. 1786 (auct. non Forsk., 1775); Mast. in Hook. f., Fl. Brit. India 1: 398. 1874; Duthie, Fl. Upp. Gang. Plain 1: 114. repr. ed. 1960.

Habit: Herb. 
Specimens examined: 29.x.2004, Kuthiyaghat forest, coll. S.D. Maliya 227141 (LWG); 29.x.2004, Kuthiyaghat forest, coll. S.D. Maliya 227143 (LWG); 08.ix.2005, Kuthiyaghat forest, coll. S.D. Maliya 227165 (LWG); 07.x.2009, rest house, Kakraha range, coll. S.D. Maliya 226657 (LWG).

**Corchorus capsularis L., Sp. PI. 529. 1753; Mast. in Hook. f., Fl. Brit. India 1: 397. 1874; Duthie, Fl. Upp. Gang. Plain 1: 113. repr. ed. 1960; Daniel \& Chandrabose in Sharma et al., FI. India 3: 485. 1993; Saini, J. Econ. Taxon. Bot. 29 (3): 547. 2005.

Habit: Herb.

Specimens examined: Not observed. Record based on Saini (2005)

Corchorus fascicularis Lam., Encycl. 2: 104. 1786; Mast. in Hook. f., Fl. Brit. India 1: 398. 1874; Daniel \& Chandrabose in Sharma et al., Fl. India 3:486. 1993; Saini, J. Econ. Taxon. Bot. 29 (3): 547. 2005.

Habit: Herb.

Specimens examined: Not observed. Record based on Saini (2005).

Corchorus olitorius L., Sp. Pl. 529. 1753; Mast. in Hook. f., Fl. Brit. India 1: 397. 1874; Duthie, Fl. Upp. Gang. Plain 1: 113. repr. ed. 1960; Daniel \& Chandrabose in Sharma et al., Fl. India 3: 487. 1993; Saini, J. Econ. Taxon. Bota. 29 (3): 547. 2005; Maliya \& Datt, J. Econ. Taxon. Bot. 34 (1): 48. 2010.

Habit: Herb.

Specimens examined: 22.viii.2002, Murtiha range, coll. S.D. Maliya 223713 (LWG); 23.viii.2002, Nishangara range, coll. S.D. Maliya 223663 (LWG).

Corchorus tridens L., Mant PI. 2: 566. 1771; Mast. in Hook. f., Fl. Brit. India 1: 398. 1874; Duthie, Fl. Upp. Gang. Plain 1: 114. repr. ed. 1960; Daniel \& Chandrabose in Sharma et al., Fl. India 3: 488. 1993; Saini, J. Econ. Taxon. Bot. 29 (3): 547. 2005

Habit: Herb.

Specimens examined: Not observed. Record based on Saini (2005)

Grewia abutilifolia Vent. ex Juss., Ann. Mus. Natl. Hist. Nat. 4: 921804.

Habit: Tree.

Specimens examined: 20.v.1990, Murtiha range, K.K. Khanna 39907 (BSA).

Grewia asiatica L., Mant. PI. 1: 122. 1767 \&Syst. nat. ed. 12, 2:602. 1767; Mast. in Hook. f., Fl. Brit. India 1: 386. 1874; Duthie, Fl. Upp. Gang. Plain 1: 62. repr. ed. 1960; Panigrahi et al., Bull. Bot. Surv. India 11 (1 \& 2): 97.1969; Daniel \& Chandrabose in Sharma et al., FI. India 3: 494. 1993; Saini, J. Econ. Taxon. Bot. 29 (3): 548. 2005.

Habit: Tree.

Specimens examined: 16.ii.1965, Katerniaghat range, O.P. Mishra 8009 (BSA).

Grewia helicterifolia Wall. ex G. Don, Gen. Hist. 1: 548. 1831.

Habit: Shrub or small tree.

Specimens examined: 09.xii.1986, K.K. Khanna \& R. Saran 37659 (BSA); 12.xii.1986, Motipur range, K.K. Khanna \& R. Saran 38656 (BSA).

Grewia hirsuta Vahl, Symb. Bot. 1: 34. 1790; Mast. in Hook. f., Fl. Brit. India 1: 391. 1874; Panigrahi et al., Bull. Bot. Surv. India 11 (1 \& 2): 98. 1969; Daniel \& Chandrabose in Sharma et al., FI. India 3: 501. 1993; Maliya \& Datt, J. Econ. Taxon. Bot. 34 (1): 48. 2010.

Habit: Shrub.

Specimens examined: 21.xi.1964, Nishangara range, G. Panigrahi \& O.P. Mishra 6510 (BSA); 21.v.1990, Kakraha range, K.K. Khanna 66 (BSA); 23.viii.2002, Nishangara range, coll. S.D. Maliya 223643 (LWG); 24.viii.2002, Nishangara range, coll. S.D. Maliya 223685; 09.xii.2002, near Bichhiya town railway track, coll. S.D. Maliya 224514 (LWG); 25.xii.2003, Nishangara range, coll. S.D. Maliya 225266 (LWG).

Grewia multiflora Juss., Ann. Mus. Natl. Hist. Nat. 4: 89. 1804.

G. serrulata DC., Prodr. 1: 510. 1824; Daniel \& Chandrabose in Sharma et al., Fl. India 3: 509. 1993; Maliya \& Datt, J. Econ. Taxon. Bot. 34 (1): 48. 2010. G. disperma Rottler ex Spreng., Syst. Veg. 2: 579. 1825; Saini, J. Econ. Taxon. Bot. 29 (3): 548. 2005. G. barberi Drumm. ex Dunn in Gamble, Fl. Madras 115. 1915; Panigrahi et al., Bull. Bot. Surv. India 11 (1 \& 2): 98. 1969.

Habit: Shrub or small tree.

Specimens examined: 11.ii.1965, Bichhia, O.P. Mishra 7916 (BSA); 08.xii.1986, Bichhiya, K.K. Khanna \& R. Saran 37650 (BSA); 02.ix.2003, Kutiyaghat forest, coll. S.D. Maliya 225205 (LWG); 22.x.2004, Kuthiyaghat forest, coll. S.D. Maliya 225988 (LWG); 22.x.2004, Kuthiyaghat forest, coll. S.D. Maliya 225991 (LWG).

Grewia optiva Dumm. ex Burret., Notizbl. Bot. Gart. Berlin-Dahlem 9: 692. 1926; Daniel \& Chandrabose in Sharma et al., Fl. India 3: 504. 1993; Maliya \& Datt, J. 
Econ. Taxon. Bot. 35 (1): 36. 2011.

Habit: Small tree or shrub.

Specimens examined: Not observed. Record based on Maliya \& Datt (2011).

Grewia rothii DC., Prodr. 1: 509. 1824; Daniel \& Chandrabose in Sharma et al., Fl. India 3: 507. 1993; Saini, J. Econ. Taxon. Bota. 29 (3): 548. 2005.

Habit: Small shrub.

Specimens examined: Not observed. Record based on Saini (2005).

Grewia sapida Roxb. ex DC., Prodr. 1: 512. 1824; Roxb., Fl. Ind. 2: 590. 1832; Mast in Hook. f., Fl. Brit. India 1: 387. 1874; Daniel \& Chandrabose in Sharma et al., FI. India 3: 508. 1993; Saini, J. Econ. Taxon. Bot. 29 (3): 548. 2005; Maliya \& Datt, J. Econ. Taxon. Bot. 34 (1): 48. 2010.

Grewia pumila Buch.-Ham. ex D.Don, Prodr. Fl. Nepal. 227. 1825

Habit: Undershrub.

Specimens examined: 17.iv.2003, Katerniaghat range, coll. S.D. Maliya 224911 (LWG); 19.iv.2003, Murtiha range, coll. S.D. Maliya 224938 (LWG); 21.iv.2003, Nishangara range, coll. S.D. Maliya 224964 (LWG).

**Grewia sclerophylla Roxb. ex G. Don, Gen. Hist. 1: 550. 1831; Daniel \& Chandrabose in Sharma et al., Fl. India 3: 509. 1993; Maliya \& Datt, J. Econ. Taxon. Bot. 34 (1): 48. 2010.

G. scabrophylla Roxb., Fl. Ind. 2: 584. 1832.

Habit: Small shrub.

Specimens examined: 22.iv.2003, Nishangara range, coll. S.D. Maliya 224974 (LWG); 29.viii.2003, Nishangara range, coll. S.D. Maliya 214862 (LWG).

Grewia tillifolia Vahl, Symb. Bot. 1: 35. 1790; Daniel \& Chandrabose in Sharma et al., Fl. India 3: 511. 1993; Maliya \& Datt, J. Econ. Taxon. Bot. 34 (1): 48. 2010.

Habit: Tree.

Specimens examined: 19.vii.2004, Kuthiyaghat forest, coll. S.D. Maliya 225964 (LWG); 22.x.2004, Kuthiyaghat forest, coll. S.D. Maliya 225987 (LWG); 29.x.2004, Girijapuri forest, coll. S.D. Maliya 227145 (LWG).

*Hibiscus mutabilis L., Sp. PI. 694. 1753; Mast. in Hook. f., Fl. Brit. India 1: 344. 1874; Paul in Sharma et al., Fl. India 3: 390. 1993; Saini, J. Econ. Taxon. Bot. 29 (3): 544. 2005.

Habit: Shrub, cultivated.

Specimens examined: Not observed. Record based on Saini (2005).
*Hibiscus radiatus Cav., Diss. 3:150, t. 54, f. 2. 1787.

Habit: Herb, cultivated.

Specimens examined: 29.x.2004, Kuthiyaghat forest, coll. S.D. Maliya 227142 (LWG).

*Hibiscus rosa-sinensis L., Sp. PI. 694. 1753; Mast. in Hook. f., Fl. Brit. India 1: 344. 1874; Paul in Sharma et al., FI. India 3: 391. 1993; Maliya \& Datt, J. Econ. Taxon. Bot. 34 (1): 47. 2010.

Habit: Shrub or small tree, cultivated.

Specimens examined: 31.viii.2003, Bisunpura village, coll. S.D. Maliya 214887 (LWG).

*Hibiscus sabdariffa L., Sp. Pl. 695. 1753, nom. cons.; Mast. in Hook. f., Fl. Brit. India 1: 340. 1874; Paul in Sharma et al., Fl. India 3: 391. 1993; Maliya \& Datt, J. Econ. Taxon. Bot. 34 (1): 47. 2010.

Sabdariffa rubra Kostel., Allg. Med.- Pharm. Fl. 5: 1857. 1836

Habit: Herb, cultivated.

Specimens examined: 27.xii.2005, Lahura village, Nishangara range, coll. S.D. Maliya 227193 (LWG).

**Hibiscus vitifolius L., Sp. Pl. 696. 1753; Panigrahi et al., Bull. Bot. Surv. India 11 (1 \& 2): 98. 1969.

Habit: Herb.

Specimens examined: 21.xi.1964, Nishangara range, G. Panigrahi \& O.P. Mishra 6511 (BSA).

Kydia calycina Roxb., Pl. Coromandel 3: 12, t. 215. 1819; Mast. in Hook. f., Fl. Brit. India 1: 348; Panigrahi et al., Bull. Bot. Surv. India 11 (1 \& 2): 98. 1969; Paul in Sharma et al., Fl. India 3: 344. 1993; Maliya \& Datt, J. Econ. Taxon. Bot. 34 (1): 47. 2010.

K. fraterna Roxb., PI. Coromandel 3: 12, t. 216. 1819. Habit: Tree.

Specimens examined: 24.xi.1964, Nishangara range, G. Panigrahi \& O.P. Mishra 6602 (BSA); 12.ii.1965, Rampurwa, Nishangara range, G. Panigrahi \& O.P. Mishra 7961 (BSA); 11.xi.2002, Murtiha range, coll. S.D. Maliya 224603 (LWG); 24.xii.2003, Kuthiyaghat forest, coll. S.D. Maliya 225245 (LWG); 22.x.2004, Kuthiyaghat forest, coll. S.D. Maliya 225998 (LWG); 26.x.2009, Nishangara to Katerniaghat road, L.B. Chaudhary, A. Kumar \& O. Bajpai 252127 (LWG); 26.x.2009, Nishangara to Katerniaghat road, L.B. Chaudhary, A. Kumar \& O. Bajpai 252130 (LWG); 26.x.2009, Nishangara to Katerniaghat road, L.B. Chaudhary, A. Kumar \& O. Bajpai 252131 (LWG).

**Malva parviflora L., Demonstr. PI. 18. 1753; Mast. in Hook. f., Fl. Brit. India. 1: 321. 1874; Duthie, Fl. Upp. 
Gang. Plain 1: 74. repr. ed. 1960; Panigrahi et al., Bull. Bot. Surv. India 11 (1 \& 2): 98. 1969; Paul in Sharma et al., Fl. India 3: 361. 1993; Saini, J. Econ. Taxon. Bot. 29 (3): 544. 2005.

Habit: Herb.

Specimens examined: Not observed. Record based on Panigrahi et al. (1969) and Saini (2005).

Malvastrum coromandelianum (L.) Garcke, Bonplandia 5: 295, 297. 1857; Paul in Sharma et al., FI. India 3: 277. 1993; Maliya \& Datt, J. Econ. Taxon. Bot. 34 (1): 47. 2010.

Malva coromandeliana L., Sp. Pl. 687. 1753. Malvastrum tricuspidatum A.Gray., PI. Wright. 1: 16. 1852; Mast. in Hook. f., FI. Brit. India 1: 321. 1874; Duthie, Fl. Upp. Gang. Plain 1: 75. repr. ed. 1960.

Habit: Herb.

Specimens examined: 10.xi.2002, Murtiha range, coll. S.D. Maliya 224596 (LWG).

**Sida acuta Burm. f., Fl. Ind. 147. 1768; Duthie, Fl. Upp. Gang. Plain 1: 76. repr. ed. 1960; Paul in Sharma et al., Fl. India 3: 281. 1993; Panigrahi et al., Bull. Bot. Surv. India 11 (1 \& 2): 98. 1969; Maliya \& Datt, J. Econ. Taxon. Bot. 34 (1): 47. 2010.

S. carpinifolia L. f., Suppl. 307. 1781; Mast. in Hook. f., FI. Brit. India 1: 323. 1874.

Habit: Undershrub.

Specimens examined: 21.xi.1964, Nishangara range, G. Panigrahi \& O.P. Mishra 6500 (BSA); 11.xi.2002, Murtiha range, coll. S.D. Maliya 224599 (LWG).

Sida cordata (Burm. f.) Borss. Waalk., Blumea 14: 182. 1966; Panigrahi et al., Bull. Bot. Surv. India 11 (1 \& 2): 98. 1969; Paul in Sharma et al., Fl. India 3: 283. 1993; Maliya \& Datt, J. Econ. Taxon. Bot. 34 (1): 47. 2010.

Melochia cordata Burm. f., FI. Ind. 143. 1768. Sida veronicaefolia Lam., Encycl. 1: 5. 1783; Mast. in Hook. f., FI. Brit. India 1: 322. 1874; Duthie, FI. Upp. Gang. Plain 1: 75. repr. ed. 1960.

Habit: Herb.

Specimens examined: 26.v.1954, Motipur range, Hira Lal 13453 (LWG); 11.iii.1964, Motipur range, G. Panigrahi 2836 (BSA); 11.ii.1965, Bichhiya, O.P. Mishra 7918 (BSA); 07.x.2009, Kakraha range, coll. S.D. Maliya 226653 (LWG).

Sida cordifolia L., Sp. PI. 684. 1753; Mast. in Hook. f., Fl. Brit. India 1: 324. 1874; Duthie, Fl. Upp. Gang. Plain 1: 77. repr. ed. 1960; Panigrahi et al., Bull. Bot. Surv. India 11 (1 \& 2): 98. 1969; Paul in Sharma et al., FI. India 3: 285. 1993; Maliya \& Datt, J. Econ. Taxon. Bot. 34 (1): 47. 2010;
Saini, J. Econ. Taxon. Bot. 29 (3): 545. 2005.

Habit: Undershrub.

Specimens examined: 20.xi.1964, Nishangara range, G. Panigrahi \& O.P. Mishra 6438 (BSA); 09.xi.2002, near Bichhiya, Katerniaghat range, coll. S.D. Maliya 224509 (LWG); 12.xi.2002, Nishangara range, coll. S.D. Maliya 224635 (LWG); 26.xi.2002, Nishangara range, coll. S.D. Maliya 224641 (LWG).

**Sida rhombifolia L., Sp. Pl. 684. 1753; Mast. in Hook. f., Fl. Brit. India 1: 323. 1874; Panigrahi et al., Bull. Bot. Surv. India 11 (1 \& 2): 98. 1969; Paul in Sharma et al., FI. India 3: 289. 1993; Maliya \&Datt, J. Econ. Taxon. Bot. 34 (1): 47. 2010.

Habit: Undershrub.

Specimens examined: 22.xi.1964, Orla, Motipur range, G. Panigrahi \& O.P. Mishra 6585 (BSA); 26.viii.2002, road side, Katerniaghat range, coll. S.D. Maliya 223760 (LWG); 10.xi.2002, Murtiha range, coll. S.D. Maliya 224597 (LWG); 26.x.2004, Nepal border, Murtiha range, coll. S.D. Maliya 227120 (LWG).

Thespesia lampas (Cav.) Dalz. \& Gibs., Bombay Fl. 19. 1861; Paul in Sharma et al., Fl. India 3: 350. 1993; Maliya \& Datt, J. Econ. Taxon. Bot. 34 (1): 47. 2010.

Hibiscus lampas Cav., Diss. 3: 154, t. 56, f. 2. 1787.

Habit: Shrub or small tree.

Specimens examined: 12.xi.2002, Nishangara range, coll. S.D. Maliya 224643 (LWG); 30.viii.2003, Murtiha range, coll. S.D. Maliya 214864 (LWG).

Triumfetta pentandra A. Rich. in Guill. \& Perr., Fl. Seneg. Tent. 93, t. 19. 1831; Daniel \& Chandrabose in Sharma et al., Fl. India 3: 519. 1993; Maliya \& Datt, J. Econ. Taxon. Bot. 34 (1): 48. 2010.

T. neglecta Wight \& Arn., Prodr. 1: 75. 1834; Mast. in Hook. f., Fl. Brit. India 1: 396. 1874.

Habit: Herb.

Specimens examined: 23.viii.2002, Nishangara range, coll. S.D. Maliya 223650 (LWG); 12.xi.2002, Nishangara range, coll. S.D. Maliya 224637 (LWG).

Triumfetta rhomboidea Jacq., Enum. Syst. PI. 22. 1760; Mast. in Hook. f., Fl. Brit. India 1: 395. 1874; Duthie, Fl. Upp. Gang. Plain 1: 111. repr. ed. 1960; Daniel \& Chandrabose in Sharma et al., Fl. India 3: 520. 1993; Maliya \& Datt, J. Econ. Taxon. Bot. 34 (1): 48. 2010.

Habit: Herb or small shrub.

Specimens examined: 07.xii.1986, Motipur range, K.K. Khanna \& R. Saran 38574 (BSA); 17.v.1987, K.K. Singh \& party 5946 (LWG); 12.xi.2002, Nishangara range, coll. S.D. 
Maliya 224638 (LWG).

Urena lobata L., Sp. PI. 692. 1753; Mast. in Hook. f., FI. Brit. India 1: 329. 1874; Duthie, Fl. Upp. Gang. Plain 1: 80. repr. ed. 1960; Panigrahi et al., Bull. Bot. Surv. India 11 (1 \& 2): 98. 1969; Paul in Sharma et al., Fl. India 3: 380. 1993; Maliya \& Datt, J. Econ. Taxon. Bot. 34 (1): 48. 2010.

Habit: Shrub or undershrub.

Specimens examined: 26.v.1954, Motipur range, Hira Lal 13460 (LWG); 12.ii.1965, Rampurwa, O.P. Mishra 7948 (BSA); 07.xii.1986, Motipur range, K.K. Khanna \& R. Saran 38575 (BSA); 24.xi.1987, K.K. Singh \& party 6186 (LWG); 12.xi.2002, Nishangara range, coll. S.D. Maliya 224653 (LWG); 10.x.2009, road side, Katerniaghat range, coll. S.D. Maliya 226671 (LWG).

Urena repanda Roxb. ex Sm., Cycl. 37: Urena no. 6. 1819.

Pavonia repanda (Roxb. ex J. E. Sm.) Spreng., Syst. 3: 98. 1826; Walkes, Blumea 14: 135. 1966; Panigrahi et al., Bull. Bot. Surv. India 11 (1 \& 2): 98. 1969.

Habit: Herb.

Specimens examined: 20.xi.1964, Nishangara range, G. Panigrahi \& O.P. Mishra 6433 (BSA); 05.xi.2008, Katerniaghat range, coll. S.D. Maliya 226639 (LWG).

\section{MARTYNIACEAE}

Martynia annua L., Sp. Pl. 618. 1753; Panigrahi et al., Bull. Bot. Surv. India 11 (1 \& 2): 102. 1969.

M. diandra Glox, Observ. Bot. 14, t. 1. 1785.

Habit: Herb.

Specimens examined: 22.xi.1964, Orla, Motipur range, G. Panigrahi \& O.P. Mishra 6579 (BSA); 29.x.2004, Kuthiyaghat forest, coll. S.D. Maliya 227144 (LWG).

\section{MELIACEAE}

*Azadirachta indica A. Juss., Mem. Mus. Hist. Nat. 19: 221, t. 2, f. 5. 1830; Jain \& Bennet et al. in Hajra et al., Fl. India 4: 478. 1997; Maliya \& Datt, J. Econ. Taxon. Bot. 34 (1): 49. 2010.

Melia azadirchta L., Sp. PI. 385. 1753; Hiern. in Hook. f., Fl. Brit. India 1:544. 1875; Duthie, Fl. Upp. Gang. Plain 1: 141. repr. ed. 1960.

Habit: Tree, cultivated.

Specimens examined: 20.iv.2010, near railway crossing, Katerniaghat range, L.B. Chaudhary, A. Kumar \& O. Bajpai 252217 (LWG).

*Melia azadirach L., Sp. PI. 384. 1753; Hiern. in Hook. f., Fl. Brit. India 1: 544. 1875; Duthie, FI. Upp. Gang. Plain 1: 141. repr. ed. 1960; Jain \& Bennet et al. in Hajra et al.,
Fl. India 4: 494. 1997; Maliya \& Datt, J. Econ. Taxon. Bot. 34 (1): 49. 2010.

Habit: Tree, cultivated

Specimens examined: 17.iv.2003, on the bank of river, Katerniaghat range, coll. S.D. Maliya 224907 (LWG); 24.xii.2003, Kuthiyaghat forest, coll. S.D. Maliya 225252 (LWG); 21.iv.2010, Bichhiya chauk, Nishangara range, L.B. Chaudhary, A. Kumar \& O. Bajpai 252246 (LWG); 22.iv.2010, Infront of Mote baba temple, near urai nala, A. Kumar \& O. Bajpai 252263 (LWG).

Toona ciliata M. Roem., Fam. Nat. Syn. Monogr. 1: 139. 1846; Jain \& Bennet et al. in Hajra et al., Fl. India 4: 504. 1997.

T. hexandra M. Roem., Fam. Nat. Syn. Monogr. 1: 139. 1846; Maliya \& Datt, J. Econ. Taxon. Bot. 34 (1): 49.2010. Cedrella toona Roxb. ex Willd., Berl. Gesel. Nat. Fr. Neue Schr. 4: 198. 1803; Hiern. in Hook. f., Fl. Brit. India 1: 568. 1875; Duthie, Fl. Upp. Gang. Plain 1: 143. repr. ed. 1960.

Habit: Tree.

Specimens examined: 18.iv.2003, road side, Katerniaghat range, coll. S.D. Maliya 224932 (LWG); 28.viii.2003, Murtiha range, Katerniaghat wildlife anctuary, Bahraich, coll. S.D. Maliya 214854 (LWG); 31.viii.2003, Amba forest, coll. S.D. Maliya 214893 (LWG); 16.iii.2004, Karmohini forest, coll. S.D. Maliya 225285 (LWG); 19.ii.2009, Kakraha rest house, Kakraha range, L.B. Chaudhary, A. Kumar \& O. Bajpai 250254 (LWG); 20.iv.2010, near salarpur, A. Kumar \& O. Bajpai 252211

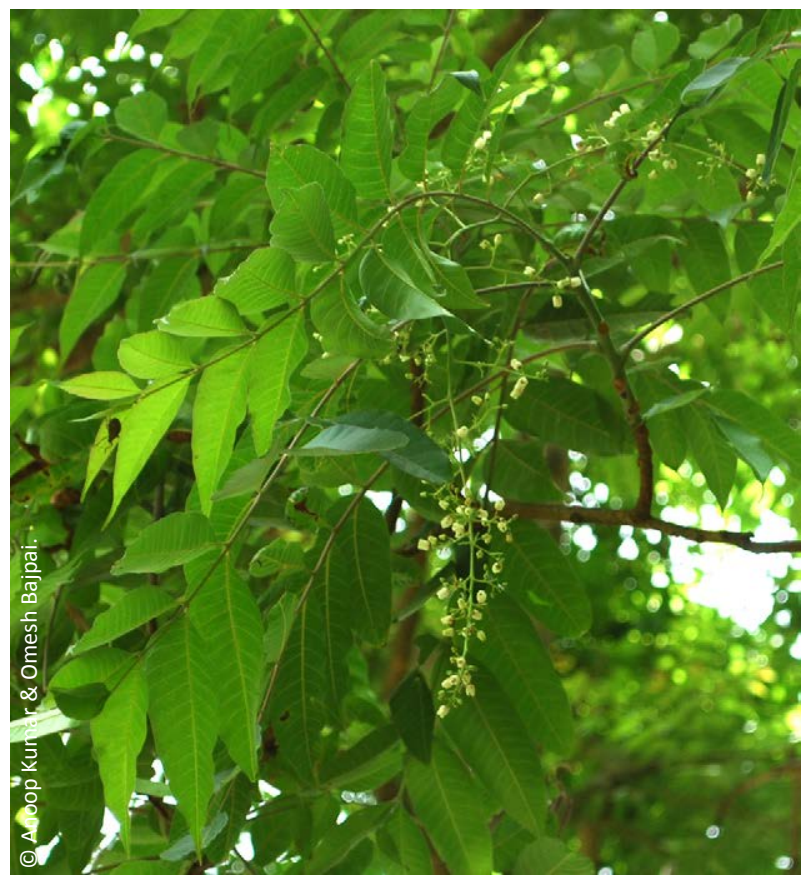

Image 43. Toona ciliata M. Roem. 
(LWG); 26.iv.2010, road side, Girijapuri forest, A. Kumar \& O. Bajpai 252288 (LWG); 04.iv.2011, Near Katerniaghat dam, A. Kumar, O. Bajpai \& K. Kishore 263622 (LWG) (Image 43).

Trichilia connaroides (Wight \& Arn.) Bentvelzen, Acta Bot. Neerl. 11: 13. 1962; Singh, Fl. Dudhwa National Park 120. 1997; Jain \& Bennet in Hajra et al., Fl. India 4: 515, f. 122. 1997.

Heynea trijuga Roxb. ex Sims, Bot. Mag. 42: t. 1738. 1815 (W. Roxburgh, Hort. Bengal. 33. 1814, nom. nud.). Zanthoxyllum connaroides Wight \& Arn., Prodr. FI. Ind. Orient. 1: 148. 1834.

Habit: Tree.

Specimens examined: 21.iv.2010, Nishangara range, A. Kumar \& O. Bajpai 252248 (LWG); 22.iv.2010, Near rampurwa rest house, Nishangara range, L.B. Chaudhary, A. Kumar \& O. Bajpai 252250 (LWG) (Image 44).

\section{MENISPERMACEAE}

**Cissampelos pareira L. var. hirsuta (Buch.-Ham. ex DC.) Forman, Kew Bull. 22: 356. 1968; Gangopadhyay in Sharma et al., Fl. India 1: 317. 1993; Maliya \& Datt, J. Econ. Taxon. Bot. 34 (1): 46. 2010.

C. hirsuta Buch.-Ham. ex DC., Syst. Nat.1: 535. 1818. C. pareira sensu Panigrahi et al., Bull. Bot. Surv. India 11 (1 \& 2): 102. 1969.

Habit: Twining shrub.

Specimens examined: 25.v.1987, Nishangara range, K.K. Singh \& party 5956 (LWG); 23.viii.2002, Nishangara range, coll. S.D. Maliya 223653 (LWG); 24.viii.2002, Nishangara range, coll. S.D. Maliya 223688 (LWG); 09.ix.2002, roadside, Katerniaghat range, coll. S.D. Maliya 224558 (LWG); 11.xi.2002, Murtiha range, coll. S.D. Maliya 224605 (LWG); 18.iii.2004, Kuthiyaghat forest, coll. S.D. Maliya 225907; 23.iv.2004, Katerniaghat range, Kayerniaghat wildlife sanctuary, Bahraich, coll. S.D. Maliya 225943 (LWG); 26.x.2009, Nishangara to Katerniaghat road, L.B. Chaudhary, A. Kumar \& O. Bajpai 252128 (LWG); 26.x.2009, Nishangara to Katerniaghat road, L.B. Chaudhary, A. Kumar \& O. Bajpai 252129 (LWG); 02.xi.2009, near petrol pump, Katerniaghat range, L.B. Chaudhary, A. Kumar \& O. Bajpai 252183 (LWG).

Tiliacora acuminata (Lam.) Miers, Ann. Mag. Nat. Hist. 7: 39. 1851; Hook. f. \& Thomson, Fl. Ind. 187. 1855; Panigrahi et al., Bull. Bot. Surv. India 11 (1 \& 2): 102. 1969; Pramanik in Sharma et al., FI. India 1: 343. 1993; Saini, J. Econ. Taxon. Bot. 29 (3): 536. 2005; Maliya \& Datt, J. Econ. Taxon. Bot. 34 (1): 46.2010.
Menispermum acuminatum Lam., Encycl. 4: 101. 1797. Tiliacora racemosa Colebr., Trans. Linn. Ser. 13: 67. 1821.

Habit: Climber.

Specimens examined: 26.v.1954, Motipur range, Hira Lal 13449 (LWG); 24.xi.1987, Rampurwa, K.K. Singh \& party 6184 (LWG); 23.viii.2002, Nishangara forest, K.K. Singh \& coll. S.D. Maliya 223651 (LWG); 23.viii.2002, Nishangara forest, K.K. Singh \& coll. S.D. Maliya 223603 (LWG); 25.viii.2002, Murtiha range, coll. S.D. Maliya 223732 (LWG); 27.xii.2003, mid point of Katerniaghat \& Nishangara, coll. S.D. Maliya 225273 (LWG); 04.vi.2009, Chafaria chauk, Nishangara range, L.B. Chaudhary, A. Kumar \& O. Bajpai 250626 (LWG).

Tinospora cordifolia (Willd.) Miers, Ann. Mag. Nat. Hist. 7: 38. 1851; Hook. f. \& Thomson, Fl. Ind. 1: 184. 1855 \& in Hook. f., Fl. Brit. India 1: 97. 1872; Duthie, Fl. Upp. Gang. Plain 1: 27. repr. ed. 1960; Pramanik in Sharma et al., Fl. India 1: 347. 1993; Saini, J. Econ. Taxon. Bot. 29 (3): 536. 2005.

Menispermum cordifolium Willd., Sp. PI. 4: 826. 1806.

Habit: Climbing shrub.

Specimens examined: Not observed. Record based on Saini (2005).

\section{MENYANTHACEAE}

Nymphoides indica (L.) Kuntze, Revis. Gen. Pl. 2: 429. 1891; Saini, J. Econ. Taxon. Bot. 29 (3): 617. 2005; Maliya \& Datt, J. Econ. Taxon. Bot. 34 (1): 57. 2010.

Menyanthes indica L., Sp. Pl. 145.1753. Limnanthemum indicum (L.) Griseb., Gen. Sp. Gent. 343. 1839.

Habit: Aquatic herb.

Specimens examined: 24.iv.2004, road side, Kailashpuri forest, coll. S.D. Maliya 227101 (LWG); 04.iii.2011, Babhnia Fata, Kakraha range, A. Kumar, O.

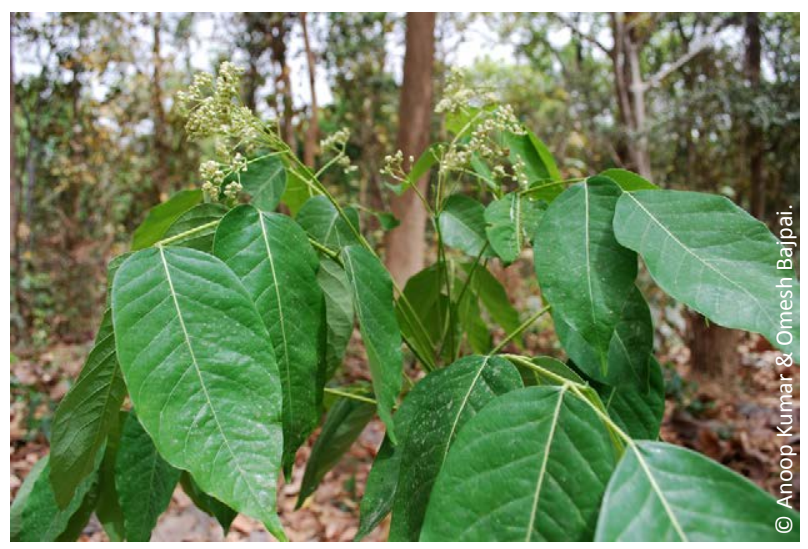

Image 44. Trichilia connaroides (Wight \& Arn.) Bentvelzen 
Bajpai \& K. Kishor 252846 (LWG).

\section{MolluginaceAe}

**Glinus lotoides L., Sp. PI. 463. 1753; Panigrahi et al., Bull. Bot. Surv. India 11 (1 \& 2): 103. 1969; Saini, J. Econ. Taxon. Bot. 29(3): 587. 2005.

Mollugo hirta Thunb., Prodr. Fl. Cap. 24. 1799; Clarke in Hook. f., Fl. Brit. India 2: 662. 1879. M. lotoides O. Kuntze, Rev. Gen. PI. 264. 1891.

Habit: Herb.

Specimens examined: 25.v.1954, Motipur range, Hira Lal 13434 (LWG); 10.xii.1986, Motipur range, K.K. Khanna \& R. Saran 37693 (BSA).

**Mollugo pentaphylla L., Sp. pl. 89. 1753

Habit: Herb.

Specimens examined: 11.iii.1964, Motipur range, G. Panigrahi 2835 (BSA).

\section{MoRACEAE}

*Artocarpus heterophyllus Lam., Encycl. 3: 209. 1789 "heterophylla"; Santapau, Bull. Bot. Surv. India 3: 12. 1962; Panigrahi et al., Bull. Bot. Surv. India 11 (1 \& 2): 96. 1969; Maliya \& Datt, J. Econ. Taxon. Bot. 34 (1): 63. 2010.

Habit: Tree, cultivated.

Specimens examined: 22.iv.2003, near ranger office, Nishangara range, coll. S.D. Maliya 224989 (LWG); 24.iv.2003, Murtiha range, coll. S.D. Maliya 214827 (LWG).

*Artocarpus lakoocha Roxb., FI. Ind. 3: 524. 1832; King in Hook. f., Fl. Brit. India 5: 543. 1888 pp.; Duthie, Fl. Upp. Gang. Plain 3: 234. repr. ed. 1960.

A. lacucha Buch.-Ham. in Mem. Wern. Nat. Hist. Soc. 5: 333.1826.

Habit: Tree, cultivated.

Specimens examined: 20.iv.2010, near Salarpur village, Murtiha range, L.B. Chaudhary, A. Kumar \& O. Bajpai, 252214 (LWG).

Broussonetia papyrifera (L.) L'H'er ex Vent., Tabl. Regn. Veg. 3: 547. 1799; Maliya \& Datt, J. Econ. Taxon. Bot. 34 (1): 63.2010.

Morus papyrifera L.,Sp. PI. 986. 1753.

Habit: Tree.

Specimens examined: 17.iv.2003, Katerniaghat range, coll. S.D. Maliya \& B. Datt 224901 (LWG) (Image 45).

Ficus assamica Miq. in Hook. London J. Bot. 7: 226. 1848; Kumar et al., American J. PI. Sci. 2: 80, f. 1. 2011;Kumar et al., Phtotaxonomy 12: 82-88.2012;
Chaudhary et al., Taiwania 57 (2): 200. 2012.

F. repens Roxb. ex Willd., Sp. PI. 4 (2): 1149. 1806 (non Rottl. 1803). F. heterophylla var. repens (Roxb. ex Willd.) King, Ann. Roy. Bot. Gard. Calcutta. 1: 77. 1888; Kanjilal, For. Fl. Pilibhit, Oudh, Gorakhpur \& Bundelkhand 365. 1933. F. heterophylla f. repens (Roxb. ex Willd.) King, Hook. f., Fl. Brit. India 5: 519. 1888. F. heterophylla var. assamica (Miq.) Corner, Gard. Bull. Singapore 21: 73. 1965.

Habit: Herb or undershrub.

Specimens examined: 08.vi.2009, near Vardia Chauki, Katiyara, L.B. Chaudhary, A. Kumar \& O. Bajpai 250601 (LWG); 18.vii.2009, L.B. Chaudhary, A. Kumar \& O. Bajpai 250811 (LWG); 21.ii.2009, L.B. Chaudhary, A. Kumar \& O. Bajpai 250812 (LWG).

Ficus benghalensis L., Sp. PI. 1059. 1753; King, Ann. Roy. Bot. Gard. Calcutta 1: 18, t. 13 \& 81c. 1887 \& in Hook. f., Fl. Brit. India 5: 499. 1888; Duthie, Fl. Upp. Gang. Pl. 3 (1): 147. 1915; Panigrahi et al., Bull. Bot. Surv. India 11 (1 \& 2): 96. 1969; Saini, J. Econ. Taxon. Bot. 29 (4): 878. 2005; Maliya \& Datt, J. Econ. Taxon. Bot. 34 (1): 63. 2010; Kumar et al., American J. PI. Sci. 2: 81, f. 2. 2011; Chaudhary et al., Taiwania 57 (2): 210. 2012.

F. indica L., Sp. Pl. 1060. 1753. non sensu King (1887). F. cotonaefolia Vahl, Enum. PI. 2: 189. 1806.

Habit: Tree.

Specimens examined: 12.xi.2002, Nishanghara forest, coll. S.D. Maliya 224660 (LWG); 24.iv.2003, Murtiha forest, coll. S.D. Maliya 214829 (LWG); 09.xii.2008, Rampurva rest house, L.B. Chaudhary \& A. Kumar 250111 (LWG); 22.ii.2009, Gangapur village, L.B. Chaudhary \& A. Kumar 250258 (LWG); 21.vii.2010, Near Kakraha rest house, L.B. Chaudhary, A. Kumar \& O. Bajpai 252810 (LWG).

*Ficus elastica Roxb. (Hort. Beng. 65. 1814, nom.

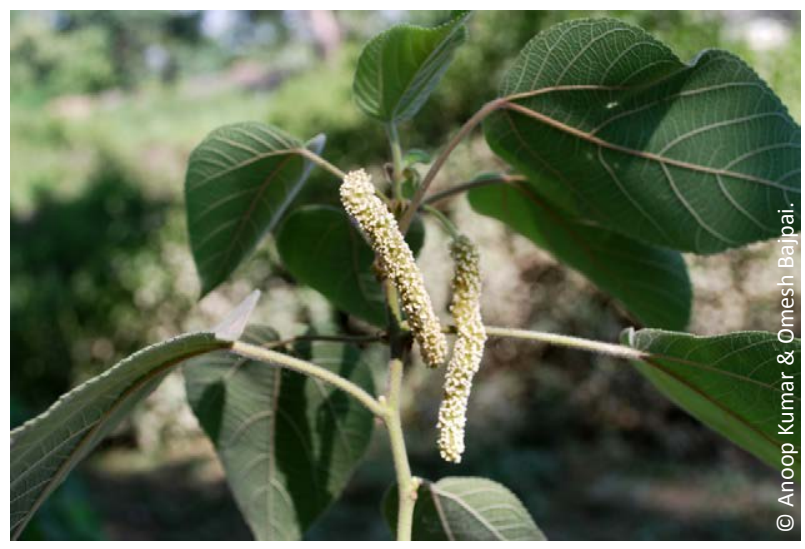

Image 45. Broussonetia papyrifera (L.) L’H’er ex Vent. 
nud.) ex Hornem., Hort. Bot. Hafn. Suppl. 7. 1819; Duthie, Fl. Upper Gangetic Plain 3 (1): 161. 1915; Chaudhary et al., Taiwania 57 (2): 212. 2012.

Habit: Tree, cultivated.

Specimens examined: 10.iii.2011. rest house, Kakraha range, A. Kumar \& O. Bajpai 252900 (LWG).

Ficus heterophylla L. f., Suppl. PI. 442. 1781; King, Ann. Roy. Bot. Gard. Calcutta 1: 75, t. 94. 1888 \& in Hook. f., Fl. Brit. India 5: 518. 1888; Duthie, Fl. Upp. Gang. PI. 3 (1): 154. 1915, pp. (excl. F. repens Willd.); Saini, J. Econ. Taxon. Bot. 29 (4): 878. 2005; Maliya \& Datt, J. Econ. Taxon. Bot. 34 (1): 63.2010 pp.; Kumar et al., American J. PI. Sci. 2: 82, f. 3. 2011; Chaudhary et al., Taiwania 57 (2): 201. 2012

Habit: Scandent shrub.

Specimens examined: 19.vii.2004, Kuthiyaghat forest, coll. S.D. Maliya 225979 (LWG); 08.vi.2009, near Vardia Chauki, L.B. Chaudhary, A. Kumar \& O. Bajpai 250629 (LWG);

Ficus hispida L. f., Suppl. PI. 442. 1782; King, Ann. Roy. Bot. Gard. Calcutta 1: 116, t. 154 - 155. 1888 \& in Hook. f., Fl. Brit. India 5: 522. 1888; Duthie, Fl. Upp. Gang. PI. 3 (1): 155. 1915; Panigrahi et al., Bull. Bot. Surv. India 11 (1 \& 2): 97. 1969; Saini, J. Econ. Taxon. Bot. 29 (4): 878. 2005; Maliya \& Datt, J. Econ. Taxon. Bot. 34 (1): 63. 2010; Kumar et al., American J. PI. Sci. 2: 83, f. 4. 2011; Chaudhary et al., Taiwania 57 (2): 205. 2012.

Habit: Tree.

Specimens examined: 23.viii.2002, Nishangara range, coll. S.D. Maliya \& B. Datt 223666 (LWG); 18.iv.2003, Bichhiya town, coll. S.D. Maliya 224929 (LWG); 22.iv.2003, Nishangara range, coll. S.D. Maliya 214818 (LWG); 27.viii.2003, Bichhiya town, coll. S.D. Maliya 214848 (LWG); 16.iii.2004, coll. S.D. Maliya 252288 (LWG); 23.xii.2005, Nishangara range, coll. S.D. Maliya 227180 (LWG); Karmohini forest, coll. S.D. Maliya 225288 (LWG); 22.ii. 2009, near Babhnia Fata village, L.B. Chaudhary, A. Kumar \& O. Bajpai 250267 (LWG); 08.iv.2009, Rampurva rest house, L.B. Chaudhary, A. Kumar \& O. Bajpai 250297 (LWG); 09.iv.2009, near Babhnia Fata village, L.B. Chaudhary, A. Kumar \& O. Bajpai 250289 (LWG); Near Kakraha rest house, 21.vii.2010, L.B. Chaudhary, A. Kumar \& O. Bajpai 252809 (LWG) (Image 46).

Ficus microcarpa L. f, Suppl. PI. 442. 1782; Panigrahi et al., Bull. Bot. Surv. India 11 (1 \& 2): 97. 1969; Chaudhary et al., Taiwania 57 (2): 212. 2012.

Habit: Tree.

Specimens examined: Not observed. Record based on
Panigrahi et al. (1969).

Ficus palmata Forssk. subsp. virgata (Roxb.) Browicz in Rechinger, Fl. Iranica 153. 12. 1982; Kumar et al., American J. PI. Sci. 2: 84, f. 5. 2011; Chaudhary et al., Taiwania 57 (2): 194. 2012.

F. virgata Roxb., Fl. Ind. 3: 530. 1832; Wight, Icon. PI. Ind. Orient. t. 649. 1841. F. palmata sensu King, Ann. Bot. Gard. Calcutta 1: 146, t. 185. 1888 pp. (excl. syn. F. pseudo-sycomorus Decne) \& in Hook. f., Fl. Brit. India 5: 530. 1888 pp. (excl. syn. F. pseudo-sycomorus Decne); Duthie, Fl. Upp. Gang. PI. 3 (1): 158. 1915; Panigrahi in Bull. Bot. Surv. India 11 (1 \& 2): 97. 1969; Maliya \& Datt in J. Econ. Taxon. Bot. 34 (1): 63. 2010. F. carica sensu Maliya \& Datt, J. Econ. Taxon. Bot. 34 (1): 63. 2010.

Habit: Shrub or small tree.

Specimens examined: 09.ii.1965, Jairampurwa, Motipur range, O.P. Mishra 7755 (BSA); 23.viii.2002, Nishangara forest, coll. S.D. Maliya 223662 (LWG); 16.iii.2004, Karmohini forest, coll. S.D. Maliya (LWG); 08.iv.2009, near Katerniaghat Barrier, L.B. Chaudhary 250294 (LWG); 24.iv.2010, Nishangarha range, near Bhainsa Lotan Fata village, L.B. Chaudhary, A. Kumar \& O. Bajpai 252270 (LWG).

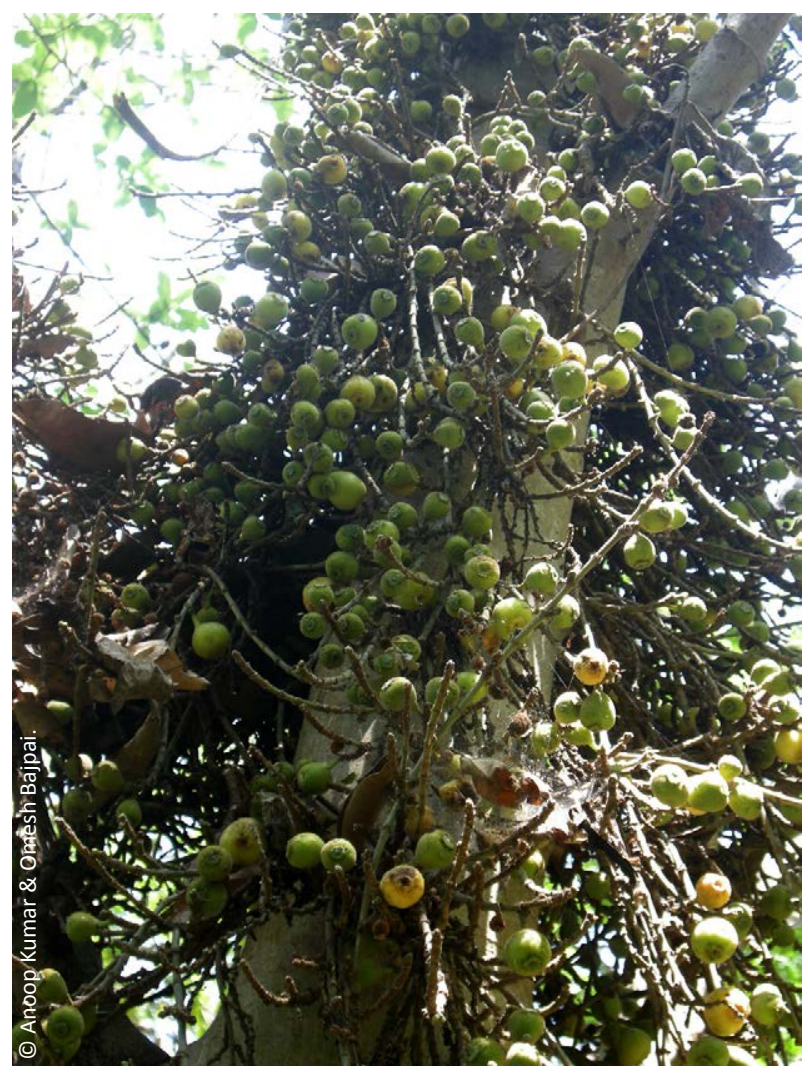

Image 46. Ficus hispida L. f. 
Ficus racemosa L., Sp. PI. 1060. 1753; King, Ann. Roy. Bot. Gard. Calcutta 1: 183. 1888; Panigrahi, Bull. Bot. Surv. India 11 (1 \& 2): 97. 1969; Saini, J. Econ. Taxon. Bot. 29 (4): 878. 2005; Maliya \& Datt, J. Econ. Taxon. Bot. 34 (1): 63. 2010; Kumar et al., American J. PI. Sci. 2: 85, f. 6. 2011; Chaudhary et al., Taiwania 57 (2): 203. 2012.

F. glomerata Roxb., PI. Coromandel 2: 13, t. 123. 1798; King in Ann. Roy. Bot. Gard. Calcutta 1: 173, t. 218. 1888 \& in Hook. f., Fl. Brit. India 5: 535. 1888; Duthie, Fl. Upp. Gang. PI. 3 (1): 160. 1915.

Habit: Tree.

Specimens examined: 19.ii.2009, rest house, Kakraha range, L.B. Chaudhary \& A. Kumar 250255 (LWG); 22.ii.2009, Babhnia Fata village, L.B. Chaudhary \& A. Kumar 250260 (LWG); 23.ii.2009, Semal chowk, Taratal piller no. 76, L.B. Chaudhary \& A. Kumar 250270 (LWG); 15.vi.2009, Near Geruva river, Katiyara road, L.B. Chaudhary, A. Kumar \& O. Bajpai 250608 (LWG); 21.vii.2010, Nishangarha range office, L.B. Chaudhary, A. Kumar \& O. Bajpai 252808 (LWG) (Image 47).

Ficus religiosa L., Sp. PI. 1059. 1753; King, Ann. Roy. Bot. Gard. Calcutta 1: 55, t. 67a. 1887 \& in Hook. f., FI. Brit. India 5: 513. 1888; Duthie, Fl. Upp. Gang. PI. 3 (1): 150. 1915; Saini, J. Econ. Taxon. Bot. 29 (4): 879. 2005; Maliya \& Datt, J. Econ. Taxon. Bot. 34 (1): 64. 2010; Kumar

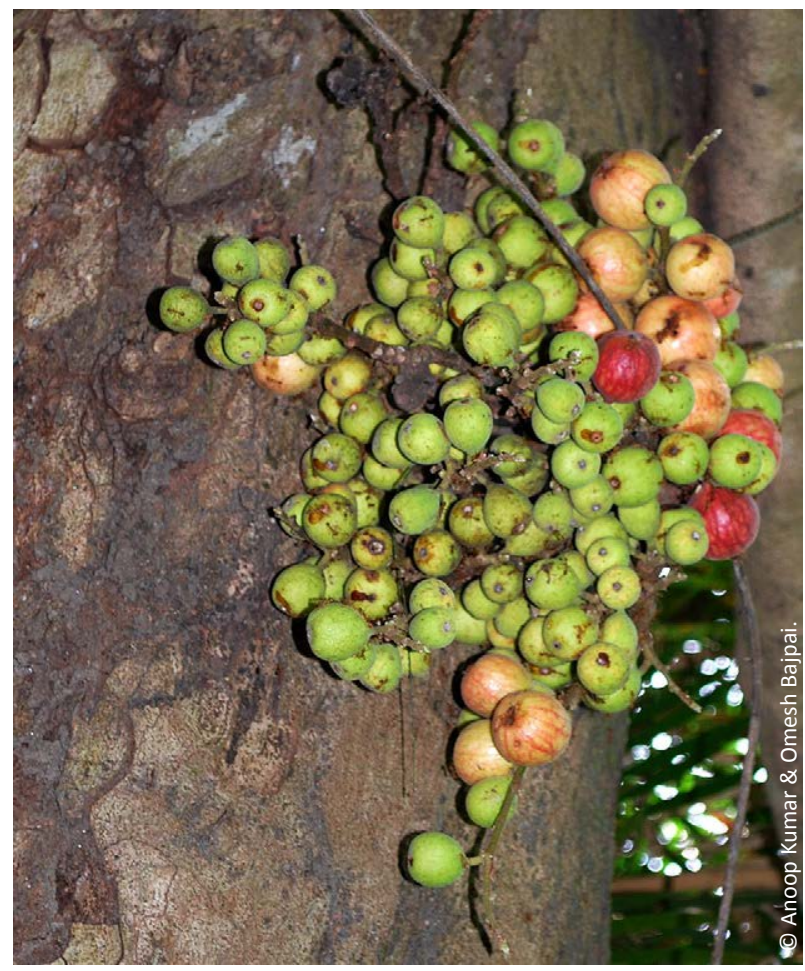

Image 47. Ficus racemosa L. et al., American J. PI. Sci. 2: 87, f. 7. 2011; Chaudhary et al., Taiwania 57 (2): 208. 2012.

Habit: Tree.

Specimens examined: 20.v.1990, Dharampur, Motipur range, K.K. Khanna 63 (BSA); 18.iv.2003, Bichhiya town, coll. S.D. Maliya 224933 (LWG); 24.x.2009, Kakraha rest house, L.B. Chaudhary, A. Kumar \& O. Bajpai 252107 (LWG); 21.vii.2010, rest house, Kakraha range, L.B. Chaudhary, A. Kumar \& O. Bajpai 252807 (LWG).

**Ficus retusa var. nitida (Thunb.) Miq., Ann. Mus. Bot. Lugd.-Bat. 3: 267. 1867; King, Ann. Roy. Bot. Gard. Calcutta 1: 51, t. 62, 84P. 7 - 9. 1887; Duthie, Fl. Upp. Gang. PI. 3 (1): 149. 1915; Kumar et al., American J. PI. Sci. 2: 88, f. 8. 2011; Chaudhary et al., Taiwania 57 (2): 213. 2012.

F. nitida Thunb., Ficus 10. 1786; Wight, Icon. PI. Ind. Orient. 2: t. 642. 1843. Urostigma nitidum (Thunb.) Miq. in Hook. London J. Bot. 6: 582. 1847. Ficus retusa sensu Maliya \& Datt, J. Econ. Taxon. Bot. 35 (1): 36. 2011. F. retusa sensu Maliya, J. Econ. Taxon. Bot. 36 (2): 423.2012.

Habit: Tree.

Specimens examined: 19.ii.2009, behind Kakraha rest house, L.B. Chaudhary, A. Kumar \& O. Bajpai 250257 (LWG); 10.x.2009, rest house, Kakraha range, coll. S.D. Maliya 226678 (LWG); 24.x.2009, behind Kakraha rest house, L.B. Chaudhary, A. Kumar \& O. Bajpai 252104 (LWG).

Ficus rumphii Bl., Bijdr. Fl. Ned. Ind. 437. 1825; King, Ann. Roy. Bot. Gard. Calcutta 1: 54, t. 67b \& 84t. 1887 \& in Hook. f., Fl. Brit. India 3: 512. 1888; Duthie, Fl. Upp. Gang. PI. 3 (1): 151. 1915; Srivastava, Fl. Gorakhpurensis 307. 1976; Berg \& Corner, Fl. Malesiana 17 (2): 609. 2005; Kumar et al., American J. PI. Sci. 2: 89, f. 9. 2011; Chaudhary et al., Taiwania 57 (2): 209. 2012.

F. cordifolia Roxb., Fl. Ind. 3: 548. 1832 (non BI. 1825). F. conciliorum (Rumph.) Oken. in Allg. Naturgesch. 3: 1561. 1841. F. populnea Kunth \& Bouche, Ind. Sem. Hort. Berol. 16. 1846. Urostigma cordifolium (Roxb.) Miq., Hook. London J. Bot. 5: 564. 1847. U. rumphii (BI.) Miq., Zoll. Syst. Verz. 2: 90. 1854. Ficus populiformis Schott. ex Miq., Ann. Mus. Bot. Lugd. - Bat. 3: 287. 1867.

Habit: Tree.

Specimens examined: 24.x.2009, rest house, Kakraha range, L.B. Chaudhary, A. Kumar \& O. Bajpai 252106 (LWG); 22.x.2010, rest house, Kakraha range, O. Bajpai 252818 (LWG).

Ficus semicordata Buch.-Ham. ex J. E. Sm. in Rees Cyclop. 14. Ficus n. 71. 1810; Saini, J. Econ. Taxon. Bot. 


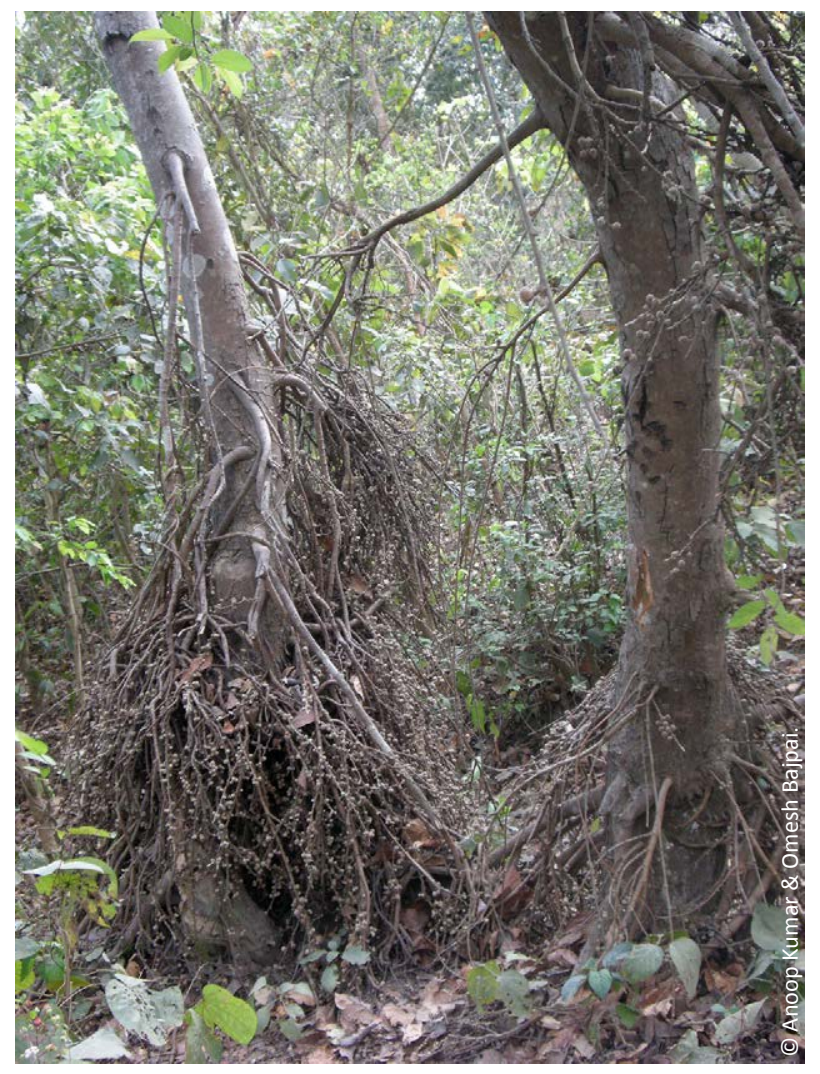

Image 48. Ficus semicordata Buch.-Ham. ex J. E. Sm.

29 (4): 879. 2005; Maliya \& Datt, J. Econ. Taxon. Bot. 34 (1): 64. 2010; Kumar et al., American J. PI. Sci. 2: 90, f. 10. 2011; Chaudhary et al., Taiwania 57 (2): 204. 2012.

F. cunia Buch. - Ham. ex Roxb., Fl. Ind. 3: 561. 1832; King, Ann. Roy. Bot. Gard. Calcutta 1: 101, t. 126. 1888 \& in Hook. f., Fl. Brit. India 5: 523. 1888 pp. (excl. F. conglomerata Roxb. 1832); Duthie, Fl. Upp. Gang. PI. 3 (1): 156. 1915.

Habit: Tree.

Specimens examined: 14.iv.1967, S. L. Kapoor \& party 70042 (LWG); 08.xii.1986, Katerniaghat range, K.K. Khanna \& R. Saran 37602 (BSA); 16.v.1987, K.K. Singh \& party 5965 (LWG); 09.iv.2009, near Balcha Barrier, Kakraha range, L.B. Chaudhary 250290 (LWG); 09.iv.2009, near Murtiha barrier, L.B. Chaudhary 250291 (LWG); 31.x.2009, Sujauli road, near Oripurva village, L.B. Chaudhary, A. Kumar \& O. Bajpai 252173 (LWG); 31.x.2009, Sujauli road, near Oripurva Village, L.B. Chaudhary, A. Kumar \& O. Bajpai 252174 (LWG) (Image 48).

Ficus squamosa Roxb., FI. Ind. 3: 531. 1832; Wu et al. in Wu et al., Fl. China 5: 49. 2003; Kumar et al., American J. PI. Sci. 2: 91, f. 11. 2011; Chaudhary et al., Taiwania 57 (2): 204. 2012.
F. pyrrhocarpa Kurz, Fl. Brit. Burm. 2: 457. 1832. F. saemocarpa Miq., Ann. Mus. Bot. Lugd. - Bat. 3: 32. 1867; King, Ann. Roy. Bot. Gard. Calcutta 1: 115, t. 152. 1888 \& in Hook. f., Fl. Brit. India 5: 523. 1888. F. laminosa Hardw. ex Roxb., Fl. Ind. 3: 531. 1832; Duthie, Fl. Upp. Gang. PI. 3 (1): 156. 1915.

Habit: Shrub or small tree.

Specimens examined: behind rest house, Kakraha range, 22. 10. 2009, L.B. Chaudhary, A. Kumar \& O. Bajpai 250700 (LWG); 22.x.2010, near Girijapuripuri petrol pump, O. Bajpai 252819 (LWG).

*Morus alba L., Sp. PI. 986. 1753; Hook. f. in Hook. f., Fl. Brit. India 5: 492. 1888; Duthie, Fl. Upp. Gang. Plain 3: 231. repr. ed. 1960; Maliya \& Datt, J. Econ. Taxon. Bot. 34 (1): 64. 2010.

Habit: Tree, cultivated.

Specimens examined: 20.iii.2004, Girijapuri forest, coll. S.D. Maliya 225932 (LWG); 28.x.2004, Nishangara range, coll. S.D. Maliya 227133 (LWG).

Streblus asper Lour., Fl. Cochinch. 2: 615. 1790; Hook. f. in Hook. f., Fl. Brit. India 5: 489. 1888; Duthie, Fl. Upp. Gang. Plain 3: 184. repr. ed. 1960; Maliya \& Datt, J. Econ. Taxon. Bot. 34 (1): 64. 2010.

Diplothorax tonkinensis Gagnep., Bull. Soc. Bot. France 75: 98-99. 1928.

Habit: Tree.

Specimens examined: 22.iv.2003, Nishangara range, coll. S.D. Maliya 224985 (LWG); 22.iv.2003, Nishangara range, coll. S.D. Maliya 224995 (LWG); 23.iii.2004, Kuthiyaghat forest, coll. S.D. Maliya 225937(LWG) (Image 49).

\section{MORINGACEAE}

*Moringa concanensis Nimmo ex Dalz. \& Gibb., Bomb. Fl. 311. 1861; Hook. f. in Hook. f., Fl. Brit. India 2: 45. 1879; Saini, J. Econ. Taxon. Bot. 29 (3): 557. 2005.

Habit: Tree, cultivated.

Specimens examined: Not observed. Record based on Saini (2005).

*Moringa oleifera Lam., Encycl. 1: 398. 1785; Maliya \& Datt, J. Econ. Taxon. Bot. 34 (1): 50. 2010.

M. pterigosperma Gaertn., Fruct. Sem. PI. 2: 314, t. 147, f. 2. 1791; Hook. f. in Hook. f., Fl. Brit. India 2: 45. 1879.

Habit: Tree, cultivated.

Specimens examined: 18.iv.2003, Bicchiya town, coll. S.D. Maliya 224926 (LWG); 10.ii.2010, near Amba village, L.B. Chaudhary, A. Kumar \& O. Bajapi 252193 (LWG). 


\section{MYRTACEAE}

*Callistemon citrinus (Curtis) Skeels, Bull. Bur. PI. Industr. U. S. D. A. 282: 49. 1913.

Metrosideros citrinus Curtis, Bot. Mag. 8: t. 260. 1794.

Callistemon lanceolatus DC., Prodr. 3: 223. 1828.

Habit: Tree, cultivated.

Specimens examined: 26.iv.2010, Sujauli road, L.B. Chaudhary, A. Kumar \& O. Bajpai 252292 (LWG).

*Eucalyptus tereticornis Sm., Spec. Bot. New Holland 41. 1795; Maliya \& Datt, J. Econ. Taxon. Bot. 34 (1): 52. 2010.

Habit: Tree, cultivated.

Specimens examined: 23.iv.2003, Murtiha range, coll. S.D. Maliya 214821 (LWG).

*Psidium guajava L., Sp. Pl. 470. 1753; Duthie in Hook. f., Fl. Brit. India 2: 468. 1879.

Habit: Tree, cultivated.

Specimens examined: 17.v.1987, Katerniaghat range, K.K. Singh \& party 5953 (LWG); 21.iv.2010, near Bichhia railway station, Katerniaghat Wildlife Sanctuary, L.B. Chaudhary, A. Kumar \& O. Bajpai 252232 (LWG).

Syzygium cumini (L.) Skeels, Bull. Bur. Pl. Industr. U.S.D.A. 248: 25. 1912; Panigrahi et al., Bull. Bot. Surv. India 11 (1 \& 2): 99. 1969; Maliya \& Datt, J. Econ. Taxon. Bot. 34 (1): 52. 2010.

Myrtus cumini L., Sp. PI. 471. 1753. Eugenia jambolana Lam., Encycl. 3: 198. 1789; Duthie in Hook. f., Fl. Brit. India 2: 499.1879.

Habit: Tree.

Specimens examined: 20.xi.1964, Uraital, Nishangara range, G. Panigtrahi \& O.P. Mishra 6465 (BSA); 21.v.1987, Katerniaghat range, K.K. singh \& party 5987 (LWG); 20.iv.2003, Murtiha range, coll. S.D. Maliya 224946 (LWG); 09.iv.2009, road side, Kakraha range, L.B. Chaudhary, A. Kumar \& O. Bajpai 252206 (LWG) (Image 50).

Syzygium heyneanum (Duthie) Gamble, Fl. Madras 3: 482. 1919; Saini, J. Econ. Taxon. Bot. 29 (3): 578. 2005.

Eugenia heyneana Duthie in Hook. f., Fl. Brit. India 2: 500. 1879.

Habit: Tree.

Specimens examined: Not observed. Record based on Saini (2005)

Syzygium nervosum A. Cunn. ex DC., Prodr. 3: 260. 1828.

Eugenia operculata Roxb., Fl. Ind. 2: 486. 1825; Duthie in Hook. f., Fl. Brit. India 2: 498. 1879. E. cerasoides Roxb.,
Fl. Ind. 2: 488. 1832. Syzygium cerasoides (Roxb.) Raizada, Indian Forester 84: 478. 1958; Chatterjee \& Kanjilal f., J. Bombay Nat. Hist. Soc. 55: 520. 1958 \& For. Fl. Plains U. P. 2: 331. 1966; Saini, J. Econ. Taxon. Bot. 29 (3): 578. 2005.

Habit: Tree.

Specimens examined: 25.iv.2010, behind rest house, Kakraha range, L.B. Chaudhary, A. Kumar \& O. Bajpai 252275 (LWG) (Image 51).

\section{Nelumbonaceae}

Nelumbo nucifera Gaertn., Fruct. 1: 73, t. 19, f. 2. 1788; Panigrahi et al., Bull. Bot. Surv. India 11 (1 \& 2): 102. 1969; Mitra in Sharma et al., FI. India 1: 441. 1993; Saini, J. Econ. Taxon. Bot. 29 (3): 537. 2005; Maliya \& Datt , J. Econ. Taxon. Bot. 34 (1): 46. 2010.

Nelumbium speciosum Willd., Sp. Pl. 2: 1258. 1799; Hook. f. \& Thoms. in Hook. f., Fl. Brit. India 1: 116. 1782.

Habit: Aquatic herb.

Specimens examined: 24.xi.1964, G. Panigrahi \& O.P. Mishra 6610 (BSA); 20.vii.2004, Kailashpuri forest, coll. S.D. Maliya 225972 (LWG).

\section{NYCTAGINACEAE}

Boerhaavia diffusa L., Sp. PI. 3. 1753; Duthie, Fl. Upp. Gang. Plain 3: 127. repr. ed. 1960; Panigrahi et al., Bull. Bot. Surv. India 11 (1 \& 2): 97. 1969; Maliya \& Datt, J. Econ. Taxon. Bot. 34 (1): 61. 2010.

Habit: Herb.

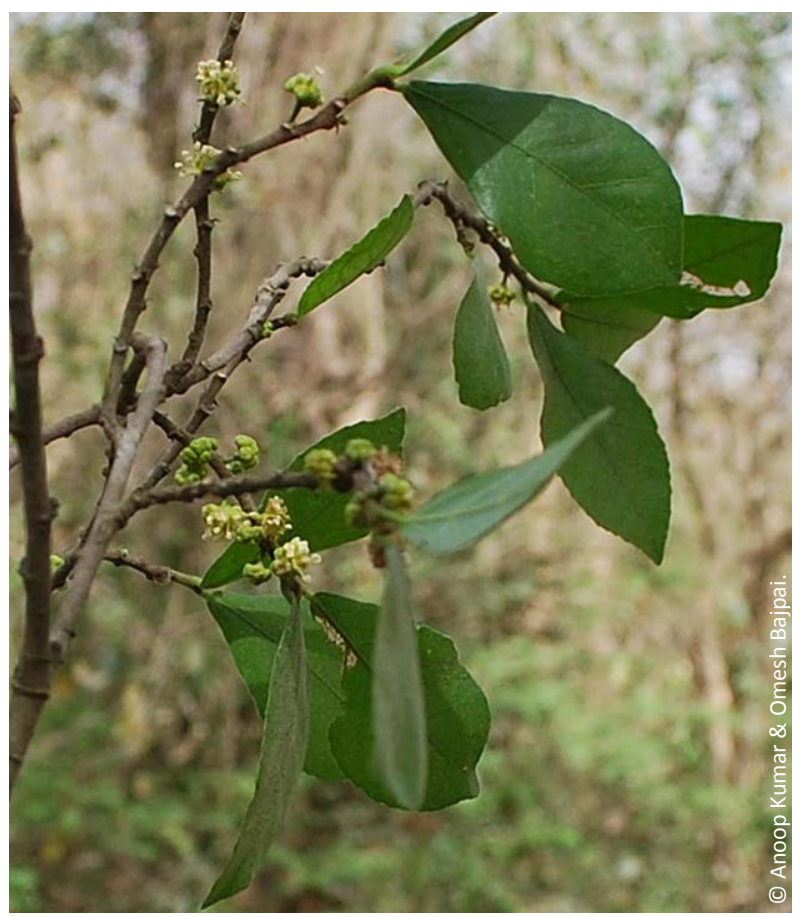

Image 49. Streblus asper Lour. 
Specimens examined: 25.v.1954, Motipur range, Hira Lal 13416 (LWG); 17.v.1987, Katerniaghat Wildlife Sanctuary, Baharich, K.K. Singh \& party 5955; 24.viii.2002, Nishangara range, coll. S.D. Maliya 223681 (LWG); 25.viii.2002, Murtiha range, coll. S.D. Maliya 223716 (LWG); 11.xi.2002, Murtiha range, coll. S.D. Maliya 224600 (LWG); 31.viii.2003, Fakirpur village, Nishangara range, coll. S.D. Maliya 214872 (LWG).

\section{NYMPHAEACEAE}

Nymphaea nouchali Burm. f., Fl. Ind. 120. 1768; Mitra in Sharma et al., Fl. India 1: 430. 1993; Saini, J. Econ.

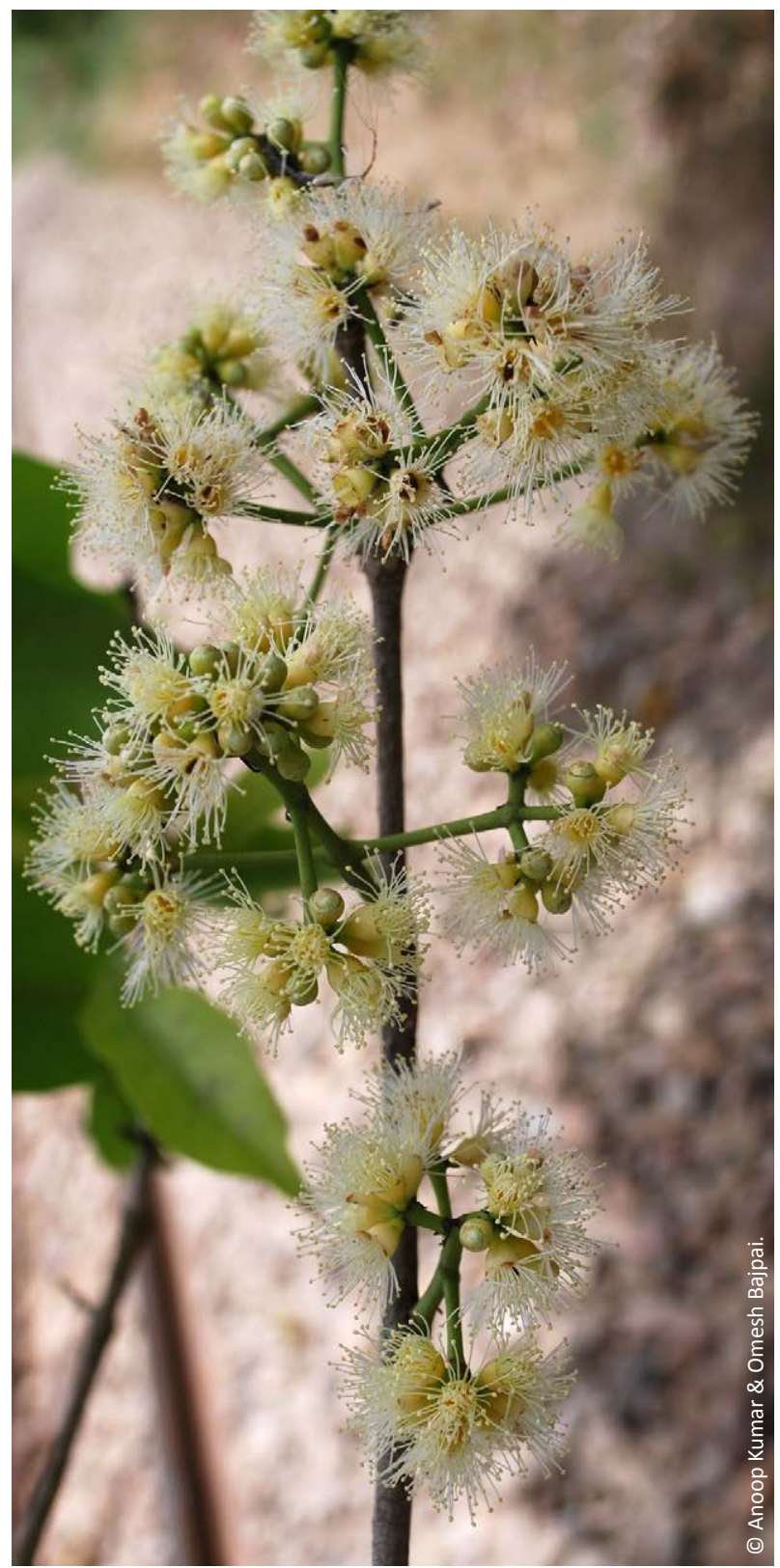

Image 50. Syzygium cumini (L.) Skeels
Taxon. Bot. 29 (3): 536. 2005.

N. stellata Willd., Sp. PI. ed. 4, 2: 1154. 1799; Hook. f. \& Thoms. in Hook. f., Fl. Brit. India1: 114. 1872; Duthie, Fl. Upp. Gang. Plain 1: 34. repr. ed. 1960.

Habit: Aquatic herb.

Specimens examined: Not observed. Record based on Saini (2005).

Nymphaea pubescens Willd., Sp. PI. 2: 1154. 1799; Mitra in Sharma et al., Fl. India 1: 431. 1993.

Nymphaea nouchali sensu Maliya \& Datt, J. Econ. Taxon. Bot. 34 (1): 46. 2010.

Habit: Aquatic herb.

Specimens examined: 22.x.2004, Kuthiyaghat forest, coll. S.D. Maliya 225989 (LWG).

\section{OLACACEAE}

**Olax nana Wall. ex Benth., Proc. Linn. Soc. 1: 88. 1840 \& Trans. L. Soc. Bot. 18 (4): 678. 1841; Mast. in Hook. f., Fl. Brit. India 1: 576. 1875; Saini, J. Econ. Taxon. Bot. 29 (3): 553. 2005.

Habit: Undershrub.

Specimens examined: Not observed. Record based on Saini (2005).

Olax zeylanica L., Sp. PI. 34. 1753; Mast. in Hook. f., Fl. Brit. India 1: 576. 1875; Saini, J. Econ. Taxon. Bot. 29 (3): 553. 2005.

Habit: Shrub or small tree.

Specimens examined: Not observed. Record based on Saini (2005).

\section{Oleaceae}

*Jasminum auriculatum Vahl, Symb. Bot. 3: 1. 1794; Clarke in Hook. f., Fl. Brit. India 3: 600. 1882; Saini, J. Econ. Taxon. Bot. 29 (3): 608. 2005.

Habit: Bushy or climbing shrub, cultivated.

Specimens examined: Not observed. Record based on Saini (2005).

*Jasminum multiflorum (Burm. f.) Andrews, Bot. Repos. 8: t. 496. 1807; Saini, J. Econ. Taxon. Bot. 29 (3): 609. 2005; Maliya \& Datt, J. Econ. Taxon. Bot. 34 (1): 56. 2010.

Nyctanthes multiflora Burm. f., FI. Ind. 5, t. 3, f. 1. 1768. Jasminum pubescens (Retz.) Willd., Sp. PI. 37. 1797; Duthie, Fl. Upp. Gang. Plain 1: 475. repr. ed. 1960.

Habit: Scandent shrub, cultivated.

Specimens examined: 24.iv.2003, rest house, Murtiha gate, coll. S.D. Maliya 214831 (BSA). 


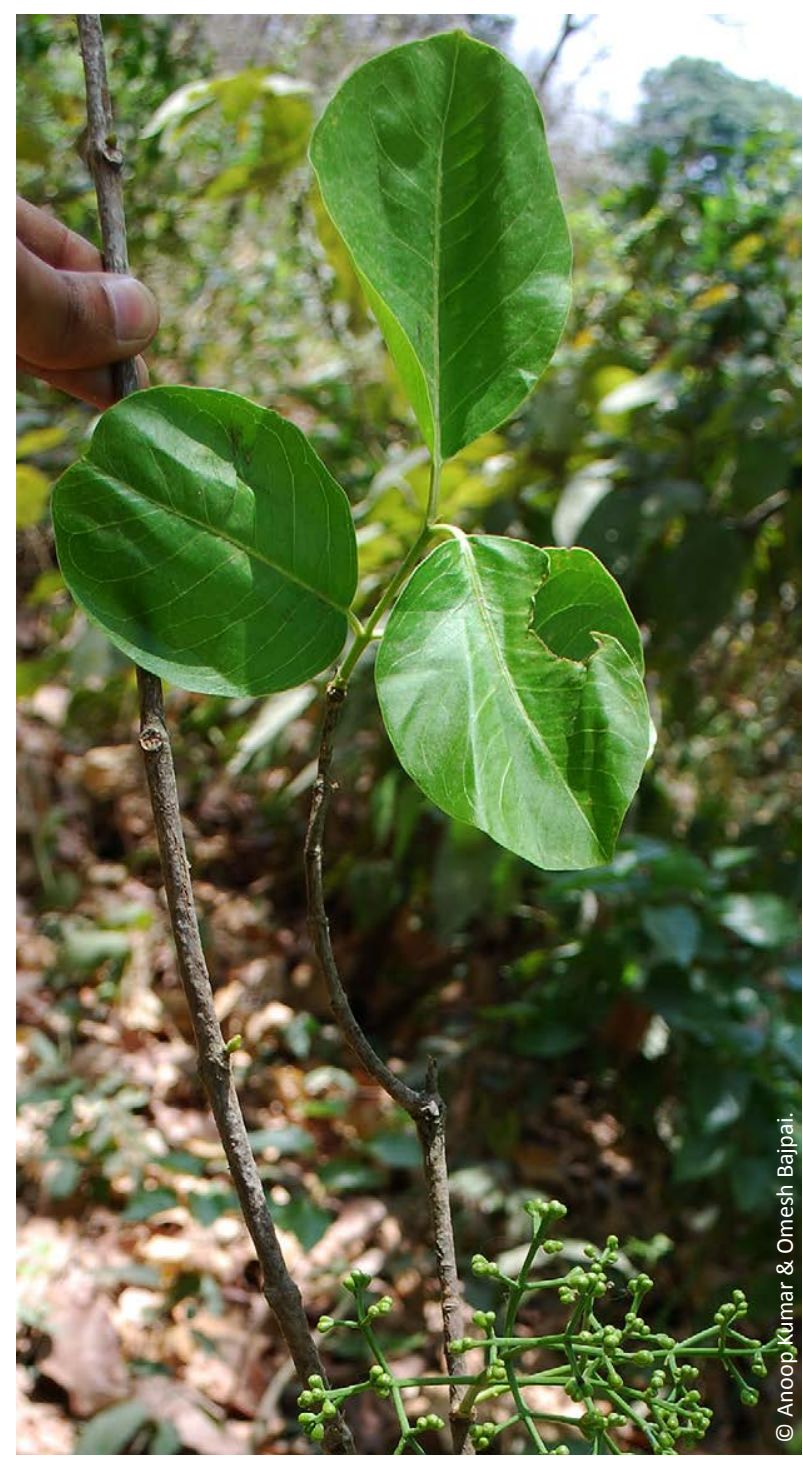

Image 51. Syzygium nervosum A. Cunn. ex DC.

*Nyctanthes arbor-tritis L., Sp. PI. 6. 1753; Roxb., Fl. Ind. 1: 85. 1832; Clarke in Hook. f., Fl. Brit. India 3: 603. 1882; Duthie, Fl. Upp. Gang. Plain 1: 477. repr. ed. 1960; Panigrahi et al., Bull. Bot. Surv. India 11 (1 \& 2): 101. 1969; Maliya \& Datt, J. Econ. Taxon. Bot. 34 (1): 56. 2010.

Habit: Shrub or small tree, cultivated.

Specimens examined: 12.iii.1964, Murtiha gate, G. Panigrahi 2855 (BSA); 10.xii.1986, Motipur range, Katerniaghat Wildlife Sanctuary, K.K. Khanna \& R. Saran 37700 (BSA); 28.xii.2003, near railway station, coll. S.D. Maliya 225279 (LWG); 31.x.2009, Murtiha range, A. Kumar \& O. Bajpai 252165 (LWG).

\section{ONAGRACEA}

Ludwigia adscendens (L.) Hara, J. Jap. Bot. 28: 291. 1953; Raven, Reinwardtia 6 (4): 387. 1963; Saini, J. Econ.
Taxon. Bot. 29 (3): 581. 2005.

Jussiaea adscendens L., Mant. PI. 1: 69. 1767. J. repens L., Sp. PI. 388. 1753. non Forst. 1771; Clarke in Hook. f., Fl. Brit. India 2: 587. 1879.

Habit: Aquatic herb.

Specimens examined: 27.v.1954, Motipur range, Hira Lal 13472 (LWG).

Ludwigia octovalis subsp. sessiliflora (Micheli) Raven, Kew Bull. 15(3): 476. 1962; Saini, J. Econ. Taxon. Bot. 29 (3): 581.2005.

Jussiaea octonervia var. sessiliflora Micheli in Martius, FI. Brass. 13: 180. 1875. J. suffrticosa L., Sp. PI. 388. 1753; Clarke in Hook. f., Fl. Brit. India 2: 587. 1879.

Habit: Herb.

Specimens examined: Not observed. Record based on Saini (2005).

Ludwigia perennis L., Sp. PI. 119. 1753; Maliya \& Datt, J. Econ. Taxon. Bot. 34 (1): 53. 2010.

L. parviflora Roxb., Hort. Bengal. 11. 1814; Fl. Ind. 1: 440. 1820; Clarke in Hook. f., Fl. Brit. India 2: 588. 1879; Duthie, Fl. Upp. Gang. Plain 1: 327. repr. ed. 1960.

Habit: Herb.

Specimens examined: 26.x.2004, Nepal border, Murtiha range, coll. S.D. Maliya 227118 (LWG); 07.x.2009, rest house, Kakraha range, coll. S.D. Maliya 226655 (LWG).

Ludwigia prostrata Roxb., Fl. Ind., 1: 441. 1820; Maliya, J. Econ. Taxon. Bot. 36 (1): 157. 2012 \& 36 (2): 421. 2012.

Habit: Herb.

Specimens examined: 04.xi.2008, Murtiha range, coll. S.D. Maliya 226623 (LWG).

\section{OXALIDACEAE}

*Averrhoa carambola L., Sp. PI. 428. 1753; Edg. \& Hook. f. in Hook. f., Fl. Brit. India 1: 439. 1874.

Habit: Tree, cultivated.

Specimens examined: 25.iv.2010, Nidhipurwa, Khalla village, Kakraha range, 252281 (LWG).

Biophytum sensitivum (L.) DC., Prodr. 1: 690. 1824; Edg. \& Hook. f. in Hook. f., Fl. Brit. India 1: 436. 1874; Panigrahi et al., Bull. Bot. Surv. India 11 (1 \& 2): 108. 1969; Manna in Sharma et al., FI. India 4: 258. 1997; Maliya \& Datt, J. Econ. Taxon. Bot. 34 (1): 48. 2010.

Oxalis sensitiva L., Sp. PI. 434. 1753.

Habit: Herb.

Specimens examined: 08.xii.1986, Nishangara range, K.K. Khanna \& R. Saran 37615 (BSA); 09.xi.2002, near 
Bichhiya railway track, Katerniaghat range, coll. S.D. Maliya 224522 (LWG); near Balcha barrier, Kakraha range, L.B. Chaudhary, A. Kumar \& O. Bajapi (LWG); 06.xi.2011, Savana grassland, Katerniaghat range, A. Kumar, O. Bajpai \& L.B. Chaudhary 263668 (LWG).

Oxalis corniculata L., Sp. PI. 435. 1753; Edg. \& Hook. f., Fl. Brit. India 1: 436. 1874; Panigrahi et al., Bull. Bot. Surv. India 11 (1 \& 2): 108. 1969; Manna in Sharma et al., Fl. India 4: 242. 1997; Maliya \& Datt, J. Econ. Taxon. Bot. 34 (1): 48. 2010.

Habit: Herb.

Specimens examined: 09.xi.2002, rest house, Katerniaghat range, coll. S.D. Maliya 224530 (LWG).

Oxalis dehradunensis Raiz., Suppl. Fl. Upp. Gang. Plain 37. 1976; Manna in Sharma et al., Fl. India 4: 246. 1997.

O. intermedia A. Rich., Hist. Phys. Cuba, PI. Vasc. 315. 1841; Saini, J. Econ. Taxon. Bot. 29 (3): 550. 2005.

Habit: Herb.

Specimens examined: Not observed. Record based on Saini.

\section{PAPAVERACEAE}

**Argemone mexicana L., Sp. PI. 508. 1753; Hook. f. \& Thoms. in Hook. f., Fl. Brit. India1: 117. 1872; Duthie, Fl. Upp. Gang. Plain 1: 37. repr. ed. 1960. Panigrahi et al., Bull. Bot. Surv. India 11 (1 \& 2): 103. 1969; Debnath \& Nayar in Sharma et al., Fl. India 2: 2. 1993; Saini, J. Econ. Taxon. Bot. 29 (3): 537: 2005; Maliya \& Datt, J. Econ. Taxon. Bot. 34 (1): 46. 2010.

Habit: Herb or undershrub.

Specimens examined: 11.03.1964, Motipur, G. Panigrahi 2838; 17.iv.2003, Katerniaghat range, coll. S.D. Maliya 224912 (LWG); 17.iii.2004, Kuthiyaghat forest, coll. S.D. Maliya 225901 (LWG).

Fumaria indica (Hausskn.) Pugsley, J. Linn. Soc. Bot. 44: 313. 1919; Panigrahi et al., Bull. Bot. Surv. India 11 (1 \& 2): 103. 1969; Ellis \& Balakrishnan in Sharma et al., FI. India 2: 84. 1993; Maliya \& Datt, J. Econ. Taxon. Bot. 34 (1): 46. 2010.

F. parviflora subsp. vaillantii (Lois.) Hook. f. in Hook. f., Fl. Brit. India 1: 128. 1872 pp; Duthie, Fl. Upp. Gang. Plain 1: 37. repr. ed. 1960. F. vaillantii var. indica Hausskn., Flora 56: 443. 1873.

Habit: Herb.

Specimens examined: 28.xii.2003, Katerniaghat range, coll. S.D. Maliya 225281 (LWG).

\section{Pedaliaceae}

*Sesamum indicum L., Sp. Pl. 634. 1753, nom. cons; Clarke in Hook. f., Fl. Brit. India 4: 387. 1884; Duthie, Fl. Upp. Gang. Plain 2: 47. repr. ed. 1960.

S. orientale L., Sp. PI. 634. 1753, nom. rej.; Maliya \& Datt, J. Econ. Taxon. Bot. 34 (1): 59. 2010. S. mulayanum N.

C. Nair, Bull. Bot. Surv. India 5: 252. 1964.

Habit: Herb, cultivated, occasionally found in forest.

Specimens examined: 23.viii.2002, Nishangara range, coll. S.D. Maliya 223644 (LWG); 25.viii.2002, Murtiha range, coll. S.D. Maliya 223730 (LWG); 10.x.2009, road side, Katerniaghat range, coll. S.D. Maliya 226672 (LWG).

\section{Phrymaceae}

Mazus pumilus (Burm. f.) Steenis, Nova Guinea 9: 31. 1958; Maliya, J. Econ. Taxon. Bot. 36 (2): 422. 2012.

Lobelia pumila Burm. f., FI. Indica 186, PI. 60, f. 3. 1768. Mazus rugosus Lour., Fl. Cochinch. 2: 385. 1790.

Habit: Herb.

Specimens examined: 10.xi.2002, Murtiha range, coll. S.D. Maliya 224575 (LWG); 13.xi.2002, Nishangara range, coll. S.D. Maliya 224662 (LWG); 21.xii.2003, Katerniaghat range, coll. S.D. Maliya 225226 (LWG); 22.ii.2005, Kuthiyaghat forest, coll. S.D. Maliya 224684 (LWG).

Mazus pumilus var. delavayi (Bonati) Chin ex Hong, Novon 6(4): 374. 1996.

M. delavayi Bonati, Bull. Herb. Boiss. 8: 530. 1980; Raizada in Suppl. Fl. Upp. Gang. Plain 188. 1976; Saini, J. Econ. Taxon. Bot. 29 (3): 633. 2005.

Habit: Herb.

Specimens examined: Not observed. Record based on Saini (2005).

\section{Phyllanthaceae}

**Antidesma ghaesembilla Gaertn., Fruct. Sem. PI. 1: 189, t. 39, f. 5. 1788; Hook. f. in Hook. f., Fl. Brit. India 5: 357. 1887; Duthie, Fl. Upp. Gang. Plain 3: 203. repr. ed. 1960; Maliya \& Datt, J. Econ. Taxon. Bot. 34 (1): 62.2010.

A. pubescens Roxb., Pl. Coromandel 2: 35, t. 167. 1802. A. panicualtum BI., Bijdr. 1128. 1827.

Habit: Tree.

Specimens examined: 20.vii.2004, Murtiha range, coll. S.D. Maliya 225983 (LWG).

Breynia vitis-idaea (Burm. f.) C.E.C. Fisch., Bull. Misc. Inform. Kew 1932 (2): 65. 1932.

Rhamnus vitis-idaea Burm. f., Fl. Ind. 61. 1768. Melanthesa rhamnoides (Retz.) Bl., Bijdr. 591. 1826; Panigrahi et al., Bull. Bot. Surv. India 11 (1 \& 2): 99. 1969. Breynia rhamnoides (Retz.) Muell - Arg., Prodr. 15 
(2): 440. 1866; Hook. f. in Hook. f., Fl. Brit. India 5: 330. 1887; Duthie, Fl. Upp. Gang. Plain 3: 197. repr. ed. 1960. Phyllanthus rhamnoides Retz., Observ. Bot. 5: 30. 1788. Habit: Shrub or small tree.

Specimens examined: 22.xi.1964, Orla, Murtiha range, G. Panigrahi \& O.P. Mishra 6533 (BSA).

Bridelia retusa (L.) A. Juss., Euphorb. Gen. 109. 1824; Duthie, Fl. Upp. Gang. Plain 3: 191. repr. ed. 1960; Panigrahi et al., Bull. Bot. Surv. India 11 (1 \& 2): 98. 1969; Maliya \& Datt, J. Econ. Taxon. Bot. 34 (1): 62. 2010.

Clutia retusa L., Sp. PI. 1042. 1753. C. squamosa Lam., Encycl. 2: 54. 1786. Bridelia squamosa (Lam.) Gehrm., Bot. Jahrb. Syst. 41 (95): 30. 1908; Maliya \& Datt, J. Econ. Taxon. Bot. 34 (1): 62.2010.

Habit: Tree.

Specimens examined: 15.iv.1967, S. L. Kapoor \& party 70049 (LWG); 26.xi.1987, K.K. Singh 6193 (LWG); 26.viii.2002, Katerniaghat range, K.K. Singh \& coll. S.D. Maliya 223779 (LWG); 09.xi.2002, road side, Katerniaghat range, K.K. Singh \& coll. S.D. Maliya 224534 (LWG); 09.xi.2002, road side, Katerniaghat range, coll. S.D. Maliya 224551 (LWG); 11.xi.2002, Murtiha range, coll. S.D. Maliya 224620 (LWG); 12.xi.2002, Nishangara range, coll. S.D. Maliya 224628 (LWG); 18.iv.2003, Bichhiya, Katerniaghat range, coll. S.D. Maliya 224928 (LWG); 21.xii.2003, road side, Katerniaghat range, coll. S.D. Maliya 225215 (LWG); 21.xii.2003, Katerniaghat range, coll. S.D. Maliya 225223 (LWG); 05.xi.2008, coll. S.D. Maliya 226640 (LWG); 10.x.2009, road side, Katerniaghat range, coll. S.D. Maliya 226670 (LWG).

Bridelia stipularis (L.) Bl., Bijdr. 597. 1826; Hook. f. in Hook. f., Fl. Brit. India 5: 270. 1887; Duthie, Fl. Upp. Gang. Plain 3: 192. repr. ed. 1960; Panigrahi et al., Bull. Bot. Surv. India 11 (1 \& 2): 99. 1969; Maliya \& Datt, J. Econ. Taxon. Bot. 34 (1): 62. 2010.

Clutia stipularis L., Mant. PI. 1: 127. 1767.

Habit: Climber.

Specimens examined: 14.iv.1967, S. L. Kapoor \& party 70044 (LWG); 22.iv.2003, Nishangara range, coll. S.D. Maliya 224980 (LWG).

**Glochidion daltoni (Mull. Arg.) Kurz, Forest Fl. Burma 2: 344. 1877; Panigrahi et al., Bull. Bot. Surv. India 11 (1 \& 2): 99.1969.

Phyllanthus daltonii Mull. Arg., Flora 48: 388. 1865.

Habit: Tree.

Specimens examined: 21.xi.1964, Nishangara range, G. Panigrahi \& O.P. Mishra 6526 (BSA).
Phyllanthus emblica L., Sp. PI. 982. 1753; Hook. f. in Hook. f., Fl. Brit. India 5: 289. 1887; Duthie, Fl. Upp. Gang. Plain 3: 199. repr. ed. 1960; Maliya \& Datt, J. Econ. Taxon. Bot. 34 (1): 62. 2010.

Emblica officinalis Gaertn., Fruct. Sem. PI. 2: 122, t. 108, f. 2. 1790; Panigrahi et al., Bull. Bot. Surv. India 11 (1 \& 2): 99.1969.

Habit: Tree.

Specimens examined: 16.v.1987, K.K. Singh \& party 5963 (LWG); 30.08.2003, Murtiha range, coll. S.D. Maliya 214869 (LWG) (Image 52).

Phyllanthus fraternus Webster, Contrib. Gray Herb. 176: 53. 1955 \& J. Ann. Arb. 38: 309, t. 19, f. G - H. 1957; Chaudhary \& Rao, Phytotaxonomy 2: 151, f. 1C, 4. 2002; Maliya \& Datt, J. Econ. Taxon. Bot. 34 (1): 62. 2010.

P. asperulatus Hutch., Bull. Misc. Inform. Kew 1920: 27. 1920; Panigrahi et al., Bull. Bot. Surv. India 11 (1 \& 2): 99. 1969; Chaudhary \& Rao, Phytotaxonomy 2: 143. 2002. Habit: Herb.

Specimens examined: 29.viii.2003, rest house, Nishangara range, coll. S.D. Maliya 214858 (LWG).

Phyllanthus reticulatus Poir., Encycl. 5: 298. 1804; Duthie, Fl. Upp. Gang. Plain 3: 199. repr. ed. 1960; Maliya \& Datt, J. Econ. Taxon. Bot. 34 (1): 63. 2010.

Kirganelia reticulata (Poir.) Baill., Etude Euphorb. 613. 1858; Saini, J. Econ. Taxon. Bot. 29 (4): 873. 2005.

Habit: Scandent shrub.

Specimens examined: 02.ix.2003, Kuthiyaghat forest, coll. S.D. Maliya 225210 (LWG); 25.xii.2003, Midpoint of Katerniaghat and Nishangara range, coll. S.D. Maliya 225268 (LWG).

Phyllanthus urinaria L., Sp. PI. 982. 1753; Hook. f. in Hook. f., Fl. Brit. India 5: 293. 1887; Duthie, Fl. Upp.

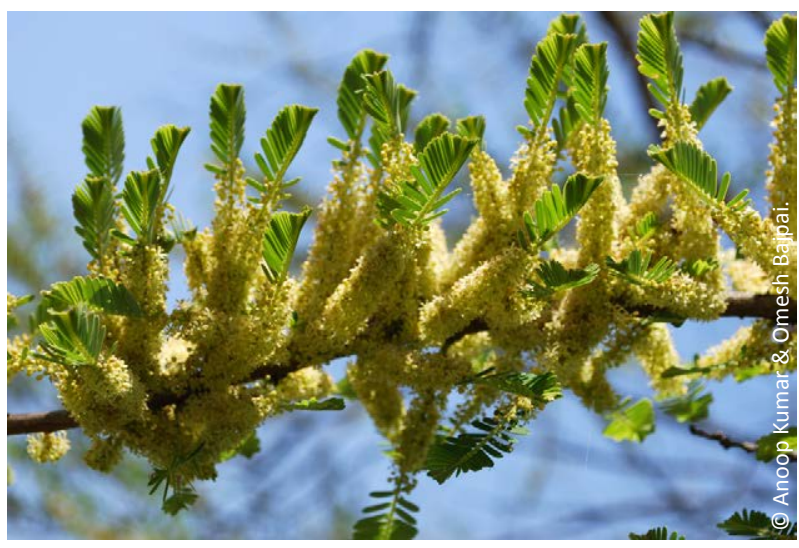

Image 52. Phyllanthus emblica L. 
Gang. Plain 3: 200. repr. ed. 1960; Chaudhary \& Rao, Phytotaxonomy 2: 158, f. 2C, 3. 2002; Saini, J. Econ. Taxon. Bot. 29(4): 875. 2005; Maliya \& Datt, J. Econ. Taxon. Bot. 34 (1): 63.2010.

P. leprocarpus Wight., Icon. PI. Ind. Orient. 5, t. 1895. 1852.

Habit: Herb.

Specimens examined: 23.viii.2002, Nishangara range, coll. S.D. Maliya 223631 (LWG); 25.viii.2002, rest house, Murtiha range, coll. S.D. Maliya 223746 (LWG); 26.viii.2002, Katerniaghat range, coll. S.D. Maliya 223772 (LWG); 09.xi.2002, near Bichhiya town, Katerniaghat range, coll. S.D. Maliya 224506 (LWG); 29.viii.2003, Nishangara range, coll. S.D. Maliya 214857 (LWG); 02.ix.2003, Kuthiyaghat forest, coll. S.D. Maliya 225202 (LWG); 19.07.2009, Kakraha range, L.B. Chaudhary, A. Kumar \& O. Bajpai 250654 (LWG).

Phyllanthus virgatus G. Forst., Fl. Ins. Austr. 65. 1786; Maliya \& Datt, J. Econ. Taxon. Bot. 34 (1): 63. 2010.

P. simplex Retz., Observ. Bot. 5: 29. 1789; Hook. f. in Hook. f., Fl. Brit. India 5: 295. 1887; Duthie, Fl. Upp. Gang. Plain 3: 200. repr. ed. 1960.

Habit: Herb or undershrub.

Specimens examined: Not observed. Record based on Maliya \& Datt (2010).

\section{Piperaceae}

Peperomia pellucida (L.) Kunth, Nov. Gen. Sp. 1: 64. 1815; Maliya \& Datt, J. Econ. Taxon. Bot. 34 (1): 62. 2010. Piper pellucidum L., Sp. PI. 30. 1753.

Habit: Herb.

Specimens examined: 25.viii.2002, rest house, Murtiha range, coll. S.D. Maliya 223748 (LWG); 09.xi.2002, rest house, Katerniaghat range, coll. S.D. Maliya 224527 (LWG); 26.x.2004, Nepal border, Murtiha range, coll. S.D. Maliya 227121 (LWG); 23.x.2010, Girijapuri forest, coll. S.D. Maliya 252629 (LWG).

Piper longum L., Sp. PI. 29. 1753; Hook. f. in Hook. f., Fl. Brit. India 5: 83. 1886; Raizada, Suppl. Fl. Upp. Gang. Plain 242. 1976; Panigrahi et al., Bull. Bot. Surv. India 11 (1 \& 2): 103. 1969; Maliya \& Datt, J. Econ. Taxon. Bot. 34 (1): 62.2010.

Habit: Creeping or rambling perennial herb.

Specimens examined: 23.viii.2002, Nishangara range, coll. S.D. Maliya 223652 (LWG); 25.viii.2002, Murtiha range, coll. S.D. Maliya 223742 (LWG); 09.xi.2002, near Bichhiya town, coll. S.D. Maliya 224521 (LWG); 14.xi.2002, rest house, Murtiha range, coll. S.D. Maliya 224675 (LWG); 07.ix.2005, Kailashpuri forest, coll. S.D.
Maliya 227155 (LWG).

\section{Plantaginaceae}

Bacopa monnieri (L.) Wettst., Nat. Pflanzenfam. 4 (3b): 77. 1891; Panigrahi et al., Bull. Bot. Surv. India 11 (1 \& 2): 106. 1969; Maliya \& Datt, J. Econ. Taxon. Bot. 34 (1): 58. 2010.

Lysimachia monnieri L., Cent. Pl. 2: 9. 1756. Bramia monnieri (L.) Pennell, Proc. Acad. Sci. Philadelphia 71(6): 243. 1920.

Note: Similar combination has been made by Pennell (Proc. Acad. Nat. Sci. Philadelphia 98 (2): 94. 1946).

Habit: Herb

Specimens examined: 27.v.1954, Motipur range, Hira Lal 13471 (LWG); 12.iii.1964, Motipur range, G. Panigrahi 2850 (BSA); 22.iv.2003, Nishangara range, coll. S.D. Maliya 224992 (LWG); 27.ii.2009, Haryue village, coll. S.D. Maliya 214909 (LWG).

Scoparia dulcis L., Sp. Pl. 116. 1753; Hook. f. in Hook. f., Fl. Brit. India 4: 289. 1884; Duthie, Fl. Upp. Gang. Plain 2: 27. repr. ed. 1960; Panigrahi et al., Bull. Bot. Surv. India 11 (1 \& 2): 107. 1969; Maliya \& Datt, J. Econ. Taxon. Bot. 34 (1): 59. 2010.

Habit: Undershrub.

Specimens examined: 26.v.1954, Motipur range, Hira Lal 13439 (LWG); 23.viii.2002, Nishangara range, coll. S.D. Maliya 223645 (LWG); 25.viii.2002, rest house, Murtiha range, coll. S.D. Maliya 223752 (LWG); 09.xi.2002, road side, Katerniaghat range, coll. S.D. Maliya 224543 (LWG).

**Veronica polita Fr., Fl. Novit. Suec. Alt. 1. 1828.

V. didyma Ten., Fl. Napol. 1:6. 1811; Maliya, J. Econ. Taxon. Bot. 36 (1): 159. 2012 \& 36 (2): 422.2012.

Habit: Herb.

Specimens examined: 22.ii.2005, Kuthiyaghat forest, coll. S.D. Maliya 224682 (LWG).

\section{Plumbaginaceae}

Plumbago zeylanica L., Sp. PI. 151. 1753.

Habit: Subscandent herb.

Specimens examined: 24.xi.1987, Nishangara range, K.K. Singh \& party 6174 (LWG); 04.xi.2011, near Rampurwa rest house, Nishangara range, A. Kumar, 0. Bajpai \& L.B. Chaudhary 263685 (LWG) (Image 53).

\section{Polygalaceae}

Polygala arvensis Willd., Sp. Pl. 3 (2): 876. 1803; Banerjee in Sharma et al., FI. India 2: 460. 1993; Maliya \& Datt, J. Econ. Taxon. Bot. 34 (1): 47. 2010.

Habit: Herb 
Specimens examined: 23.viii.2002, Nishangara range, coll. S.D. Maliya 223638 (LWG).

\section{Polygonaceae}

*Antigonon leptopus Hook. \& Arn., Bot. Beechey Voy. 308, t. 69. 1838; Maliya \& Datt, J. Econ. Taxon. Bot. 34 (1): 61. 2010.

Habit: Climbing shrub, cultivated.

Specimens examined: 27.x.2004, Katerniaghat range, coll. S.D. Maliya 227127 (LWG).

*Fagopyrum esculentum Moench, Methodus 290. 1794; Duthie, Fl. Upp. Gang. Plain 3: 159. repr. ed. 1960.

Polygonum fagopyrum L., Sp. PI. 364. 1753; Saini, J. Econ. Taxon. Bot. 29 (4): 865. 2005.

Habit: Herb, cultivated.

Specimens examined: Not observed. Record based on Saini (2005)

Persicaria barbata (L.) Hara, Fl. E. Himalaya 1: 70. 1966.

Polygonum barbatum L., Sp. PI. 362. 1753; Hook. f. in Hook. f., Fl. Brit. India 5: 37. 1886; Duthie, Fl. Upp. Gang. Plain 3: 154. repr. ed. 1960; Panigrahi et al., Bull. Bot. Surv. India 11 (1 \& 2): 103. 1969.

Habit: Herb.

Specimens examined: 24.xi.1964, Plantation, Katerniaghat range, G. Panigrahi \& O.P. Mishra 6609A (BSA); 09.ii.1965, Jairampurwa, O.P. Mishra 7762 (BSA).

Persicaria barbata (L.) Hara var. gracilis (Danser) Hara in Hara et al. Enum. Fl. PI. Nepal 175. 1982; Maliya \& Datt, J. Econ. Taxon. Bot. 34 (1): 61. 2010.

Polygonum barbatum L. ssp. gracilis Danser, Bull. Jard.

B. Buitenzorg Ser. 3, 8: 146. f. 2. 1927.

Habit: Herb.

Specimens examined: 10.xi.2002, Murtiha range, coll. S.D. Maliya 224578 (LWG).

**Persicaria lanigera (R. Br.) Sojak, Preslia 46: 153. 1974.

Polygonum lanigerum R. Br., Prodr. Fl. Nov. Holl. 419. 1810; Hook. f. in Hook. f., Fl. Brit. India 5: 35. 1886; Duthie, Fl. Upp. Gang. Plain 3: 152. repr. ed. 1960; Panigrahi et al., Bull. Bot. Surv. India 11 (1 \& 2): 103. 1969.

Habit: Herb.

Specimens examined: 21.xi.1964, Nishangara range, G. Panigrahi \& O.P. Mishra 6605 (BSA).

**Persicaria minor (Huds.) Opiz, Seznam 72. 1852.

Polygonum minus Huds., Fl. Augl. (ed.1): 148. 1762;
Hook. f. in Hook. f., Fl. Brit. India 5: 36. 1886; Raizada, Suppl. Fl. Upp. Gang. Plain 239. 1976; Saini, J. Econ. Taxon. Bot. 29 (4): 866. 2005.

Habit: Herb.

Specimens examined: Not observed. Record based on Saini (2005).

Persicaria serrulata (Lag.) Webb \& Moq., Hist. Nat. Iles Canaries 3: 219. 1846.

Polygonum serrulatum Lag., Gen. Sp. Pl. 14. 1816; Duthie, Fl. Upp. Gang. Plain 3: 155. repr. ed. 1960; Panigrahi et al., Bull. Bot. Surv. India 11 (1 \& 2): 103. 1969.

Habit: Herb.

Specimens examined: 26.v.1954, Motipur range, Hira Lal 13427 (LWG); 22.xi.1964, Motipur on the way to Orla, G. Panigrahi \& O.P. Mishra 6547 (BSA); 31.viii.2003, Fakirpur village, Nishangara range, coll. S.D. Maliya 214881 (LWG).

Persicaria stagnina (Buch.-Ham. ex Meisn.) Qaiser, Fl. Pakistan 205: 44. 2001.

Polygonum stagninum Buch. - Ham. ex Meissn., Fl. Asiat. Rar. 3: 56. 1832; Panigrahi et al., Bull. Bot. Surv. India 11 (1 \& 2): 103. 1969.

Habit: Herb.

Specimens examined: 20.xi.1964, Nishangara range, G. Panigrahi \& O.P. Mishra 6463 (BSA); 24.xi.1964, Nishangara range, G. Panigrahi \& O.P. Mishra 6607A (BSA); 14.ii.1965, Urai tal, Bichhiya, O.P. Mishra 7988 (BSA).

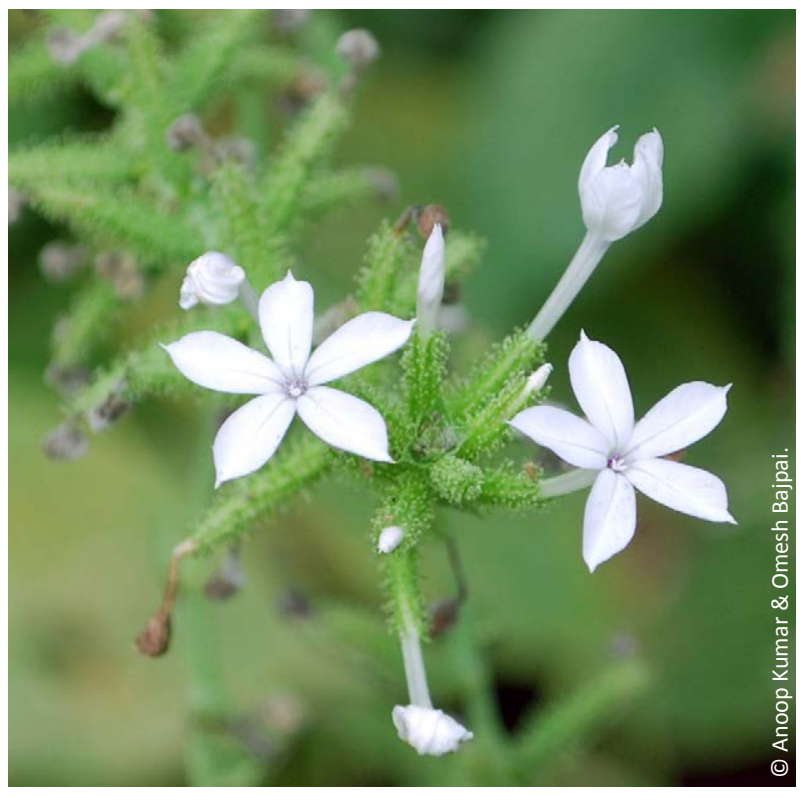

Image 53. Plumbago zeylanica L. 
Polygonum plebeium R. Br., Prodr. Fl. Nov. Holl. 420. 1810; Panigrahi et al., Bull. Bot. Surv. India 11 (1 \& 2): 103. 1969; Saini, J. Econ. Taxon. Bot. 29 (4): 866. 2005; Maliya \& Datt, J. Econ. Taxon. Bot. 34 (1): 62.2010.

Habit: Herb.

Specimens examined: 08.ii.1963, Motipur range, O.P. Mishra 7727 (BSA); 11.iii.1964, Sujauli, G. Panigrahi 2797 (BSA); 16.iii.2004, Karmohini forest, Katerniaghat range, coll. S.D. Maliya 225291 (LWG); 23.iii.2004, Kuthiyaghat forest, coll. S.D. Maliya 225942 (LWG).

Rumex dentatus L., Mant. PI. 2: 226. 1771; Hook. f. in Hook. f., Fl. Brit. India 5: 59. 1886; Duthie, Fl. Upp. Gang. Plain 3: 158. repr. ed. 1960; Panigrahi et al., Bull. Bot. Surv. India 11 (1 \& 2): 103. 1969.

Habit: Herb.

Specimens examined: 09.ii.1965, Jairampurwa, Motipur range, O.P. Mishra 7757 (BSA).

\section{PRIMULACEAE}

**Anagallis arvensis L., Sp. PI. 148. 1753; Clarke in Hook. f., Fl. Brit. India 3: 506. 1882; Duthie, Fl. Upp. Gang. Plain 1: 461. repr. ed. 1960; Maliya \& Datt, J. Econ. Taxon. Bot. 34 (1): 56.2010.

A. arvensis L. var. coerulea (Schreb.) Gren. \& Godr., Fl. France 2: 467. 1852; Panigrahi et al., Bull. Bot. Surv. India 11 (1 \& 2): 104. 1969.

Habit: Herb.

Specimens examined: 26.v.1954, Motipur forest, Hira Lal 13419 (LWG); 08.xii.1965, Motipur range, Katerniaghat Wildlife Sanctuary, Bahraich O.P. Mishra 7722 (BSA); 11.iii.1964, on the way to Sujauli, G. Panigrahi 2789 (BSA); 21.iii.2003, Katerniaghat range, coll. S.D. Maliya 225216 (LWG).

Embelia tsjeriam-cottam (Roem. \& Schult.) A. DC., Trans. Linn. Soc. London 17(1): 131. 1834.

Ardisia tsjeriam-cottam Roem. \& Schult., Syst. veg. 4:518. 1819

Habit: Shrub.

Specimens examined: 14.ii.1965, Uraital, Bichhiya, O.P. Mishra 7994 (BSA)

Primula umbellata (Lour.) Bentv., Fl. Malesiana, Ser 1, Spermatoph. 6: 19. 1963; Maliya \& Datt, J. Econ. Taxon. Bot. 34 (1): 56. 2010.

Drosera umbellata Lour., Fl. Cochinch. (ed. 1): 186. 1790. Androsace saxifragefolia Bunge., Mem. Ac. St. Petersb. 2: 127. 1835; Clarke in Fl. Brit. India 3: 496. 1882; Duthie, Fl. Upp. Gang. Plain 1: 460. repr. ed. 1960; Panigrahi et al., Bull. Bot. Surv. India 11 (1 \& 2): 105. 1969.
A. umbellata (Lour.) Merr., Philip. Journ. Sci. 15: 237. 1919.

Habit: Annual herb

Specimens examined: 11.iii.1964, Sujauli, G. Panigrahi 2796 (BSA); 08.ii.1965, Kharia nala, Motipur range, O.P. Mishra 7747 (BSA); 14.ii.1965, Urai nala, Bichhiya, O.P. Mishra 7986 (BSA); 16.iii.2004, Karmohini forest, coll. S.D. Maliya 225298 (LWG); 22.ii.2005, Girwa river bank, Kuthiyaghat forest, coll. S.D. Maliya 224683 (LWG); 01.iii.2011, Kailashpuri forest, coll. S.D. Maliya 214916 (LWG).

\section{Putranjivaceae}

Putranjiva roxburghii Wall., Tent. Fl. Napal. 2: 61. 1826; Hook. f. in Hook. f., Fl. Brit. India 5: 336. 1887; Duthie, Fl. Upp. Gang. Plain 3: 184. repr. ed. 1960.

Drypetes roxburghii (Wall.) Hurusawa, J. Fac. Sci. Univ. Tokyo, Sect. 3, Bot. 6: 337. 1956; Panigrahi et al., Bull. Bot. Surv. India 11 (1 \& 2): 99. 1969; Saini, J. Econ. Taxon. Bot. 29 (4): 870. 2005; Maliya \& Datt, J. Econ. Taxon. Bot. 34 (1): 62.2010

Habit: Tree.

Specimens examined: 12.iii.1964, Murtiha gate, Murtiha range, G. Panigrahi 2878 (BSA); 20.xi.1964, Nishangara range, G. Panigrahi \& O.P. Mishra 6506 (BSA); 11.ii.1965, Bichhiya, Nishangara range, O.P. Mishra 7936 (BSA).

\section{RANUNCULACEAE}

Clematis gouriana Roxb. ex DC., Prod. 1: 138. 1817; Hook. f. \& Thoms. in Hook. f., Fl. Brit. India 1: 4. 1872; Panigrahi et al., Bull. Bot. Surv. India 11 (1 \& 2): 102. 1969; Rau in Sharma et al., Fl. India 1: 64. 1993; Saini, J. Econ. Taxon. Bot. 29 (3): 533. 2005.

C. acuminata sensu Maliya \& Datt, J. Econ. Taxon. Bot. 34 (1): 46. 2010.

Habit: Climber.

Specimens examined: 24.xii.2004, Kuthiyaghat forest, coll. S.D. Maliya 225260 (LWG) (Image 54).

Naravelia zeylanica (L.) DC., Syst. 1: 167. 1818; Hook. f. \& Thoms.in Hook. f., Fl. Brit. India1: 7. 1872; Rau in Sharma et al., Fl. India 1: 106. 1993; Panigrahi et al., Bull. Bot. Surv. India 11 (1 \& 2): 102. 1969; Saini, J. Econ. Taxon. Bot. 29 (3): 533. 2005.

Atragene zeylanica L., Sp. PI. 542. 1753. Clematis napaulensis sensu Maliya \& Datt, J. Econ. Taxon. Bot. 34 (1): 46. 2010.

Habit: Woody climber.

Specimens examined: 23.xii.2004, Girijapuri forest, coll. S.D. Maliya 225239 (LWG). 
**Ranunculus cantoniensis DC., Prodr. 1: 43. 1824; Maliya \& Datt, J. Econ. Taxon. Bot. 34 (1): 46. 2010; Rau in Sharma et al., FI. India 1: 117. 1993; Srivastava, Taiwania 55 (3): 277, f. 6.2010.

R. pensylvanicus sensu Hook. f. \& Thoms. in Hook. f., Fl. Brit. India 1: 19. 1872.

Habit: Annual herb.

Specimens examined: 26.v.1954, Motipur range, Hira Lal 13442 (LWG); 22.iv.2003, Nishangara range, coll. S.D. Maliya 224979 (LWG).

**Ranunculus sceleratus L., Sp. PI. 551. 1753; Hook. f. \& Thoms in Hook. f., Fl. Brit. India1: 19. 1872; Rau in Sharma et al., FI. India 1: 128. 1993; Saini, J. Econ. Taxon. Bot. 29 (3): 533. 2005; Maliya \& Datt, J. Econ. Taxon. Bot. 34 (1): 46. 2010; Srivastava, Taiwania 55 (3): 290, f. 33 2010.

Habit: Herb.

Specimens examined: 23.iii.2004, Kuthiyaghat forest, coll. S.D. Maliya 225939 (LWG).

\section{RHAMNACEAE}

Ventilago denticulata Willd., Ges. Naturf. Fr. Neue Schr. 3: 417. 1801; Maliya \& Datt, J. Econ. Taxon. Bot. 34 (1): 49.2010.

V. maderaspatana Gaertn., Fruct. 1: 223, t. 49, f. 2. 1788; Lawson in Hook. f., Fl. Brit. India 1: 631. 1875; Saini, J. Econ. Taxon. Bot. 29 (3): 553. 2005.

Habit: Climbing shrub.

Specimens examined: 09.xii.1986, K.K. Khanna \& R. Saran 37684 (BSA); 20.iv.2003, Murtiha range, coll. S.D. Maliya 224947 (LWG).

Ziziphus mauritiana Lam., Encycl. 3(1): 319. 1789; Panigrahi et al., Bull. Bot. Surv. India 11 (1 \& 2): 100. 1969; Maliya \& Datt, J. Econ. Taxon. Bot. 34 (1): 49. 2010.

Rhamnus jujuba L., Sp. PI. 194. 1753.

Habit: Small tree or shrub.

Specimens examined: 21.xi.1964, Nishangara range, G. Panigrahi \& O.P. Mishra 6508 (BSA); 17.v.1987, K.K. Singh \& party 5945 (LWG); 20.v.1990, Jaliha, Motipur range, K.K. Khanna 64 (BSA); 25.viii.2002, Murtiha range, coll. S.D. Maliya 223737 (LWG); 09.xi.2002, road side, Katerniaghat range, coll. S.D. Maliya 224545 (LWG); 10.xi.2002, Murtiha range, coll. S.D. Maliya 224583 (LWG).

Ziziphus nummularia (Burm. f.) Wight. \& Arn., Prodr. FI. India Orient. 162. 1834; Lawson in Hook. f., Fl. Brit. India 1: 633. 1875; Maliya \& Datt, J. Econ. Taxon. Bot. 34 (1): 49. 2010.

Rhamnus nummularia Burm. f., FI. Ind. 61. 1768.
Habit: Shrub.

Specimens examined: 24.xi.1987, Rampurwa, Nishangara range, Katerniaghat Wildlife Sanctuary, Bahraich K.K. Singh \& party 6185 (LWG).

Ziziphus oenopolia (L.) Mill., Gard. Dict. (ed. 8) no. 3. 1768; Lawson in Hook. f., Fl. Brit. India 1: 634. 1875; Maliya \& Datt, J. Econ. Taxon. Bot. 34 (1): 49. 2010.

Rhamnus oenopolia L., Sp. PI. 194. 1753.

Habit: Scandent shrub.

Specimens examined: 07.xii.1986, Motipur range, K.K. Khanna \& R. Saran 38578 (BSA); 25.viii.2002, Murtiha range, coll. S.D. Maliya 223723 (LWG).

Ziziphus xylopyrus (Retz.) Willd., Sp. PI. 1104. 1798; Lawson in Hook. f., Fl. Brit. India 1: 634. 1975; Panigrahi et al., Bull. Bot. Surv. India 11 (1 \& 2): 100. 1969; Saini, J. Econ. Taxon. Bot. 29 (3): 554. 2005; Maliya \& Datt, J. Econ. Taxon. Bot. 34 (1): 49. 2010.

Rhamnus xylopyrus Retz., Observ. bot. 2: 11. 1781.

Habit: Small tree.

Specimens examined: 24.xi.1964, Nishangara range, G. Panigrahi \& O.P. Mishra 6604 (BSA); 23.viii.2002, Nishangara range, Katerniaghat Wildlife Sanctuary, coll. S.D. Maliya 223612 (LWG); 23.viii.2002, Nishangara range, coll. S.D. Maliya 223613 (LWG); 10.xi.2002, Murtiha range, Katerniaghat Wildlife Sanctuary, coll. S.D. Maliya 224852 (LWG).

\section{ROSACEAE}

Duchesnea indica (Andr.) Focke in Engler \& Prantel, Nat. Pflanzenfam. 3 (3): 33. 1888; Hara \& Ohashi, Fl. East. Himalaya, 120. 1966; Maliya \& Datt, J. Econ. Taxon. Bot. 34 (1): 52. 2010.

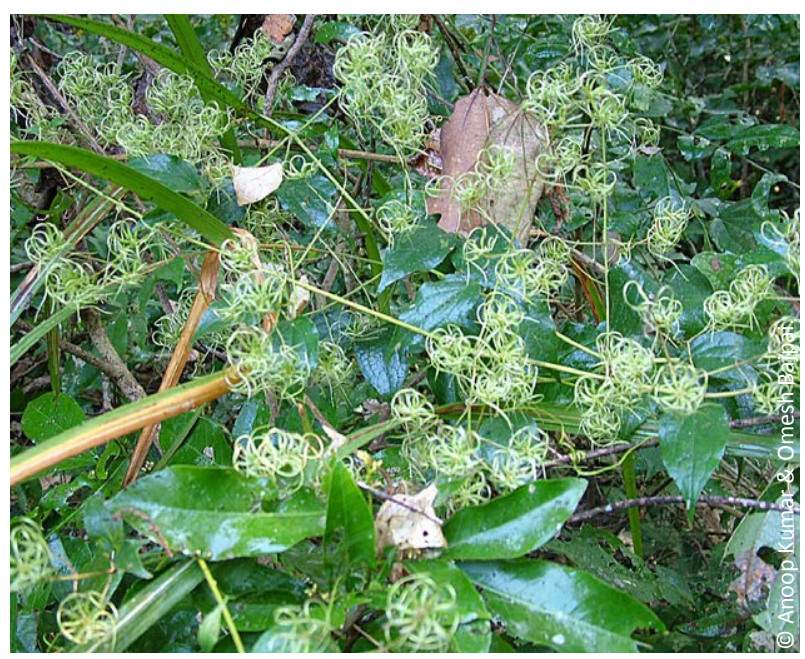

Image 54. Clematis gouriana Roxb. 
Fragaria indica Andr., Bot. Repos. 7: t. 479. 1807; Hook. f. in Hook. f., Fl. Brit. India 2: 343. 1878; Panigrahi et al., Bull. Bot. Surv. India 11 (1 \& 2): 93. 1969. Potentilla indica (Andr.) Th. Wolf in Asch. \& Graebn. Syn. Mitt. - Eur. Fl. 6 (1): 661.1904.

Habit: Herb.

Specimens examined: 12.iii.1964, Murtiha range, G. Panigrahi 2885 (BSA); 16.iii.2004, Karmohini forest, coll. S.D. Maliya 225292 (LWG); 16.iii.2003, Karmohini forest, coll. S.D. Maliya 225293 (LWG); 02.02.2011, Kailashpuri forest, coll. S.D. Maliya 214918 (LWG).

Potentilla supina L., Sp. PI. 497. 1753; Hook. f. in Hook. f., Fl. Brit. India 2: 359. 1879; Duthie, Fl. Upp. Gang. Plain 1: 301. repr. ed. 1960; Panigrahi et al., Bull. Bot. Surv. India 11 (1 \& 2): 93. 1969; Saini, J. Econ. Taxon. Bot. 29 (3): 575. 2005.

Habit: Herb.

Specimens examined: 14.ii.1965, Uraital, O.P. Mishra 7998 (BSA).

\section{RUBIACEAE}

Catunaregam spinosa (Thunb.) Trivengadum, Bull. Mus. Hist. Nat. Paris, Ser. 3, Bot. 35: 13. 1978 \& Taxon 27: 515. 1978; Verdcourt, Kew Bull. Thunb. Diss. Gard. No. 7. 1780; Saini, J. Econ. Taxon. Bot. 29 (3): 590. 2005; Maliya \& Datt, J. Econ. Taxon. Bot. 34 (1): 54.2010.

Gardenia spinosa Thunb., Gardenia. 7: 16. 1780. G. dumetorum Retz., Observ. Bot. 2: 14.1781. Randia dumetorum (Retz.) Lam., Encycl. 2: 227. 1819. Xeromphis spinosa (Thunb.) Keay, Bull. Jard. Bot. Etat Brux. 28: 37. 1958; Maheshwari, Bull. Bot. Surv. India 3: 91. 1962; Panigrahi et al., Bull. Bot. Surv. India 11 (1 \& 2): 102.1969.

Habit: Small tree.

Specimens examined: 12.ii.1965, Nishangara range, O.P. Mishra 7955 (BSA); 09.xii.1986, O.P. Mishra 37662 (BSA); 28.viii.2002, Murtiha range, coll. S.D. Maliya 223795 (LWG); 02.ix.2003, Kuthiyaghat forest, coll. S.D. Maliya 225207 (LWG); 21.xii.2003, Katerniaghat range, coll. S.D. Maliya 225217 (LWG); 16.vii.2004, Kuthiyaghat forest, coll. S.D. Maliya 225947 (LWG); 08.x.2009, road side, Murtiha range, coll. S.D. Maliya 226662 (LWG).

Ceriscoides turgida (Roxb.) Tirveng., Bull. Mus. Nat. Hist. Nat., Ser. 3, Bot. 35: 15. 1978.

Gardenia turgida Roxb., Hort. Beng. 13. 1814 \& FI. Ind. 2: 557. 1824; Hook. f. in Hook. f., Fl. Brit. India 3: 118. 1880; Maliya \& Datt, J. Econ. Taxon. Bot. 34 (1): 54. 2010; Gardenia latifolia sensu Maliya \& Datt, J. Econ. Taxon. Bot. 34 (1): 54. 2010.
Habit: Small tree.

Specimens examined: 26.viii.2002, road side, Katerniaghat range, coll. S.D. Maliya 223784 (LWG); 17.iv.2003, coll. S.D. Maliya 224903 (LWG).

Dentella repens (L.) J. R. Forst. \& G. Forst., Char. Gen. PI. 26, t. 13. 1776; Hook. f. in Hook. f., Fl. Brit. India 3: 42. 1880; Duthie, Fl. Upp. Gang. Plain 1: 378. repr. ed. 1960; Saini, J. Econ. Taxon. Bot. 29 (3): 590. 2005.

Oldenlandia repens L., Mant. PI. 1: 40. 1767.

Habit: Herb.

Specimens examined: Not observed. Record based on Saini (2005).

Haldina cordifolia (Roxb.) Ridsdale, Blumea 24 (2): 361. 1978; Maliya \& Datt, J. Econ. Taxon. Bot. 34 (1): 54. 2010.

Adina cordifolia (Roxb.) Hook. f. ex Brandis, For. Fl. N. W. \& C. India 263. 1874; Hook. f. in Hook. f., Fl. Brit. India 3: 24. 1880; Panigrahi et al., Bull. Bot. Surv. India 11 (1 \& 2): 101. 1969. Nauclea cordifolia Roxb., PI. Coromandel 1: 40, t. 53. 1795.

Habit: Tree.

Specimens examined: 12.iii.1964, Murtiha gate, G. Panigrahi 2854 (BSA); 13.iv.1967, S. L. Kapoor \& party 70034 (LWG); 26.viii.2002, coll. S.D. Maliya 223759 (LWG).

Hymenodictyon orixense (Roxb.) Mabberley, Taxon 31: 66. 1982; Maliya \& Datt, J. Econ. Taxon. Bot. 34 (1): 54. 2010.

Cinchona orixensis Roxb., Bot. Descr. Swietenia 21. 1793. C. excelsa Roxb., PI. Coromandel 2: 3, t. 106. 1799. Hymenodictyon excelsum (Roxb.) Wall. in Roxb., FI. Ind. 2: 149. 1824; Hook. f. in Hook. f., Fl. Brit. India 3: 35. 1880.

Habit: Tree.

Specimens examined: 16.iv.1967, S. L. Kapoor 70052 (LWG); 11.xi.2002, Murtiha range, coll. S.D. Maliya 224619 (LWG); 09.x.2009, road side, Kakraha range, coll. S.D. Maliya 226667 (LWG).

Knoxia sumatrensis (Retz.) DC., Prodr. 4: 569. 1830; Raizada, Ind. For. 92 (6): 315; Panigrahi et al., Bull. Bot. Surv. India 11 (1 \& 2): 101. 1969; Maliya \& Datt, J. Econ. Taxon. Bot. 34 (1): 54. 2010.

Spermacoce sumatrensis Retz., Observ. Bot. 4: 23. 1786. Knoxia corymbosa auct. pl., non Willd. 1797; Saini, J. Econ. Taxon. Bot. 29: (3): 592. 2005; Hook. f. in Hook. f., Fl. Brit. India 3: 128. 1880.

Habit: Herb.

Specimens examined: 20.xi.1964, Nishangara range, G. Panigrahi 6442 (BSA). 
Kohautia gracilis (Wall.) DC., Prodr. 4: 430. 1830.

Hedyotis gracilis Wall. in Roxb., Fl. Ind. 1: 371. 1820; Saini, J. Econ. Taxon. Bot. 29(3): 591. 2005. Oldenlandia gracilis (Wall.) Hook. f. in Fl. Brit. India 3: 68. 1880.

Habit: Herb.

Specimens examined: Not observed. Record based on Saini (2005).

Meyna laxiflora Robyns, Bull. Jard. Bot. Etat. Brux. 11: 228. 1921; Raizada, Suppl. Fl. Upp. Gang. Plain 95. 1976; Saini, J. Econ. Taxon. Bot. 29(3): 593. 2005.

Vangueria spinosa Hook. f., Fl. Brit. India 3: 136. 1880. Habit: Shrub.

Specimens examined: Not observed. Record based on Saini (2005)

Mitragyna parvifolia (Roxb.) Korth., Observ. Naucl. Indic. 19. 1839; Duthie, Fl. Upp. Gang. Plain 1: 376. repr. ed. 1960; Panigrahi et al., Bull. Bot. Surv. India 11 (1 \& 2): 101. 1969; Saini, J. Econ. Taxon. Bot. 29 (3): 593. 2005; Maliya \& Datt, J. Econ. Taxon. Bot. 34 (1): 54. 2010.

Nauclea parvifolia Roxb., PI. Coromandel 1: 40, t. 52. 1795.

Habit: Tree.

Specimens examined: 15.iii.1964, Rampurwa, Nishangara range, G. Panigrahi 2912 (BSA); 16.iv.1967, S. L. Kapoor 70053 (LWG); 16.x.1981, Motipur range, D. C. Saini 11294 (BSIP); 24.xii.2003, Kuthiyaghat forest, coll. S.D. Maliya 225251 (LWG).

**Morinda citrifolia L., Sp. PI.: 176 (1753), nom. cons.

M. tinctoria Noronha, Verh. Batav. Genootsch. Kunsten 5(4): 20. 1790. M. tomentosa Heyne ex Roth, Nov. PI. Sp. 147. 1821; Maliya, J. Econ. Taxon. Bot. 36 (2): 421. 2012.

Habit: Tree.

Specimens examined: 22.x.2010, Nishangara range, coll. S.D. Maliya 252626 (LWG).

* Mussaenda glabrata (Hook. f.) Hutch. ex Gamble, FI. Madras 610. 1921; Saini, J. Econ. Taxon. Bot. 29 (3): 593. 2005.

M. frondosa var. glabrata Hook. f., in Fl. Brit. India 3: 90. 1880 .

Habit: Rambling shrub, cultivated.

Specimens examined: Not observed. Record based on Saini (2005).

Neanotis calycina (Wall. ex Hook. f.) Lewis, Ann. Miss. Bot. Gard. 53: 37. 1966; Maliya \& Datt, J. Econ. Taxon. Bot. 34 (1): 54.2010.
Anotis calycina Hook. f. in Hook. f., Fl. Brit. India 3: 73. 1880.

Habit: Herb.

Specimens examined: Not observed. Record based on Maliya \& Datt (2010).

Neolamarckia cadamba (Roxb.) Bosser, Bull. Mus. Natl. Hist. Nat., B, Adansonia 6: 247. 1984; Maliya \& Datt, J. Econ. Taxon. Bot. 34 (1): 54. 2010.

Nauclea cadamba Roxb., Fl. Ind. 2: 121. 1824. Anthocephalus cadamba (Roxb.) Miq., Fl. Ned. Ind. 2: 135. 1856. A. chinensis sensu Saini, J. Econ. Taxon. Bot. 29 (3): 589. 2005.

Habit: Tree.

Specimens examined: 11.xi.2002, Murtiha range, coll. S.D. Maliya 224606 (LWG); 14.iii.2010, Nepal border, Rampurwa forest, coll. S.D. Maliya 252610 (LWG).

Oldenlandia biflora L., Sp. PI. 119. 1753.

O. paniculata L., Sp. PI. 1667. 1753; Panigrahi et al., Bull. Bot. Surv. India 11 (1 \& 2): 102. 1969.

Habit: Herb.

Specimens examined: 21.xi.1964, Nishangara range, G. Panigrahi \& O.P. Mishra 6522 (BSA); 14.ii.1965, Uraital, Bichhia, G. Panigrahi 7990 (BSA).

Oldenlandia corymbosa L., Sp. PI. 119. 1753; Hook. f. in Hook. f., Fl. Brit. India 3: 64. 1880; Duthie, Fl. Upp. Gang. Plain 1: 380. repr. ed. 1960; Panigrahi et al., Bull. Bot. Surv. India 11 (1 \& 2): 101. 1969.

Hedyotis corymbosa (L.) Lam., Encycl. 1: 272. 1792; Rao \& Hemadri, Indian For. 99: 375. 1973; Maliya \& Datt, J. Econ. Taxon. Bot. 34 (1): 54. 2010.

Habit: Herb.

Specimens examined: 16.ii.1965, Katerniaghat range, G. Panigrahi 8018 (BSA); 09.xi.2002, rest house, coll. S.D. Maliya 224528 (LWG).

Oldenlandia verticillata L., Mant. PI. 1: 40. 1767.

Hedyotis hispida Retz., Observ. Bot. 4: 23. 1786; Hook. f. in Hook. f., Fl. Brit. India 3: 60. 1880. H. verticillata (L.) Lam., Encycl. 1: 271. 1791; Saini, J. Econ. Taxon. Bot. 29: (3): 592.2005.

Habit: Herb.

Specimens examined: Not observed. Record based on Saini (2005).

Spermacoce articularis L. f., Suppl. PI. 119. 1781.

Borreria articularis (L. f.) F. N. Williams in Bull. Herb. Boiss. ser. 2, 5: 956. 1905; Panigrahi et al., Bull. Bot. Surv. India 11 (1 \& 2): 101.1969. 
Habit: Procambent herb.

Specimens examined: 22.xi.1964, Orla, Motipur range, G. Panigrahi \& O.P. Mishra 6584 (BSA).

Spermacoce pusila Wall. in Roxb. Fl. Ind. 1: 379. 1820. Borreria pusilla (Wall.) DC., Prodr. 4: 543. 1830; Saini, J. Econ. Taxon. Bot. 29 (3): 590. 2005.

Habit: Herb.

Specimens examined: Not observed. Record based on Saini (2005)

Tamilnadia uliginosa (Retz.) Tirveng. \& Sastre. Mauritius Inst. Bull. 8 (4): 85. 1979.

Gardenia uliginosa Retz., Observ. Bot. 2: 14. 1781. Randia uliginosa (Retz.) Poir., Encycl. suppl. 2: 829. 1812 "Grandia uliginosa". Xeromphis uliginosa (Retz.) Maheshw., Bull. Bot. Soc. Univ. Saugar 10: 39. (1958) publ. 1961; Bot. Surv. India 3:92. 1961.

Habit: Small tree.

Specimens examined: 21.v.1987, Katerniaght wildlife sanctuary, Bahraich, K.K. Singh \& Party 5989 (LWG).

Wendlandia heynei (Roem. \& Schult.) Santapau \& Merchant, Bull. Bot. Surv. India 3: 110. 1962; Maliya \& Datt, J. Econ. Taxon. Bot. 34 (1): 55. 2010.

Rondeletia heynei Roem. \& Schult., Syst. Veg. 5: 234. 1819. R. exserta Roxb., FI. Ind. 2: 135. 1824. Wendlandia exserta (Roxb.) DC., Prodr. 4: 411. 1830; Hook. f. in Hook. f., in FI. Brit. India 3: 37. 1880.

Habit: Tree.

Specimens examined: 11.iv.2006, near Bicchiya barrier, coll. S.D. Maliya 226608 (LWG); 24.iv.2008, near Bichhiya barrier, Nishangara range, coll. S.D. Maliya 218581 (LWG).

\section{RUTACEAE}

Aegle marmelos (L.) Correa, Trans. Linn. Soc. 5: 223. 1800; Hook. f. in Hook. f., Fl. Brit. India 1: 516. 1875; Duthie, Fl. Upp. Gang. Plain 1: 135. repr. ed. 1960; Panigrahi et al., Bull. Bot. Surv. India 11 (1 \& 2): 100. 1969; Nair \& Nayar in Hajra et al., FI. India 4: 264. 1997; Maliya \& Datt, J. Econ. Taxon. Bot. 34 (1): 48. 2010.

Crataeva marmelos L., Sp. PI. 444. 1753.

Habit: Tree.

Specimens examined: 28.xii.2005, Kuthiyaghat forest, coll. S.D. Maliya 226601 (LWG).

*Citrus aurantiifolia (Christm.) Swingle, Jour. Wash. Acad. 3: 465. 1913; Nair \& Nayar in Hajra et al., Fl. India 4: 278. 1997; Maliya \& Datt, J. Econ. Taxon. Bot. 34 (1): 49. 2010.
Limonia aurantifolia Christm. \& Panz., Pflanzenfam. 1: 618. 1777. Citrus acida Roxb., Fl. Ind. 3: 390. 1832. C. medica L. var. acida Hook. f. in Hook. f., Fl. Brit. India 1: 515. 1875.

Habit: Small tree, cultivated.

Specimens examined: 18.iii.2004, Katerniaghat range, coll. S.D. Maliya 225912 (LWG).

*Citrus medica L., Sp. PI. 782. 1753; Nair \& Nayar in Hajra et al., Fl. India 4: 284. 1997; Panigrahi et al., Bull. Bot. Surv. India 11 (1 \& 2): 100. 1969.

Habit: Small tree, cultivated.

Specimens examined: 12.iii.1964, Murtiha gate, Murtiha range, G. Panigrahi 2874 (BSA); 24.xi.1987, Nishangara range, Katerniaghat wildlife sancyuary, Bahraich, K.K. Singh \& party 6178 (LWG).

**Clausena pentaphylla (Roxb.) DC., Prodr. 1: 538. 1824; Hook. f. in Hook. f., Fl. Brit. India 1: 503. 1875; Maliya \& Datt, J. Econ. Taxon. Bot. 34 (1): 49. 2010; Saini, J. Econ. Taxon. Bot. 29 (3): 551. 2005.

Amyris pentaphylla Roxb., Fl. Ind. 2: 247. 1832. Clausena kanpurensis J. F. Molino, Bull. Mus. Hist. Nat. (Paris) 16: 117, f. 2, 8-9. 1994; Nair \& Nayar in Hajra et al., FI. India 4: 284. 1997.

Habit: Shrub.

Specimens examined: 23.viii.2002, Nishangara range, coll. S.D. Maliya 223647 (LWG); 11.xi.2002, Murtiha range, coll. S.D. Maliya 224604 (LWG); 19.iv.2003, Murtiha range, coll. S.D. Maliya 224942 (LWG).

Glycosmis pentaphylla (Retz.) DC., Prodr. 1: 538. 1824; Nair \& Nayar in Hajra et al., Fl. India 4: 343. 1997; Maliya \& Datt, J. Econ. Taxon. Bot. 34 (1): 49. 2010.

Limonia pentaphylla Retz., Observ. bot. 5: 24. 1789. L. arborea Roxb., PI. Coromandel 1: 60, t. 85. 1795. Glycosmis mauritiana sensu Maliya \& Datt, J. Econ. Taxon. Bot. 34 (1): 49. 2010.

Habit: Shrub.

Specimens examined: 26.v.1954, Motipur range, Hira Lal 13455 (LWG); 10.xi.2002, Murtiha range, coll. S.D. Maliya 224592 (LWG); 12.xi.2002, Nishangara range, coll. S.D. Maliya \& K.K. Singh 224627 (LWG); 13.xi.2002, Nishangara range, K.K. Singh \& coll. S.D. Maliya 224669 (LWG); 14.xi.2002, rest house, Murtiha range, coll. S.D. Maliya 224670 (LWG); 18.iv.2003, Bichhiya town, coll. S.D. Maliya 224934 (LWG); 19.iv.2003, Murtiha range, coll. S.D. Maliya 224937 (LWG); 22.iv.2003, Nishangara range, coll. S.D. Maliya 224981 (LWG); 22.xii.2004, Kuthiyaghat forest, coll. S.D. Maliya 225997 (LWG); 04.iii.2011, rest house, Kakraha range, coll. S.D. Maliya 214923 (LWG) 
(Image 55).

Murraya koenigii (L.) Spreng., Syst. 2: 315. 1826; Hook. f. in Hook. f., Fl. Brit. India 1: 503. 1875; Duthie, FI. Upp. Gang. Plain 1: 129. repr. ed. 1960; Panigrahi et al., Bull. Bot. Surv. India 11 (1 \& 2): 100. 1969; Nair \& Nayar in Hajra et al., FI. India 4: 351. 1997; Maliya \& Datt, J. Econ. Taxon. Bot. 34 (1): 49. 2010.

Bergera koenigii L., Mant. PI. 2: 563. 1771.

Habit: Shrub.

Specimens examined: 09.iii.1963, Sohelwa, Nishangara range, G. Panigrahi 2776 (BSA) (Image 56).

Note: In USDA Germplasm Resources Information Network (GRIN) Bergera koenigii L. is still accepted name.

**Murraya paniculata (L.) Jack., Mal. Misc. 1 (5): 31. 1820; Nair \& Nayar in Hajra et al., Fl. India 4: 352. 1997; Saini, J. Econ. Taxon. Bota. 29 (3): 551. 2005.

Chalacas paniculata L., Mant. Pl. 1: 68. 1767. Murraya exotica L., Mant. PI. 2: 563. 1771; Hook. f. in Hook. f., FI. Brit. India 1: 502. 1875.

Habit: Shrub.

Specimens examined: 26.v.1954, Motipur range, Hira Lal 13464 (LWG); 24.viii.2002, Nishangara range, coll. S.D. Maliya 223684 (LWG); 17.iv.2003, road side, Katerniaghat range, coll. S.D. Maliya 224908 (LWG);

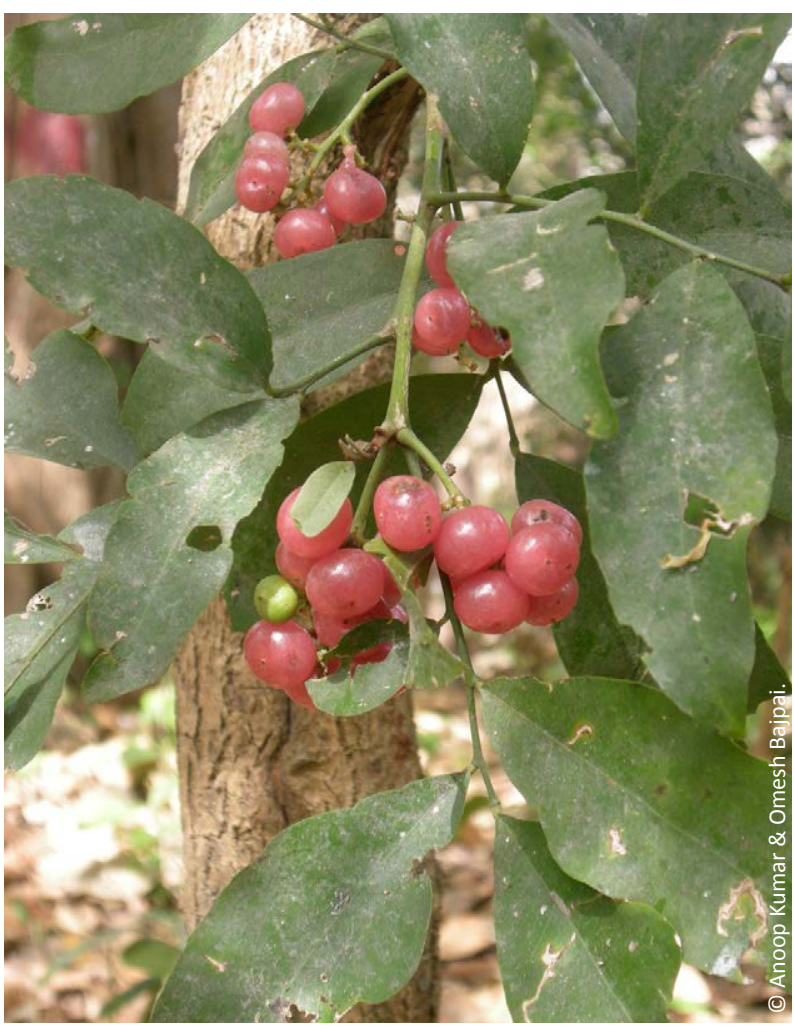

Image 55. Glycosmis pentaphylla (Retz.) DC. 19.iv.2003, Murtiha range, coll. S.D. Maliya 224939 (LWG); 22.iv.2003, Nishangara range, coll. S.D. Maliya 224975 (LWG); 16.vii.2004, Kuthiyaghat forest, coll. S.D. Maliya 225956 (LWG); 07.ix.2005, Kailashpuri forest, coll. S.D. Maliya 227159 (LWG).

\section{SALICACEAE}

**Flacourtia indica (Burm. f.) Merr., Interpr. Herb. Amboin. 377. 1917; Panigrahi et al., Bull. Bot. Surv. India 11 (1 \& 2): 97. 1969; Mitra in Sharma et al., Fl. India 2: 402. 1993.

Gmelina indica Burm. f., Fl. Ind. 132, t. 39, f. 5. 1768.

Specimens examined: 12.iii.1964, Murtiha range, G. Panigrahi 2879 (BSA).

Habit: Tree.

**Guidonia tomentosa (Roxb.) Kurz, J. Asiat. Soc. Bengal Pt. 2, Nat. Hist. 46 (2): 92.1877.

Casearia tomentosa Roxb., Fl. Ind. 2: 421. 1832; Clarke in Hook. f., Fl. Brit. India 2: 593. 1879; Mitra in Sharma et al., FI. India 2: 397. 1993. C. elliptica Willd., Sp. PI. 2: 628. 1799 (nom. superfl.); Panigrahi et al., Bull. Bot. Surv. India 11 (1 \& 2): 97. 1969; Saini, J. Econ. Taxon. Bot. 29 (3): 582. 2005; Maliya \& Datt, J. Econ. Taxon. Bot. 34 (1): 47. 2010.

Habit: Tree.

Specimens examined: 11.iii.1964, Motipur range, G. Panigrahi 2821 (BSA); 14.iv.1967, S. L. Kapoor \& party 70039 (LWG); 28.viii.2003, Murtiha range, coll. S.D. Maliya 214853 (LWG).

Salix denticulata Anders., Kongl. Svenska Vetenskapsakad. Handl., n.s. 1850: 481. 1851; Maliya \& Datt, J. Econ. Taxon. Bot. 34 (1): 64. 2010.

Salix elegans Wall. ex Andersson, J. Linn. Soc., Bot. 4: 51. 1860.

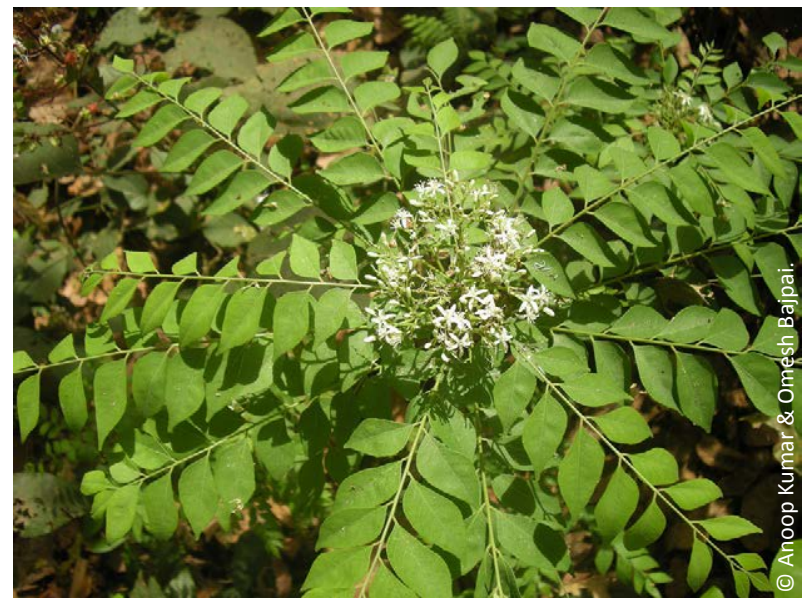

Image 56. Murraya koenigii (L.) 
Habit: Tree.

Specimens examined: 24.xii.2003, Kuthiyaghat forest, coll. S.D. Maliya 225246 (LWG); 07.xi.2008, Kuthiyaghat forest, coll. S.D. Maliya 226648 (LWG).

Salix tetrasperma Roxb., PI. Coromandel 1: 66, t. 97. 1795 \& Fl. Ind. 3: 573. 1832; Hook. f. in Hook. f., Fl. Brit. India 5: 626. 1888; Duthie, Fl. Upp. Gang. Plain 3: 253. repr. ed. 1960; Panigrahi et al., Bull. Bot. Surv. India 11 (1 \& 2): 96.1969.

Habit: Tree.

Specimens examined: 22.iv.1964, On the way to Orla, Motipur, G. Panigrahi \& O.P. Mishra 6541 (BSA).

Xylosma longifolia Clos, Ann. Sci. Nat. Bot. Ser. 4, 8: 231. 1857; Hook. f. \& Thoms. in Hook. f., Fl. Brit. India 1: 194. 1872; Mitra in Sharma et al., FI. India 2: 436. 1993; Saini, J. Econ. Taxon. Bot. 29 (3): 540. 2005.

Habit: Tree.

Specimens examined: Not observed. Record based on Saini (2005).

\section{SAPINDACEAE}

Lepisanthes rubiginosa (Roxb.) Leenh., Blumea 17 (1): 82. 1969

Sapindus rubiginosus Roxb., PI. Coromandel 1: 44, t. 62. 1795. Erioglossum rubiginosum (Roxb.) Bl., Rumphia 3: 118. 1847; Panigrahi et al., Bull. Bot. Surv. India 11 (1 \& 2): 100.1969.

Habit: Tree.

Specimens examined: Not observed. Record based on Panigrahi et al. (1969).

Schleichera oleosa (Lour.) Merr., Interpr. Rumph. Herb. Amboin. 337. 1917\& J. Arn. Arb. 31: 284. 1950.

Schleichera oleosa (Lour.) Oken., Allg. Naturgesch. 3(2): 1341. 1841; Panigrahi et al., Bull. Bot. Surv. India 11 (1 \& 2): 100. 1969; Maliya \& Datt, J. Econ. Taxon. Bot. 34 (1): 50. 2010. Pistacia oleosa Lour., Fl. Cochinch. 2: 615. 1790.

Habit: Tree.

Specimens examined: 21.v.1987, K.K. Singh \& party 5947 (LWG); 26.xi.1987, Nishangara range, K.K. Singh \& party 6912 (LWG); 20.iii.2004, Girijapuri forest, coll. S.D. Maliya 225917 (LWG) (Image 57).

\section{SAPOTACEAE}

Madhuca longifolia (L.) Macbr. var. latifolia (Roxb.) Chev., Rev. Bot. Appl. Agric. Trop. 23: 149. 1943; Maliya \& Datt, J. Econ. Taxon. Bot. 34 (1): 56. 2010.

Madhuca latifolia (Roxb.) J. F. Macbr., Contr. Gray
Herb. 53:17. 1918. M. indica J. F. Gmel., Syst. Nat. 2(1): 799. 1791. Bassia latifolia Roxb., PI. Coromandel 1: 20, t. 19. 1795; Duthie, Fl. Upp. Gang. Plain 1: 465. repr. ed. 1960.

Habit: Tree.

Specimens examined: 19.iv.2003, Murtiha range, coll. S.D. Maliya 224935 (LWG); 22.iv.2003, Nishangara range, coll. S.D. Maliya 224978 (LWG).

Manilkara hexandra (Roxb.) Dub., Ann. Mus. Colon. Marseille (Ser. 3) 3: 9, f. 2. 1915; Panigrahi et al., Bull. Bot. Surv. India 11 (1 \& 2): 100. 1969.

Mimusops hexandra Roxb., Pl. Coromandel 1: 16, t. 15. 1795 \& Fl. Ind. 2: 238. 1832; Clarke in Hook. f., Fl. Brit. India 3: 549. 1882; Duthie, Fl. Upp. Gang. Plain 1: 467. repr. ed. 1960.

Habit: Tree.

Specimens examined: 12.iii.1964, Murtiha range, G. Panigrahi 2880 (BSA).

\section{SCROPHULARIACEAE}

Mella hamiltoniana (Benth.) Pennell, Scroph. W. Himal. Acad. Nat. Sci. Philadelphia Monogr. 5: 23. 1943; Raizada in Suppl. Fl. Upp. Gang. Plain 189. 1976; Saini, J. Econ. Taxon. Bot. 29 (3): 634. 2005.

Herpestis hamiltoniana Benth., Scroph. Ind. 30. 1835; Hook. f. in Hook. f., Fl. Brit. India 4: 272. 1884.

Habit: Herb.

Specimens examined: Not observed. Record based on Saini (2005).

\section{SIMAROUBACEAE}

Ailanthus excelsa Roxb., PI. Coromandel 1: t. 23. 1795; Bennett in Hook. f. Fl. Brit. India. 1: 518. 1875; Duthie, Fl. Upp. Gang. Plain 1: 136. repr. ed. 1960; Basak in Hajra et

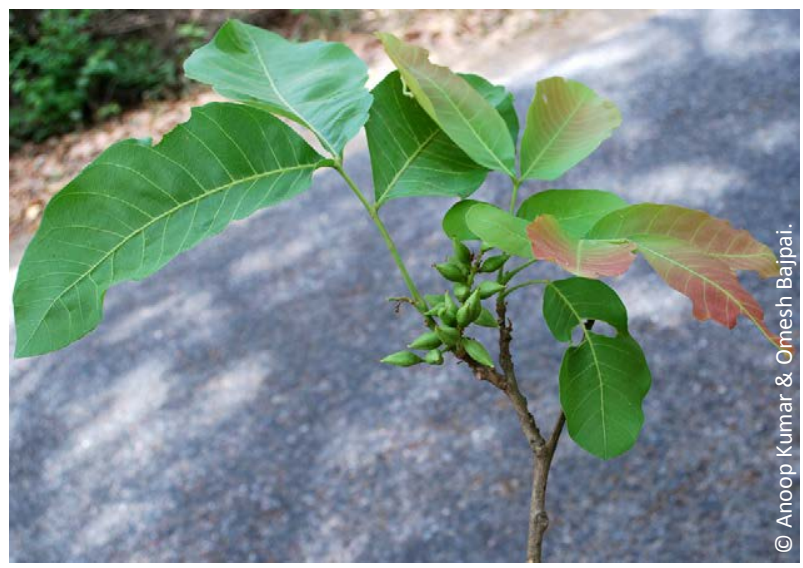

Image 57. Schleichera oleosa (Lour.) Merr. 
al., Fl. India 4: 410. 1997.

Habit: Tree.

Specimens examined: 13.xi.2011. Motipur range, A. Kumar \& O. Bajpai 252788 (LWG).

\section{SOLANACEAE}

*Capsicum annuum L., Sp. PI. 188. 1753; Panigrahi et al., Bull. Bot. Surv. India 11 (1 \& 2): 106. 1969.

Habit: Herb, cultivated.

Specimens examined: 21.xi.1964, Nishangara range, G. Panigrahi \& O.P. Mishra 6519 (BSA).

Cestrum diurnum L., Sp. PI. 191. 1753; Maliya \& Datt, J. Econ. Taxon. Bot. 34 (1): 58. 2010.

Habit: Shrub.

Specimens examined: 26.viii.2002, Katerniaghat range, coll. S.D. Maliya 223768 (LWG); 09.xi.2002, road side, Katerniaghat range, coll. S.D. Maliya 224563 (LWG); 21.xii.2003, Katerniaghat range, coll. S.D. Maliya 225219 (LWG); 24.x.2004, Girijapuri forest, coll. S.D. Maliya 227108 (LWG).

*Cestrum nocturnum L., Sp. PI. 191. 1753; Maliya \& Datt, J. Econ. Taxon. Bot. 34 (1): 58. 2010.

Habit: Shrub, cultivated.

Specimens examined: 24.iv.2003, Chhawni, Murtiha range, coll. S.D. Maliya 214836 (LWG).

**Datura innoxia Mill., Gard. Dict. ed. 8. n. 5. 1768; Raizada Suppl. Fl. Upp. Gang. Plain. 169. 1976; Metel Sims in Curtis Bot. Mag. T. 1440. 1812; Panigrahi et al., Bull. Bot. Surv. India 11 (1 \& 2): 106. 1969.

Habit: Shrub.

Specimens examined: 24.xi.1987, Nishangara range, K.K. Singh \& party 6177 (LWG).

Datura metel L., Sp. PI. 179. 1753; Duthie, FI. Upp. Gang. Plain 2: 9. repr. ed. 1960; Panigrahi et al., Bull. Bot. Surv. India 11 (1 \& 2): 106. 1969; Maliya \& Datt, J. Econ. Taxon. Bot. 34 (1): 58. 2010.

Habit: Herb.

Datura innoxia sensu Panigrahi et al., Bull. Bot. Surv. India 11 (1 \& 2): 106. 1969.

Specimens examined: 20.xi.1964, Nishangara range, G. Panigrahi \& O.P. Mishra 6448 (BSA); 11.ii.1965, Bichhiya, Nishangara range, G. Panigrahi \& O.P. Mishra 7931 (BSA); 11.xi.2002, Murtiha range, coll. S.D. Maliya 224622 (LWG).

**Nicotiana plumbaginifolia Viv., Elench. PI. Hort. Dinegro 26, t. 5. 1802, nom. cons.; Raizada in Suppl.
Fl. Upp. Gang. Plain. 170. 1976; Maliya \& Datt, J. Econ. Taxon. Bot. 34 (1): 58. 2010.

Habit: Herb.

Specimens examined: 24.viii.2002, Nishangara range, coll. S.D. Maliya 223678 (LWG); 23.iii.2004, Kuthiyaghat forest, coll. S.D. Maliya 225940 (LWG).

Physalis minima L., Sp. PI. 183. 1753; Clarke in Hook. f., Fl. Brit. India 4: 238. 1883; Duthie, Fl. Upp. Gang. Plain 2: 5. repr. ed. 1960; Panigrahi et al., Bull. Bot. Surv. India 11 (1 \& 2): 106. 1969; Maliya \& Datt, J. Econ. Taxon. Bot. 34 (1): 58. 2010.

Habit: Herb.

Specimens examined: 11.iii.1964, Motipur range, G. Panigrahi 2832 (BSA); 08.ix.2005, Kuthiyaghat forest, coll. S.D. Maliya 227163 (LWG); 27.xii.2005, Lahaura village, Nishangara range, coll. S.D. Maliya 227198 (LWG); 11.iii.2010, Karikot marg, Nishangara range, coll. S.D. Maliya 252604 (LWG).

Solanum anguivi Lam., Encycl. 2:23. 1794; Maliya \& Datt, J. Econ. Taxon. Bot. 34 (1): 58. 2010.

Habit: Shrub.

Specimens examined: 09.xi.2002, Katerniaghat range, coll. S.D. Maliya 224546 (LWG); 16.vii.2004, Kuthiyaghat forest, coll. S.D. Maliya 225958 (LWG).

**Solanum donianum Walp., Repert. Bot. Syst. 3: 54. 1844.

S. verbascifolium L., Sp. Pl. 184. 1753; Clarke in Hook. f., Fl. Brit. India 4: 230. 1883; Duthie, FI. Upp. Gang. Plain 2: 2. repr. ed. 1960; Panigrahi et al., Bull. Bot. Surv. India 11 (1 \& 2): 106. 1969.

Habit: Shrub or Small tree.

Specimens examined: 15.iii.1964, Rampurwa, Nishangara range, G. Panigrahi 2909 (BSA); 11.ii.1965, Bichhiya, Nishangara range, O.P. Mishra 7919 (BSA).

**Solanum erianthum D. Don, Prodr. FI. Nepal. 96. 1825; Maliya \& Datt, J. Econ. Taxon. Bot. 34 (1): 58. 2010. Habit: Shrub.

Specimens examined: 09.xi.2002, coll. S.D. Maliya 224517 (LWG); 23.xii.2003, Girijapuri forest, coll. S.D. Maliya 225241 (LWG); 20.vii.2004, Girijapuri forest, coll. S.D. Maliya 225971 (LWG); 29.xii.2005, Kuthiyaghat forest, coll. S.D. Maliya 226604 (LWG).

**Solanum incanum L., Sp. PI. 188. 1753; Duthie, Fl. Upp. Gang. Plain 2: 4. repr. ed. 1960; Maliya \& Datt, J. Econ. Taxon. Bot. 34 (1): 58. 2010.

Habit: Shrub. 
Specimens examined: 29.xii.2005, Kuthiyaghat forest, coll. S.D. Maliya 226607 (LWG).

Solanum lasiocarpum Dunal, Hist. nat. Solanum 222 1813.

Solanum ferox sensu Maliya, J. Econ. Taxon. Bot. 36 (2): 422. 2012.

Habit: Shrub.

Specimens examined: 09.iii.2010, Nishangara range, Katerniaghat Wildlife Sanctuary, Bharaich, coll. S.D. Maliya 226696 (LWG).

Solanum nigrum L., Sp. PI. 186. 1753; Clarke in Hook. f., Fl. Brit. India 4: 229. 1883; Duthie, Fl. Upp. Gang. Plain 2: 2. repr. ed. 1960; Panigrahi et al., Bull. Bot. Surv. India 11 (1 \& 2): 106. 1969; Saini, J. Econ. Taxon. 29 (4): 628 2005; Maliya \& Datt, J. Econ. Taxon. Bot. 34 (1): 58. 2010. Habit: Herb.

Specimens examined: 11.iii.1964, Motipur range, G. Panigrahi 2839 (BSA); 21.xi.1964, Motipur on the way to Orla, G. Panigrahi \& O.P. Mishra 6564 (BSA); 09.xi.2002, near Bichhiya, coll. S.D. Maliya 224518 (LWG).

Solanum torvum Sw., Prodr. 47. 1788; Clarke in Hook. f. Fl. Brit. India 4: 234. 1883; Saini, J. Econ. Taxon. Bot. 29 (3): 628. 2005.

Habit: Shrub.

Specimens examined: Not observed. Record based on Saini (2005)

**Solanum viarum Dunal in DC., Prodr. 13 (1): 240. 1852; Raizada in Suppl. Fl. Upp. Gang. Plain 177. 1976; Maliya \& Datt, J. Econ. Taxon. Bot. 34 (1): 58. 2010.

Solanum khasianum Clarke var. chatterjeeanum Sengupta, Bull. Bot. Surv. India 3: 413. 1961.

Habit: Undershrub.

Specimens examined: 16.v.1987, K.K. Singh \& party 5942 (LWG); 25.xi.1987, Nishangara range, K.K. Singh \& party 6189 (LWG); 23.viii.2002, Nishangara range, coll. S.D. Maliya 223664 (LWG); 10.xi.2002, Murtiha range, coll. S.D. Maliya 224594 (LWG); 14.xi.2002, rest house, Murtiha range, coll. S.D. Maliya 224676 (LWG); 02.ix.2003, Kuthiyaghat forest, coll. S.D. Maliya 225203 (LWG); 17.iii.2004, Kuthiyaghat forest, coll. S.D. Maliya 225905 (LWG); 16.iii.2008, Murtiha range, coll. S.D. Maliya 218517 (LWG).

Solanum virginianum L., Sp. PI. 187. 1753; Maliya \& Datt, J. Econ. Taxon. Bot. 34 (1): 58. 2010.

S. surattense Burm. f., Fl. Ind. 57. 1768, pp.; Saini, J.
Econ. Taxon. Bot. 29 (3): 628. 2005; Panigrahi et al., Bull. Bot. Surv. India 11 (1 \& 2): 106. 1969. S. xanthocarpum Schrad. in Schrad. \& Wendl., Sert. Hanov. 1: 8. 1795; Clarke in Hook. f., Fl. Brit. India 4: 236. 1883; Duthie, Fl. Upp. Gang. Plain 2: 3. repr. ed. 1960.

Habit: Herb.

Specimens examined: 26.v.1954, Motipur range, Hira Lal 13422 (LWG); 26.viii.2002, coll. S.D. Maliya 223771 (LWG); 22.iv.2003, Nishangara range, coll. S.D. Maliya 224971 (LWG); 18.iii.2004, Kuthiyaghat forest, coll. S.D. Maliya 225908 (LWG); 25.iii.2008, Kuthiyaghat forest, coll. S.D. Maliya 218580 (LWG).

\section{STERCULIACEAE}

Firmiana colorata (Roxb.) R. Br., Pterocymbium 235. 1844 \& in Benn. \& Brown, Pl. jav. rar. 235. 1844; Malick in Sharma et al., Fl. India 3: 420. 1993; Saini, J. Econ. Taxon. Bot. 29 (3): 546. 2005.

Sterculia colorata Roxb., Pl. Coromandel 1: 26. 1975; Mast. in Hook. f., Fl. Brit. India 1: 359. 1874.

Habit: Tree.

Specimens examined: Not observed. Record based on Saini (2005).

Guazuma ulmifolia Lam., Encycl. 3: 52. 1789; Malick in Sharma et al., Fl. India 3: 424. 1993; Saini, J. Econ. Taxon. Bot. 29 (3): 546. 2005.

Theobroma guazuma L., Sp. PI. 782. 1753.

Habit: Tree.

Specimens examined: Not observed. Record based on Saini (2005).

Helicteres isora L., Sp. PI. 963. 1753; Mast. in Hook. f., Fl. Brit. India 1: 365. 1874; Duthie, Fl. Upp. Gang. Plain 1: 96. repr. ed. 1960; Panigrahi et al., Bull. Bot. Surv. India 11 (1 \& 2): 98. 1969; Malick in Sharma et al., Fl. India 3: 426. 1993; Saini, J. Econ. Taxon. Bot. 29 (3): 546. 2005; Maliya \& Datt, J. Econ. Taxon. Bot. 34 (1): 48. 2010.

Habit: Shrub or small tree.

Specimens examined: 11.ii.1965, Bichhia, Nishangara range, O.P. Mishra 7938 (BSA); 12.xii.1986, Motipur range, K.K. Khanna \& R. Saran 38659 (BSA); 23.viii.2002, Nishangara range, coll. S.D. Maliya 223658 (LWG); 12.xi.2002, Nishangara range, coll. S.D. Maliya 224658 (LWG); 29.viii.2003, rest house, Nishangara range, coll. S.D. Maliya 214860 (LWG).

Melochia corchorifolia L., Sp. PI. 675. 1753 nom. cons.; Mast. in Hook. f., Fl. Brit. India 1: 374. 1874; Malick in Sharma et al., Fl. India 3: 441. 1993; Saini, J. Econ. Taxon. Bot. 29 (3): 546. 2005. 
Habit: Herb.

Specimens examined: Not observed. Record based on Saini (2005).

Sterculia foetida L., Sp. PI. 1008. 1753; Mast. in Hook f., FI. Brit. India 1: 354. 1874; Malick in Sharma et al., FI. India 3: 459. 1993; Saini, J. Econ. Taxon. Bot. 29 (3): 547. 2005.

Habit: Tree.

Specimens examined: Not observed. Record based on Saini (2005)

Sterculia urens Roxb., PI. Coromandel 1: 25, t. 24. 1795; Panigrahi et al., Bull. Bot. Surv. India 11 (1 \& 2): 98. 1969; Malick in Sharma et al., Fl. India 3: 470. 1993; Maliya \& Datt, J. Econ. Taxon. Bot. 34 (1): 48. 2010.

Habit: Tree.

Specimens examined: 14.ii.1965, Uraital, Bichhiya, Nishangara range, O.P. Mishra 7995 (BSA).

Sterculia villosa Roxb. ex Sm., Rees Cycl. 34: Sterculia no. 16. 1816; Panigrahi et al., Bull. Bot. Surv. India 11 (1 \& 2): 98. 1969; Malick in Sharma et al., Fl. India 3: 472. 1993; Maliya \& Datt, J. Econ. Taxon. Bot. 34 (1): 48. 2010

Habit: Tree.

Specimens examined: 14.iv1967, S. L. Kapoor \& party 70040 (LWG); 26.xi.1987, K.K. Singh \& party 6190 (LWG); 17.iv.2003, road side, Katerniaghat range, Katerniaghat Wildlife Sanctuary, coll. S.D. Maliya 224902 (LWG); 02.ix.2003, Girijapuri forest, coll. S.D. Maliya 214895 (LWG); 24.x.2004, Katerniaghat range, coll. S.D. Maliya 227109 (LWG); 24.ii.2009, rampurwa rest house, Nishangara range, L.B. Chaudhary, A. Kumar \& O. Bajpai 250271 (LWG); 15.iv.2009, grassland, Katerniaghat range, L.B. Chaudhary, A. Kumar \& O. Bajpai 252181 (LWG); 06.vi.2009, rampurwa rest house, Nishangara range, L.B. Chaudhary, A. Kumar \& O. Bajpai 250614 (LWG); 09.iii.2011, Katerniaghat range, A. Kumar \& O. Bajpai 252898 (LWG).

\section{TAMARIACACEAE}

**Tamarix dioica Roxb. ex Roth, Nov. PI. Sp. 185. 1821; Duthie, Fl. Upper Gangetic Plain 1: 68. 1903; Panigrahi et al., Bull. Bot. Surv. India 11 (1 \& 2): 97. 1969.

Habit: Shrub.

Specimens examined: Not observed. Record based on Panigrahi et al. (1969).

Tamarix indica Willd., Ges. Naturf. Freunde Berlin Neue Schriften. 4: 214. 1803; Shetty \& Pandey in Sharma et al., FI. India 3: 25. 1993.

T. troupii Hole, Indian Forest. 45: 248. 1919; Panigrahi et al., Bull. Bot. Surv. India 11 (1 \& 2): 97. 1969.

Habit: Tree.

Specimens examined: Not observed. Record based on Panigrah et al. (1969).

\section{Ulmaceae}

Holoptelea integrifolia (Roxb.) Planch., Ann. Sci. Nat. (Ser. 3) 10: 259. 1848; Hook. f. in Hook. f., Fl. Brit. India 5: 481. 1888; Duthie, Fl. Upp. Gang. Plain 3: 217. repr. ed. 1960; Panigrahi et al., Bull. Bot. Surv. India 11 (1 \& 2): 96. 1969; Maliya \& Datt, J. Econ. Taxon. Bot. 34 (1): 63.2010. Ulmus integrifolia Roxb., Pl. Coromandel 1: 56, t. 78. 1975.

Habit: Tree.

Specimens examined: 11.iii.1964, Motipur range, G. Panigrahi 2830 (BSA); 18.iv.2003, road side, Katerniaghat range, coll. S.D. Maliya 224931 (LWG).

\section{URTICACEAE}

Gonostegia pentandra (Roxb.) Miq., Ann. Mus. Bot. Lugduno-Batavi 4: 302. 1868. 1869.

Urtica pentandra Roxb., FI. Ind. 3: 583. 1832. Pouzolzia pentandra (Roxb.) Benn., Pl. Jav. Rar. 64, 66, t. 14. 1838; Duthie, Fl. Upp. Gang. Plain 3: 227. repr. ed. 1960; Panigrahi et al., Bull. Bot. Surv. India 11 (1 \& 2): 97. 1969; Maliya \& Datt, J. Econ. Taxon. Bot. 34 (1): 63. 2010.

Habit: Herb.

Specimens examined: Not observed. Record based on Panigrahi et al. (1969) and Maliya \& Datt (2010).

Pouzolzia zeylanica (L.) Benn. \& R. Br., PI. Jav. Rar. 66 - 67. 1838; Maliya \& Datt, J. Econ. Taxon. Bot. 34 (1): 63. 2010.

Parietaria zeylanica L., Sp. PI. 1052. 1753. P. indica L., Mant. PI. 1: 128. 1767. Pouzolzia indica (L.) Gaud., Voy. Bonite, Bot. 503. 1826; Hook. f. in Hook. f., Fl. Brit. India 5: 581. 1888; Duthie, Fl. Upp. Gang. Plain 3: 226. repr. ed. 1960.

Habit: Herb.

Specimens examined: Not observed. Record based on Maliya \& Datt (2010).

Urtica dioica L., Sp. PI. 984. 1753; Maliya \& Datt, J. Econ. Taxon. Bot. 34 (1): 63. 2010.

Habit: Herb or undershrub.

Specimens examined: 24.iv.2003, bank of Girwa river, Katerniaghat range, coll. S.D. Maliya 214840 (LWG); 21.xii.2003, bank of Girwa river, Katerniaghat range, coll. S.D. Maliya 225218 (LWG). 


\section{VIOLACEAE}

Viola cinerea Boiss., Diagn. PI. Orient. Ser. 1, 1: 7. 1843; Panigrahi et al., Bull. Bot. Surv. India 11 (1 \& 2): 97. 1969.

Habit: Perennial herb.

Specimens examined: Not observed. Record based on Panigrahi et al. (1969).

\section{VITACEAE}

Ampelocissus latifolia (Roxb.) Planch, Vigne-Amer. Vitic. Eur. 8: 374. 1884; Saini, J. Econ. Taxon. Bot. 29 (3): 554. 2005; Maliya \& Datt, J. Econ. Taxon. Bot. 34 (1): 49. 2010.

Vitis latifolia Roxb., FI. Ind. 2: 474. 1824.

Habit: Climber.

Specimens examined: 25.viii.2002, Murtiha range, K.K. Singh \& coll. S.D. Maliya 223714 (LWG).

Cayratia trifolia (L.) Domin, Biblioth. Bot. 89: 370. 1927.

Vitis trifolia L., Sp. Pl. 203. 1753; Duthie, Fl. Upp. Gang. Plain 1: 163. repr. ed. 1960. Cissus carnosa Lam., Encycl. 1: 31. 1783. Cayratia carnosa (Lam.) Gagnep., Notul. Syst. (Paris) 1: 347. 1911; Panigrahi et al., Bull. Bot. Surv. India 11 (1 \& 2): 100. 1969.

Habit: Climber.

Specimens examined: 21.xi.1964, Nishangara range, G. Panigrahi \& O.P. Mishra 6525 (BSA); 09.xii.1986, K.K. Khanna \& R. Saran 37688 (BSA).

Leea alata Edgew., Trans. Linn. Soc. London 20 (1): 36. 1846; Maliya \& Datt, J. Econ. Taxon. Bot. 34 (1): 50. 2010. Habit: Shrub.

Specimens examined: 24.viii.2002, Nishangara range, coll. S.D. Maliya 223670 (LWG).

Leea asiatica (L.) Ridsdale, Bot. Hist. Hort. Malab. 189. 1980; Maliya \& Datt, J. Econ. Taxon. Bot. 34 (1): 50. 2010.

Phytolacca asiatica L., Sp. PI. 441. 1753. Leea crispa L., Syst. Nat. ed. 12, 2: 627. 1767; Lawson in Hook. f., Fl. Brit. India 1: 665. 1875. L. herbacea Ham., Trans. Linn. Soc. 14 228. 1823. L. aspera Edg., Trans. Linn. Soc. 20: 36. 1846. L. pumila Kurz, J. Asiat. Soc. Beng. 4 (2): 302. 1872; Lawson in Hook. f., Fl. Brit. India 1: 666. 1875. L. edgeworthi Santapau, Rec. Bot. Surv. India 16: 54. 1953; Panigrahi et al., Bull. Bot. Surv. India 11 (1 \& 2): 100. 1969.

Habit: Shrub.

Specimens examined: 22.xi.1964, Nishangara range, G. Panigrahi \& O.P. Mishra 6485 (BSA); 26.xi.1987, K.K. Singh \& party 6194 (LWG); 23.viii.2002, Nishangara range, coll. S.D. Maliya 223642 (LWG); 29.viii.2003, Nishangara range, coll. S.D. Maliya 214863 (LWG); 02.ix.2003, Kuthiyaghat forest, coll. S.D. Maliya 214900 (LWG); 24.xii.2003, Kuthiyaghat forest, coll. S.D. Maliya 225262 (LWG).

Leea indica (Burm. f.) Merr., Philip. J. Sci. 14 (2): 245. 1919; Saini, J. Econ. Taxon. Bot. 29 (3): 555. 2005.

Staphylea indica Burm. f., Fl. Ind. 75, t. 24. f. 2. 1768.

Habit: Shrub.

Specimens examined: Not observed. Record based on Saini (2005).

Tetrastigma leucostaphylum (Dennst.) Alston ex Mabb.,Taxon 26: 539. 1977.

Cissus leucostaphyla Dennst., Schlussel Hort. Malab. 17, 19, 33. 1818. Tetrastigma lanceolarium (Roxb.) Planch. in DC., Monog. Phan. 5: 423. 1887; Saini, J. Econ. Taxon. Bot. 29 (3): 555. 2005. Cissus lanceolraria Roxb., Fl. Ind. 1: 412. 1820.

Habit: Climber.

Specimens examined: Not observed. Record based on Saini (2005).

\section{MONOCOTYLEDONS}

\section{ALISMATACEAE}

**Alisma plantago-aquatica L., Sp. PI. 342. 1753; Hook. f. in Hook. f., Fl. Brit. India 6: 559. 1893; Saini, J. Econ. Taxon. Bot. 29 (4): 890. 2005.

Habit: Herb.

Specimens examined: Not observed. Record based on Saini (2005).

Caldesia oligococca (Muell.) Buchenau, Bot. Jahrb. Syst. 2: 479. 1882; Panigrahi et al., Bull. Bot. Surv. India 11 (1 \& 2): 109. 1969.

Alisma oligococcum Muell., Fragm. 1: 23. 1858; Duthie, FI. Upp. Gang. Plain 3: 370. repr. ed. 1960.

Habit: Herb.

Specimens examined: 22.xi.1964, Motipur on the way to Orla, G. Panigrahi \& O.P. Mishra 6549 (BSA).

Caldesia parnassifolia (Bassi ex L.) Parl., Fl. Ital. 3: 599. 1860.

Alisma parnassifolia Bassi ex L., Syst. Nat. 3: 230. 1768. Alisma reniforme D. Don, Prodr. Fl. Nepal. 22. 1825. Habit: Herb.

Specimens examined: 27.v.1954, Motipur range, Hira Lal 13473 (LWG) (Image 58). 
**Sagittaria guayanensis Kunth in F.W.H.A. von Humboldt et al., Nov. Gen. Sp. 1: 250. 1816; Hook. f. in Hook. f., Fl. Brit. India 6: 501. 1893; Duthie, Fl. Upp. Gang. Plain 3: 372. repr. ed. 1960; Saini, J. Econ. Taxon. Bot. 29 (4): 891. 2005.

Habit: Herb.

Specimens examined: Not observed. Record based on Saini (2005).

**Sagittaria sagittifolia L., Sp. PI. 993. 1753; Hook. f. in Hook. f., Fl. Brit. India 6: 561. 1893; Duthie, Fl. Upp. Gang. Plain 3: 371. repr. ed. 1960; Panigrahi et al., Bull. Bot. Surv. India 11 (1 \& 2): 109. 1969; Saini, J. Econ. Taxon. Bot. 29 (4): 891. 2005.

Habit: Herb.

Specimens examined: 25.v.1954, Motipur range, Hira Lal 13474 (LWG); 12.iii.1964, Motipur range, G. Panigrahi 2846 (BSA); 27.ii.2011, Harrayya village, Katerniaghat wildlife sanctary, Bahraich, coll. S.D. Maliya 214905 (LWG).

**Sagittaria sagittifolia L. subsp. leucopetala (Miq.) Hartog, Steenis, Fl. males. ser. 1, 5(3): 332. 1957.

S. sagittifolia L. var. leucopetala Miq., III. fl. Archip. Ind. 2: 49. 1870. S. trifolia L., Sp. PI. 2:293. 1753; Maliya, J. Econ. Taxon. Bot. 36 (1): 161. 2012.

Note: Sometimes (according to Plantlist, Tropicos and Kew-list) Sagittaria trifolia L. is treated as a distinct species.

Habit: Herb.

Specimens examined: 22.iv.2003, Nishangara range, Katerniaghat wildlife sanctary, Bahraich, coll. S.D. Maliya 224982 (LWG).

\section{AMARYLLIDACEAE}

*Crinum asiaticum L., Sp. PI. 292. 1753; Duthie, FI. Upp. Gang. Plain 3: 320. repr. ed. 1960; Maliya \& Datt, J. Econ. Taxon. Bot. 34 (1): 64. 2010.

Habit: Herb, cultivated.

Specimens examined: 12.xi.2002, Nishangara range, coll. S.D. Maliya 224661 (LWG); 28.xii.2003, Near Bichhiya railway station, coll. S.D. Maliya 225278 (LWG).

\section{APONOGETONACEAE}

Aponogeton crispus Thunb., Nov. Gen. PI. 73: 1784; Panigrahi et al., Bull. Bot. Surv. India 11 (1 \& 2): 109.1969. Habit: Aquatic herb.

Specimens examined: 24.xi.1964, Nishangara range, G. Panigrahi \& O.P. Mishra 6604 (BSA).

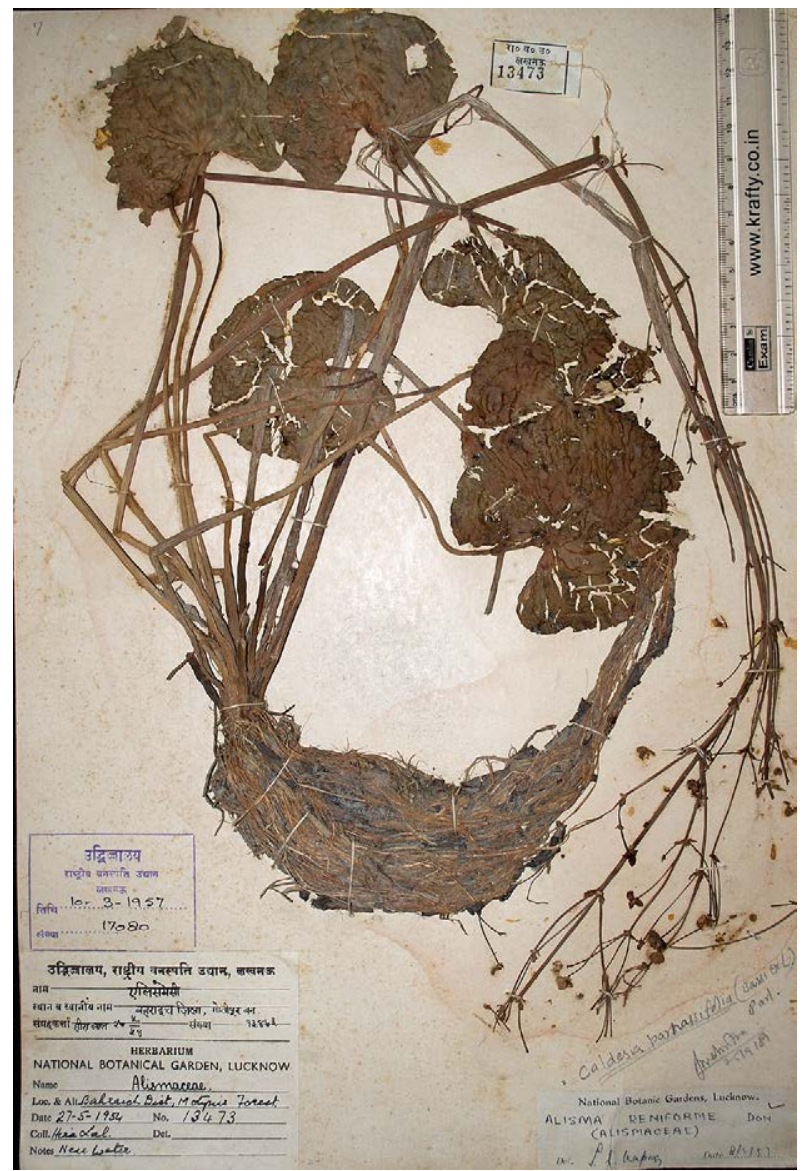

Image 58. Caldesia parnassifolia (Bassi ex L.) Parl.

\section{ARACEAE}

**Alocasia macrorrhizos (L.) G. Don in R. Sweet, Hort. Brit. 3: 631. 1839; Maliya \& Datt, J. Econ. Taxon. Bot. 34 (1): 66. 2010.

Arum macrorrhizon L., Sp. Pl. 965. 1753. A. indicum Lour., Fl. Cochinch. 2: 536. 1790. Alocasia indica (Lour.) Spach, Hist. Nat. Veg. 12: 47. 1846.

Habit: Aquatic herb.

Specimens examined: 27.viii.2003, Girwa river bank, Katerniaghat range, Katerniaghat wildlife sanctary, Bahraich, coll. S.D. Maliya 214843 (LWG); 16.vii.2004, Kuthiyaghat forest, Katerniaghat wildlife sanctary, Bahraich, coll. S.D. Maliya 225952 (LWG).

Amorphophallus paeoniifolius (Dennst.) Nicolson, Taxon 26: 338. 1977; Maliya \& Datt, J. Econ. Taxon. Bot. 34 (1): 66. 2010.

Dracontium paeoniaefolium Dennst., Schlussel Hortus Malab. 38. 1818

Habit: Herb.

Specimens examined: 27.viii.2003, Katerniaghat range, Katerniaghat wildlife sanctary, Bahraich, coll. S.D. 
Maliya 214842 (LWG); 23.xii.2005, Katerniaghat range, Katerniaghat wildlife sanctary, Bahraich, coll. S.D. Maliya 227182 (LWG).

**Pistia stratiotes L., Sp. PI. 963. 1753; Hook. f. in Hook. f., Fl. Brit. India 6: 497. 1893; Duthie, FI. Upp. Gang. Plain 3: 361. repr. ed. 1960; Panigrahi et al., Bull. Bot. Surv. India 11 (1 \& 2): 110. 1969; Saini, J. Econ. Taxon. Bot. 29 (4): 889. 2005.

Habit: Aquatic herb.

Specimens examined: 22.xi.1964, Motipur on the way to Orla, G. Panigrahi \& O.P. Mishra 6548 (BSA).

\section{AreCACEAe (Palmae nom. alt.)}

Calamus tenuis Roxb., Fl. Ind. 3: 780. 1832; Beccari \& Hook. f. in Hook. f., Fl. Brit. India 6: 447. 1892; Duthie, Fl. Upp. Gang. Plain 3: 356. repr. ed. 1960; Panigrahi et al., Bull. Bot. Surv. India 11 (1 \& 2): 110. 1969; Maliya \& Datt, J. Econ. Taxon. Bot. 34 (1): 65. 2010.

C. royleanus Griff., Calcutta J. Nat. Hist. 5: 40. 1845.

Habit: Scandent shrub.

Specimens examined: 18.iv.2003, Bichhiya checkpost, Katerniaghat wildlife sanctary, Bahraich, coll. S.D. Maliya 224959 (LWG); 21.iv.2003, Nishangara range, Katerniaghat wildlife sanctary, Bahraich, coll. S.D. Maliya 224960 (LWG); 02.ix.2003, Kuthiyaghat forest, Katerniaghat wildlife sanctary, Bahraich, coll. S.D. Maliya 225204 (LWG); 26.xii.2005, near Bichhiya railway line, Katerniaghat wildlife sanctary, Bahraich, coll. S.D. Maliya 227184 (LWG) (Image 59).

Phoenix acaulis Roxb., (Hort. Bengal. 73. 1814 nom. nud.) PI. Coromandel 3: 69, t. 273. 1819; Duthie, Fl. Upp. Gang. Plain 3: 354. repr. ed. 1960; Maliya \& Datt, J. Econ. Taxon. Bot. 34 (1): 65. 2010.

Habit: Shrub.

Specimens examined: 22.iii.2004, Nishangara range, Katerniaghat wildlife sanctary, Bahraich, coll. S.D. Maliya 225934 (LWG).

Phoenix loureiroi Kunth., Enum. PI. 3: 257. 1841.

P. humilis Royle ex Becc., Malesia 3: 373. 1890; Duthie, FI. Upp. Gang. Plain 3: 355. repr. ed. 1960; Maliya \& Datt, J. Econ. Taxon. Bot. 34 (1): 65. 2010.

Habit: Shrub.

Specimens examined: 24.viii.2002, Nishangara range, Katerniaghat wildlife sanctary, Bahraich, coll. S.D. Maliya 223683 (LWG).

\section{ASPARAGACEAE}

*Agave americana L., Sp. pl. 1: 323. 1753; Maliya, J. Econ. Taxon. Bot. 36 (2): 424. 2012.

Habit: Herb, cultivated.

Specimens examined: 16.iii.2004, Karmohini forest, Katerniaghat range, coll. S.D. Maliya 225289 (LWG).

*Agave cantala (Haw.) Roxb. ex Salm-Dyck, Index PI. Succ. Hort. Dyck. 1. 1829; Maliya, J. Econ. Taxon. Bot. 36 (2): 424. 2012.

Furcraea cantala Haw., Suppl. PI. Succ. 42. 1818.

Habit: Herb, cultivated.

Specimens examined: 19.vii.2004, Kuthiyaghat forest, coll. S.D. Maliya 225976 (LWG).

*Asparagus adscendens Roxb., Fl. ind. ed. 1832, 2:153. 1832

Habit: Suberect shrub, cultivated.

Specimens examined: 28.viii.2011, rest house, Nishangara range, coll. S.D. Maliya 214910 (LWG).

Asparagus racemosus Willd., Sp. PI. 2: 152. 1799; Hook. f. in Hook. f., Fl. Brit. India 6: 316. 1892; Duthie, Fl. Upp. Gang. Plain 3: 327. repr. ed. 1960; Maliya \& Datt, J. Econ. Taxon. Bot. 34 (1): 65. 2010.

Habit: Scandent shrub.

Specimens examined: 24.viii.2002, Nishangara range, coll. S.D. Maliya 223669 (LWG).

Chlorophytum tuberosum (Roxb.) Baker, J. Linn. Soc. Bot. 15: 332. 1877; Maliya \& Datt, J. Econ. Taxon. Bot. 34 (1): 65. 2010.

Anthericum tuberosum Roxb., PI. Coromandel 2: 20. 1800.

Habit: Herb.

Specimens examined: Not observed. Record based on

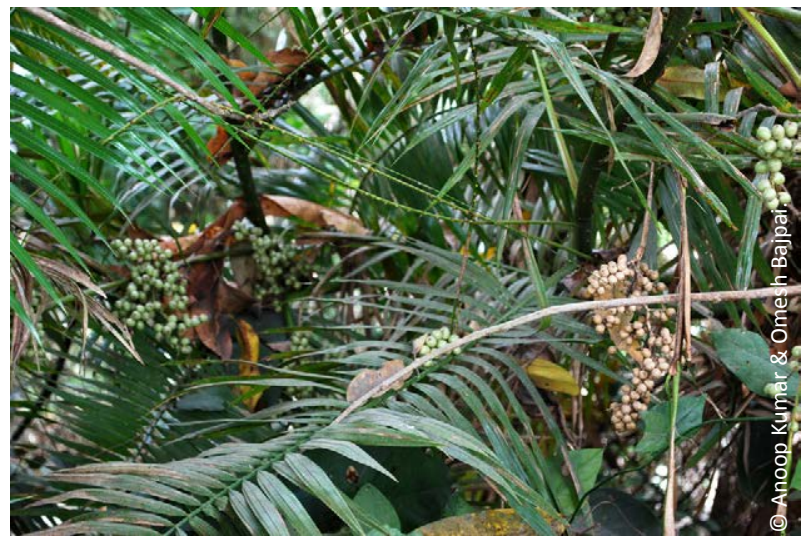

Image 59. Calamus tenuis Roxb. 
Maliya \& Datt (2010).

Drimia indica (Roxb.) Jessop, J. S. African Bot. 43 (4): 272. 1977; Maliya \& Datt, J. Econ. Taxon. Bot. 34 (1): 65 2010.

Scilla indica Roxb., FI. Ind. 2: 147. 1832; Duthie, Fl. Upp. Gang. Plain 3: 332. repr. ed. 1960.

Habit: Herb.

Specimens examined: 25.xii.2003, Nishangara range, coll. S.D. Maliya 225271 (LWG).

\section{CANNACEAE}

*Canna indica L., Sp. PI. 1: 1. 1753; Maliya, J. Econ. Taxon. Bot. 36 (2): 423.2012.

Habit: Herb, cultivated.

Specimens examined: 09.x.2009, rest house, Kakraha range, coll. S.D. Maliya 226668 (LWG).

\section{COLCHICACEAE}

Gloriosa superba L., Sp. PI. 305. 1753; Duthie, Fl. Upp. Gang. Plain 3: 330. repr. ed. 1960; Panigrahi et al., Bull. Bot. Surv. India 11 (1 \& 2): 110. 1969.

Habit: Climbing herb.

Specimens examined: 24.xi.1964, Katerniaghat range, G. Panigrahi \& O.P. Mishra 6613A (BSA) (Image 60).

\section{COMmelinACEAE}

Commelina caroliniana Walter, Fl. Carol. 68. 1788.

C. hasskarlii Clarke, Commelyn. Cyrtandr. Bengal. 13, t. 3. 1874; Duthie, Fl. Upp. Gang. Plain 3: 339. repr. ed. 1960; Panigrahi et al., Bull. Bot. Surv. India 11 (1 \& 2): 109. 1969; Maliya \& Datt, J. Econ. Taxon. Bot. 34 (1): 65. 2010.

Habit: Herb.

Specimens examined: 21.iv.1964, Nishangara, G. Panigrahi \& O.P. Mishra 6530 (BSA); 26.x.2004, Nepal border, Murtiha range, coll. S.D. Maliya 227113 (LWG).

Commelina maculata Edgew., Trans. Linn. Soc. London 20 (1): 89. 1851; Maliya \& Datt, J. Econ. Taxon. Bot. 34 (1): 65. 2010.

C. obliqua var. viscida Clarke in DC., Monogr. Phan. 3: 178. 1881. C. paludosa var. viscida (Clarke) Rao \& Kamathy, Bull. Bot. Surv. India 3: 168. 1961.

Habit: Herb.

Specimens examined: 23.viii.2002, Nishangara range, coll. S.D. Maliya 223629 (LWG); 23.viii.2002, Nishangara range, coll. S.D. Maliya 223660 (LWG); 26.x.2004, Nepal border, Murtiha range, coll. S.D. Maliya 227122 (LWG).

Cyanotis axillaris (L.) D. Don. ex Sweet, Hort. Brit. 430. 1826.

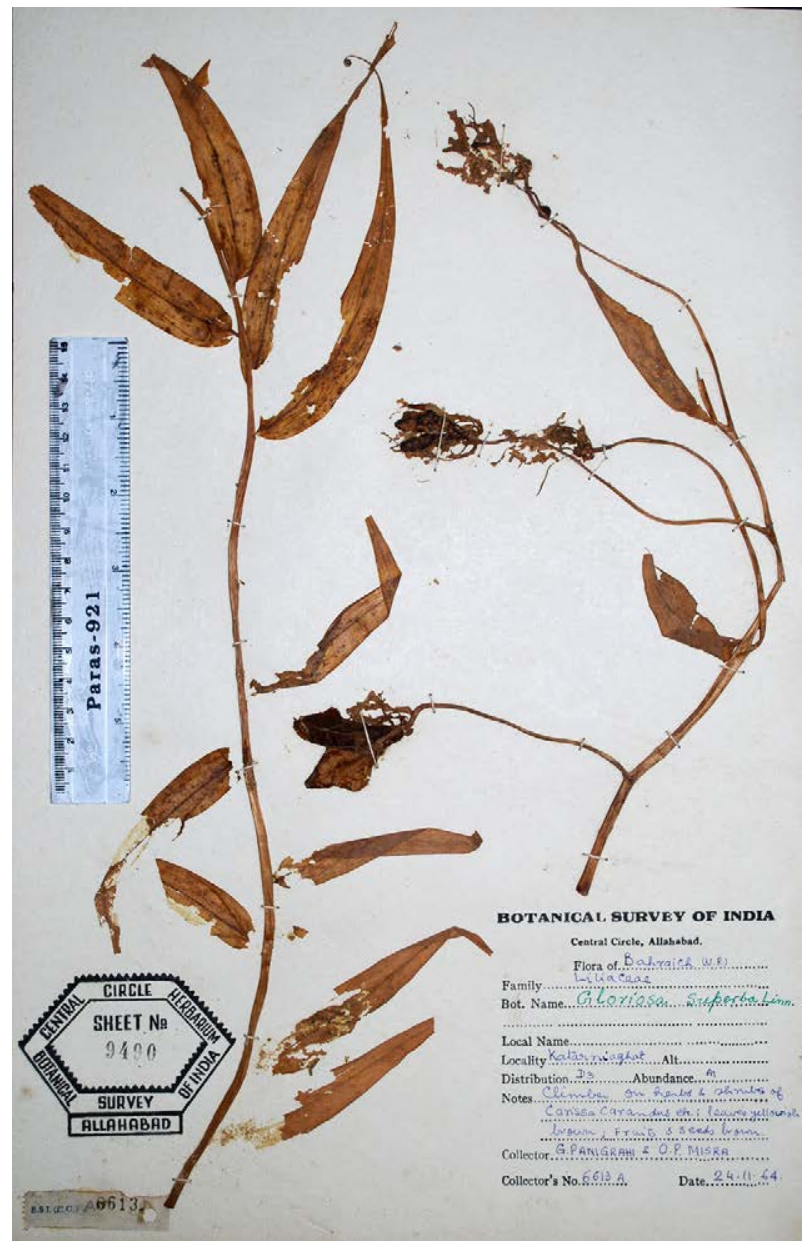

Image 60. Gloriosa superba $\mathrm{L}$.

Commelina axillaris L., Sp. PI. 42. 1753. Tradescantia axillaris (L.) L., Mant. PI. 2: 321. 1771. Amischophacelus axillaris (L.) Rao \& Kammathy, J. Linn. Soc. Bot. 59 (379): 306. 1966; Saini, J. Econ. Taxon. Bot. 29 (4): 886. 2005; Maliya \& Datt, J. Econ. Taxon. Bot. 34 (1): 65. 2010.

Habit: Herb.

Specimens examined: 26.x.2004, Nepal border, Murtiha range, coll. S.D. Maliya 227114 (LWG).

Cyanotis cristata (L.) D. Don, Prodr. Fl. Nepal. 46. 1825; Duthie, FI. Upp. Gang. Plain 3: 343. repr. ed. 1960; Maliya \& Datt, J. Econ. Taxon. Bot. 34 (1): 65. 2010.

Commelina cristata L., Sp. PI. 42. 1753.

Habit: Herb.

Specimens examined: 11.xi.2002, Murtiha range, coll. S.D. Maliya 224602 (LWG).

**Murdannia nudiflora (L.) Brenan, Kew Bull. 7 (2): 189. 1952; R. Rao, Bull. Bot. Surv. Ind. 3 (3 \& 4): 393. 1961; Maliya \& Datt, J. Econ. Taxon. Bot. 34 (1): 65. 2010. 
Commelina nudiflora L., Sp. PI. 41. 1753; Duthie, Fl. Upp. Gang. Plain 3: 338. repr. ed. 1960. Aneilema nudiflorum R. Br., Prodr. 271. 1810; Hook. f. in Hook. f., FI. Brit. India 6: 378. 1892; Duthie, FI. Upp. Gang. Plain 3: 342. repr. ed. 1960.

Habit: Herb.

Specimens examined: Not observed. Record based on Maliya \& Datt (2010).

Murdannia spirata (L.) Brueckner, Nat. Pfl.- Syst. 15a: 173. 1930; Panigrahi et al., Bull. Bot. Surv. India 11 (1 \& 2): 109.1969.

Commelina spirata L., Mant. PI. 2: 176. 1771.

Habit: Herb.

Specimens examined: 24.xi.1964, Nishangara range, G. Panigrahi \& O.P. Mishra 6597 (BSA).

\section{CYPERACEAE}

**Bolboschoenus maritimus subsp. affinis (Roth) Koyama, Brittonia 31 (2): 284. 1979.

Scirpus affinis Roth in Roem. \& Schult., Syst. Veg. 2: 140. 1817. S. maritimus L. var. affinis Clarke in Hook. f., Fl. Brit. India 6: 659. 1893; Duthie, Fl. Upp. Gang. Plain 3: 412. repr. ed. 1960; Panigrahi et al., Bull. Bot. Surv. India 11 (1 \& 2): 111. 1969.

Habit: Herb.

Specimens examined: 09.ii.1965, Jairampurwa, O.P. Mishra 7761 (BSA).

Bulbostylis barbata (Rottb.) Clarke in Hook. f., Fl. Brit. India 6 (19): 651. 1893; Duthie, Fl. Upp. Gang. Plain 3: 408. repr. ed. 1960; Maliya \& Datt, J. Econ. Taxon. Bot. 34 (1): 66. 2010

Scirpus barbatus Rottb., Descr. Icon. Rar. PI. 27. 1772. Habit: Herb.

Specimens examined: 29.viii.2003, rest house, Nishangara range, coll. S.D. Maliya 214859 (LWG).

Carex fedia Nees in Wight, Contr. Bot. India 129. 1834

C. wallichiana Prescott. ex Nees in Wight., (Wall., Cat. n. 3380. 1831) Contrib. Bot. India 129. 1834 (non Spreng); Clarke in Hook. f., Fl. Brit. India 6: 747. 1894; Duthie, FI. Upp. Gang. Plain 3: 418. repr. ed. 1960; Panigrahi et al., Bull. Bot. Surv. India 11 (1 \& 2): 110. 1969.

Habit: Herb.

Specimens examined: Not observed. Record based on Panigrahi et al. (1969).

**Carex heterostachya Bunge, Enum. PI. China Bor. 69. 1833; Maliya \& Datt, J. Econ. Taxon. Bot. 34 (1): 66. 2010.
Habit: Herb.

Specimens examined: 22.iv.2003, Nishangara range, coll. S.D. Maliya 224990 (LWG); 02.iii.2011, Kailashpuri forest, Nishangara range, coll. S.D. Maliya 214917 (LWG).

Cyperus castaneus Willd., Sp. PI. 1: 278. 1797; Saini, J. Econ. Taxon. Bot. 29 (4): 894. 2005.

Habit: Herb.

Specimens examined: Not observed. Record based on Saini (2005).

Cyperus compactus Retz., Observ. Bot. 5: 10. 1788.

C. dilutus Vahl, Enum. PI. 2: 357. 1805; Panigrahi et al., Bull. Bot. Surv. India 11 (1 \& 2): 110. 1969.

Habit: Herb.

Specimens examined: 23.xi.1964, Kharia nala, Motipur range, O.P. Mishra 6574 (BSA).

**Cyperus compressus L., Sp. PI. 46. 1753; Clarke in Hook. f., Fl. Brit. India 6: 605. 1893; Duthie, Fl. Upp. Gang. Plain 3: 384. repr. ed. 1960.; Maliya \& Datt, J. Econ. Taxon. Bot. 34 (1): 66. 2010.

Habit: Herb.

Specimens examined: 25.viii.2002, Murtiha range, coll. S.D. Maliya 223697 (LWG).

**Cyperus cuspidatus Kunth in F. W. H. A. von Humboldt et al., Nov. Gen. Sp. 1: 204. 1816; Hook. f. in Hook. f., Fl. Brit. India 6: 598. 1893; Saini, J. Econ . Taxon. Bot. 29 (6): 894. 2005.

C. uncinatus Poir., Encycl. 7: 247. 1806; Duthie, Fl. Upp. Gang. Plain 3: 381. repr. ed. 1960.

Habit: Herb.

Specimens examined: Not observed. Record based on Saini (2005).

Cyperus cyperoides (L.) Kuntze, Revis. Gen. PI. 3(3): 333. 1898; Saini, J. Econ. Taxon. Bot. 29 (4): 894. 2005.

Scirpus cyperoides L., Mant. PI. 2: 181. 1771. Kyllinga sumatrensis Retz., Observ. Bot. 4: 13. 1786. Mariscus sumatrensis (Retz.) Raynal, Adansonia, n.s., 15: 110. 1975; Maliya \& Datt, J. Econ. Taxon. Bot. 34 (1): 66. 2010.

Habit: Herb.

Specimens examined: 23.viii.2002, Nishangara range, coll. S.D. Maliya 223615 (LWG); 24.viii.2002, Nishangara range, coll. S.D. Maliya 223692 (LWG); 25.viii.2002, Murtiha range, coll. S.D. Maliya 223706 (LWG).

**Cyperus difformis L., Cent. PI. 2: 6. 1756; Clarke in Hook. f., Fl. Brit. India 6: 599. 1893; Duthie, Fl. Upp. Gang. 
Plain 3: 382. repr. ed. 1960; Panigrahi et al., Bull. Bot. Surv. India 11 (1 \& 2): 110. 1969.

Habit: Herb.

Specimens examined: 09.ii.1965, Rampurwa, O.P. Mishra 7797 (BSA).

Cyperus digitatus Roxb., Fl. Ind. 1: 209. 1820; Clarke in Hook. f., Fl. Brit. India 6: 618. 1893; Duthie, Fl. Upp. Gang. Plain 3: 387. repr. ed. 1960; Saini, J. Econ. Bot. 29 (6): 895. 2005.

Habit: Herb.

Specimens examined: Not observed. Record based on Saini (2005).

Cyperus distans L. f., Suppl. PI. 103. 1782; Clarke in Hook. f., Fl. Brit. India 6: 607. 1893; Duthie, Fl. Upp. Gang. Plain 3: 390. repr. ed. 1960; Saini, J. Econ. Bot. 29 (4): 895. 2005; Maliya \& Datt, J. Econ. Taxon. Bot. 34 (1): 66. 2010. Habit: Herb.

Specimens examined: 23.viii.2002, Nishangara range, coll. S.D. Maliya 223616 (LWG); 25.viii.2003, Murtiha range, coll. S.D. Maliya 223702 (LWG).

Cyperus dubius Rottb., Descr. Icon. Rar. PI. 20. 1773.

Kyllinga triceps Rottb., Descr. Icon. Rar. PI. 14, t. 4, f. 6. 1773; Clarke in Hook. f., Fl. Brit. India 6: 587. 1893; Duthie, Fl. Upp. Gang. Plain 3: 396. repr. ed. 1960. Cyperus triceps (Rottb.) Endl., Cat. Horti. Vindob. 1: 94. 1842; Saini, J. Econ. Taxon. Bot. 29 (4): 898. 2005.

Habit: Herb.

Specimens examined: Not observed. Record based on Saini (2005).

Cyperus exaltatus Retz., Observ. Bot. 5: 11.1788 ; Clarke in Hook. f., Fl. Brit. India 6: 617. 1893; Duthie, FI. Upp. Gang. Plain 3: 387. repr. ed. 1960; Saini, J. Econ. Taxon. Bot. 29 (6): 895. 2005; Maliya \& Datt, J. Econ. Taxon. Bot. 34 (1): 66. 2010.

Habit: Herb.

Specimens examined: 31.viii.2003, Fakirpuri village, coll. S.D. Maliya 214876 (LWG).

Cyperus haspan L., Sp. PI. 1: 45. 1753.

C. halpan L., Sp. PI. 45. 1753; Maliya, J. Econ. Taxon. Bot. 36 (1): 161. 2012 \& 36 (2): 424. 2012.

Habit: Herb.

Specimens examined: Not observed. Record based on Maliya \& Datt (2012).

Cyperus iria L., Sp. PI. 45. 1753; Clarke in Hook. f., FI. Brit. India 6: 606. 1893; Duthie, Fl. Upp. Gang. Plain 3:
385. repr. ed. 1960 (excl. var. rectangularis); Saini, J. Econ. Taxon. Bot. 29(6): 896. 2005.

Habit: Herb.

Specimens examined: Not observed. Record based on Saini (2005).

Cyperus niveus Retz., Observ. Bot. 5: 12. 1788; Clarke in Hook. f., Fl. Brit. India 6: 601. 1893; Duthie, Fl. Upp. Gang. Plain 3: 383. repr. ed. 1960; Maliya \& Datt, J. Econ. Taxon. Bot. 34 (1): 66. 2010.

Habit: Herb.

Specimens examined: 17.iv.2003, Katerniaghat range, coll. S.D. Maliya 224918 (LWG).

Cyperus rotundus L., Sp. Pl. 45. 1753; Clarke in Hook. f., Fl. Brit. India 6: 614. 1893; Duthie, Fl. Upp. Gang. Plain 3: 388. repr. ed. 1960; Panigrahi et al., Bull. Bot. Surv. India 11 (1 \& 2): 111. 1969; Saini, J. Econ. Taxon. Bot. 29 (4): 897. 2005; Maliya \& Datt, J. Econ. Taxon. Bot. 34 (1): 66. 2010.

Habit: Herb.

Specimens examined: 08.ii.1965, Motipur range, O.P. Mishra 7718 (BSA).

Eleocharis acutangula (Roxb.) Schult. in Roem. \& Schult., Mant. 2: 91. 1824; Raizada, Suppl. Fl. Upp. Gang. Plain 306. 1976; Saini, J. Econ. Taxon. Bot. 29 (4): 898. 2005.

Scirpus acutangulus Roxb., Fl. Ind. 1: 216. 1820.

Habit: Herb.

Specimens examined: Not observed. Record based on Saini (2005).

Eleocharis dulcis (Burm. f.) Trin. ex Hensch., Vita Rumphii 186. 1833; Maliya \& Datt, J. Econ. Taxon. Bot. 34 (1): 66. 2010

Andropogon dulce Burm. f., FI. Ind. 219. 1768. Eleocharis equisetina Presl., Reliq. Haenk. 1: 195. 1828; Clarke in Hook. f., Fl. Brit. India 6: 626. 1893.

Habit: Herb.

Specimens examined: 31.viii.2003, Fakirpuri village, coll. S.D. Maliya 214874 (LWG); 05.iii.2011, Ghumna Bharu village, coll. S.D. Maliya 214925 (LWG).

**Eleocharis geniculata (L.) Roem. \& Schult., Syst. Veg. 2: 150. 1817; Saini, J. Econ. Taxon. Bot. 29 (4): 899. 2005.

Scirpus geniculatus L., Sp. PI. 48. 1753. Eleocharis capitata (L.) R. Br., Prodr. Fl. Nov. Holl. 225. 1810; Clarke in Hook. f., Fl. Brit. India 6: 627. 1893; Panigrahi et al., Bull. Bot. Surv. India 11 (1 \& 2): 111. 1969. 
Habit: Herb.

Specimens examined: 22.xi.1964, Orla, G. Panigrahi \& O.P. Mishra 6555 (BSA).

**Eleocharis palustris (L.) Roem. \& Schult., Syst. Veg. 2: 151.1817.

Scirpus palustris L., Sp. PI. 47. 1753. Eleocharis palustris (L.) R. Br., Prodr. 224. 1810, nom. illeg.; Clarke in Hook. f., FI. Brit. India 6: 628. 1893; Panigrahi et al., Bull. Bot. Surv. India 11 (1 \& 2): 111. 1969.

Habit: Herb.

Specimens examined: 12.iii.1964, Murtiha gate, G. Panigrahi 2886 (BSA); 09.ii.1965, Jairampurwa, O.P. Mishra 7760 (BSA).

**Fimbristylis dichotoma (L.) Vahl, Enum. PI. 2: 287. 1805; Duthie, Fl. Upp. Gang. Plain 3: 403. repr. ed. 1960; Maliya \& Datt, J. Econ. Taxon. Bot. 34 (1): 66. 2010.

Scirpus dichotomus L., Sp. PI. 50. 1753.

Habit: Herb.

Specimens examined: 23.viii.2002, Nishangara range, coll. S.D. Maliya 223619 (LWG); 10.xi.2002, Murtiha range, coll. S.D. Maliya 224570 (LWG).

Fimbristylis littoralis Gaudich., Voy. Uranie. 413. 1829; Saini, J. Econ. Taxon. Bot. 29 (4): 900. 2005.

Habit: Herb.

Specimens examined: Not observed. Record based on Saini (2005).

Fimbristylis ovata (Burm. f.) Kern, Blumea 15 (1): 126. 1967; Panigrahi et al., Bull. Bot. Surv. India 11 (1 \& 2): 111. 1969; Saini, J. Econ. Taxon. Bot. 29 (4): 901. 2005.

Carex ovata Burm. f., FI. Ind. 194. 1768. Fimbristylis monostachya (L.) Hassk., Pl. Jav. Rar. 61. 1848; Clarke in Hook. f., Fl. Brit. India 6: 649. 1893; Duthie, Fl. Upp. Gang. Plain 3: 405. repr. ed. 1960.

Habit: Herb.

Specimens examined: Not observed. Record based on Saini (2005).

Fimbristylis quinquangularis (Vahl) Kunth, Enum. PI. 2: 229. 1837; Clarke in Hook. f., Fl. Brit. India 6: 644. 1893; Duthie, FI. Upp. Gang. Plain 3: 406. repr. ed. 1960; Panigrahi et al., Bull. Bot. Surv. India 11 (1 \& 2): 111. 1969.

Scirpus quinquangularis Vahl, Enum. PI. 2: 279. 1805.

Habit: Herb.

Specimens examined: 20.xi.1964, Nishangara range, G. Panigrahi \& O.P. Mishra 6453 (BSA); 24.xi.1964, Nishangara range, G. Panigrahi \& O.P. Mishra 6598 (BSA).
Fimbristylis rigidula Nees in Wight, Contr. Bot. India 99. 1834; Duthie, Fl. Upp. Gang. Plain 3: 404. repr. ed. 1960; Maliya \& Datt, J. Econ. Taxon. Bot. 34 (1): 66. 2010.

Habit: Herb.

Specimens examined: 24.viii.2002, Nishangara range, coll. S.D. Maliya 223693 (LWG); 25.viii.2002, Murtiha range, coll. S.D. Maliya 223696 (LWG).

Kyllinga brevifolia Rottb., Descr. Icon. Rar. PI. 13, t. 4, f. 3. 1773; Duthie, Fl. Upp. Gang. Plain 3: 396. repr. ed. 1960; Maliya \& Datt, J. Econ. Taxon. Bot. 34 (1): 66.2010.

Cyperus brevifolia (Rottb.) Endl. ex Hassk., Cat. Hort. Bot. Bogor. 24. 1844; Panigrahi et al., Bull. Bot. Surv. India 11 (1 \& 2): 110. 1969; Saini, J. Econ. Taxon. Bot. 29 (4): 893. 2005.

Habit: Herb.

Specimens examined: 08.ii.1965, Motipur range, O.P. Mishra 7720 (BSA); 23.viii.2002, Nishangara range, coll. S.D. Maliya 223618 (LWG); 25.viii.2002, Murtiha range, coll. S.D. Maliya 223705 (LWG); 26.viii.2002, Katerniaghat range, coll. S.D. Maliya 223780 (LWG).

Pycreus flavidus (Retz.) Koyama, J. Jap. Bot. 51: 316. 1976; Maliya \& Datt, J. Econ. Taxon. Bot. 34 (1): 66. 2010.

Cyperus flavidus Retz., Observ. Bot. 5: 13. 1788; Duthie, Fl. Upp. Gang. Plain 3: 382. repr. ed. 1960; Saini, J. Econ. Taxon. Bot. 29 (6): 896. 2005.

Habit: Herb.

Specimens examined: 10.xi.2002, Murtiha range, coll. S.D. Maliya 224571 (LWG).

Pycreus pumilus (L.) Nees, Linnaea 9: 283. 1834; Maliya \& Datt, J. Econ. Taxon. Bot. 34 (1): 66. 2010.

Cyperus pumilus L., Cent. PI. 2: 6. 1756.

Habit: Herb.

Specimens examined: 29.x.2004, Kuthiyaghat forest, coll. S.D. Maliya 227138 (LWG).

Pycreus stramineus Clarke in Hook. f., Fl. Brit. India 6: 589. 1893; Maliya \& Datt, J. Econ. Taxon. Bot. 34 (1): 66. 2010.

Habit: Herb.

Specimens examined: 23.viii.2002, Nishangara range, coll. S.D. Maliya 223614 (LWG).

**Schoenoplectiella mucronata (L.) Jung \& Choi, J. PI. Biol. 53 (3): 230. 2010.

Scirpus mucronatus L., Sp. PI. 50. 1753; Clarke in Hook. f., FI. Brit. India 6: 657. 1893; Duthie, Fl. Upp. Gang. Plain 3: 409. repr. ed. 1960; Saini, J. Econ. Taxon. Bot. 29 (4). 903. 2005; Panigrahi et al., Bull. Bot. Surv. India 11 (1 \& 
2): 111. 1969. Schoenoplectus mucronatus (L.) Palla, Bot. Jahrb. Syst. 10: 299. 1888; Maliya \& Datt, J. Econ. Taxon. Bot. 66 (1): 57. 2010.

Habit: Herb.

Specimens examined: 26.v.1954, Motipur range, Hira Lal 13436 (LWG); 22.iv.2003, Nishangara range, coll. S.D. Maliya 224976 (LWG); Harraya village, coll. S.D. Maliya 214903 (LWG).

**Schoenoplectiella supina (L.) Lye, Lidia 6 (1): 27. 2003.

Scirpus supinus L., Sp. PI. 49. 1753; Panigrahi et al., Bull. Bot. Surv. India 11 (1 \& 2): 111. 1969. Schoenoplectus supinus (L.) Palla, Bot. Jahrb. Syst. 10: 299. 1888.

Habit: Herb.

Specimens examined: 22.xi.1964, Motipur on the way to Orla, G. Panigrahi \& O.P. Mishra 6551 (BSA).

**Scleria levis Retz., Observ. Bot. 4: 13. 1786; Clarke in Hook. f., Fl. Brit. India 6: 694. 1894; Saini, J. Econ. Taxon. Bot. 29 (4): 903. 2005.

Habit: Herb.

Specimens examined: Not observed. Record based on Saini (2005)

\section{DIOSCOREACEAE}

Dioscorea belophylla (Prain) Voigt. ex Haines, For. Fl. Chhota Nagpur 530. 1910; Duthie, Fl. Upp. Gang. Plain 3: 325. repr. ed. 1960; Maliya \& Datt, J. Econ. Taxon. Bot. 34 (1): 64. 2010; Saini, J. Econ. Taxon. Bot. 29 (4): 883. 2005.

D. nummularia var. belophylla Prain, Bengal PI. 2: 1065. 1903.

Habit: Climber.

Specimens examined: 05.ix.2005, Girijapuri forest, coll. S.D. Maliya 227151 (LWG).

Dioscorea bulbifera L., Sp. PI. 1033. 1753; Duthie, FI. Upp. Gang. Plain 3: 324. repr. ed. 1960; Saini, J. Econ. Taxon. Bot. 29 (4): 883. 2005; Maliya \& Datt, J. Econ. Taxon. Bot. 34 (1): 65. 2010.

D. bulbifera L. var. vera Prain \& Burkill, J. Proc. Asiat. Soc. Bengal 10: 26. 1914; Panigrahi et al., Bull. Bot. Surv. India 11 (1 \& 2): 110. 1969.

Habit: Climber.

Specimens examined: 25.xi.1964, Plantation area, Katerniaghat range, G. Panigrahi \& O.P. Mishra 6611 (BSA); 25.viii.2002, Murtiha range, coll. S.D. Maliya 223717 (LWG); 22.xii.2005, road side, Nishangara range, coll. S.D. Maliya 227179 (LWG); 24.x.2010, Nishangara range, coll. S.D. Maliya 252632 (LWG).
Dioscorea pubera Bl., Enum. PI. Javae 1: 21. 1827.

D. anguina Roxb., Fl. Ind. 3: 803. 1832; Duthie, Fl. Upp. Gang. Plain 3: 325. repr. ed. 1960; Panigrahi et al., Bull. Bot. Surv. India 11 (1 \& 2): 110.1969.

Habit: Climber.

Specimens examined: 12.iii.1964, Murtiha gate, G. Panigrahi \& O.P. Mishra 2891 (BSA); 07.xii.1986, Motipur range, K.K. Khanna \& R. Saran 38564 (BSA); 09.x.2009, near railway line, Nishangara range, coll. S.D. Maliya 226664 (LWG).

Dioscorea wightii Hook. f., Fl. Brit. India 6: 291. 1892; Panigrahi et al., Bull. Bot. Surv. India 11 (1 \& 2): 110. 1969.

Habit: Climber.

Specimens examined: 13.ii.1965, Urai tal, Bichhiya, O.P. Mishra 7980 (BSA).

\section{HYDROCHARITACEAE}

Blyxa aubertii Rich., Mem. Cl. Sci. Math. Inst. Natl. France 1811 (2): 19. 1814; Panigrahi et al., Bull. Bot. Surv. India 11 (1 \& 2): 109. 1969.

Habit: Herb.

Specimens examined: 23.xi.1964, about $3 \mathrm{~km}$. from Motipur (on the way to Kheri), G. Panigrahi \& O.P. Mishra 6589 (BSA).

**Hydrilla verticillata (L. f.) Royle, III. Bot. Himal. Mts. 1(10): 376. 1839; Maliya, J. Econ. Taxon. Bot. 36 (1): 159. 2012.

Serpicula verticillata L. f., Suppl. PI. 416. 1782.

Habit: Aquatic herb.

Specimens examined: Not observed. Record based on Maliya \& Datt (2012).

Najas graminea Delile, Descr. Egypte Hist. Nat. 2: 282, t. 50, f. 3. 1813; Hook. f. in Hook. f., Fl. Brit. India 6: 569. 1893; Duthie, Fl. Upp. Gang. Plain 3: 377. repr. ed. 1960; Panigrahi et al., Bull. Bot. Surv. India 11 (1 \& 2): 109.1969.

Habit: Herb.

Specimens examined: Not observed. Record based on Panigrahi et al. (1969).

Nechamandra alternifolia (Roxb.) Thw., Enum. PI. Zeyl. 332. 1864; Panigrahi et al., Bull. Bot. Surv. India 11 (1 \& 2): 109. 1969.

Vallisneria alternifolia Roxb., FI. Ind. 3: 750. 1832.

Habit: Herb.

Specimens examined: 22.xi.1964, Motipur on the way to Orla, G. Panigrahi \& O.P. Mishra 6544 (BSA). 
Ottelia alismoides (L.) Pers., Syn. PI. 1: 400. 1805; Hook. f. in Hook. f., Fl. Brit. India 5: 662. 1888; Duthie, FI. Upp. Gang. Plain 3: 265. repr. ed. 1960; Panigrahi et al., Bull. Bot. Surv. India 11 (1 \& 2): 109. 1969.

Stratiotes alismoides L., Sp. PI. 1: 535. 1753.

Habit: Aquatic herb.

Specimens examined: 12.iii.1964, Motipur nursery, G. Panigrahi 2849 (BSA); 21.xi.1964, Motipur on the way to Orla, G. Panigrahi \& O.P. Mishra 6657 (BSA); 22.xi.1964, Motipur on the way to Orla, G. Panigrahi \& O.P. Mishra 6534 (BSA).

Vallisneria spiralis L., Sp. PI. 1015. 1753; Hook. f. in Hook. f., Fl. Brit. India 5: 660. 1888; Duthie, Fl. Upp. Gang. Plain 3: 263. repr. ed. 1960; Subr. Aq. Ang. 57; Panigrahi et al., Bull. Bot. Surv. India 11 (1 \& 2): 109. 1969.

Habit: Aquatic herb.

Specimens examined: 12.iii.1964, Motipur range, G. Panigrahi 2848 (BSA); 20.xi.1964, Nishangara range, G. Panigrahi \& O.P. Mishra 6461 (BSA); 09.ii.1965, Motipur range, O.P. Mishra 7769 (BSA).

\section{HYPOXIDACEAE}

Curculigo orchioides Gaertn., Fruct. Sem. PI. 1: 63, t. 16, f. 11. 1788; Hook. f. in Hook. f., Fl. Brit. India 6: 279. 1892; Duthie, Fl. Upp. Gang. Plain 3: 319. repr. ed. 1960; Maliya \& Datt, J. Econ. Taxon. Bot. 34 (1): 64. 2010.

Habit: Herb.

Specimens examined: 21.v.1990, Murtiha range, K.K. Khanna 39921 (BSA); 25.xii.2003, Nishangara range, coll. S.D. Maliya 225269 (LWG); 22.iii.2004, Nishangara range, coll. S.D. Maliya 225935 (LWG); 19.vii.2004, Kuthiyaghat forest, coll. S.D. Maliya 225961 (LWG); 09.x.2009, Nishangara range, coll. S.D. Maliya 226665 (LWG); 28.ii.2011, Nishangara range, coll. S.D. Maliya 214913 (LWG).

\section{LILIACEAE}

Lilium polyphyllum D. Don in Royle III. Bot. Himal. Mts. 1 (11): 388. 1840; Maliya \& Datt, J. Econ. Taxon. Bot. 34 (1): 65.2010.

Habit: Herb.

Specimens examined: 25.xii.2003, mid point of Katerniaghat \& Nishangara range, coll. S.D. Maliya 225263 (LWG).

\section{MUSACEAE}

*Musa balbisiana Colla, Mem. Reale. Accad. Sci. Torino 25: 384. 1820; Maliya \& Datt, J. Econ. Taxon. Bot. 34 (1): 64. 2010.

Habit: Tree like herb, cultivated.
Specimens examined: Not observed. Record based on Maliya \& Datt (2010).

\section{ORCHIDACEAE}

Calanthe plantaginea Lindl., (in Wall. Cat. 7346. 1830, nom. nud.) Gen. Sp. Orchid. PI. 250. 1833; Hook. f. in Hook. f., Fl. Brit. India 5: 853. 1890; Maliya \& Datt, J. Econ. Taxon. Bot. 34 (1): 64. 2010.

Habit: Herb.

Specimens examined: Not observed. Record based on Maliya \& Datt (2010).

Eulophia dabia (D. Don) Hochr., Bull. New York Bot. Gard. 6: 270. 1910; Santapau \& Kapadia, Orch. Bomb. 112. 1966; Saini, J. Econ. Taxon. Bot. 29 (4): 881. 2005.

Bletia dabia D. Don, Prodr. Fl. Nepal. 30. 1825. Eulophia campestris Wall. ex Lindl., Gen. Sp. Orchid. PI. 185. 1833; Duthie, Fl. Upp. Gang. Plain 3: 281. repr. ed. 1960.

Habit: Herb.

Specimens examined: Not observed. Record based on Saini (2005).

Habenaria digitata Lindl., Gen. Sp. Orchid. PI. 307. 1835; Maliya, J. Econ. Taxon. Bot. 36 (2): 423.2012.

Habit: Herb.

Specimens examined: 24.x.2010, Nishangara range, coll. S.D. Maliya 252631 (LWG).

Habenaria plantaginea (Lindl.) Lindl., Gen. Sp. Orchid. PI. 323. 1835; Duthie, Fl. Upp. Gang. Plain 3: 301. repr. ed. 1960; Panigrahi et al., Bull. Bot. Surv. India 11 (1 \& 2): 110. 1969.

Gymnadenia plantaginea Lindl., Numer. List n. 7053. 1828.

Habit: Herb.

Specimens examined: 24.xi.1964, Nishangara range, G. Panigrahi \& O.P. Mishra 6591 (BSA); 07.x.2009, Kakraha rest house, Kakraha range, coll. S.D. Maliya 226650 (LWG).

Nervilia aragoana Gaudich., Voy. Uranie 422, t. 35. 1829; Maliya \& Datt, J. Econ. Taxon. Bot. 34 (1): 64.2010. Habit: Herb.

Specimens examined: Not observed. Record based on Maliya \& Datt (2010).

Peristylus affinis (D. Don) Seidenf., Dansk Bot. Ark. 31 (3): 48, f. 23. 1977; Maliya \& Datt, J. Econ. Taxon. Bot. 34

(1): 64.2010.

Habenaria affinis D. Don, Prodr. Fl. Nepal. 25. 1825. Habit: Herb. 
Specimens examined: Not observed. Record based on Maliya \& Datt (2010).

Peristylus constrictus (Lindl.) Lindl., Gen. Sp. Orchid. PI. 300 1835; Maliya, J. Econ. Taxon. Bot. 36 (2): 423.2012.

Herminium constrictum Lindl. Edwards's Bot. Reg. sub pl. 1499. 1832.

Habit: Herb.

Specimens examined: 07.xi.2008, Kuthiyaghat forest, coll. S.D. Maliya 226649 (LWG).

Vanda tessellata (Roxb.) Hook. f. ex G. Don in J. C. Loudon, Hort. Brit. 372. 1830; Maliya \& Datt, J. Econ. Taxon. Bot. 35 (1): 37.2011.

Epidendrum tessellatum Roxb., Pl. Coromandel 1: 34. 1795.

Habit: Epiphytes with leafy stem.

Specimens examined: 10.x.2009, Katerniaghat range, coll. S.D. Maliya 226669 (LWG).

Zeuxine strateumatica (L.) Schltr., Bot. Jahrb. Syst. 45 (3): 394. 1911; Panigrahi et al., Bull. Bot. Surv. India 11 (1 \& 2): 110. 1969; Maliya \& Datt, J. Econ. Taxon. Bot. 34 (1): 64. 2010

Orchis strateumatica L., Sp. PI. 943. 1753.

Habit: Herb.

Specimens examined: Not observed. Record based on Panigrahi et al. (1969) and Maliya \& Datt (2010).

\section{POACEAE}

Alopecurus nepalensis Trin. ex Steud., Syn. Pl. Glumac. 1: 148. 1854; Hook. f. in Hook. f., Fl. Brit. India 7: 239. 1897; Saini, J. Econ. Taxon. Bot. 29 (4): 904. 2005.

Habit: Annual grass.

Specimens examined: Not observed. Record based on Saini (2005).

Apluda mutica L., Sp. PI. 82. 1753; Panigrahi et al., Bull. Bot. Surv. India 11 (1 \& 2): 111. 1969; Maliya \& Datt, J. Econ. Taxon. Bot. 34 (1): 66. 2010

Habit: Perennial grass.

Specimens examined: 20.xi.1964, rest house, Nishangara range, G. Panigrahi \& O.P. Mishra 6498 (BSA); 16.ii.1965, Katerniaghat mandir, O.P. Mishra 8026 (BSA); 09.xi.2002, road side, Katerniaghat range, coll. S.D. Maliya 224560 (LWG); 12.xi.2002, Nishangara range, coll. S.D. Maliya 224565 (LWG); 23.x.2010, Nishangara range, coll. S.D. Maliya 252623 (LWG).

Arundinella bengalensis (Spreng.) Druce, Rep. Bot Soc. Exch. Club Brit. Isles 4: 605. 1917; Panigrahi et al.,
Bull. Bot. Surv. India 11 (1 \& 2): 111. 1969.

Panicum bengalense Spreng., Syst. Veg. 1: 311. 1825.

Habit: Grass.

Specimens examined: 24.xi.1964, Nishangara range, G. Panigrahi \& O.P. Mishra 6593 (BSA).

**Arundo donax L., Sp. PI. 81. 1753; Panigrahi et al., Bull. Bot. Surv. India 11 (1 \& 2): 111. 1969; Saini, J. Econ. Taxon. Bot. 29 (4): 904. 2005; Maliya \& Datt, J. Econ. Taxon. Bot. 34 (1): 66. 2010.

Habit: Grass.

Specimens examined: Not observed. Record based on Panigrahi et al. (1969), Saini (2005) and Maliya \& Datt (2010).

**Avena sativa L., Sp. PI. 79. 1753; Saini, J. Econ. Taxon. Bot. 29 (4): 905. 2005; Maliya \& Datt, J. Econ. Taxon. Bot. 34 (1): 67. 2010.

Habit: Grass, occassinaly found wheat field.

Specimens examined: 20.iii.2004, Girijapuri forest, coll. S.D. Maliya 225929 (LWG).

Bambusa bambos (L.) Voss, Vilm. Blumengartn. 1: 1189. 1895.

Arundo bambos L., Sp. PI. 81. 1753. Bambos arundinacea Retz., Observ. Bot. 5: 24. 1789. Bambusa arundinacea (Retz.) Willd., Sp. Pl. 2 (1): 245. 1799; Saini, J. Econ. Taxon. Bot. 29 (4): 905. 2005.

Habit: Tree like shrub.

Specimens examined: Not observed. Record based on Saini (2005).

**Bothriochloa bladhii (Retz.) Blake, Proc. Roy. Soc. Queensland 80 (6): 62. 1969.

Andropogon bladhii Retz., Observ. Bot. 2: 27. 1781. A. intermedius R. Br., Prodr. Fl. Nov. Holl. 1: 202. 1810. A. glaber Roxb., Fl. Ind. 1: 271. 1820. Bothriochloa intermedia (R. Br.) Camus, Ann. Soc. Linn. Lyonn.s. 76: 164. 1931; Panigrahi et al., Bull. Bot. Surv. India 11 (1 \& 2): 111. 1969. B. glabra (Roxb.) Camus, Ann. Soc. Linn. Lyonn.s. 76: 164. 1931; Panigrahi et al., Bull. Bot. Surv. India 11 (1 \& 2): 111. 1969.

Habit: Grass.

Specimens examined: 20.xi.1964, Nishangara range, G. panigrahi \& O.P. Mishra 6445 (BSA). 10.ii.1965, Nishangara range, O.P. Mishra 7902 (BSA).

Brachiaria subquadripara (Trin.) Hitchc., Lingnan Sci. J. 7: 214. 1931; Panigrahi et al., Bull. Bot. Surv. India 11 (1 \& 2): 111. 1969.

Panicum subquadriparum Trin., Gram. Panic. 145. 
1826.

Habit: Grass.

Specimens examined: 24.xi.1964, Nishangara range, G. Panigrahi \& O.P. Mishra 6600 (BSA).

Chionachne gigantea (Koenig) Veldkamp, Blumea 47 (3): 559, f. 3. 2002.

Coix gigantea Koenig, Naturforscher (Halle) 23: 211. 1788. C. koenigii Spreng., Syst. Veg. 1: 239. 1824. Chionachne koenigii (Spreng.) Thw., Enum. PI. Zeyl. 357. 1864; Panigrahi et al., Bull. Bot. Surv. India 11 (1 \& 2): 111. 1969.

Habit: Perennial grass.

Specimens examined: 15.ii.1964, Rampurwa, G. Panigrahi 2918 (BSA); 20.xi.1964, Nishangara range, G. Panigrahi \& O.P. Mishra 6441 (BSA); 11.ii.1965, Bichhiya, Nishangara range, O.P. Mishra 7951 (BSA).

Chrysopogon aciculatus (Retz.) Trin., Fund. Agrost. 188. 1820; Saini, J. Econ. Taxon. Bot. 29 (4): 906. 2005; Panigrahi et al., Bull. Bot. Surv. India 11 (1 \& 2): 111.1969.

Andropogon aciculatus Retz., Observ. Bot. 5: 22. 1789. Habit: Grass.

Specimens examined: 22.xi.1964, Orla, Motipur, G. Panigrahi \& O.P. Mishra 6578 (BSA).

Chrysopogon gryllus (L.) Trin., Fund. Agrost. 188. 1820; Maliya \& Datt, J. Econ. Taxon. Bot. 34 (1): 67. 2010.

Andropogon gryllus L., Cent. PI. 2: 33. 1756.

Habit: Grass.

Specimens examined: 25.viii.2002, Murtiha range, coll. S.D. Maliya 223736 (LWG); 31.viii.2003, Fakirpuri village, coll. S.D. Maliya 214884 (LWG).

Chrysopogon serrulatus Trin., Mem. Acad. Imp. Sci. St. - Petersbourg, Ser. 6, Sci. Math. 2 (3): 318. 1833; Maliya \& Datt, J. Econ. Taxon. Bot. 34 (1): 67. 2010.

Habit: Grass.

Specimens examined: 12.xi.2002, Nishangara range, coll. S.D. Maliya 224655 (LWG).

Chrysopogon zizanioides (L.) Roberty, Bull. Inst. Franc. Afrique Noire, A 22: 106. 1960.

Vetiveria zizanioides (L.) Nash in Small, FI. S. E. U. S. 67 1326. 1903; Panigrahi et al., Bull. Bot. Surv. India 11 (1 \& 2): 113. 1969; Maliya \& Datt, J. Econ. Taxon. Bot. 34 (1): 68. 2010. Phalaris zizanioides L., Mant. PI. 2: 183. 1771.

Habit: Grass.

Specimens examined: 13.ii.1965, Nishangara range, O.P. Mishra 7979 (BSA); 20.xi.1964, Nishangara range, G. Panigrahi \& O.P. Mishra 6471 (BSA); 23.viii.2002,
Nishangara range, coll. S.D. Maliya 223617 (LWG); 24.viii.2002, Nishangara range, coll. S.D. Maliya 223691 (LWG); 26.viii.2002, Katerniaghat range, coll. S.D. Maliya 223757 (LWG).

Coix lacryma-jobi L., Sp. PI. 972. 1753; Hook. f. in Hook. f., Fl. Brit. India 7: 100. 1897; Panigrahi et al., Bull. Bot. Surv. India 11 (1 \& 2): 111. 1969.

Habit: Grass.

Specimens examined: 11.ii.1965, Bichhiya, Nishangara range, O.P. Mishra 7956 (BSA); 25.x.2010, near Fakirpuri village, Rampurwa forest, coll. S.D. Maliya 252633 (LWG).

**Cymbopogon jwarancusa (Jones) Schult. ssp. olivieri (Boiss.) Soenarko, Reinwardtia 9 (3): 307. 1977; Maliya \& Datt, J. Econ. Taxon. Bot. 34 (1): 67. 2010.

Andropogon olivieri Boiss., Diagn. Pl. Orient. 5: 76. 1844.

Habit: Grass.

Specimens examined: 17.iv.2003, Katerniaghat range, coll. S.D. Maliya 224923 (LWG)

Cymbopogon martini (Roxb.) Watson in Atkinson, Gaz. N.-W. Prov. India 10: 392. 1882; Panigrahi et al., Bull. Bot. Surv. India 11 (1 \& 2): 111. 1969.

Andropogon martini Roxb., (Hort. Bengal. 7. 1814) Fl. Ind. 1: 280. 1820.

Habit: Grass.

Specimens examined: 12.xi.1964, Nishangara range, G. Panigrahi \& O.P. Mishra 6470 (BSA); 11.ii.1965, Bichhiya, Nishangara range, O.P. Mishra 7963 (BSA).

**Cymbopogon microstachys (Hook,f.) Soenarko, Reinwardtia 9: 364. 1977.

Andropogon nardus var. microstachys Hook. f., Fl. Brit. India 7 (21): 207. 1896. Cymbopogon flexuosus (Nees ex Steud.) Wats. var. microstachys (Hook. f.) Bor, J. Bombay Nat. Hist. Soc. 52: 162. 1954.

Habit: Grass.

Specimens examined: 12.iii.1964, Murtiha range, G. Panigrahi 2858 (BSA); 20.xi.1964, Nishangara range, O.P. Mishra \& G. Panigrahi 6435 (BSA); 16.ii.1965, Katerniaghat range, O.P. Mishra 8025 (LWG).

Cynodon dactylon (L.) Pers., Syn. PI. 1: 85. 1805; Saini, J. Econ. Taxon. Bot. 29 (4): 907. 2005; Maliya \& Datt, J. Econ. Taxon. Bot. 34 (1): 67. 2010.

Panicum dactylon L., Sp. PI. 58. 1753.

Habit: Grass.

Specimens examined: 13.xi.2011. Kakraha range, A. Kumar \& O. Bajpai 252790 (LWG). 
Cynodon radiatus Roth. in Roem. \& Schult., Syst. Veg. 2: 411.1817.

C. arcuatus Presl., Reliq. Haenk. 1(4-5): 290. 1830; Maliya \& Datt, J. Econ. Taxon. Bot. 34 (1): 67. 2010.

Habit: Grass.

Specimens examined: 25.viii.2002, Murtiha range, coll. S.D. Maliya 223699 (LWG).

Cyrtococcum patens (L.) Camus, Bull. Mus. Natl. Hist. Nat. 27 (1): 118. 1921; Panigrahi et al., Bull. Bot. Surv. India 11 (1 \& 2): 112. 1969.

Panicum patens L., Sp. PI. 58. 1753; Hook. f. in Hook. f., Fl. Brit. India 7: 57. 1897.

Habit: Creeping grass.

Specimens examined: 10.ii.1965, Nishangara range, O.P. Mishra 7904 (BSA).

**Dactyloctenium aegyptium (L.) Willd., Enum. PI. 2: 1029. 1809.

Cyanosurus aegyptius L., Sp. Pl. 72. 1753. Dactyloctenium aegyptium (L.) Beauv., Ess. Agrost. Expl. PI. 15. 1818; Panigrahi et al., Bull. Bot. Surv. India 11 (1 \& 2): 112. 1969; Saini, J. Econ. Taxon. Bot. 29 (4): 907.2005. Habit: Grass.

Specimens examined: 22.xi.1964, Orla, Motipur, G. Panigrahi \& O.P. Mishra 6583 (BSA); 12.xii.1986, Motipur, K.K. Khanna \& R. Saran 38666 (BSA).

Dendrocalamus strictus (Roxb.) Nees, Linnaea 9 (4): 476. 1835; Gamble in Hook. f., Fl. Brit. India7: 404. 1897; Maliya \& Datt, J. Econ. Taxon. Bot. 34 (1): 67. 2010.

Bambos stricta Roxb., PI. Coromandel 1: 58, t. 80. 1798.

Habit: Grass.

Specimens examined: 21.v.1987, K.K. Singh 5986 (LWG); 25.viii.2002, Murtiha range, coll. S.D. Maliya 223739 (LWG); 07.ix.2005, Kailashpuri forest, coll. S.D. Maliya 227157 (LWG).

Desmostachya bipinnata (L.) Stapf., Fl. Cap. 7: 632. 1900; Maliya \& Datt, J. Econ. Taxon. Bot. 34 (1): 67.2010.

Briza bipinnata L., Fl. Palaest. 12. 1756.

Habit: Grass.

Specimens examined: 24.viii.2002, Nishangara range, coll. S.D. Maliya 223690 (LWG); 25.viii.2002, murtiha range, coll. S.D. Maliya 223726 (LWG).

Dichanthium annulatum (Forssk.) Stapf. in Prain, Fl. Trop. Afr. 9: 178. 1917; Panigrahi et al., Bull. Bot. Surv. India 11 (1 \& 2): 112. 1969; Maliya \& Datt, J. Econ. Taxon. Bot. 34 (1): 67. 2010.
Andropogon annulatus Forssk., FI. Aegypt. - Arab. 173. 1775; Hook. f. in Hook. f., Fl. Brit. India 7: 196. 1897.

Habit: Grass.

Specimens examined: 09.ii.1965, Jairampurwa, O.P. Mishra 7758 (BSA); 10.ii.1965, Nishangara range, O.P. Mishra 7905 (BSA); 13.ii.1965, Nishangara range, O.P. Mishra 7977 (BSA); 10.xi.2002, Murtiha range, coll. S.D. Maliya 224573 (LWG).

Dichanthium foveolatum (Delile) Roberty, Monogr. Syst. Andropog. du Globe: 163. 1960 \& Monogr. Syst. Andropog. du Globe: 163. 1960.

Andropogon foveolatus Delile, Descr. Egypte, Hist. Nat. 2: 160. 1813. Eremopogon foveolatus (Delile) Stapf., Fl. Trop. Africa 9: 183. 1917; Panigrahi et al., Bull. Bot. Surv. India 11 (1 \& 2): 112. 1969.

Habit: Grass.

Specimens examined: 16.ii.1965, O.P. Mishra 8017 (BSA).

Digitaria bicornis (Lam.) Roem. \& Schult., Syst. Veg. 2: 470. 1817; Maliya \& Datt, J. Econ. Taxon. Bot. 34 (1): 67. 2010.

Paspalum bicorne Lam., Encycl. 1: 176. 1791.

Habit: Grass.

Specimens examined: 25.viii.2002, Murtiha range, coll. S.D. Maliya 223700 (LWG).

Digitaria longiflora (Retz.) Pers., Syn. Pl. 1: 85. 1805; Panigrahi et al., Bull. Bot. Surv. India 11 (1 \& 2): 112.1969.

Paspalum longiflorum Retz., Observ. Bot. 4: 15. 1786.

Habit: Grass.

Specimens examined: 16.ii.1965, Motipur range, G. Panigrahi \& O.P. Mishra 2842 (BSA).

Digitaria stricta Roth. in Roem. \& Schult., Syst. Veg. 2: 474.1817.

D. denudata Link, Hort. Berol. 1: 222. 1827. D. stricta Roth. in Roem. \& Schult. var. denudata (Link) Henrard, Monogr. Digitaria 175. 1950; Panigrahi et al., Bull. Bot. Surv. India 11 (1 \& 2): 112. 1969.

Habit: Grass.

Specimens examined: 20.xi.1964, Nishangara range, G. Panigrahi \& O.P. Mishra 6446 (BSA); 24.xi.1964, Nishangara range, G. Panigrahi \& O.P. Mishra 6605A (BSA).

Echinochloa colona (L.) Link, Hort. Berol. 2: 209. 1833; Panigrahi et al., Bull. Bot. Surv. India 11 (1 \& 2): 112. 1969; Saini, J. Econ. Taxon. Bot. 29 (4): 909. 2005; Maliya \& Datt, J. Econ. Taxon. Bot. 34 (1): 67. 2010. 
Panicum colonum L., Syst. Nat. 2: 870. 1759.

Habit: Grass.

Specimens examined: 11.iii.1964, Sujauli, G. Panigrahi 2801 (BSA); 08.ii.1965, Motipur range, O.P. Mishra 7743 (BSA).

Echinochloa crus-galli (L.) P. Beauv., Ess. Agrostogr. 1: 53, 161, 169, t. 11, f. 2. 1812.

Panicum crus-galli L., Sp. PI. 56. 1753; Hook. f. in Hook. f., Fl. Brit. India 7: 30.1897 (in part). Echinochloa crusgalli (L.) P. Beauv., Ess. Agrost. 53: 161. 1812; Saini, J. Econ. Taxon. Bot. 29 (4): 909. 2005.

Habit: Grass.

Specimens examined: Not observed. Record based on Saini (2005).

Eragrostis amabilis (L.) Wight \& Arn., Cat. Indian PI. 2: 105.1834.

Poa amabilis L., Sp. PI. 68. 1753. P. tenella L., Sp. PI. 69. 1753. Eragrostis tenella (L.) Roem. \& Schult., Syst. Veg. 2: 576. 1817; O. Stapf. in Hook. f., Fl. Brit. India 7: 315. 1897; Panigrahi et al., Bull. Bot. Surv. India 11 (1 \& 2): 112. 1969. Habit: Grass.

Specimens examined: 08.xii.1965, Motipur range, O.P. Mishra 7721 (BSA).

**Eragrostis atrovirens (Desf.) Trin ex Steud., Nomencl. Bot. 1: 562. 1840; Panigrahi et al., Bull. Bot. Surv. India 11 (1 \& 2): 112. 1969.

Poa atrovirens Desf., Fl. Atlant. 1: 73, t. 14. 1798.

Habit: Grass.

Specimens examined: 22.xi.1964, Motipur on the way to Orla, G. Panigrahi \& O.P. Mishra 6540 (BSA).

Eragrostis japonica (Thunb.) Trin., Mem. Acad. Imp. Sci. St. - Petersbourg, Ser. 6, Sci. Math. 1 (4): 405.1830.

Poa japonica Thunb., Fl. Jap. 51. 1784. P. diarrhena Schult. \& Schult. f. in Roem. \& Schult., Mant. 3: 616. 1827. Eragrostis diarrhena (Schult. \& Schult. f.) Steud., Syn. Pl. Glumac. 1: 268. 1854; Panigrahi et al., Bull. Bot. Surv. India 11 (1 \& 2): 112. 1969.

Habit: Grass.

Specimens examined: 09.ii.1965, Jairampurwa, O.P. Mishra 7765 (BSA); 22.x.2010, Nishangara range, coll. S.D. Maliya 252621 (LWG).

**Eragrostis unioloides (Retz.) Nees ex Steud., Syn. PI. Glumac. 1: 264. 1854; Panigrahi et al., Bull. Bot. Surv. India 11 (1 \& 2): 112. 1969; Saini, J. Econ. Taxon. Bot. 29 (4): 911.2005.

Poa unioloides Retz., Observ. Bot. 5: 19. 1789.
Habit: Grass.

Specimens examined: 24.xi.1964, Nishangara range, G. Panigrahi \& O.P. Mishra 6599 (BSA).

Eulaliopsis binata (Retz.) Hubb. in Hook., Icon. PI. 33 (3): t. 3262, p. 6. 1935; Panigrahi et al., Bull. Bot. Surv. India 11 (1 \& 2): 112. 1969.

Andropogon binatus Retz.,Observ. Bot. 5: 21. 1789.

Habit: Grass.

Specimens examined: 11.ii.1965, Bichhiya range, Nishangara range, O.P. Mishra 7964 (BSA).

Heteropogon contortus (L.) Beauv. ex Roem. \& Schult., Syst. Veg. 2: 836. 1817; Panigrahi et al., Bull. Bot. Surv. India 11 (1 \& 2): 112. 1969.

Andropogon contortus L., Sp. PI. 1045. 1753; Hook. f. in Hook. f., Fl. Brit. India 7: 199. 1897.

Habit: Grass.

Specimens examined: 08.ii.1965, Motipur range, O.P. Mishra 7748 (BSA); 12.ii.1965, Rampurwa, Nishangara range, O.P. Mishra 7949 (BSA).

Hygroryza aristata (Retz.) Nees ex Wight. \& Arn., Edinburgh New Philos. J. 15: 380. 1833; Maliya \& Datt, J. Econ. Taxon. Bot. 34 (1): 67. 2010.

Pharus aristatus Retz., Observ. Bot. 5: 23. 1789.

Habit: Grass.

Specimens examined: 22.x.2004, Kailashpuri forest, coll. S.D. Maliya 225999 (LWG).

Imperata cylindrica (L.) Raeusch., Nomencl. Bot., ed. 3: 10.1797.

Lagurus cylindricus L., Syst. Nat. 2: 878. 1759. Imperata cylindrica (L.) P. Beauv., Ess. Agrostogr. 8, 165, 177, t. 5, f. 1. 1812; Panigrahi et al., Bull. Bot. Surv. India 11 (1\& 2): 112. 1969. I. cylindrica (L.) P. Beauv. var. major (Nees) Hubb., Grass. Maur. Rod. 96. 1940; Panigrahi et al., Bull. Bot. Surv. India 11 (1 \& 2): 112. 1969. I. koenigii var. major Nees, Fl. Afr. Austral. III. 90. 1841.

Habit: Grass.

Specimens examined: 24.xi.1964, Nishangara range, G. Panigrahi \& O.P. Mishra 6602A (BSA).16.ii.1965, O.P. Mishra 8007 (BSA); 16.ii.1965, O.P. Mishra 8010 (BSA).

Isachne albens Trin., Sp. Gram. 1(8): t. 85. 1827; Hook. f. in Hook. f., Fl. Brit. India 7: 22. 1897; Saini, J. Econ. Taxon. Bot. 29 (4): 912. 2005.

Habit: Grass.

Specimens examined: Not observed. Record based on Saini (2005). 
Leptochloa panicea (Retz.) Ohwi, Bot. Mag. Tokyo 55 (655): 311. 1941; Saini, J. Econ. Taxon. Bot. 29 (4): 913. 2005.

Poa panicea Retz., Observ. Bot. 3: 11. 1783.

Habit: Grass.

Specimens examined: Not observed. Record based on Saini (2005)

Ophiuros exaltatus (L.) Kuntze, Revis. Gen. PI. 2: 780. 1891.

Aegilops exaltata L., Mant. PI. 2: 575. 1771. Rottboellia exaltata (L.) L. f., Nov. Gram. Gen. 40, t. 1. 1779; Maliya \& Datt, J. Econ. Taxon. Bot. 34 (1): 67. 2010.

Habit: Grass.

Specimens examined: 12.xi.2002, Nishangara range, coll. S.D. Maliya 224630 (LWG).

Oplismenus compositus (L.) P. Beauv., Ess. Agrost. 54. 1812; Hook. f. in Hook. f., Fl. Brit. India 7: 66. 1897; Panigrahi et al., Bull. Bot. Surv. India 11 (1 \& 2): 113. 1969; Saini, J. Econ. Taxon. Bot. 29 (4): 913. 2005; Maliya \& Datt, J. Econ. Taxon. Bot. 34 (1): 67. 2010.

Panicum compositum L., Sp. PI. 57. 1753.

Habit: Grass.

Specimens examined: 21.xi.1964, Nishangara range, G. Panigrahi \& O.P. Mishra 6501 (BSA); 09.xi.2002, Katerniaghat range, coll. S.D. Maliya 224559 (LWG); 10.xi.2002, Murtiha range, coll. S.D. Maliya 224572 (LWG).

Oryza rufipogon Griff., Not. PI. Asiat. 3: 5, t. 144, f. 2. 1851; Panigrahi et al., Bull. Bot. Surv. India 11 (1 \& 2): 113. 1969.

Habit: Grass.

Specimens examined: 23.xi.1964, Kharia nala, Motipur range, G. Panigrahi \& O.P. Mishra 6572 (BSA).

**Oryza sativa L., Sp. PI. 33. 1753; Panigrahi et al., Bull. Bot. Surv. India 11 (1 \& 2): 113. 1969.

Habit: Grass.

Specimens examined: 20.xi.1964, Orla, Motipur range, G. Panigrahi \& O.P. Mishra 6576 (BSA).

**Panicum maximum Jacq., Icon. PI. Rar. 1: 2, t. 13. 1781; Maliya \& Datt, J. Econ. Taxon. Bot. 34 (1): 67. 2010.

Megathyrsus maximus (Jacq.) Simon \& Jacobs, Austrobaileya 6: 572. 2003.

Habit: Grass.

Specimens examined: 23.viii.2002, Nishangara range, coll. S.D. Maliya 223620 (LWG).
Panicum paludosum Roxb., FI. Ind. 1: 310. 1820; Panigrahi et al., Bull. Bot. Surv. India 11 (1 \& 2): 113. 1969. Habit: Grass.

Specimens examined: 22.xi.1964, Orla, Motipur range, G. Panigrahi \& O.P. Mishra 6568 (BSA).

Panicum repens L., Sp. PI. 87. 1762; Maliya \& Datt, J. Econ. Taxon. Bot. 34 (1): 67. 2010.

Habit: Grass.

Specimens examined: 23.viii.2002, Nishangara range, coll. S.D. Maliya 223622 (LWG); 25.viii.2002, Nishangara range, coll. S.D. Maliya 223625 (LWG).

Paspalidium flavidum (Retz.) Camus in Lecomte, Fl. Indo-Chine 7: 419. 1922; Maliya \& Datt, J. Econ. Taxon. Bot. 34 (1): 67. 2010.

Panicum flavidum Retz., Observ. Bot. 4: 15. 1786; Hook. f. in Hook. f., Fl. Brit. India 7: 28. 1897.

Habit: Grass.

Specimens examined: 23.viii.2002, Nishangara range, coll. S.D. Maliya 223623 (LWG); 25.viii.2002, Murtiha range, coll. S.D. Maliya 223701 (LWG).

**Paspalum distichum L., Syst. Nat. 2: 855. 1759; Hook. f. in Hook. f., Fl. Brit. India 7: 12. 1897; Panigrahi et al., Bull. Bot. Surv. India 11 (1 \& 2): 113. 1969.

Habit: Grass.

Specimens examined: 24.xi.1964, Nishangara range, G. Panigrahi \& O.P. Mishra 6609 (BSA).

Pennisetum glaucum (L.) R. Br., Prodr. Fl. Nov. Holl. 195. 1810.

Panicum glaucum L., Sp. PI. 56. 1753. Setaria glauca (L.) Beauv., Ess. Agrost. 51, 169, 178. 1812; Panigrahi et al., Bull. Bot. Surv. India 11 (1 \& 2): 113. 1969.

Habit: Grass.

Specimens examined: 20.xi.1964, Nishangara range, G. Panigrahi \& O.P. Mishra 6447 (BSA).

Phacelurus speciosus (Steud.) Hubb., Bull. Misc. Inform. Kew 1928: 35. 1928; Panigrahi et al., Bull. Bot. Surv. India 11 (1 \& 2): 113. 1969.

Andropogon speciosus Steud., Syn. PI. Glumac. 1: 375. 1854.

Habit: Grass.

Specimens examined: 20.xi.1964, Nishangara range, G. Panigrahi \& O.P. Mishra 6466 (BSA).

Phalaris minor Retz., Observ. Bot. 3: 8. 1783; Hook. f. in Hook. f., Fl. Brit. India 7: 221. 1897; Maliya \& Datt, J. Econ. Taxon. Bot. 34 (1): 67. 2010. 
Habit: Grass.

Specimens examined: 20.iii.2004, Girijapuri forest, coll. S.D. Maliya 225925 (LWG).

**Phragmites vallatorius (L.) Veldk., Blumea 37 (1) 233. 1992; Maliya \& Datt, J. Econ. Taxon. Bot. 34 (1): 67. 2010.

Arundovallatoria L., Herb.Amb.15.1754.A. karka Retz., Observ. Bot. 4: 21. 1786. Phragmites karka (Retz.) Trin. ex Steud., Nomencl. Bot. 2: 324. 1841; Panigrahi et al., Bull. Bot. Surv. India 11 (1 \& 2): 113. 1969.

Habit: Grass.

Specimens examined: 20.xi.1964, Nishangara range, G. Panigrahi \& O.P. Mishra 6462 (BSA); 13.ii.1965, Nishangara range, O.P. Mishra 7968 (BSA); 22.iv.2003, Nishangara range, coll. S.D. Maliya 224988 (LWG); 21.xii.2003, Katerniaghat range, coll. S.D. Maliya 225222 (LWG); 05.xi.2008, Katerniaghat range, coll. S.D. Maliya 226634 (LWG); 01.iii.2011, Harayya village, coll. S.D. Maliya 214915 (LWG).

**Polypogon monspeliensis (L.) Desf., Fl. Atlant. 1: 67. 1798; Hook. f. in Hook. f., Fl. Brit. India 7: 245. 1897; Panigrahi et al., Bull. Bot. Surv. India 11 (1 \& 2): 113. 1969; Maliya \& Datt, J. Econ. Taxon. Bot. 34 (1): 67. 2010.

Alopecurus monsepeliensis L., Sp. PI. 61. 1753.

Habit: Grass.

Specimens examined: 11.iii.1964, Sujauli, G. Panigrahi 2800 (BSA); 22.iv.2003, Nishangara range, coll. S.D. Maliya 224968 (LWG).

Saccharum arundinaceum Retz., Observ. Bot. 4: 14. 1786; Panigrahi et al., Bull. Bot. Surv. India 11 (1 \& 2): 113. 1969.

Erianthus arundinaceus (Retz.) Jeswiet, Arch. Suikerindustr. Ned. Ned.-Inde 12: 399. 1925.

Habit: Grass.

Specimens examined: 08.ii.1965, Kauriala, O.P. Mishra 7751 (BSA); 11.ii.1965, Bichhiya, O.P. Mishra 7928 (BSA).

Saccharum bengalense Retz., Observ. Bot. 5: 16. 1789; Panigrahi et al., Bull. Bot. Surv. India 11 (1 \& 2): 113 1969; Saini, J. Econ. Taxon. Bot. 29 (4): 916. 2005; Maliya \& Datt, J. Econ. Taxon. Bot. 34 (1): 67. 2010.

S. munja Roxb., FI. Ind. 1: 250. 1820. Erianthus munja (Roxb.) Jeswiet, Arch. Suikerindustr. Ned. Ned. - Inde 12 399. 1925.

Habit: Grass.

Specimens examined: 20.xi.1964, Nishangara range, G. Panigrahi \& O.P. Mishra 6437 (BSA); 10.ii.1965, Nishangara range, O.P. Mishra 7915 (BSA); 08.xii.1986,
K.K. Khanna \& R. Saran 37656 (BSA); 09.xi.2002, road side, Katerniaghat range, coll. S.D. Maliya 224553 (LWG).

Saccharum narenga (Nees ex Steud.) Hack. in DC., Monogr. Phan. 6: 119. 1889.

Eriochrysis narenga Nees ex Steud., Syn. PI. Glumac. 1: 411. 1854. Eriochrysis porphyrocoma Hance, J. Bot. 14: 294. 1876. Narenga porphyrocoma (Hance) Bor, Indian Forester 66: 268. 1940; Panigrahi et al., Bull. Bot. Surv. India 11 (1 \& 2): 113. 1969.

Habit: Grass.

Specimens examined: 20.xi.1964, Nishangara range, G. Panigrahi \& O.P. Mishra 6436 (BSA).

Saccharum spontaneum L., Mant. PI. 2: 183. 1771; Hook. f. in Hook. f., Fl. Brit. India7: 118. 1897; Panigrahi et al., Bull. Bot. Surv. India 11 (1 \& 2): 113. 1969; Saini, J. Econ. Taxon. Bot. 29 (4): 916. 2005; Maliya \& Datt, J. Econ. Taxon. Bot. 34 (1): 67. 2010.

Habit: Grass.

Specimens examined: 20.xi.1964, Nishangara, O.P. Mishra 6467 (BSA); 28.xii.2003, Kuthiyaghat forest, coll. S.D. Maliya 225282 (LWG); 24.x.2004, road side, Kailashpuri forest, coll. S.D. Maliya 227104 (LWG).

Sacciolepis myosuroides (R. Br.) Camus in Lecomte, Fl. Indo-Chine 7: 460. 1922; Panigrahi et al., Bull. Bot. Surv. India 11 (1 \& 2): 113. 1969.

Panicum myosuroides R. Br., Prodr. Fl. Nov. Holl. 189. 1810.

Habit: Grass.

Specimens examined: 20.xi.1964, Nishangara range, G. Panigrahi \& O.P. Mishra 6460 (BSA).

**Schizachyrium brevifolium (Sw.) Nees ex Buse in Miq., PI. Jungh. 359. 1854; Panigrahi et al., Bull. Bot. Surv. India 11 (1 \& 2): 113. 1969; Saini, J. Econ. Taxon. Bot. 29 (4): 917. 2005.

Andropogon brevifolius Sw., Prodr. Veg. Ind. Occ. 26. 1788; Hook. f. in Hook. f., Fl. Brit. India 7: 165. 1897.

Habit: Grass.

Specimens examined: 24.xi.1964, Nishangara range, G. Panigrahi \& O.P. Mishra 6608 (BSA).

**Setaria pumila (Poir.) Roem. \& Schult., Syst. Veg. 2: 891. 1817; Maliya \& Datt, J. Econ. Taxon. Bot. 34 (1): 68. 2010.

Panicum pumilum Poir. in Lam., Encycl., Suppl. 4: 273. 1816. P. pallidifuscum Schumach., Beskr. Guin. PI. 58. 1827. Setaria pallidifusca (Schumach.) Stapf \& Hubb., Bull. Misc. Inform. Kew 1930: 259. 1930; Panigrahi et al., 
Bull. Bot. Surv. India 11 (1 \& 2): 113. 1969; Saini, J. Econ. Taxon. Bot. 29 (4): 917. 2005.

Habit: Grass.

Specimens examined: 20.xi.1964, Nishangara range, G. Panigrahi \& O.P. Mishra 6486 (BSA); 24.xi.1964, Nishangara range, G. Panigrahi \& O.P. Mishra 6594 (BSA); 14.ii.1965, Urai tal, Bichhiya, O.P. Mishra 7981 (BSA); 25.viii.2002, Murtiha range, coll. S.D. Maliya 223695 (LWG).

**Setaria verticillata (L.) P. Beauv., Ess. Agrostogr. 51, 171, 178. 1812; Hook. f. in Hook. f., Fl. Brit. India 7: 80. 1897; Maliya \& Datt, J. Econ. Taxon. Bot. 34 (1): 67.2010.

Panicum verticillatum L., Sp. PI. 82. 1762.

Habit: Grass.

Specimens examined: 23.viii.2002, Nishangara range, coll. S.D. Maliya 223621 (LWG).

Sporobolus coromandelianus (Retz.) Kunth, Revis. Gramin. 1: 68. 1829; Maliya \& Datt, J. Econ. Taxon. Bot. 34 (1): 68. 2010.

Agrostis coromandeliana Retz., Observ. Bot. 4: 19. 1786.

Habit: Grass.

Specimens examined: 25.viii.2002, Murtiha range, coll. S.D. Maliya 223703 (LWG).

Sporobolus diandrus (Retz.) Beauv., Ess. Agrostogr. 26. 1812 ( not 'diander'); Hook. f. in Hook. f., Fl. Brit. India 7: 247. 1897; Saini, J. Econ. Taxon. Bot. 29 (4): 918. 2005; Maliya \& Datt, J. Econ. Taxon. Bot. 34 (1): 68. 2010.

Agrostis diandra Retz., Observ. Bot. 5: 19. 1789.

Habit: Grass.

Specimens examined: 25.viii.2002, Murtiha range, coll. S.D. Maliya 223704 (LWG).

**Triticum aestivum L., Sp. PI. 85. 1753; Maliya \& Datt, J. Econ. Taxon. Bot. 34 (1): 68. 2010.

Habit: Grass.

Specimens examined: 20.iii.2004, Girijapuri forest, coll. S.D. Maliya 225926 (LWG).

\section{PONTEDERIACEAE}

**Eichhornia crassipes (Mart.) Solms in DC., Monogr. Phan. 4: 527. 1883; Raizada, Suppl. Fl. Upp. Gang. Plain 279. 1976; Maliya \& Datt, J. Econ. Taxon. Bot. 34 (1): 65. 2010.

Pontederia crassipes Mart., Nov. Gen. Sp. PI. 1: 9, t. 4. 1823.

Habit: Aquatic herb.

Specimens examined: 22.x.2004, Kuthiyaghat forest, coll. S.D. Maliya 225994 (LWG).

Monochoria hastata (L.) Solms in DC., Monogr. Phan. 4: 523. 1883; Panigrahi et al., Bull. Bot. Surv. India 11 (1 \& 2): 110.1969.

Pontenderia hastata L., Sp. PI. 288. 1753.

Habit: Perennial herb.

Specimens examined: 22.xi.1964, Motipur on the way to Orla, G. Panigrahi \& O.P. Mishra 6550 (BSA).

Monochoria vaginalis (Burm. f.) Presl., Reliq. Haenk. 1: 128. 1827; Hook. f. in Hook. f., Fl. Brit. India 6: 363. 1892; Duthie, Fl. Upp. Gang. Plain 3: 336. repr. ed. 1960; Saini, J. Econ. Taxon. Bot. 29 (4): 884. 2005; Maliya \& Datt, J. Econ. Taxon. Bot. 34 (1): 65. 2010.

Pontederia vaginalis Burm. f., Fl. Ind. 80. 1768.

Habit: Herb.

Specimens examined: 30.viii.2003, rest house, Murtiha range, coll. S.D. Maliya 214868 (LWG);

\section{Potamogetonaceae}

**Potamogeton crispus L., Sp. PI. 126. 1753; Hook. f. in Hook. f., Fl. Brit. India 6: 566. 1893; Duthie, Fl. Upp. Gang. Plain 3: 375. repr. ed. 1960; Panigrahi et al., Bull. Bot. Surv. India 11 (1 \& 2): 109. 1969.

Habit: Aquatic herb.

Specimens examined: 16.x.1965, Katerniaghat range, O.P. Mishra 8011 (BSA).

**Potamogeton nodosus Poir. in Lam., Encycl., Suppl. 4 (2): 535. 1816; Panigrahi et al., Bull. Bot. Surv. India 11 (1 \& 2): 109. 1969.

P. indicus Roxb., Fl. Ind. 1: 452. 1820; Hook. f. in Hook. f., FI. Brit. India 6: 565. 1893; Duthie, Fl. Upp. Gang. Plain 3: 374. repr. ed. 1960.

Habit: Aquatic herb.

Specimens examined: 11.iii.1964, Motipur range, G. Panigrahi 2825 (BSA).

\section{SMILACACEAE}

Smilax ovalifolia Roxb. ex D. Don, Prodr. Fl. Nepal. 49. 1825; Maliya \& Datt, J. Econ. Taxon. Bot. 34 (1): 65. 2010.

S. macrophylla Roxb., Fl. Ind. 3: 793. 1832; Hook. f. in Hook. f., Fl. Brit. India 6: 310. 1892; Duthie, Fl. Upp. Gang. Plain 3: 329. repr. ed. 1960 (non Willd. 1806).

Habit: Climber.

Specimens examined: 25.xi.1987, Nishangara range, K.K. Singh 6188 (LWG); 19.vii.2004, Kuthiyaghat forest, coll. S.D. Maliya 225965 (LWG); 19.xii.2004, Kuthiyaghat forest, coll. S.D. Maliya 225962 (LWG); 29.i.2005, rest house, Nishangara range, coll. S.D. Maliya 224691 (LWG) 
(Image 61).

Smilax zeylanica L., Sp. PI. 1029. 1753; Hook. f. in Hook. f., Fl. Brit. India 6: 309. 1892; Saini, J. Econ. Taxon. Bot. 29 (4): 884. 2005.

Habit: Climber.

Specimens examined: Not observed. Record based on Saini (2005).

\section{TYPHACEAE}

**Sparganium erectum L., Sp. PI. 971. 1753.

S. ramosum Huds., Fl. Angl. 2: 401. 1778, nom. illg.; Duthie, Fl. Upp. Gang. Plain 3: 359. repr. ed. 1960; Panigrahi et al., Bull. Bot. Surv. India 11 (1 \& 2): 110. 1969. Habit: Aquatic herb.

Specimens examined: 12.iii.1964, Motipur range, Katerniaghat Wildlife Sanctuary, Bharaich, G. Panigrahi 2847 (BSA).

**Typha angustifolia L., Sp. PI. 971. 1753; Maliya \& Datt, J. Econ. Taxon. Bot. 34 (1): 65. 2010.

Habit: Aquatic herb.

Specimens examined: 27.viii.2003, Katerniaghat range, Katerniaghat wildlife sanctary, Bahraich, coll. S.D. Maliya 214841 (LWG); 19.vii.2004, Kuthiyaghat forest, Katerniaghat wildlife sanctary, Bahraich, coll. S.D. Maliya 225966 (LWG); 21.iv.2010, near Gaura village, Kakraha range, L.B. Chaudhary, A. Kumar \& O. Bajpai 252229 (LWG).

\section{XANTHORRHOEACEAE}

Asphodelus tenuifolius Cav., Anales Ci. Nat. 3: 46, t. 27, f. 2. 1801; Hook. f. in Hook. f., Fl. Brit. India 6: 332. 1892; Duthie, Fl. Upp. Gang. Plain 3: 333. repr. ed. 1960; Saini, J. Econ. Taxon. Bot. 29 (4): 884. 2005; Maliya \& Datt, J. Econ. Taxon. Bot. 34 (1): 65. 2010.

Habit: Herb.

Specimens examined: 21.xii.2003, Katerniaghat range, Katerniaghat wildlife sanctary, Bahraich, coll. S.D. Maliya 225227 (LWG).

\section{ZINGIBERACEAE}

**Amomum maximum Roxb., Asiat. Res. 11: 344. 1810.

A. dealbatum Roxb., Fl. Ind. (ed. Carey \& Wall) 1: 42. 1820; Panigrahi et al., Bull. Bot. Surv. India 11 (1 \& 2): 109.1969.

Habit: Herb.

Specimens examined: 20.xi.1964, Nishangara range, G. Panigrahi \& O.P. Mishra 6480 (BSA).

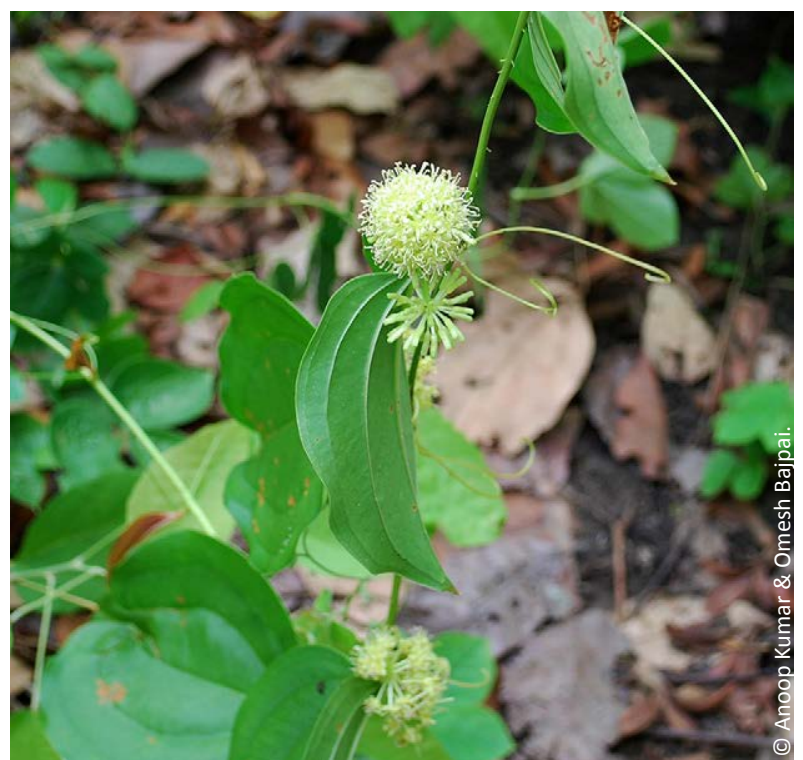

Image 61. Smilax ovalifolia Roxb. ex D. Don

Cheilocostus speciosus (Koenig.) Specht, Taxon 55: 159. 2006.

Bankisa speciosa Koenig in Retz., Observ. Bot. 3: 75. 1783. Costus specious (Koenig) Sm., Trans. Linn. Soc. London 1: 249. 1791; Baker in Hook. f., Fl. Brit. India 6: 249. 1892; Duthie, Fl. Upp. Gang. Plain 3: 310. repr. ed. 1960; Saini, J. Econ. Taxon. Bot. 29 (4): 882. 2005.

Habit: Herb.

Specimens examined: Not observed. Record based on Saini (2005).

Curcuma aromatica Salisb., Parad. London 1 (2): t. 96. 1807; Maliya \& Datt, J. Econ. Taxon. Bot. 34 (1): 64.2010. Habit: Herb.

Specimens examined: 27.viii.2003, road side, Katerniaghat range, coll. S.D. Maliya 214846 (LWG); 16.vii.2004, Kuthiyaghat forest, coll. S.D. Maliya 225950 (LWG); 05.ix.2005, Girijapuri forest, coll. S.D. Maliya 227149 (LWG).

*Curcuma longa L., Sp. PI. 2. 1753; Duthie, Fl. Upp. Gang. Plain 3: 309. repr. ed. 1960; Maliya \& Datt, J. Econ. Taxon. Bot. 34 (1): 64. 2010.

C. domestica Val., Bull. Jard. Bot. Buitenzorj Ser. 2, 27: 31. 1918.

Habit: Herb, cultivated.

Specimens examined: 31.viii.2003, Bishunpura village, coll. S.D. Maliya 214886 (LWG); 27.xii.2005, Lahaura village, Nishangara range, coll. S.D. Maliya 227192 (LWG). 
Hedychium coronarium Koenig in Retz. Observ. Bot. 3: 73. 1783; Duthie, Fl. Upp. Gang. Plain 3: 307. repr. ed. 1960; Panigrahi et al., Bull. Bot. Surv. India 11 (1 \& 2): 109. 1969.

Habit: Herb.

Specimens examined: Not observed. Record based on Panigrahi et al. (1969).

Zingiber roseum (Roxb.) Roscoe, Trans. Linn. Soc. London 8: 348. 1807.

Amomum roseum Roxb., Pl. Coromandel, 2: 15, pl. 126. 1800.

Habit: Herb.

Specimens examined: 09.xi.2002, rest house, Katerniaghat range, coll. S.D. Maliya 224533 (LWG); 24.xii.2003, Kuthiyaghat forest, coll. S.D. Maliya 225247 (LWG); 24.x.2004, Girijapuri forest, coll. S.D. Maliya 227106 (LWG).

* - Cultivated species and ${ }^{* *}$ - Alien invasive species

\section{REFERENCES}

Anonymous (2011). Forest Survey of India. (http://www.fsi.org.in/fsi projects/annualreport.pdf).

Bajpai, O., A. Kumar, A.K. Mishra, N. Sahu, S.K. Behera \& L.B. Chaudhary (2012). Phenological study of two dominant tree species in tropica moist deciduous forest from the Northern India. International Journal of Botany 8(2): 66-72.

Behera, S.K., A.K. Mishra, N. Sahu, A. Kumar, N. Singh, A. Kumar, O. Bajpai, L.B. Chaudhary, P.B. Khare \& R. Tuli (2012). The study of microclimate in response to different plant community associationin tropical moist deciduous forest from northern India. Biodiversity and Conservation 21(5): 1159-1176; http://dx.doi.org/10.1007/s10531012-0230-5
Jain, S.K. \& R.R. Rao (1977). A Handbook of Field and Herbarium Methods. Today \& Tomorrow's Printers \& Publishers, New Delhi.

Kumar, A., O. Bajpai, R. Tiwari, J.V. Sudhakar \& L.B. Chaudhary (2012). Taxonomic notes on the identity and nomenclature of Ficus assamica Miq. (Moraceae). Phytotaxonomy 12: 82-88.

Lawrence, G.H.M. (1951). Taxonomy of Vascular Plants. Oxford IBH Publishing Co. Pvt. Ltd. New Delhi.

Maliya, S.D. (2011). New or less known uses of some ethnomedicinal plants of Wildlife Sanctuary Katarniyaghat of Bahraich Uttar Pradesh. Journal of Economic and Taxonomic Botany 35(1): 35-38.

Maliya, S.D. (2012). Aquatic and wetland macrophytes of Katarniyaghat Wildlife Sanctuary of Bahraich District, Uttar Pradesh, (India). Journal of Economic and Taxonomic Botany 35(1): 156-165.

Maliya, S.D. (2012a). Additions to the flora of Katarniyaghat Wildlife Sanctuary, Bahraich district, Uttar Pradesh. Journal of Economic and Taxonomic Botany 36(2): 419-426.

Maliya, S.D. \& B. Datt (2010). A contribution to the flora of Katarniyaghat Wildlife Sanctuary, Bahraich District, Uttar Pradesh. Journal of Economic and Taxonomic Botany 34(1): 42-68.

Pandey, R., T.P. Mall \& R.K. Singh (2008). Forest types of Katerniaghat Wildlife Sanctuary - A biogeographic classification. Environmental Conservation Journal 9(3): 83-87.

Pandey, R. \& Mall, T. P. (2009). Ethnobotanical plants of Katerniaghat Wildlife Sanctuary. Vegetos 22(1): 135-138.

Pandey, R., T.P. Mall \& R.K. Singh (2009). Phytodiversity of Katerniaghat Wildlife Sanctuary. Environmental Conservation Journal 10(1\&2): 77-79.

Panigrahi, G., A.N. Singh \& O.P. Mishra (1969). Contribution to the botany of the terai forest of the Bahraich District of Uttar Pradesh. Bulletin of the Botanical Survery of India 11(1\&2): 89-114.

Saini, D.C. (2005). Flora of Bahraich District, Uttar Pradesh - I-IV. Journal of Economic and Taxonomic Botany 29(3): 528-636.

Saini, D.C. (2005). Flora of Bahraich District, Uttar Pradesh - V-VI. Journal of Economic and Taxonomic Botany 29(4): 843-920.

Srivastava, S.K. (2004). Floristic diversity in Uttar Pradesh- an overview. Journal of Economic and Taxonomic Botany 28(2): 292-334.

Srivastava, S.K. (2011). Plant diversity and conservation strategies of Uttar Pradesh. Phytotaxonomy 11: 45-62.

Tripathi, K.P. \& B. Singh (2009). Species diversity and vegetation structure across various strata in natural and plantation forests in Katerniaghat Wildlife Sanctuary, north India. Tropical Ecology 50(1): 191-200.

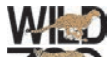

